\title{
Selected Papers from Building A Better New Zealand (BBNZ 2014) Conference
}

Edited by

George Baird, Lois Easton and

Adrian Bennett

Printed Edition of the Special Issue Published in Buildings 
George Baird, Lois Easton and Adrian Bennett (Eds.)

\section{Selected Papers from Building A Better New Zealand (BBNZ 2014) Conference}


This book is a reprint of the Special Issue that appeared in the online, open access journal, Buildings (ISSN 2075-5309) in 2015 (available at:

http://www.mdpi.com/journal/buildings/special_issues/bbnz2014).

\section{Guest Editors}

George Baird

School of Architecture, Victoria University of Wellington

New Zealand

Lois Easton

Beacon Pathway, Auckland

New Zealand

Adrian Bennett

Building System Performance, Ministry of Business

Innovation \& Employment

New Zealand

Editorial Office

MDPI AG

Klybeckstrasse 64

Basel, Switzerland
Publisher

Shu-Kun Lin
Editorial Manager

Alistair Freeland

\section{Edition 2016}

MDPI • Basel $\bullet$ Beijing $\bullet$ Wuhan $\bullet$ Barcelona

ISBN 978-3-03842-095-8 (PDF)

ISBN 978-3-03842-096-5 (Hbk)

(C) 2016 by the authors; licensee MDPI, Basel, Switzerland. All articles in this volume are Open Access distributed under the Creative Commons Attribution license (CC BY), which allows users to download, copy and build upon published articles even for commercial purposes, as long as the author and publisher are properly credited, which ensures maximum dissemination and a wider impact of our publications. However, the dissemination and distribution of copies of this book as a whole is restricted to MDPI, Basel, Switzerland. 


\section{Table of Contents}

List of Contributors VII

About the Guest Editors IX

Introduction and Editorial to the Special Issue "Selected Papers from the Building a Better New Zealand (BBNZ 2014) Conference" XI

\section{Part I: Achieving Better Buildings}

Mikael Boulic, Robyn Anne Phipps, Malcolm Cunningham, Don John Cleland, Pär Fjällström, Keiko Abe and Philippa Howden-Chapman

Heater Choice, Dampness and Mould Growth in 26 New Zealand Homes: A Study of Propensity for Mould Growth Using Encapsulated Fungal Spores

Reprinted from: Buildings 2015, 5(1), 149-162

http://www.mdpi.com/2075-5309/5/1/149 3

\section{Roger Birchmore, Andy Pivac and Robert Tait}

Impacts of an Innovative Residential Construction Method on Internal Conditions

Reprinted from: Buildings 2015, 5(1), 179-195

http://www.mdpi.com/2075-5309/5/1/179 17

\section{Paola Leardini and Manfredo Manfredini}

Modern Housing Retrofit: Assessment of Upgrade Packages to EnerPHit Standard for 1940-1960 State Houses in Auckland

Reprinted from: Buildings 2015, 5(1), 229-251

http://www.mdpi.com/2075-5309/5/1/229 .35

\section{Mark Bassett and Greg Overton}

Measuring the Weathertight Performance of Flashings

Reprinted from: Buildings 2015, 5(1), 130-148

http://www.mdpi.com/2075-5309/5/1/130 


\section{Shaan Cory, Michael Donn and Andrew Pollard}

Comparison of NZ's Energy Efficiency Regulation and Verification Assumptions to Real Building Loads and Operation

Reprinted from: Buildings 2015, 5(1), 116-129

http://www.mdpi.com/2075-5309/5/1/116 78

\section{Part II: Productivity}

\section{Wajiha Shahzad, Jasper Mbachu and Niluka Domingo}

Marginal Productivity Gained Through Prefabrication: Case Studies of Building Projects in Auckland

Reprinted from: Buildings 2015, 5(1), 196-208

http://www.mdpi.com/2075-5309/5/1/196 .95

\section{Jianchao Zhang, Boon-Chong Seet and Tek Tjing Lie}

Building Information Modelling for Smart Built Environments

Reprinted from: Buildings 2015, 5(1), 100-115

http://www.mdpi.com/2075-5309/5/1/100 108

\section{Mohammadali Noktehdan, Mehdi Shahbazpour and Suzanne Wilkinson}

Driving Innovative Thinking in the New Zealand Construction Industry

Reprinted from: Buildings 2015, 5(2), 297-309

http://www.mdpi.com/2075-5309/5/2/297

\section{Ricardo Antunes and Vicente Gonzalez}

A Production Model for Construction: A Theoretical Framework

Reprinted from: Buildings 2015, 5(1), 209-228

http://www.mdpi.com/2075-5309/5/1/209

\section{Part III: Urban Issues}

\section{Natalie Allen}

Understanding the Importance of Urban Amenities: A Case Study from Auckland Reprinted from: Buildings 2015, 5(1), 85-99 


\section{Morten Gjerde and Brenda Vale}

Aiming for a Better Public Realm: Gauging the Effectiveness of Design Control Methods in Wellington, New Zealand

Reprinted from: Buildings 2015, 5(1), 69-84

http://www.mdpi.com/2075-5309/5/1/69 176

\section{A. Santos Nouri}

A Framework of Thermal Sensitive Urban Design Benchmarks: Potentiating the Longevity of Auckland's Public Realm

Reprinted from: Buildings 2015, 5, 252-281

http://www.mdpi.com/2075-5309/5/1/252 



\section{List of Contributors}

Keiko Abe: Institute of Environmental Biology, JDC Corporation, Kanagawa 243-03, Japan Natalie Allen: School of Architecture and Planning, The University of Auckland, 26 Symonds St, Auckland 1142, New Zealand

Ricardo Antunes: Department of Civil and Environmental Engineering, University of Auckland, 20 Symonds Street, Auckland 1142, New Zealand

George Baird: School of Architecture, Victoria University of Wellington, PO Box 600, Wellington 6140, New Zealand

Mark Bassett: BRANZ Ltd., P.O. Box 50908, Porirua City 5240, New Zealand

Roger Birchmore: Department of Construction, Unitec Institute of Technology, P.O. Box 92025 Auckland, New Zealand

Mikael Boulic: School of Engineering and Advanced Technology, Massey University, Manawatu Campus, Palmerston North 4474, New Zealand

Don John Cleland: School of Engineering and Advanced Technology, Massey University, Manawatu Campus, Palmerston North 4474, New Zealand

Shaan Cory: Centre for Building Performance Research, Victoria University of Wellington, P.O. Box 600, Wellington 6140, New Zealand

Malcolm Cunningham: Building Research Association of New Zealand, Porirua 5381, New Zealand

Niluka Domingo: School of Engineering and Advanced Technology, Massey University, P/Bag 102904, Auckland 0745, New Zealand

Michael Donn: Centre for Building Performance Research, Victoria University of Wellington, P.O. Box 600, Wellington 6140, New Zealand

Pär Fjällström: IVL Swedish Environmental Research Institute, P.O. Box 210 60, SE-100 31 Stockholm, Sweden

Morten Gjerde: School of Architecture, Victoria University of Wellington, P.O. Box 600, Wellington 6140, New Zealand

Vicente Gonzalez: Department of Civil and Environmental Engineering, University of Auckland, 20 Symonds Street, Auckland 1142, New Zealand

Philippa Howden-Chapman: Department of Public Health, University of Otago, Wellington 6021, New Zealand

Paola Leardini: School of Architecture and Planning, The University of Auckland, 26 Symonds Street, 1010 Auckland, New Zealand

Tek Tjing Lie: Department of Electrical and Electronic Engineering, Auckland University of Technology, Auckland 1010, New Zealand

Manfredo Manfredini: School of Architecture and Planning, The University of Auckland, 26 Symonds Street, 1010 Auckland, New Zealand

Jasper Mbachu: School of Engineering and Advanced Technology, Massey University, P/Bag 102904, Auckland 0745, New Zealand 
Mohammadali Noktehdan: Department of Civil and Environmental Engineering, The University of Auckland, Private Bag 92019,Auckland 1142, New Zealand

A. Santos Nouri: CIAUD, Faculty of Architecture, University of Lisbon, Rua Sa Nogueira, Polo Universitario, Alto da Ajuda, 1349-055 Lisbon, Portugal

Greg Overton: BRANZ Ltd., P.O. Box 50908, Porirua City 5240, New Zealand

Robyn Anne Phipps: School of Engineering and Advanced Technology, Massey University, Albany Campus, Auckland 0632, New Zealand

Andy Pivac: Department of Building Technology, Unitec Institute of Technology, P.O. Box 92025 Auckland, New Zealand

Andrew Pollard: Building Research Association of New Zealand (BRANZ) Ltd, Private Bag 50908, Porirua 5240, New Zealand

Boon-Chong Seet: Department of Electrical and Electronic Engineering, Auckland University of Technology, Auckland 1010, New Zealand

Mehdi Shahbazpour: Department of Mechanical Engineering, The University of Auckland, Private Bag 92019, Auckland 1142, New Zealand

Wajiha Shahzad: School of Engineering and Advanced Technology, Massey University, P/Bag 102904, Auckland 0745, New Zealand

Robert Tait: Department of Building Technology, Unitec Institute of Technology, P.O. Box 92025 Auckland, New Zealand

Brenda Vale: School of Architecture, Victoria University of Wellington, P.O. Box 600, Wellington 6140, New Zealand

Suzanne Wilkinson: Department of Civil and Environmental Engineering, The University of Auckland, Private Bag 92019,Auckland 1142, New Zealand

Jianchao Zhang: Department of Electrical and Electronic Engineering, Auckland University of Technology, Auckland 1010, New Zealand 


\section{About the Guest Editors}

George Baird is an Emeritus Professor of Building Science at the School of Architecture, Victoria University of Wellington, New Zealand, where he specializes in building environmental science and sustainable engineering systems design, building performance generally, and the energy efficient design and operation of buildings.

Dr. Baird is a Fellow of the Chartered Institution of Building Services Engineers (UK) and of the Institution of Professional Engineers New Zealand, and a Life Member of the Energy Management Association of New Zealand.

He received the 1999 NZ Science and Technology Bronze Medal "for his singular contribution to energy efficiency of New Zealand buildings and to building performance research" and the 2006 international "Pioneers of the World Renewable Energy Network" Award for his "contribution to the world of renewable energy".

Dr. Baird is the author of innumerable technical papers. His major books include: Energy Performance of Buildings, Building Evaluation Techniques, Architectural Expression of Environmental Control Systems, and most recently, Sustainable Buildings in Practice, a worldwide survey of sustainable buildings.

For further details see:

http://www.victoria.ac.nz/architecture/staff/george-baird.aspx

Adrian Bennett is the Principal Advisor, Building Science, at the Ministry of Business, Innovation and Employment (MBIE) in New Zealand. One of MBIE's responsibilities is the building regulatory system including the Building Act and the national building code. Adrian works in the Engineering Design and Science Team and is responsible for commissioning research to support the development and maintenance of the building code and supporting documents. In this role, he works closely with research funders and researchers in universities and research organisations.

$\mathrm{He}$ has previously managed a materials science research team at the Building Research Association of New Zealand.

Lois Easton is an environmental scientist and researcher who has worked extensively in local government, and as an environmental consultant providing advice to a variety of government and not for profit organisations.

For many years the Sustainable Homes Research Team Leader for Beacon Pathway Inc., a sustainable housing research consortium, Lois led a range of sustainable new housing and retrofit research and demonstration projects, as well as investigating policy to support sustainable building initiatives. Lois also has held a number of senior roles at Waitakere City Council where she was responsible for a number of sustainable building projects. Lois has also lectured in Sustainable Development at Auckland University's Department of Architecture and Planning. 



\section{Introduction and Editorial to the Special Issue "Selected Papers from the Building a Better New Zealand (BBNZ 2014) Conference"}

\section{George Baird}

Reprinted from Buildings. Cite as: Baird, G. Introduction and Editorial to the Special Issue "Selected Papers from the Building a Better New Zealand (BBNZ 2014) Conference". Buildings 2015, 5, 318-322.

\section{The Background to the Conference}

The current "Building a Better New Zealand" initiative began in 2009 with the New Zealand Government and the Building Industry working together to develop "Building the Future" - a document that looked at the challenges and opportunities facing the country's building and construction industry in coming decades.

That document made the need for an "overarching building and construction research strategy" very clear. It was recognised that a successful and innovative building industry would be critical if the country was to provide the homes, workplaces and communities that would secure firm foundations for its future. The country also faced the distinct challenges and opportunities of rebuilding the city of Christchurch, following the devastating earthquake of 2011; and coping with a projected $40 \%$ increase in the population of its largest city, Auckland, over the next 25 years.

Thus it was, following extensive consultation with government and industry during 2012, that a Building a Better New Zealand Research Strategy Document was developed jointly by the Building Research Association of New Zealand, the Construction Industry Council, and the Ministry of Business Innovation and Employment. Its publication in June 2013 provided the incentive and inspiration for the organising committee of what became known as the Building a Better New Zealand Conference (BBNZ 2014) that was held in Auckland from 3 to 5 September 2014 and was planned around the following themes:

- Achieving better buildings;

- Improving the performance of materials;

- Improving the performance of existing buildings;

- Sustainability;

- Productivity;

- Meeting New Zealanders housing needs;

- Building better cities and communities.

Deliberately geared to provide a forum for researchers and industry, the conference accepted some 55 academic papers and around 45 industry presentations, covering the seven main themes, as well as a number from keynote speakers. These had been subjected to blind peer reviewing by the Conference Scientific Committee chaired by Professor George Baird, thus ensuring high quality papers and presentations. Full details of the Research Strategy Document, the Industry Needs 
Survey that preceded it, and the Building a Better New Zealand Conference itself may be found at the following website: http://www.buildingabetternewzealand.co.nz.

As a member of the Editorial Board of Buildings, Professor Baird explored the possibility of having a Special Issue of the journal, made up of around ten Selected Papers. His proposal was readily accepted and the authors of the academic papers most highly ranked in the conference peer review process were invited to develop their papers further and submit them to the Journal's rigorous review process.

In the event, twelve papers were accepted and form the content of this Special Issue and while the original conference papers tended to have a New Zealand focus, most authors have looked at the international applicability of their findings in these revised and expanded journal articles if they had not already done so.

\section{Content of the Special Issue}

While the selected papers were nominally spread across all of the conference themes, with "Achieving Better Buildings" and "Productivity" the most popular, in practice most papers covered more than one theme. For the purposes of this editorial they are grouped under these two headings and a third I have labelled "Urban Issues".

\subsection{Achieving Better Buildings}

Papers by Boulic et al. [1], Birchmore et al. [2], Leardini and Manfredini [3], Bassett and Overton [4], and Cory et al. [5] make up this group. Their predominant focus is on achieving better conditions in domestic buildings.

As noted by Leardini and Manfredini of the School of Architecture and Planning at Auckland University, while much of New Zealand's housing stock is soundly constructed, many homes are uninsulated, with all the attendant problems that follow. They describe the results of a study of different retrofit upgrade packages to a section of the Auckland housing stock, concluding that "nearly zero-energy buildings .... can be achieved through retrofitting interventions that meet the EnerPHit standard" [3].

Birchmore, Pivac, and Tait from the Departments of Construction and of Building Technology at Auckland's Unitec Institute of Technology carried out full scale testing of an innovative construction method. This involved the use of a vapour check and a plywood barrier, and resulted in improved thermal resistance, reduced infiltration, and less risk of interstitial condensation [2].

Boulic et al. [1] evaluated mould growth in the living rooms and bedrooms of two sets of occupied houses, one set with unflued gas heaters, the other with electric heat pumps. Perhaps unsurprisingly, the unflued gas heaters were found to be a significant additional source of moisture, and the houses in which they were fitted had average daily hyphal growth rates several times higher than the houses fitted with the heat pumps.

Bassett and Overton of the Building Research Association of New Zealand investigated the performance of three different forms of flashing under dynamic wind and rain loads. All three 
were found to resist water leakage, but the influence of vents and other air leakage paths remains to be studied [4].

The final paper in this group, by Cory, Donn, and Pollard of the Centre for Building Performance Research at Victoria University of Wellington's School of Architecture and the Building Research Association of New Zealand aims to make the energy modelling of commercial buildings much more realistic. Using data from a national building energy end-use study, the authors present typical, high, and low energy load densities and their operational patterns for commercial buildings [5].

\subsection{Productivity}

These four papers by Shahzad et al. [6]; Zhang et al. [7], Noktehdan et al. [8] and Antunes and Gonzales [9], all deal with matters related to construction industry productivity.

Shahzad, Mbachu and Domingo from the School of Engineering and Advanced Technology at Massey University in Auckland analysed 66 Auckland building projects where prefabrication had been employed to assess productivity differences from traditional building systems. They found that prefabrication resulted in average reductions in completion times and costs of $34 \%$ and $19 \%$ respectively. This translated to an overall average productivity improvement of $7 \%$, with housing the most improved at $11 \%$ [6].

In their contribution, Noktehdan, Shahbazpour and Wilkinson from the Departments of Civil and Environmental Engineering and of Mechanical Engineering at the University of Auckland examine the relationship between innovation and productivity improvement in the construction industry. Having developed an innovation classification system they go on to demonstrate it via a database of 500 innovations, and proposing its use for calculating innovation performance indicators [8].

Antunes and Gonzales, also from the School of Civil and Environmental Engineering at the University of Auckland, set out to examine existing knowledge about production models in order to establish a foundation for dynamic production systems management in construction, proposing a theoretical framework for the future development of mathematical production models aimed at predicting performance [9].

No discussion of productivity would be complete without the topic of Building Information Modelling (BIM). In their paper, Zhang, Seet and Lie from the Department of Electrical and Electronic Engineering at Auckland University of Technology investigate how BIM can contribute to the development of Smart Built Environments from the design phase to facility management [7].

\subsection{Urban Issues}

Papers by Allen [10], Gjerde and Vale [11] and Nouri [12] deal with a variety of important urban issues.

Allen from the School of Architecture and Planning at the University of Auckland is concerned with the role that urban amenities play in providing a sense of liveability for the residents of a neighbourhood. Based on the results of 57 face-to-face interviews in four Auckland suburbs, 
insights were gained into the trade-offs residents were prepared to make when choosing to live in medium-density housing typologies [10].

Gjerde and Vale from the School of Architecture at Victoria University of Wellington investigated the effectiveness of design review systems in ensuring the visual quality of streetscapes. Results of a case study of a Wellington street are presented and the authors speculate how such systems could be made to work more effectively [11].

Additionally, concerned with the urban realm, Nouri from the Faculty of Architecture at the University of Lisbon addresses the issue of thermal comfort in public spaces. Following an extensive international review the author develops a framework of relevant bioclimatic case studies, arguing that there is sufficient existing knowledge to respond to the growing need for thermal comfort attenuation in the city of Auckland [12].

\section{Acknowledgments}

Finally, it is my pleasure to thank my Co-Guest Editors Lois Easton and Adrian Bennett for their assistance, the reviewers for their exacting commentaries and suggestions, all the authors for responding to these with alacrity, and of course the team at MDPI for facilitating this Special Issue so efficiently.

As a last word, it is gratifying to note that four of the selected papers were from $\mathrm{PhD}$ candidates, all of whom received a student award at the Conference - auguring well for future research towards the building of a better New Zealand.

\section{Conflicts of Interest}

The author declares no conflict of interest.

\section{References}

1. Boulic, M.; Phipps, R.A.; Cunningham, M.; Cleland, D.J.; Fjällström, P.; Abe, K.; Howden-Chapman, P. Heater Choice, Dampness and Mould Growth in 26 New Zealand Homes: A Study of Propensity for Mould Growth Using Encapsulated Fungal Spores. Buildings 2015, 5, 149-162.

2. Birchmore, R.; Pivac, A.; Tait, R. Impacts of an Innovative Residential Construction Method on Internal Conditions. Buildings 2015, 5, 179-195.

3. Leardini, P.; Manfredini, M. Modern Housing Retrofit: Assessment of Upgrade Packages to EnerPHit Standard for 1940-1960 State Houses in Auckland. Buildings 2015, 5, 229-251.

4. Bassett, M.; Overton, G. Measuring the Weathertight Performance of Flashings. Buildings 2015, 5, 130-148.

5. Cory, S.; Donn, M.; Pollard, A.; Comparison of NZ's Energy Efficiency Regulation and Verification Assumptions to Real Building Loads and Operation. Buildings 2015, 5, 116-129.

6. Shahzad, W.; Mbachu, J.; Domingo, N. Marginal Productivity Gained Through Prefabrication: Case Studies of Building Projects in Auckland. Buildings 2015, 5, 196-208. 
7. Zhang, J.; Seet, B.-C.; Lie, T.T. Building Information Modelling for Smart Built Environments. Buildings 2015, 5, 100-115.

8. Noktehdan, M.; Shahbazpour, M.; Wilkinson, S. Driving Innovative Thinking in the New Zealand Construction Industry. Buildings 2015, 5, 297-309.

9. Antunes, R.; Gonzalez, V. A Production Model for Construction: A Theoretical Framework. Buildings 2015, 5, 209-228.

10. Allen, N. Understanding the Importance of Urban Amenities: A Case Study from Auckland. Buildings 2015, 5, 85-99.

11. Gjerde, M.; Vale, B. Aiming for a Better Public Realm: Gauging the Effectiveness of Design Control Methods in Wellington, New Zealand. Buildings 2015, 5, 69-84.

12. Nouri, A.S. A Framework of Thermal Sensitive Urban Design Benchmarks: Potentiating the Longevity of Auckland's Public Realm. Buildings 2015, 5, 252-281. 

Part I:

Achieving Better Buildings 



\title{
Heater Choice, Dampness and Mould Growth in 26 New Zealand Homes: A Study of Propensity for Mould Growth Using Encapsulated Fungal Spores
}

\author{
Mikael Boulic, Robyn Anne Phipps, Malcolm Cunningham, Donald John Cleland, \\ Pär Fjällström, Keiko Abe and Philippa Howden-Chapman
}

\begin{abstract}
The relationship between the use of unflued gas heaters (UGH, $N=14)$ and heat pump heaters (HP, $N=12$ ) located in the living rooms, and mould growth on the living room and bedroom walls, of 26 New Zealand (NZ) occupied homes was investigated during winter. Two methods were employed to evaluate the potential of mould growth on walls: (i) measurement of daily hyphal growth rate using a fungal detector (encapsulated fungal spores); and (ii) estimation of fungal contamination based on a four level scale visual inspection. The average wall psychrometric conditions were significantly different between the two heater type groups, in both the living rooms and the bedrooms with the UGH user homes being colder and damper than HP user homes. The UGHs were found to be a significant additional source of moisture in the living rooms which dramatically increased the capacity for fungi to grow on wall surfaces. The average daily hyphal growth rates were 4 and 16 times higher in the living rooms and in the bedrooms of the UGH user homes, respectively. Results from both mould detection methods gave good agreement, showing that the use of a fungal detector was an efficient method to predict the potential of mould growth on the inside of the external walls in NZ homes.
\end{abstract}

Reprinted from Buildings. Cite as: Boulic, M.; Phipps, R.A.; Cunningham, M.; Cleland, D.J.; Fjällström, P.; Abe, K.; Howden-Chapman, P. Heater Choice, Dampness and Mould Growth in 26 New Zealand Homes: A Study of Propensity for Mould Growth Using Encapsulated Fungal Spores. Buildings 2015, 5, 149-162.

\section{Introduction}

\subsection{Background}

Houses in New Zealand (NZ) are typically damp and cold [1]. The dampness and low indoor temperature is mainly due to two factors: building insulation deficiency (houses constructed before April 1978 were not subject to regulations requiring insulation) and undersized heating systems (only around 5\% of NZ dwellings have central heating systems). In winter 2006, when the study was conducted, unflued gas heaters (UGH) were used in $27.7 \%$ of the NZ dwellings [2,3]. While UGHs use in NZ has declined since the study to $15.4 \%$ of the NZ dwellings [4], they are still in common use and hence the study is still relevant. Further, the results are useful for other countries with similar climates, housing stocks and use of UGHs. In NZ, these UGHs are mainly operated on low or medium setting which gives an estimated average heat output of $2.6 \mathrm{~kW}[5]$.

Unflued gas heaters, also called Liquefied Petroleum Gas (LPG) cabinets or portable gas heaters are designed to release combustion gases directly in the indoor environment [6]. In addition, to the 
chemical pollutants, like nitrogen dioxide [7], released during the combustion process, water vapour is another combustion byproduct which is released at a theoretical rate of $1.6 \mathrm{~kg}$ per $\mathrm{kg}$ of LPG consumed dependent on the proportion of butane and propane in the bottle. Therefore, the use of an UGH increases the room moisture level [8].

Temperature and moisture levels are critical factors for fungal development. Viable spores are always present in dwellings and germination and subsequent hyphal development can occur if the nutrients, temperature and moisture availability are suitable. The mould and their by-products could be harmful for people, particularly if they are immune deficient or asthmatic [9].

\subsection{Methods}

The Housing, Heating and Health Study (HHH Study) was a community trial to investigate the relationship between domestic heaters, indoor environment and occupant's health. Four hundred and nine families with a child with doctor diagnosed asthma (index child), and using an UGH or a portable electric heater as their main heater, were enrolled in this interventional study. Baseline health and environmental parameters were measured in all homes during the first winter/spring season. Before the second winter season, the original heater (UGH or portable electric heater) was replaced by a higher capacity and non-indoor polluting heater such as a heat pump heater (HP), for a randomly selected half of the dwellings (Treatment group). The Control group kept using their original heater during the second winter season and received their replacement heater at the end of the study. Health and environmental measurements were repeated in all homes during the second winter [10].

For a subset $(N=26)$ of the HHH Study households, who were operating either an UGH or a HP in the living rooms, the propensity for mould to grow and the indoor climate were investigated using a fungal detector (JDC Corporation, Kanagawa, Japan), and temperature/relative humidity (RH) measurements. Visual inspections of mould growth were carried out to validate the fungal detector predictions. This subset study was undertaken during winter in 26 dwellings in the Hutt Valley, a semi-coastal area that is part of Greater Wellington, New Zealand. The dwellings had been insulated (under floor and roof cavity) before the study started, according to the New Zealand Standard 4218:2004 specifications [11]. Twelve households received a HP (Treatment group) and 14 households were still using their original UGHs (Control group). The level of moisture in homes can be affected by many factors, other than unflued combustion process, like cooking, showering/bathing and drying clothes. However the households were selected with similar other moisture contributors (similar location, similar building construction and insulation level, similar level of occupancy), and it was assumed that the only difference in moisture source will be due to the operation of the heaters. The aim of this study was to investigate the moisture in homes resulting from the use of heater and to determine if a fungal detector could be a useful device to evaluate the suitability of the indoor environment for fungal development during winter time in New Zealand.

For each dwelling, the wall temperature and RH measurements were carried out in the living room and the index child's bedroom, using wall-mounted Hobo ${ }^{\circledR}$ H8 sensors (Onset Computer Corporation, Bourne, MA, USA). The loggers were attached on the interior lining of an external wall at a consistent height of $1.8 \mathrm{~m}$ above the floor level (B in Figure 1). The loggers were set to monitor 
the temperature and the RH continuously every $15 \mathrm{~min}$ for up to a 41.4 days' period (maximum memory capacity). The logger thickness placed the sensors about $1 \mathrm{~cm}$ away from the wall surface.

In close proximity to this temperature/RH sensor, a fungal detector was located directly on the wall surface (A in Figure 1). The fungal detector consisted of three inclusions of fungal spores; two xerophilic fungi (Eurotium herbariorum and Aspergillus penicilloides) and one hydrophilic fungus (Alternaria alternata) in an encapsulating device (plastic plate, water vapour permeable transparent film and double sided adhesive tape acting as a frame) [12]. Xerophilic fungi grow under relatively dry conditions and hydrophilic fungi need extremely humid conditions to grow. Each fungal inclusion contains $3 \mu \mathrm{L}$ of spore suspension at $10^{6}$ spores per $\mathrm{mL}$ concentration [13]. At the start of exposure, the spores were in a dry state. The fungal detectors were exposed to the living room and bedroom wall climate for an average period of 85 days. At the conclusion of the winter monitoring period, the three fungal inclusions (Aspergillus penicillioides, Eurotium herbariorum and Alternaria alternata) were examined under a microscope and the hyphal lengths were measured.

In addition to the fungal detector assessment method, a visual inspection of mould growth was carried out in the 26 living rooms and bedrooms by the first author. A contamination scale with four graduation levels was used to visually assess the mould level in the living room and in the child's bedroom (M0: "no visible mould", M1: "specks of visible mould", M2: "moderate visible mould patches" and M3: "extensive covered areas").

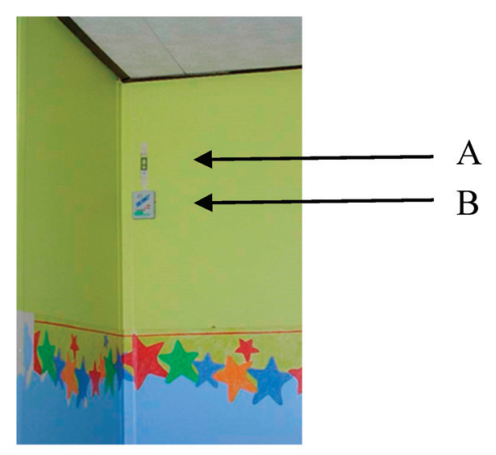

Figure 1. Typical sensor locations on the inside of the external wall in an index child's bedroom (A: fungal detector, B: temperature and relative humidity logger).

\section{Results and Discussion}

\subsection{The Wall Climate}

The temperature/RH loggers operated to full memory capacity (41.4 days). Table 1 shows the average psychrometric conditions in households operating an UGH $(N=14)$ and in households operating a $\mathrm{HP}(N=12)$.

In the living rooms, the wall climates were on average colder $\left(15.2^{\circ} \mathrm{C} v s .17 .4^{\circ} \mathrm{C}\right)$ and had higher RH $(63.6 \%$ vs. 53.5\%) in the UGH group than in the HP group. Similar results were found in the bedrooms $\left(13.9^{\circ} \mathrm{C} v s .16 .0^{\circ} \mathrm{C}\right.$ and $68.6 \%$ vs. $\left.60.9 \%\right)$ for UGH and HP groups respectively. For both 
groups, the bedrooms were colder and damper than the living rooms. In the living rooms, Table 1 shows higher humidity ratio in the UGH group than in the HP group $(6.9 \mathrm{~g} / \mathrm{kg} v s .6 .6 \mathrm{~g} / \mathrm{kg})$. This result is consistent with UGH being an additional source of moisture for the living room environment. In the bedrooms, for both groups of households, the humidity ratio was similar ( $6.8 \mathrm{~g} / \mathrm{kg} \mathrm{vs.} 6.9 \mathrm{~g} / \mathrm{kg})$. With a similar level of humidity ratio, the lower average RH for the HP user group appears to be mainly due to a higher average temperature. This result indicates that the HP seemed to be more effective than the UGH for heating more than one room in the house. The moisture released from the UGH operated in the living rooms appears to have little effect on the bedroom humidity ratio.

Households operating an UGH had a higher RH level than households operating a HP, thus the release of water vapour, during the operation of an $\mathrm{UGH}$, was found to be a significant additional source of moisture for the wall surface in the living rooms. Studies found that operating an UGH at a high setting releases around a half litre of water vapour per hour of use $[14,15]$ with an average vapour pressure increase of $0.01 \mathrm{kPa} / \mathrm{min}$ [8].

Optimal RH is the primary parameter for fungi to grow. When the RH of the air surrounding the building material is in a steady state condition (equilibrium), this RH is called equilibrium RH (ERH) and is equivalent to the water activity $\left(a_{\mathrm{w}}\right)$ of the building material when expressed as a fraction. A building material in a steady state condition with a surrounding $\mathrm{RH}$ of $70 \%(\mathrm{ERH}=70 \%)$, will have $a_{\mathrm{w}}=0.70$. This $70 \%$ ERH threshold was chosen in Table 2, as it represents the minimal ERH $/ a_{\mathrm{w}}$ for xerophilic fungi to start growing [12,16] and two of the three fungal inclusions in the fungal detector contained xerophilic fungi (Eurotium herbariorum and Aspergillus penicilloides).

Table 2 shows the percentage of time, in the living rooms and in the bedrooms, with a wall RH above $70 \%$ for both types of heater being operated in the living rooms during the 41.4 days' period.

Table 2 shows that the bedrooms had greater exposure to $\mathrm{RH}$ levels above $70 \%$ than the living rooms. The households operating an UGH showed a higher percentage of time in both the living rooms and the bedrooms than the households operating a HP. In fact, the psychrometric conditions were substantially different in the UGH and HP homes which should impact on the capacity for mould to grow on the wall surface.

\subsection{Fungal Daily Growth Rate on the Interior Lining of an External Wall Surface}

The 52 fungal detectors (one in each living room and one in each bedroom) were exposed for an average period of 85 days with an exposure range between 62 and 109 days. The exception to this was one dwelling where the fungal detectors stayed for 154 days, due to the householders being unavailable at an earlier time for collection of the slides. Following hyphal measurements under microscope, the daily growth rate outliers were removed from the analysis. The lower outliers were identified as values lower than the value of $(\mathrm{P} 75-((\mathrm{P} 75-\mathrm{P} 25) \times 1.5))$ and the upper outliers were identified as values greater than the value of $(\mathrm{P} 75+((\mathrm{P} 75-\mathrm{P} 25) \times 1.5))$. Wilcoxon's rank tests were applied to test if the daily hyphal growth rate $(\mu \mathrm{m} /$ day) was different between households operating an UGH and households operating a HP. Figure 2 shows the average daily hyphal growth rate in the living rooms and in the bedrooms. 


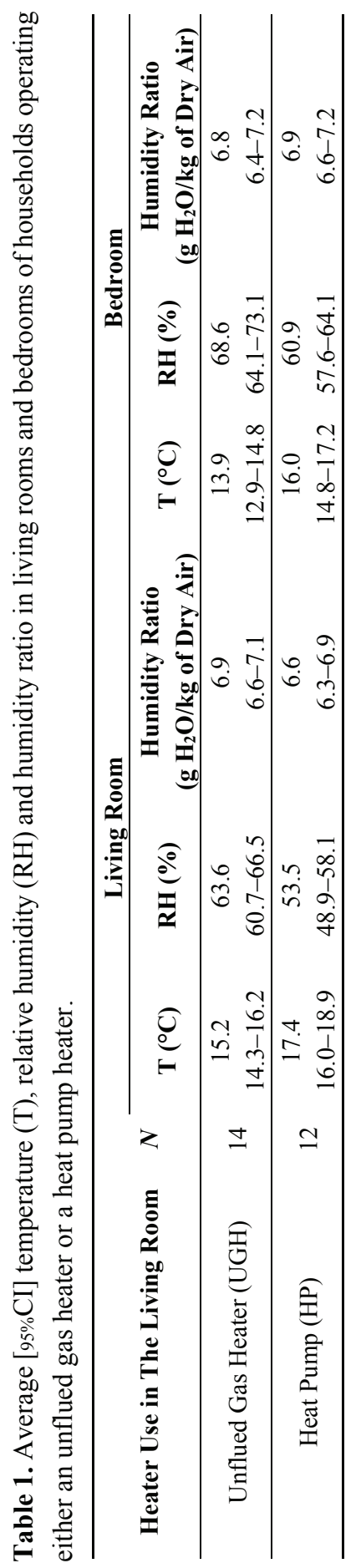


Table 2. Percentage of time with the wall $\mathrm{RH}$ above $70 \%$ in the living rooms and in the bedrooms.

\begin{tabular}{cccc}
\hline \multirow{2}{*}{ Heater Type Used in the Living Room } & \multirow{N}{*}{} & \multicolumn{2}{c}{ Percentage of Time with $\mathbf{R H}>\mathbf{7 0 \%}$ (\%) } \\
\cline { 3 - 4 } & & Living Room & Bedroom \\
\hline Unflued Gas Heater (UGH) & 14 & 29 & 46 \\
Heat Pump (HP) & 12 & 9 & 18 \\
\hline
\end{tabular}

In the livings rooms, the daily hyphal growth rates for both xerophilic fungi (Aspergillus penicilloides and Eurotium herbariorum) were four times higher in the UGH user group than in the HP user group, however these results were not statistically significant due the small sample size. The daily hyphal growth rate for Alternaria alternata was slightly higher in households operating a HP than in household operating an UGH $(0.90 \mu \mathrm{m} /$ day (HP) $v s .0 .77 \mu \mathrm{m} /$ day $(\mathrm{UGH}), p=0.07)$. In the bedrooms, the daily hyphal growth rates for both xerophilic fungi were 7 and 26 times higher in households operating an UGH than in households operating a HP respectively $(p<0.01)$. No significant differences were found for Alternaria alternata between both household groups in the daily hyphal growth rates.

The climate was found to be more suitable for mould development in the bedrooms than in the living rooms. These results are consistent with the percentage of time that the RH was above $70 \%$, being twofold higher in the bedrooms than in the living rooms (Table 2). A factor contributing to this difference in bedroom climates was the effect of the heater operated in the living rooms; the HP seems to be more effective than UGH in heating the bedrooms (forced air circulation due to HP fan). Boulic et al. [5] found that UGHs were mainly operated in NZ on low or medium setting which gives an estimated heat output of $2.6 \mathrm{~kW}$. Since most UGH appear to have a nominal heating capacity of $4 \mathrm{~kW}$, that suggests an element of underuse by occupants and as well as a heater capacity issue.

However, the more favourable condition for fungi to grow, in households who operated an UGH, was only true for the two xerophilic fungi Eurotium herbariorum and Aspergillus penicilloides. Consistent with the fact that these two fungi are the first to react to environment changes, they are considered as first colonizers because they can grow under relatively "dry" conditions $\left(a_{\mathrm{w}}>0.70\right)$ whereas hydrophilic fungi like Alternaria alternata need very humid conditions $\left(a_{\mathrm{w}}>0.90\right)$ to start germination $[12,16,17]$. Such conditions were infrequent in all studied houses, even those with UGHs. Abe et al. [18] found higher hyphal growth rate for Eurotium herbariorum in water associated rooms than in other rooms, showing a positive correlation between high moisture source and high hyphal growth rate. However, when comparing Eurotium herbariorum and Alternaria alternata, the same authors found a higher response for Alternaria alternata in water usage area (bathroom) [12]. 


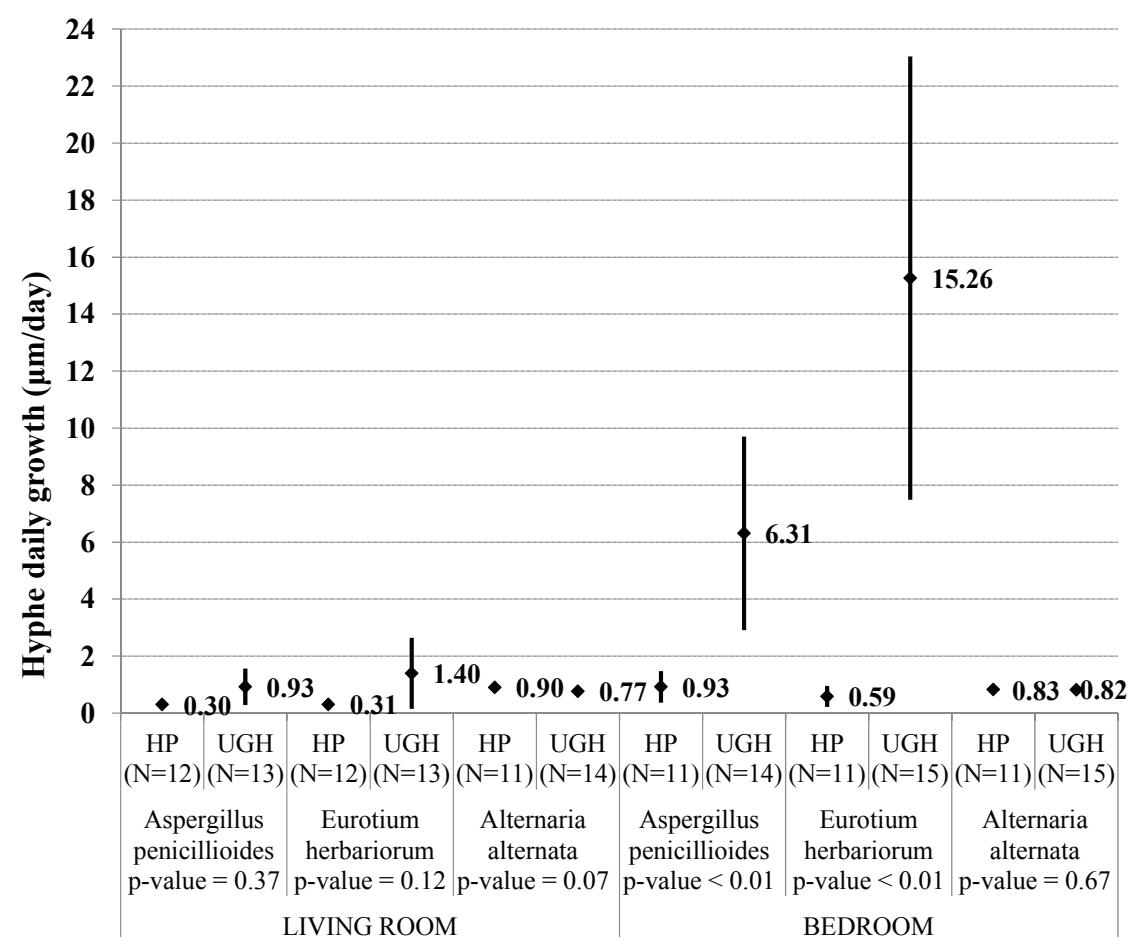

Figure 2. Average daily hyphal growth rate and lower and upper $95 \%$ confidence limit in the living rooms and in the bedrooms (outliers removed).

\subsection{Fieldwork Hyphal Development Compared to Laboratory Hyphal Development}

Average temperature and RH were plotted on a climograph (isopleths) for Eurotium herbariorum (Figure 3), and Alternaria alternata (Figure 4). The isopleth for Aspergillus penicilloides is similar to the Eurotium herbariorum isopleth as both fungi have very similar temperature/RH requirements; therefore the Aspergillus penicilloides isopleth is not shown. The temperature/RH values found in the fieldwork were grouped as "UGH living room", "UGH bedroom", "HP living room" and "HP bedroom" and were compared to optimum climate zone for fungal germination which were obtained under constant conditions of temperature/RH in a laboratory. The laboratory data shown in Figure 3 were adapted from published work [13]. The laboratory data shown in Figure 4 were provided by Abe and are unpublished [19].

In Figure 3, the 8 to 30 days germination zone (black triangle) starts at $\mathrm{RH}=70 \% / T=25{ }^{\circ} \mathrm{C}$. Figure 3 shows that the average climate in the UGH user group (dashed ellipse) was closer to the germination zone than in the HP user group (plain ellipse). Six bedrooms and two living rooms from the UGH user group had an averaged climate that was either close or within the 8 to 30 days germination zone, whereas no room from the HP user group was within this germination zone. For both groups of households (UGH and HP users), the conditions for mould to grow were more favourable in the bedrooms than in the living rooms. 


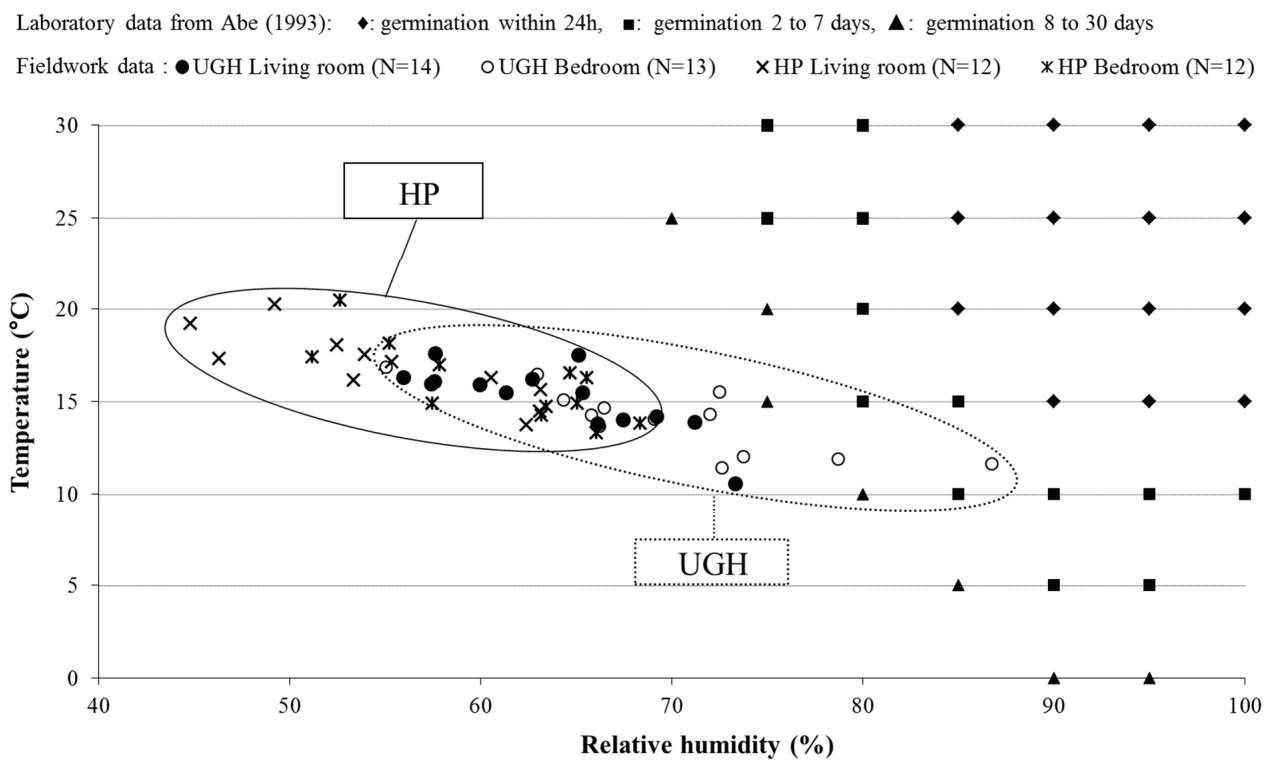

Figure 3. Climograph with germination of Eurotium herbariorum spores; fieldwork data compared to laboratory data (adapted from [13]).

Figure 4 shows different requirements in terms of temperature/RH for the growth of the hydrophilic fungus Alternaria alternata. In Figure 4, the germination zone (black square) starts at $\mathrm{RH}=90 \%$. None of the households from either group was within this germination zone. One bedroom from the UGH user group showed an average RH of $86.8 \%$ which is close to the germination zone but did not show higher hyphal development than the group's average hyphal length. These results are consistent with the very low daily hyphal growth found in both groups for Alternaria alternata (Figure 2).

These results support the previous findings that the daily hyphal growth rates, for both xerophilic fungi (Eurotium herbariorum and Aspergillus penicilloides), were higher in the bedrooms than in the living rooms and also higher in the households operating an UGH than in households operating a HP. The measured temperature/RH values were well below the laboratory germination zone for the hydrophilic fungus Alternaria alternata development $(\mathrm{RH}=90 \%)$, consistent with a low daily hyphal growth rate found for this fungus. 


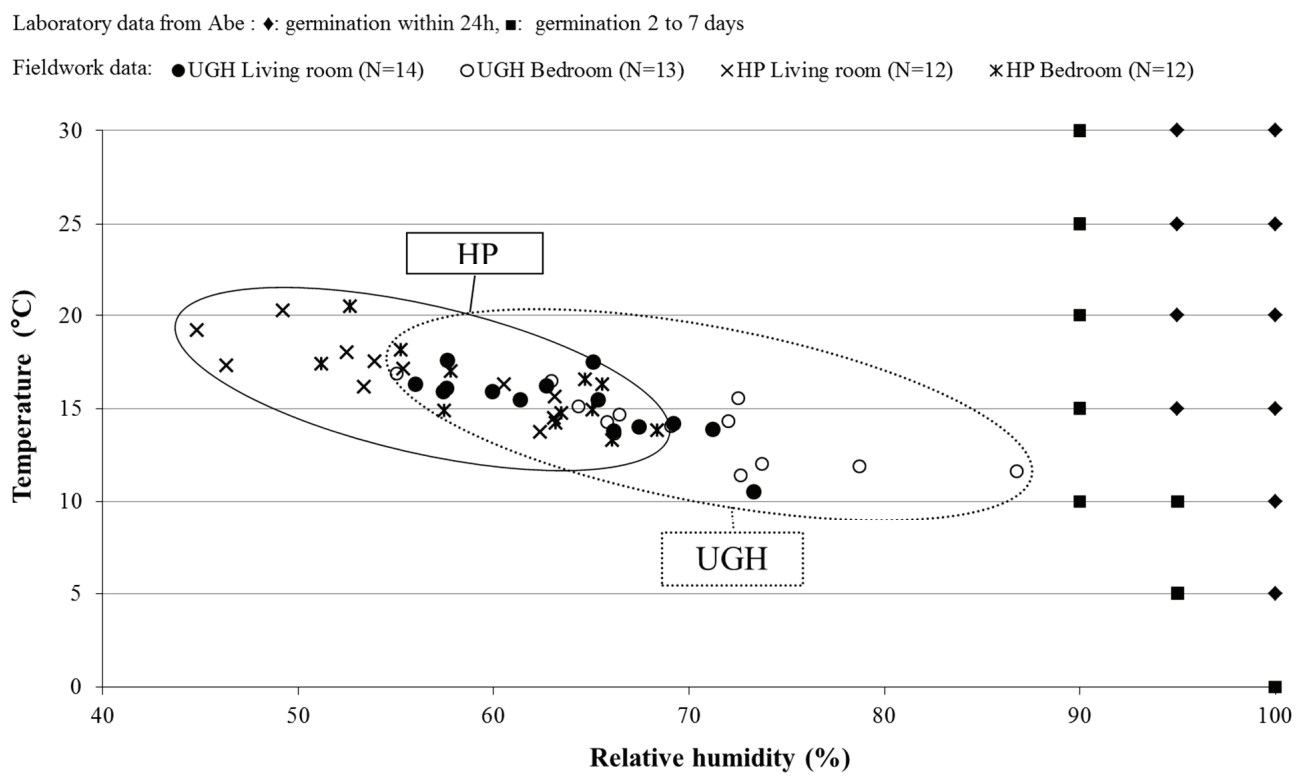

Figure 4. Climograph with germination of Alternaria alternate spores; fieldwork data compared to laboratory data (collected by Abe [19]).

\subsection{Hyphal Development in Response to Favourable Climate Exposure}

The measured daily hyphal growth rate was compared to the time of exposure in favourable psychrometric conditions. This analysis was done using a methodology developed by the Building Research Association of New Zealand [20]. The wall temperature from 0 to $30{ }^{\circ} \mathrm{C}$ and the RH from $35 \%$ to $100 \%$ were divided into $5{ }^{\circ} \mathrm{C}$ and $5 \% \mathrm{RH}$ ranges, respectively. Next, "bins" were created for each $5{ }^{\circ} \mathrm{C}$ and $5 \% \mathrm{RH}$ increment. For example, the temperature and $\mathrm{RH}$ combination of $0-5{ }^{\circ} \mathrm{C}$ and $35 \%-40 \%$ RH was the first of the 78 bins. Spearman's rank correlation tests were applied to test the correlation between the time of exposure in the defined bin and the measured daily hyphal growth rate for all three fungi.

Living rooms and bedrooms with a high measured hyphal growth rate (above the 75th percentiles) were selected for the analysis as these living rooms and bedrooms gave the best fungal development in response to the psychrometric conditions. A total of 13 rooms were selected consisting of 9 bedrooms and 4 living rooms in order to compare the Eurotium herbariorum hyphal development to climate exposure. A total of 14 rooms were selected consisting of 10 bedrooms and 4 living rooms to compare the Aspergillus penicilloides hyphal development to climate exposure, and a total of 11 rooms were selected consisting of 6 bedrooms and 5 living rooms to compare the Alternaria alternata hyphal development to climate exposure.

For Eurotium herbariorum $(N=13)$, the strongest positive correlation value $\left(R^{2}=0.40, p=0.02\right)$ was detected for the bin $10-15{ }^{\circ} \mathrm{C}$ and $80 \%-85 \%$ RH. For Aspergillus penicilloides $(N=14)$, the strongest positive correlation value $\left(R^{2}=0.28, p=0.05\right)$ was detected for the bin $15-20{ }^{\circ} \mathrm{C}$ and 
$85 \%-90 \%$ RH. For Alternaria alternata, no significant positive correlation between the measured hyphal growth rate and any climate bin were detected $(p=0.54)$.

Results show that in the rooms where Eurotium herbariorum had the largest hyphal development, the RH was above $80 \%$ for an average of $4.4 \mathrm{~h}$ a day. This result is consistent with a study by Cunningham [20] which found that Eurotium herbariorum needed to be exposed for at least $3.6 \mathrm{~h}$ per day with a RH above $80 \%$ for hyphal development. Similarly, the rooms where Aspergillus penicilloides showed the largest hyphal development had a $\mathrm{RH}$ above $85 \%$ for an average of $1.9 \mathrm{~h}$ a day.

Results show that in the rooms where Alternaria alternata had the largest hyphal development, the RH was between $35 \% \mathrm{RH}$ and $70 \% \mathrm{RH}$ for $77 \%$ of the time. This climate was too dry for this hydrophilic fungus to develop. The climate was suitable for Alternaria alternata (above 90\% RH) for only an average of $0.1 \mathrm{~h}$ a day ( $0.4 \%$ of the time). This finding supports the lack of correlation found between the daily growth rate and the climate exposure, and was consistent with the low daily hyphal growth rate found for this fungus (Figure 2).

\subsection{Impact of the Heater Choice on the Visible Fungal Level}

Figure 5 shows the percentage of living rooms and bedrooms assessed at each of the four mould indexes in the households either operating an UGH or a HP.

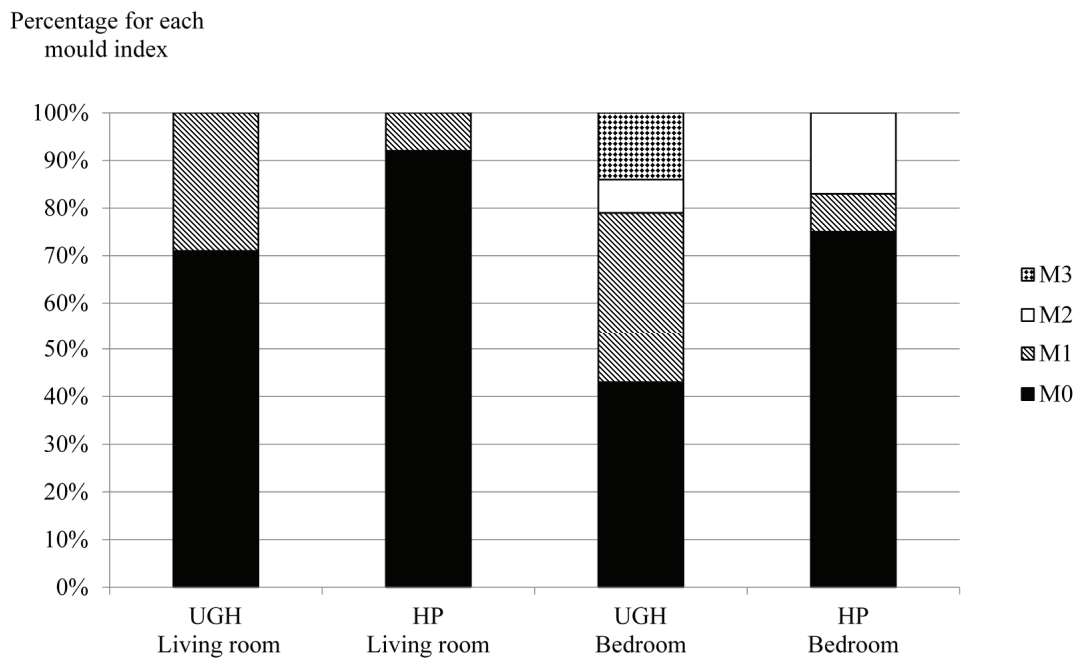

Figure 5. Mould index per heater type (UGH: Unflued Gas Heater or HP: Heat Pump) in the living rooms and in the bedrooms (M0: "no visible mould", M1: "specks of visible mould", M2: "moderate visible mould patches", and M3: "extensive covered areas").

Figure 5 shows a higher percentage of houses being assessed with the "M0" mould index (no visible mould) where HP have been operated ( $92 \%$ vs. $71 \%$ in the living rooms and $75 \% v s .43 \%$ in the bedrooms), compared to the houses where UGH have been operated. 
Spearman's rank correlation tests were applied to test the correlation between the visual inspection mould index (M0, M1, M2 or M3) and the measured daily hyphal growth rate for all three fungal inclusions (Aspergillus penicillioides, Eurotium herbariorum and Alternaria alternata).

A positive correlation $\left(R^{2}=0.16, p<0.01\right)$ was found between the visible mould index in living rooms/bedrooms and the measured daily hyphal growth rate for Aspergillus penicilloides. A similar result was found for Eurotium herbariorum $\left(R^{2}=0.16, p<0.01\right)$, however no correlation was found between the visible mould index in living rooms/bedrooms and the measured daily hyphal growth rate for Alternaria alternata $(p=0.39)$. In conclusion, for Aspergillus penicillioides and Eurotium herbariorum, we can assume that the more visible mould, the higher the daily hyphal growth will be. Another study reported a positive correlation between a high visual fungal contamination and a high hyphal growth rate for Eurotium herbariorum [18].

A positive correlation was also found between the average wall $\mathrm{RH}$ and the visible mould index $\left(R^{2}=0.16, p<0.01\right)$ and a negative correlation between the average wall temperature and the assessed mould index $\left(R^{2}=(-) 0.14, p<0.01\right)$. In the rooms with a contamination level of M0, M1, M2 and M3, the RH was on average $61.5 \%, 68.2 \%, 70.4 \%$ and $71.5 \%$, respectively. The temperature decrease, correlated to an increase of the visible mould index, is consistent with a RH increase at the same humidity ratio. These results are consistent with a needed threshold of $70 \%$ for xerophilic fungi to start growing.

The visible mould level was found to be higher than the level reported from a self-reported NZ telephone survey [2]. The results showed that the visible mould level and the RH level were positively correlated and that the visible mould level and the temperature were negatively correlated. These findings are consistent with a higher percentage of visible mould detected in households where UGHs were operated. Garrett et al. [21] reported a correlation between evidence of dampness and visible mould. Another study found fungal levels were positively correlated with basement humidity [22] and negative correlation was found between the temperature of the room and the fungal level [23].

A meta-analysis showed strong associations between home dampness and respiratory/allergy effects, but the mechanisms linking the specific causal dampness and the related agents are still not clarified [24]. Mould and other microbiological organisms are probably the link between dampness and adverse health effects [25].

\section{Conclusions}

The households operating an UGH experienced a significantly different wall climate than the households operating a HP. In both the living rooms and the bedrooms, the percentage of time with the RH above $70 \%$ was much higher in homes where UGHs were operated than in homes where HPs were operated.

The fungal detectors were useful to predict the capacity for three species of fungi to grow on the inside surface of an external wall. The results showed that the bedroom climate was more suitable for xerophilic mould growth than the living room climate with a much higher hyphal development found in houses where UGHs were operated. These results were supported with a positive correlation between the hyphal development and the time of exposure in the germination psychrometric 
condition zones. However, it was found that the wall RH levels were too low and therefore not suitable for hydrophilic fungus development like Alternaria alternata.

Results from the visual inspection of mould growth were found to be very consistent with the predictions from the fungal detector method. A positive correlation was found between visible mould index and the measured daily hyphal growth rate for both xerophilic fungi (Aspergillus penicillioides and Eurotium herbariorum). However, it should be noted that the visual inspection is a very subjective method of mould assessment which could be influenced by several confounding factors such as the household mould cleaning, the colour of the wall surface and the age of the building material. In conclusion, the fungal detector method is a good complementary method to the visible inspection.

This intervention study had a positive impact on the wall psychrometrics with reduced water availability for mould to grow. The use of UGH was found to be a significant additional source of moisture in the living rooms. It was apparent that UGH user behaviour had an impact on the capacity to heat more than one room in the house. The households were asked to not modify their behaviour in terms of heater usage and indoor activities. The intervention was on the "intention to treat" rather than a "treatment"; consequently the households did not receive any fuel subsidies to encourage the usage of their heater. A follow up study, to investigate the environmental and health effects when a fuel subsidy assists households to pay for a higher consumption of heating could be interesting.

\section{Acknowledgments}

The hyphal measurements were undertaken thanks to the microscope facilities in the Institute of Veterinary, Animal, and Biomedical Sciences, Massey University, Palmerston North, New Zealand.

Participating families from Hutt Valley and community workers from Waiwhetu Marae, Kokiri Marae and Mid Valley Primary Health Organisation are thanked for their valuable contribution to the study. This study was funded by Health Research Council of New Zealand and Building Research Ltd.

In addition to the named authors, the Housing and Health Research Programme consisted of: Michael Baker, Chris Bullen, Julian Crane, Chris Cunningham, Sarah Free, Julie Gillespie-Bennett, Ralph Chapman, Bob Lloyd, Sarah Nicholls, Nevil Pierse, David Shields, Helen Viggers, Kristin Wickens and Alistair Woodward.

\section{Author Contributions}

The paper is based on Mikael Boulic's PhD research work. Robyn Phipps, Malcolm Cunningham and Don Cleland provided supervision and guidance over this research work. All three supervisors reviewed the paper. Pär Fjällström assisted with the study design, the data collection and the review of the paper. Keiko Abe assisted with the data analysis of the fungal detectors and the review of the paper. This research work was a sub study of the Housing, Heating and Health Study which was led by Philippa Howden-Chapman who assisted with the study design and the review of the paper. In addition to the named authors, researchers from the Housing and Health Research Programme reviewed the paper. 


\section{Conflicts of Interest}

The authors declare no conflict of interest.

\section{References}

1. Isaacs, N.; Camilleri, M.; Pollard, A. Housing, Health and Energy. In Housing and Health: Research, Policy and Innovation; Howden Chapman, P., Carroll, P., Eds.; Steele Roberts: Wellington, New Zealand, 2004.

2. Howden-Chapman, P.; Saville-Smith, K.; Crane, J.; Wilson, N. Risk factors for mold in housing: A national survey. Indoor Air 2005, 15, 469-476.

3. Wilton, E. Warm Homes Technical Report: Home Heating Methods and Fuels in New Zealand; Ministry for the Environment: Wellington, New Zealand, 2005.

4. Statistics New Zealand. New Zealand 2013 Census QuickStats about Housing. Available online: http://www.stats.govt.nz/Census/2013-census/profile-and-summary-reports/quickstats-abouthousing/heating-fuels.aspx (accessed on 21 November 2014).

5. Boulic, M.; Fjällström, P.; Phipps, R.; Cunningham, M.; Cleland, D.; Howden Chapman, P.; Chapman, R.; Viggers, H. Cold Homes in New Zealand-Heater Capacity or Low Heater Use? In Proceedings of the SB07 NZ Sustainable Building Conference, Auckland, New Zealand, 14-16 November 2007.

6. Francisco, P.W.; Gordon, J.R.; Rose, B. Measured concentrations of combustion gases from the use of unvented gas fireplaces. Indoor Air 2010, 20, 370-379.

7. Gillespie-Bennett, J.; Pierse, N.; Wickens, K.; Crane, J.; Nicholls, S.; Shields, D.; Boulic, M.; Viggers, H.; Baker, M.; Woodward, A.; et al. Sources of nitrogen dioxide $\left(\mathrm{NO}_{2}\right)$ in New Zealand homes: Findings from a community randomized controlled trial of heater substitutions. Indoor Air 2008, 18, 521-528.

8. Francisco, P.W.; Gordon, J.R.; Rose, W.B. Indoor moisture in 30 homes using unvented gas fireplaces. ASHRAE Trans. 2009, 115, 914-923.

9. Mendell, M.J.; Mirer, A.G.; Cheung, K.; Douwes, J.; Sigsgaard, T.; Bonlokke, J.; Meyer, H.W.; Hirvonen, M.R.; Roponen, M. Chapter 4: Health Effects Associated with Dampness and Mould. Dampness and Mould: WHO Guidelines for Indoor Air Quality; World Health Organization—Regional Office for Europe: Copenhagen, Denmark, 2009.

10. Howden-Chapman, P.; Pierse, N.; Nicholls, S.; Gillespie-Bennett, J.; Viggers, H.; Cunningham, M.; Phipps, R.; Boulic, M.; Fjällström, P.; Free, S.; et al. Effects of improved home heating on asthma in community dwelling children: Randomised controlled trial. Br. Med. J. 2008, 337, $852-855$.

11. NZS 4218:2004 Energy Efficiency-Small Building Envelope; New Zealand Standard: Wellington, New Zealand, 2004.

12. Abe, K. Assessment of home environments with a fungal index using hydrophilic and xerophilic fungi as biologic sensors. Indoor Air 2012, 22, 173-185.

13. Abe, K. A method for numerical characterization of indoor climates by a biosensor using a xerophilic fungus. Indoor Air 1993, 3, 344-348. 
14. Camilleri, M.; Isaacs, N.; Pollard, A.; Stoecklein, A.; Tries, J.; Jamieson, T.; Pool, F.; Rossouw, P. Energy Use in New Zealand Households: Report on the Year 4 Analysis for the Household Energy End-Use Project (HEEP); Study Report SR 98; BRANZ: Porirua, New Zealand, 2000.

15. Tenwolde, A.; Pilon, C.L. The Effect of Indoor Humidity on Water Vapor Release in Homes. In Proceedings of Thermal Performance of the Exterior Envelopes of Whole Buildings $\mathrm{X}$ Conference, Clearwater Beach, FL, USA, 2-7 December 2007.

16. Flannigan, B.; Miller, J.D. Microbial Growth in Indoor Environments. In Microorganisms in Home and Indoor Work Environments: Diversity, Health Impacts, Investigation and Control, 2nd ed.; Flannigan, B., Samson, R.A., Miller, J.D., Eds.; Taylor \& Francis: London, UK, 2011.

17. Darby, J.A.; Caddick, L.P. Review of Grain Harvest Bag Technology under Australian Conditions. A Comprehensive Analysis and Field Evaluation of Harvest Bag Technology: Incorporating a Review of Hermetic and Temporary Storage, Control of Insects and Fungi, and Preservation of Grain Quality, under Typical Australian Storage and Handling Conditions; Technical Report—No. 105; CSIRO Entomology: Clayton South, Australia, 2007.

18. Abe, K.; Nagao, Y.; Nakada, T.; Sakuma, S. Assessment of indoor climate in an apartment by use of a fungal index. Appl. Environ. Microbiol. 1996, 62, 959-963.

19. Abe, K. Institute of Environmental Biology, JDC Corporation, Kanagawa, Japan. Personal communication, 2006.

20. Cunningham, M.J. Final Report on an Alternative Solution to NZBC E3 Field Validation and Model Modification; BRANZ: Porirua, New Zealand, 2001.

21. Garrett, M.H.; Rayment, P.R.; Hooper, M.A.; Abramson, M.J. Indoor airborne fungal spores, house dampness and associations with environmental factors and respiratory health in children. Clin. Exp. Allergy 1998, 28, 459-467.

22. Dekoster, J.A.; Thorne, P.S. Bioaerosol concentrations in noncomplaint, complaint, and intervention homes in the midwest. Am. Ind. Hyg. Assoc. J. 1995, 56, 573-580.

23. O’Connor, G.T.; Walter, M.; Mitchell, H.; Kattan, M.; Morgan, W.J.; Gruchalla, R.S.; Pongracic, J.A.; Smartt, E.; Stout, J.W.; Evans, R. Airborne fungi in the homes of children with asthma in low-income urban communities: The inner-city asthma study. J. Allergy Clin. Immunol. 2004, 114, 599-606.

24. Mendell, M.J.; Mirer, A.G.; Cheung, K.; Tong, M.; Douwes, J. Respiratory and allergic health effects of dampness, mold, and dampness-Related agents: A review of the epidemiologic evidence. Environ. Health Perspect. 2011, 119, 748-756.

25. Fisk, W.J.; Lei-Gomez, Q.; Mendell, M.J. Meta-analyses of the associations of respiratory health effects with dampness and mold in homes. Indoor Air 2007, 17, 284-296. 


\title{
Impacts of an Innovative Residential Construction Method on Internal Conditions
}

\section{Roger Birchmore, Andy Pivac and Robert Tait}

\begin{abstract}
New Zealand houses are known for producing sub-optimal internal thermal conditions and unacceptably high internal moisture levels. These contribute to poor levels of health, mould and can coincide with the decay of structural timber frames. A proposed solution is to provide an alternative structure utilising plywood, a vapour check on the internal face of the timber frame and an additional air gap, followed by the internal lining. The internal vapour check is designed to prevent moisture vapour diffusion from inside into the frame and to permit moisture diffusion from outside through the structure to the internal environment. Two full scale houses had temperatures, dew points and humidity levels monitored in passive, unoccupied conditions. The test case house incorporated the innovative construction solution. The control house was of identical design and location, using standard construction practice. The calculated internal moisture content profile appeared to be unrelated to the external moisture content as expected, instead following the profile of the changing internal temperature. Whilst the innovative construction appeared to prevent moisture diffusion into the structure in winter and permit it inside in summer, this resulted in a generally higher internal relative humidity than the control house.
\end{abstract}

Reprinted from Buildings. Cite as: Birchmore, R.; Pivac, A.; Tait, R. Impacts of an Innovative Residential Construction Method on Internal Conditions. Buildings 2015, 5, 179-195.

\section{Introduction}

Mackintosh [1] summarises New Zealand's climate as:

"Warm subtropical in the far north to cool temperate climates in the far south, with severe alpine conditions in the mountainous areas. Mean annual temperatures range from $10{ }^{\circ} \mathrm{C}$ in the south to $16{ }^{\circ} \mathrm{C}$ in the north of New Zealand. Most of New Zealand would have at least 2000 sunshine hours annually."

Auckland, New Zealand's largest centre of population, located in the north island experiences an average of 632 degree days (base $15.5^{\circ} \mathrm{C}$ ). In the alpine region in the South Island, Queenstown experiences an average of 2137 degree days to the same base [2].

This data does not describe harsh external conditions for much of the population but instances where combinations of low temperatures and high moisture levels lead to poor internal environments, in many parts or the country are documented widely by a number of authors [3-6]. The World Health Organisation [7] links poor internal conditions to a range of health problems that are also reported in New Zealand research. Reflecting the largely temperate nature of the climate, guidance from the Building Research Association of New Zealand (BRANZ) [8] is that vapour barriers are only a requirement in alpine regions or those with significant internal moisture generation linked with spa pools or other similar sources. Moisture in open roof spaces is also not considered to be a significant 
problem and New Zealand Building codes currently have no requirements for the ventilation of roof spaces. Since the publication of the guidance obviating the need for vapour barriers other changes to the Building Code have resulted in minimum insulations levels being raised. There are no requirements for the specific airtightness of buildings. In response to the challenges described, there has been research on solutions that tackle the problems of poor conditions and health directly or indirectly through improving the sustainability of homes [9-12]. This work has tended to focus on the thermal solutions and energy consumption aspects. $\mathrm{Su}[13,14]$ extended this and researched the prevention of winter mould growth in occupied New Zealand houses employing primarily passive and active ventilation and thermal insulation prevention measures. Comparing the static and dynamic simulation methods de Groot [3] expanded further to explore in detail the impacts of moisture transfer through the envelope. He cautioned that the increase of thermal insulation without the consideration of interstitial moisture might move the visible mould problem to an invisible one. Simulating alternative retrofit solutions over a three year period in Auckland, he demonstrated that a vapour barrier was effective in preventing interstitial condensation occurring to levels that might encourage mould growth. Leardini and van Raamsdonk [15] added to the concerns of occupant health to include structural degradation. They also outline fears that increasing levels of thermal insulation increases chances of interstitial condensation. Their Wärmer und Feuchte instationär (WUFI) [16] software simulation of a timber framed house in Auckland, retrofitted with insulation, indicated a clear risk of interstitial conditions when examined over a three year period. This analysis also indicated the tendency for humid summertime conditions to drive moisture inside. The conventional vapour barrier approach risks trapping this moisture vapour into the structure as shown in Figure 1B. They propose that a solution is to provide a vapour check that prevents vapour transfer from moist inside conditions into the wall structure (Figure 1A,C) but also permits externally driven vapour to pass through the structure to the inside (Figure 1D). This vapour check additionally provides all the benefits of an airtight barrier, so whilst reducing the possibility of interstitial condensation, may exacerbate the challenge of increased internal moisture levels and its associated risks. De Groot and Leardini [17] identified a lack of information on the success of retrofit solutions and the general need to improve understanding of the impacts of combining insulation airtightness and humidity control. This paper outlines the early findings of a research project that moves research from desktop simulation to exploring the impact of a construction employing such a vapour check on unoccupied conditions in a real house. 


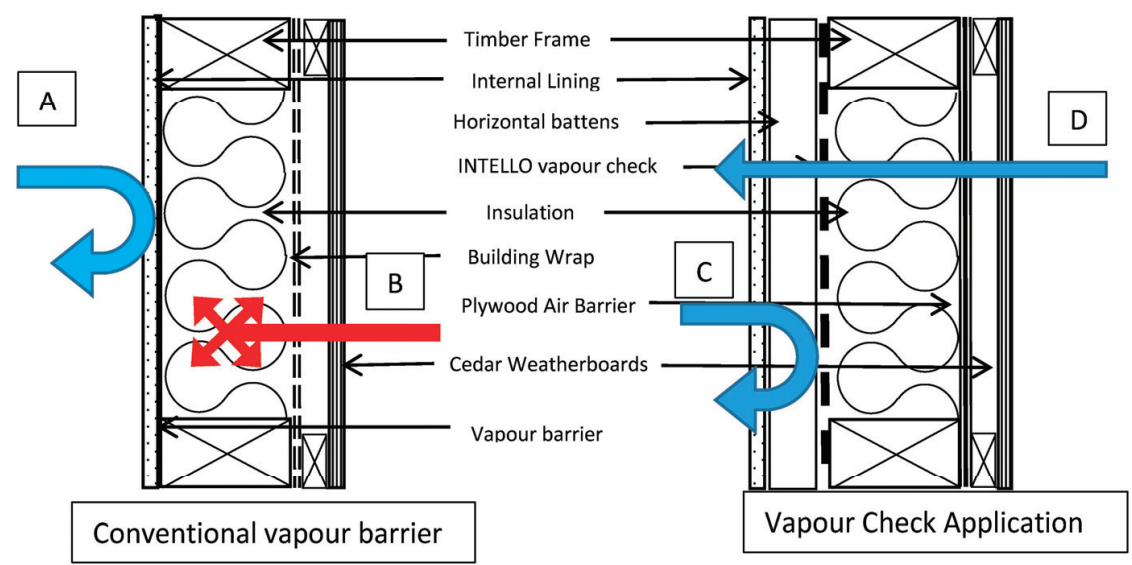

Figure 1. Moisture flows through the structure.

\section{Research Design and Methods}

The fundamental aim of this project is to allow comparison testing of individual or combinations of building materials and techniques that have the potential for improving the building performance of this standard New Zealand house type. In particular, this paper compares the use of a vapour check material not widely used in New Zealand, combined with a rigid air barrier, compared to conventional New Zealand construction methods. The particular focus is on impacts on the moisture levels and internal air temperatures. Initially, manual calculations employing the dew point method were conducted to investigate the likelihood of interstitial condensation occurring in the control house. Figure 2 shows the risk of condensation occurring in the structure $100 \mathrm{~mm}$ from the inside surface. Figure 3 shows how the vapour check removes this risk. This aligns with the more extensive analysis by Leardini and van Raamsdonk [15]. It was decided to employ the vapour check proposed in their work with a view to initially exploring its impact on the internal spatial conditions of the houses. $[18,19]$ indicate the significant impact that occupants can have on the energy consumption and internal conditions of a space, quoting examples where variations were many times the base consumption figures. The two houses have therefore been tested in passive, unoccupied conditions to remove as many variables as possible. This data would provide the basis for predicting impacts of the construction methods on internal comfort conditions, risks of mould growth and also spatial heating and cooling energy use. This is the first stage of a project that will be followed with analysis of the construction method on the interstitial conditions. 


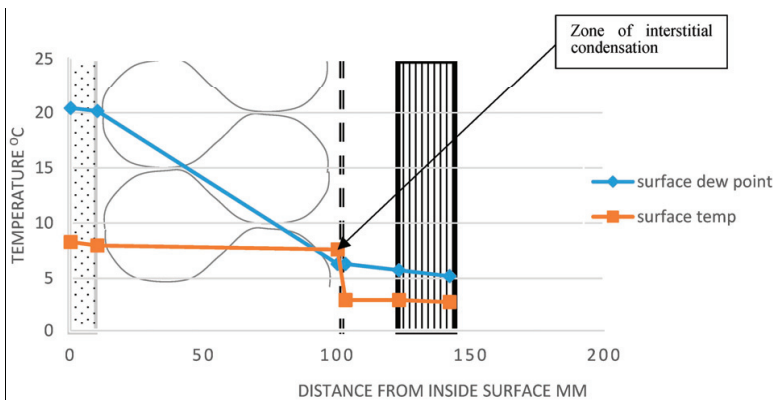

Figure 2. Interstitial condensation calculations in the control house.

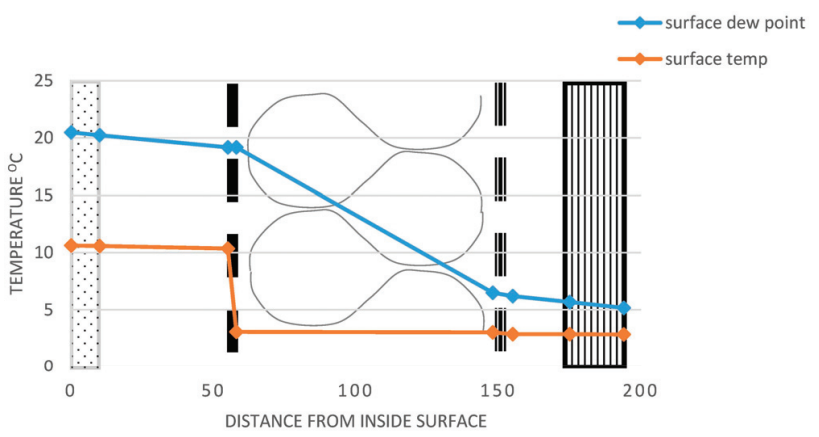

Figure 3. Interstitial condensation calculations in the test house.

\subsection{Control House}

The houses are single storied with three bedrooms and two bathrooms and are constructed as part of the Unitec carpentry programmes. The houses are completed by students to be relocated, and they are undecorated and without floor coverings or wall finishes. Electrical and plumbing fittings are installed but not connected. Figure 4 illustrates their overall nature.

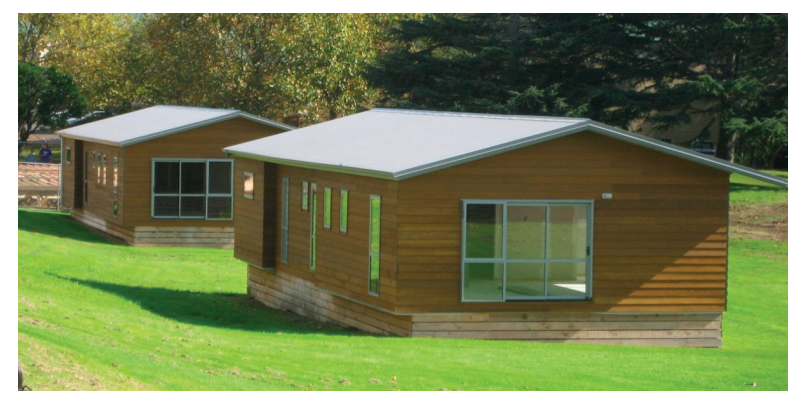

Figure 4. Control house (foreground) and test house.

Table 1 summarises the materials used in the construction of these houses. Overhangs on the north side of the house provide complete shading from direct solar gain through double glazing during the 
hottest periods of the summer months. These houses are similar in design and construction to thousands of houses found in suburban areas and provide an ideal basis for examining the potential for improvements to a common housing type.

Table 1. Construction details for the control and test houses.

\begin{tabular}{|c|c|}
\hline \multicolumn{2}{|c|}{ Common to Control House and Test House } \\
\hline \multicolumn{2}{|c|}{ Timber Frame on pile foundation } \\
\hline \multicolumn{2}{|c|}{ Sub floor cladding $150 \mathrm{~mm} \times 25 \mathrm{~mm}$ radiata pine boards with $20 \mathrm{~mm}$ gaps } \\
\hline \multicolumn{2}{|c|}{ Particle board, floor foil insulation draped between $190 \mathrm{~mm} \times 45 \mathrm{~mm}$ joists } \\
\hline \multicolumn{2}{|c|}{$140 \mathrm{~mm}$ polyester ceiling batts, $10 \mathrm{~mm}$ plasterboard } \\
\hline \multicolumn{2}{|c|}{ Double glazed windows } \\
\hline Control House & Test House \\
\hline Trussroof (radiata pine treated) Coloursteel & Trussroof (radiata pine treated) Coloursteel roofing on \\
\hline roofing on building paper & building paper, INTELLO wrap on bottom chord of trusses \\
\hline cedar weatherboard cladding, natural finish & cedar weatherboard cladding, natural finish \\
\hline 20 mm cavity battens & $20 \mathrm{~mm}$ cavity battens \\
\hline Building wrap & 7 mm Plywood \\
\hline $90 \mathrm{~mm} \times 45 \mathrm{~mm}$ radiata pine framing & $90 \mathrm{~mm} \times 45 \mathrm{~mm}$ radiata pine framing \\
\hline Polyester insulation & Polyester insulation \\
\hline- & INTELLO Vapour check \\
\hline- & $45 \mathrm{~mm} \times 45 \mathrm{~mm}$ battens \\
\hline $10 \mathrm{~mm}$ plasterboard & $10 \mathrm{~mm}$ plasterboard \\
\hline
\end{tabular}

Table 2 provides dimensional thermal and hygroscopic properties of relevant materials in the external envelopes. These are values associated with readily available material sizes to ensure the overall construction $R$-values exceed minimums stated in verification method H/VM1 [20], ensuring compliance with the New Zealand Building Code. Minimum $R$-values for this context are:
- Roof
$2.9 \mathrm{~m}^{2} \mathrm{~K} / \mathrm{W}$
- Wall
$1.9 \mathrm{~m}^{2} \mathrm{~K} / \mathrm{W}$
- Windows
$0.26 \mathrm{~m}^{2} \mathrm{~K} / \mathrm{W}$
- Floor
$1.3 \mathrm{~m}^{2} \mathrm{~K} / \mathrm{W}$

Table 2. Thermal and hygroscopic properties of materials.

\begin{tabular}{cccccc}
\hline Material & Thickness $\boldsymbol{t}$ & Conductivity $\boldsymbol{k}$ & Resistance $\boldsymbol{R}$ & $\begin{array}{c}\text { Vapour } \\
\text { Resistivity } \boldsymbol{r}_{\mathbf{v}}\end{array}$ & $\begin{array}{c}\text { Vapour } \\
\text { Resistance } \boldsymbol{G}\end{array}$ \\
\hline - & $\mathbf{m m}$ & $\mathbf{W} / \mathbf{m K}$ & $\mathbf{m}^{\mathbf{2}} \mathbf{K} / \mathbf{W}$ & $\mathbf{M N s} \mathbf{\text { gm }}$ & $\mathbf{M N s} / \mathbf{g}$ \\
\cline { 2 - 6 } External Walls & - & - & - & - & - \\
\hline Plasterboard & 10 & 0.22 & 0.05 & 60 & 0.6 \\
Air gap (Test House) & 45 & - & 0.18 & - & 5 \\
Vapour Check (Test House) & 3 & - & 2.20 & - & see Table 3 \\
Insulation & 90 & 0.041 & $\mathrm{n} / \mathrm{a}$ & 2300 & 0.63 \\
Building Wrap (Control House) & 3 & $\mathrm{n} / \mathrm{a}$ & 0.05 & - & 6.9 \\
Plywood Air Barrier & 7 & 0.13 & 0.09 & - & 2.2 \\
Ventilated Air Gap & 20 & - & 0.09 & - & 0.26 \\
Cedar Weatherboard & 19 & 0.11 & &
\end{tabular}


Table 2. Cont.

\begin{tabular}{cccccc}
\hline Material & Thickness $\boldsymbol{t}$ & Conductivity $\boldsymbol{k}$ & Resistance $\boldsymbol{R}$ & $\begin{array}{c}\text { Vapour } \\
\text { Resistivity } \boldsymbol{r}_{\mathbf{v}}\end{array}$ & $\begin{array}{c}\text { Vapour } \\
\text { Resistance } \boldsymbol{G}\end{array}$ \\
\hline- & $\mathbf{m m}$ & $\mathbf{W} / \mathbf{m K}$ & $\mathbf{m}^{2} \mathbf{K} / \mathbf{W}$ & $\mathbf{M N s} / \mathbf{g m}$ & $\mathbf{M N s / g}$ \\
\hline Roof Structure & - & - & - & - & - \\
\hline Plasterboard & 10 & 0.22 & 0.05 & 60 & 0.6 \\
Air gap (Test House) & 45 & - & 0.18 & - & 5 \\
Vapour Check (Test House) & 3 & - & - & - & see Table 3 \\
Air Gap & 300 & - & 0.16 & - & 5 \\
Building Wrap & 3 & - & - & 2300 & 6.9 \\
Profiled Sheet Steel Roof & 4 & - & - & - & 2000 \\
\hline
\end{tabular}

\subsection{Test House}

The test house was constructed one year later than the control house and modifications incorporated the use of the INTELLO vapour check proposed by Leardini and van Raamsdonk [15]. In addition modifications were made were to replace the building paper with $7 \mathrm{~mm}$ thick plywood barrier treated to H3.2 CCA (Copper Chrome Arsenate) in accordance with AS/NZ 1604.3 [21] to meet AS/NZS 2269.0 [22]. Vertical sheet joints were sealed with flashing tape. This feature was felt to have significant potential as an alternative that provided the functions of bracing and rigid air barrier in a single element that offered enhanced seismic resistance. This had potential for use in the rebuild of houses since the Christchurch earthquakes. The vapour check was placed on the internal surfaces of external walls and ceilings. A $45 \mathrm{~mm}$ cavity batten was then added before fixing of the plasterboard. Details are shown in Figures 5 and 6. Ceiling insulation was placed directly on top of the ceiling material in the control house and on top of the INTELLO vapour check in the control house. In both houses the roof space that resulted was an uninsulated cavity. The INTELLO vapour check is intended to have two functions. The first is to control the passage of vapour through the timber to the structure. This minimises the chances of charging the frame with moisture that could then condense during cold spells, eventually resulting in the rotting of the frame. Its high level of resistance shown in Table 3 prevents vapour generated internally from passing into the structure in winter. The low level of resistance in the opposite direction permits the vapour to pass from high humidity conditions present in summer time though the structure to the inside. This again prevents the possible accumulation of moisture in the frame for future condensation. The second function is for the INTELLO vapour check to act as an additional barrier to infiltration through the fabric of the building. The minimisation of infiltration is proposed to be an important contributor to reducing unplanned heat loss and moisture ingress. The additional air gap between the INTELLO and the internal plasterboard lining provides a route for internal services to circulate with minimal penetrations through the INTELLO. 


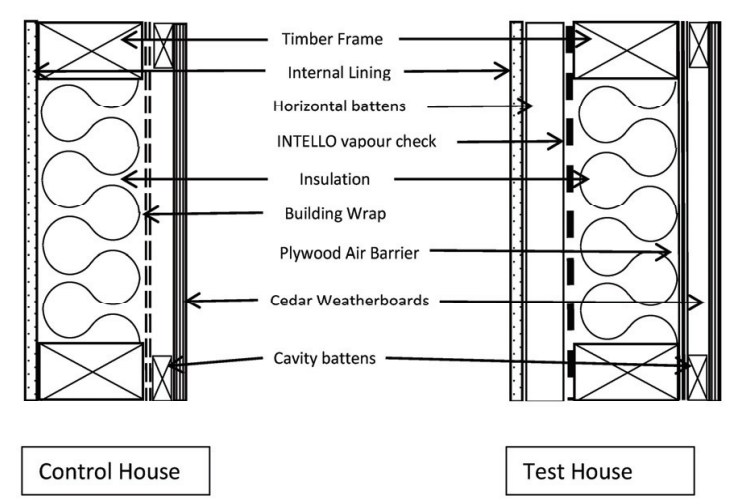

Figure 5. Construction detail—external walls.

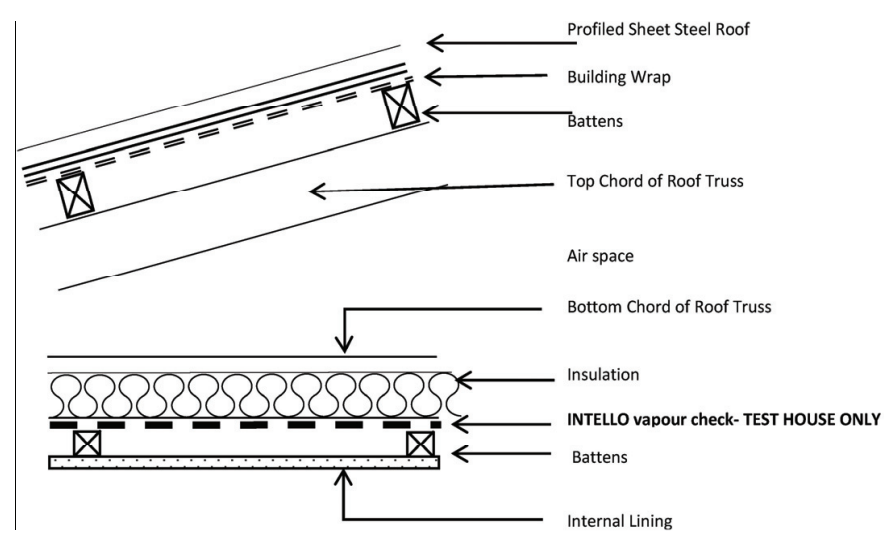

Figure 6. Construction detail—roof.

Table 3. Vapour diffusion resistance of the INTELLO membrane [23].

\begin{tabular}{ccc}
\hline Testing Conditions & \multicolumn{2}{c}{ Vapour Diffusion Resistance (MNs/g) } \\
\hline Average Ambient Humidity & $20 \%$ & $85 \%$ \\
Direction of Diffusion Flow & Out towards the air barrier & Inwards towards the air barrier \\
INTELLO & 125 & 1.25 \\
\hline
\end{tabular}

\subsection{Site}

The site is on the Unitec Institute of Technology campus in Mt Albert Auckland. The site is relatively exposed with an open grassed area to the northwest. Surrounding buildings are reasonably distant to the south, north and east. Behind the houses to the southeast is a hilly incline and the student building yard. The houses are located with identical orientations but separated to avoid mutual shading. 


\subsection{Monitoring Process}

Temperature sensors have been set up to sample the internal air temperature at hourly intervals. Sensors used are Lascar EL-USB-2 Humidity and Temperature USB data loggers. These measure and store relative humidity (RH), dew point (DP) and dry bulb air temperature readings (DB) over $0 \% \mathrm{RH}$ to $100 \% \mathrm{RH}$ and $-35{ }^{\circ} \mathrm{C}$ to $+80^{\circ} \mathrm{C}$ measurement ranges. Sensors were located identically in the two houses to align with practice outlined by Barley et al. [24] at a height of $1500 \mathrm{~mm}$ above ground level suspended from the ceiling by builders twine. Sensor layout is given in Figure 7. In order to check the appropriate test location for the sensor, a second sensor was located at the edge of the room to check initial operation and determine the degree of variability experienced across each space. It was found that the average variation between measurements from the centre of the room and from the edge of the room vary by an average of $0.2{ }^{\circ} \mathrm{C}$ over the 168 hourly measurements, with the maximum variation less than $0.5^{\circ} \mathrm{C}$. This is well within the accuracy stated for the sensors which is stated to be $\pm 0.5^{\circ} \mathrm{C}$ [25] and indicates that a single measurement in the chosen position is representative of the overall room conditions. Dew point measurements have been used as this single figure provides an indicator of absolute moisture content. Localised weather data is measured at a weather station indicated in Figure 8. They are monitored in a passive, unoccupied condition.

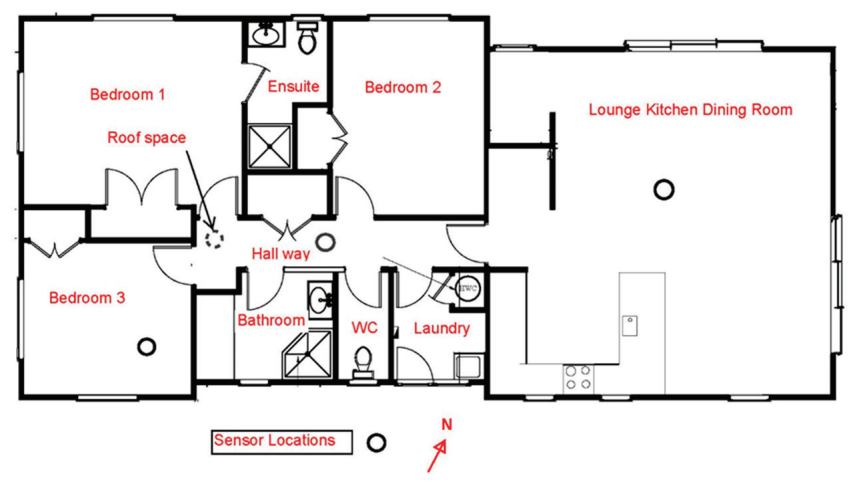

Figure 7. House plan with sensor locations.

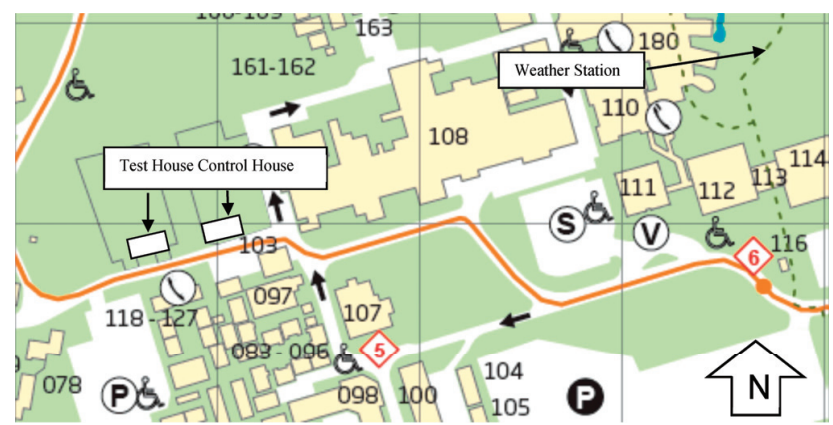

Figure 8. House and weather station location details. 


\subsection{Air Tightness}

Both houses were tested for air tightness using the standard blower door test following European standard EN 13829:2000 [26]. Openings associated with extract ventilation and unconnected waste pipes were sealed for air pressure testing and for subsequent monitoring of air temperature, dew point and relative humidity. Table 4 shows the air changes per hour of the whole house volume under the standard test conditions of $50 \mathrm{~Pa}$ pressurisation and $50 \mathrm{~Pa}$ depressurisation. It indicates that the test house has an air leakage rate of less than a third of the control house. The control house sits just outside the airtight classification for New Zealand houses which peaks at an airtightness of $5 \mathrm{ac} / \mathrm{h}$ [27]. The test house is comfortably in the airtight classification but is still well above the requirements of the Passive House Institute [28].

Table 4. Results of airtightness testing.

\begin{tabular}{ccc}
\hline Testing Conditions & Control House (ac/h) & Test House (ac/h) \\
\hline Depressurisation & 6.58 & 1.92 \\
Pressurisation & 6.93 & 2.10 \\
Average & 6.75 & 2.01 \\
\hline
\end{tabular}

\subsection{Rooms Being Analysed}

The rooms chosen for analysis in this paper were the lounge kitchen dining room, bedroom 3 , hall and the roofspace. Floor and wall areas are shown in Table 5. The lounge kitchen dining room is the largest space in the house and is has external walls on the South, East and North Face with glazing in each with a window to wall ratio of $29 \%$. Its inclusion of the kitchen also examines a space where occupancy may generate significant additional internal moisture. Bedroom 3 is on the opposite, coldest corner of the house receiving minimal solar gain. With a window to wall ratio of $41 \%$ the hall is internal with the exception of its floor and ceiling. The roofspace provides data on the external side of the vapour check in the test house and the external side of the insulation in both houses.

Table 5. Details of rooms being analysed.

\begin{tabular}{ccc}
\hline Room & Floor Area $\left(\mathbf{m}^{\mathbf{2}}\right)$ & Net External Wall Area $\left(\mathbf{m}^{\mathbf{2}}\right)$ \\
\hline Lounge Kitchen Dining room & 46.4 & 33.6 \\
Bedroom 3 & 9.8 & 11.07 \\
Hall & 5.6 & Nil \\
Roofspace & 120.5 & Nil \\
\hline
\end{tabular}

\section{Results and Discussion}

\subsection{Seasonal Data}

The means of dry bulb (DB) and dew point temperatures, (DP) measured at hourly intervals for the winter and summer seasons are summarised for each building in Figure 9. The summer season is defined as 1 December-28 February, spring as 1 March-31 May, winter as 1 June-31 August and spring as 1 September-30 November. The data indicates that as the mean temperatures drop towards 
winter and as expected rise up again in spring. Also as expected, the range of temperatures in the roofspace exceed those in occupied spaces indicating the smoothing effect of in wall and under floor insulation. The trend and interrelationship holds for all three internal spaces suggesting that the airspace in each room within each house is reasonably homogenous and that orientation and wall to window ratios have a small effect on mean temperatures. Absolute moisture content and dry bulb temperature are not parameters that are inherently connected. As the buildings have no internal moisture generation it was expected that absolute moisture contents, represented by the dew point temperature levels would follow external moisture readings. Figure 9 indicates that in fact they seem to follow the internal dry bulb readings. The detail of a few sample days in Figures 10 and 11 re-enforces this and indicates little or no connection between internal and external dew point temperatures.

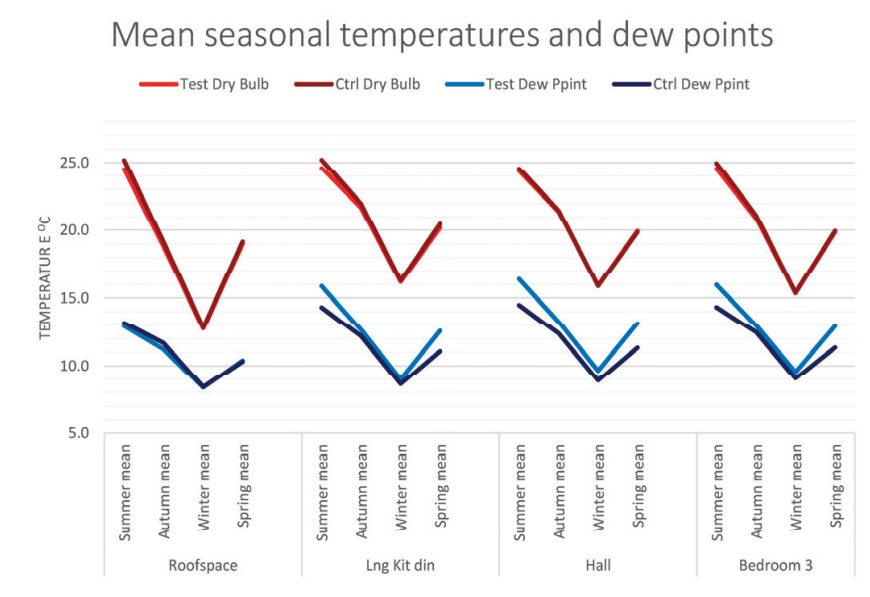

Figure 9. Comparison of space temperature and dew point seasonal means.

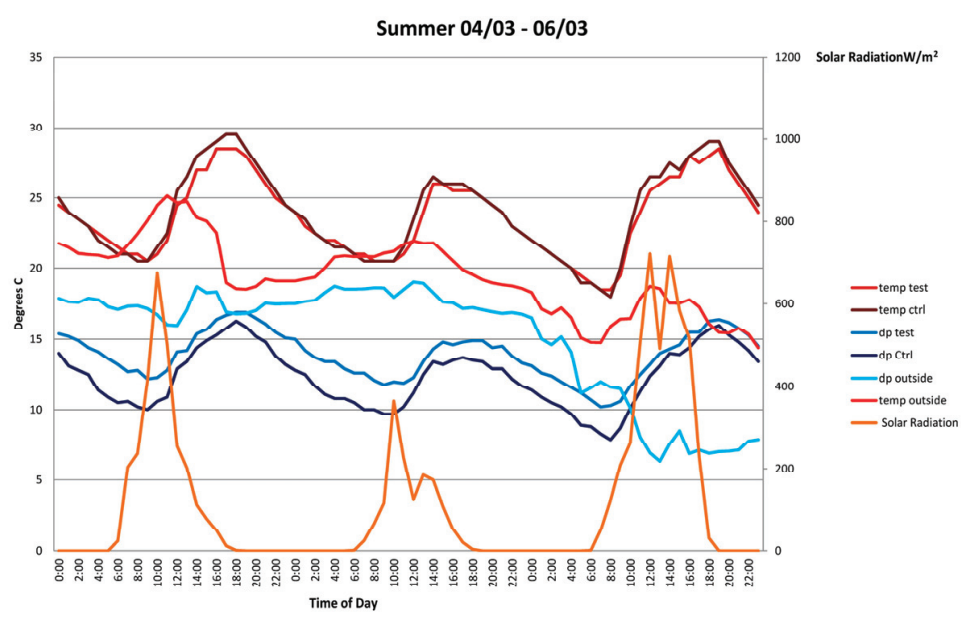

Figure 10. Dry bulb and dew point comparisons for the lounge kitchen dining room with external summer conditions. 


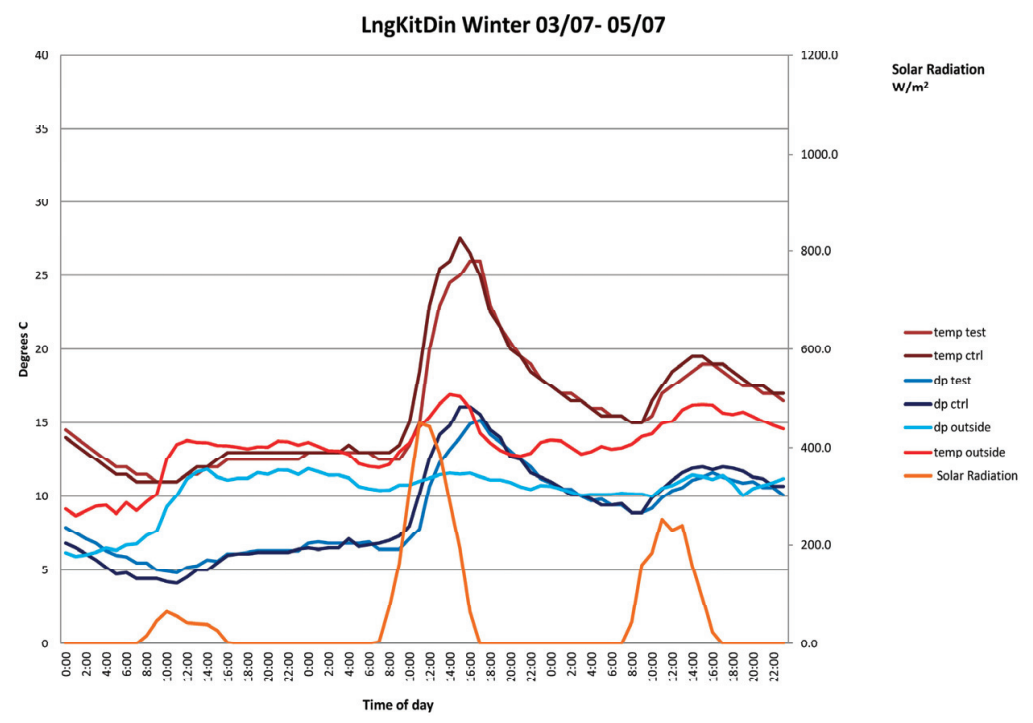

Figure 11. Temperatures and dew point comparisons for the lounge kitchen dining room with external winter conditions.

The significance of the differences between seasonal measures of control and test dry bulb temperatures and between control and test dew point temperatures were calculated using a two tailed $T$-test. Calculations were completed on two spaces, the roofspace and the lounge kitchen dining room being the most different spaces. Calculations were completed on seasonal means using an alpha of 0.05 with a hypothesis that the difference between two population means would be zero. $P$ scores are quoted to four significant figures.

Scores where $p \geq 0.05$ indicates that the hypothesis is correct and that there is no significant difference between the populations. Scores where $p \leq 0.05$ indicates the hypothesis is incorrect and that there is a significant difference between the populations. Table 6 shows that in all spaces with the exception of the roofspace during winter and for dry bulb temperatures in spring the difference between the test and control house was statistically significant with a confidence of $95 \%$.

Table 6. Summary of statistical significance of differences between control and test houses.

\begin{tabular}{cccc}
\hline Season & Space & Dry Bulb & Dew Point \\
\hline \multirow{2}{*}{ Summer } & - & $\boldsymbol{p}$ & $\boldsymbol{p}$ \\
& LoungKitDin & 0 & 0 \\
& Roofspace & 0 & 0 \\
\hline \multirow{2}{*}{ Autumn } & LoungKitDin & 0 & 0 \\
& Roofspace & 0 & 0 \\
\hline \multirow{2}{*}{ Winter } & LoungKitDin & 0 & 0 \\
& Roofspace & 0.1007 & 0.3745 \\
\hline \multirow{2}{*}{ Spring } & LoungKitDin & 0 & 0 \\
& Roofspace & 0 & 0.0263 \\
\hline
\end{tabular}




\subsection{Detailed Results of Selected Days}

\subsubsection{Summer}

The detailed results of a few days in each season are shown in Figures 10-13. They have been chosen to illustrate the apparent strong influence of solar gain on internal conditions. This indicates that the internal dry bulb temperatures follow the cyclical pattern of the external solar gain. The periods of the two cycles appear identical but with the dry bulb lagging between four and $6 \mathrm{~h}$. The internal dew point also follows with the same period but with slightly reduced amplitudes. There is a very weak connection if any, with the dew point of the external air. As there were no occupant generated sources of internal moisture, the changing dew points over a daily cycle could be resulting from residual construction moisture. As the temperature rises, moisture still present in the construction evaporates into the air. As it cools it is re-absorbed into the structure. Whilst the test house vapour check is designed to reduce this, the floors of both houses are exposed particle board and the unpainted gypsum board will have some absorption capacity.

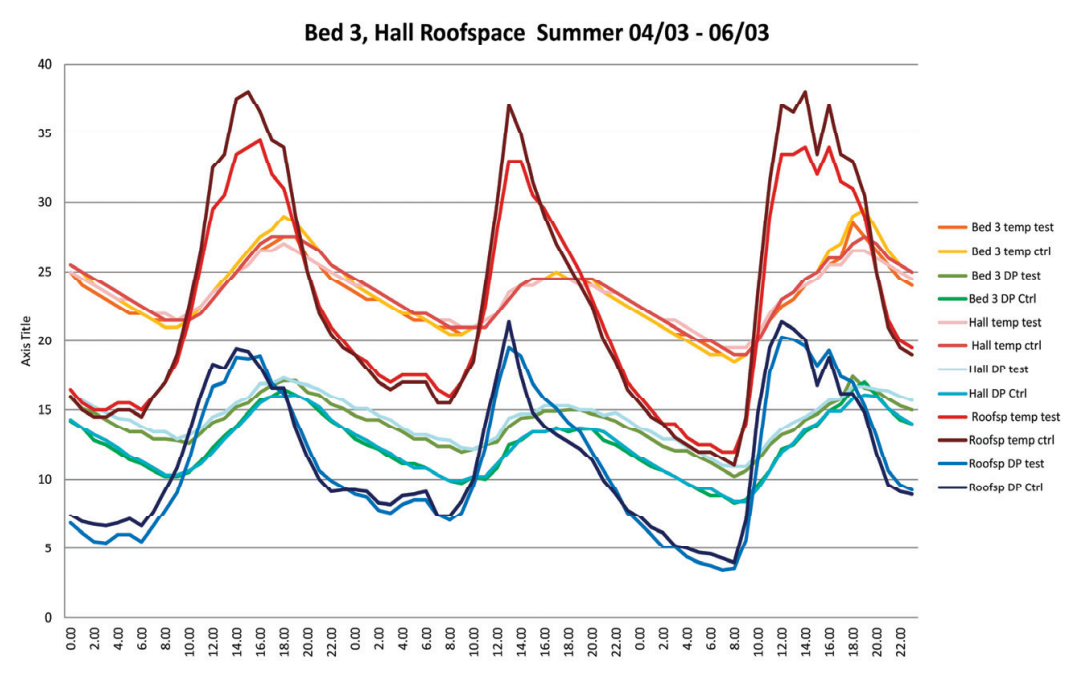

Figure 12. Dry bulb temperatures and dew point comparisons for the bedroom 3 hall and roofspace with external summer conditions.

Figure 12 shows the performance for three other spaces during the same period. The external conditions are the same as in Figure 10 but have been removed for clarity. Reflecting the seasonal means in Figure 9, the roofspace exhibits a wider range of variations than other internal spaces. The dry bulb and dew point temperatures in the bedroom 3 and hall cluster close together within similar ranges and cycle to those of the lounge kitchen dining room. This demonstrates that the seasonal homogeneity is reflected on a daily cycle. The reduction of extreme internal temperatures by thermal insulation in the roofspace is also very clear on this daily cycle. The four to $6 \mathrm{~h}$ lag between peak external temperature and internal temperature observed in the lounge kitchen dining room remains for other internal spaces. This is reduced to two hours for the roofspace. The reduction in lag is 
expected as the temperature is being measured on the external side of the insulation. Differences between dry bulb temperatures in the control house and the test house are small, being largest at extremes of conditions and very small between. Differences between the dew point readings in the houses appear more significant and more evenly spread over the daily cycle.

\subsubsection{Winter}

The data in Figure 11, Illustrates the strong link between solar gain, internal dry bulb and internal dewpoint temperatures where the solar gain on the second day leads and influences the plots of other parameters. Notably the thermal lag in both houses has reduced to approximately $2 \mathrm{~h}$.

Figure 13 reflects parallels with Figure 12 but with lower temperature levels associated with the winter season. The clustering of the graphs continues to support the homogeneity of the internal spaces. The roofspace extremes are reduced. Time lags have also reduced to a maximum of two $h$ and minimums of zero. Differences between control and test house dry bulb temperatures and dew points are very small especially on the first day with low solar radiation.

Moisture Contents, in kg of moisture per kg of dry air, can be read from a Psychrometric chart for given dew points. Using the mean density of the air and the space volume, the volume of moisture being evaporated and re-absorbed on a daily cycle can be estimated. 1 December was chosen as the day with the maximum daily change in dew point temperature and Table 7 indicates the result as $0.52 \mathrm{~L}$.

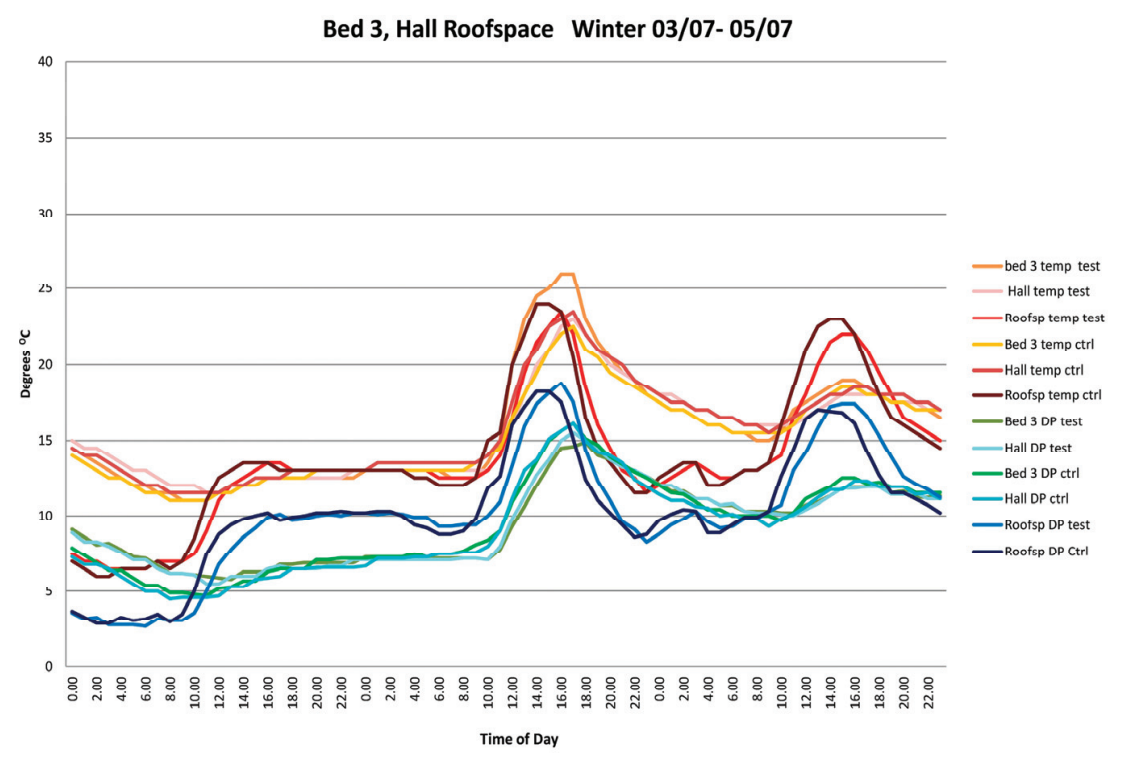

Figure 13. Dry bulb temperatures and dew point comparisons for the bedroom 3 hall and roofspace with external winter conditions. 
Table 7. Data for estimation of the moisture volume being evaporated and re-absorbed.

\begin{tabular}{cccc}
\hline \multicolumn{4}{c}{ Summer 1st Dec } \\
\hline Dry Bulb $\left({ }^{\circ} \mathbf{C}\right)$ & Dew Point $\left({ }^{\circ} \mathbf{C}\right)$ & Density $\left(\mathbf{k g} / \mathbf{m}^{\mathbf{3}}\right)$ & Moisture Content $(\mathbf{k g} / \mathbf{k g})$ \\
\hline Max 25.5 & 15.4 & 1.163 & 0.0109 \\
Min 15.5 & 8.6 & 1.211 & 0.0069 \\
Difference & 6.8 & - & 0.004 \\
Mean density $\left(\mathrm{kg} / \mathrm{m}^{3}\right)$ & - & 1.187 & - \\
Space volume $\left(\mathrm{m}^{3}\right)$ & 108.54 & - & - \\
Space mass $(\mathrm{kg})$ & 128.809 & - & - \\
Moisture mass $(\mathrm{kg})$ & 0.52 & - & - \\
Moisture volume $(\mathrm{L})$ & 0.52 & - & - \\
\hline
\end{tabular}

\section{Conclusions}

The internal seasonal spatial data in Figure 9 along with the statistical analysis supports the expected performance of the test house structure regarding internal moisture levels. The summer season trends are reinforced by the daily analysis in Figure 10. In winter, higher internal dew points in the test house compared to the control house suggest that internal vapour is held within the occupied space and is not being permitted to enter the structure. The risk of interstitial condensation should therefore be reduced. In summer, higher internal dew points in the test house compared to the control house suggest that vapour is being allowed to pass through the structure to the inside. This prevents the moisture getting trapped within the timber frame, reducing the potential to cause interstitial condensation when the climate permits. The roofspace data supports this further. As the readings are on the external side of the vapour check, vapour passing through the check in summer should result in lower dew point temperatures within the control house roofspace compared to the test house. Figure 10 indicates this. They should also be lower in winter but this is not as evident when looking at seasonal means or the test of significance, but the daily analysis in Figure 12 does support this. Overall this differentiates the performance of the vapour check from a conventional vapour barrier which would trap the moisture within the timber structure, preventing it from entering the occupied space.

Compared to the control house, the test house offered improved thermal resistance from the additional sealed air gap and significantly reduced infiltration due to the vapour check properties. It was felt that this would result in warmer test house conditions in winter and cooler conditions in summer. Overall differences are not large however. Small differences between the average dry bulb temperatures over the winter and spring seasons between the control and test Houses suggest that the different properties are having a small effect on unoccupied thermal conditions. Figure 11 shows that on cloudy winter days the difference between internal and external temperatures was minimal and so differences between control and test houses could also be very small. However on sunny winter days, internal temperatures rose nearly ten degrees higher than outside. Under these winter conditions the test house was actually slightly cooler than the control house. As the test house infiltration rate was one third of the control house rate, this suggests that the heat losses due to infiltration through the 
closed structure in an unoccupied condition might be much smaller than anticipated or are not being prevented by increased airtightness of the envelope.

The calculated daily variation of $0.52 \mathrm{~L}$ of water is a small proportion of the possible moisture generation in a house. TenWolde and Pilon [29] suggest that a family of four would produce up to $15 \mathrm{~L}$ per day. However, $0.52 \mathrm{~L}$ represents the amount of moisture left to be absorbed by the air. The moisture absorptive properties on internal linings, quantified by Mitamura, Rode and Schultz [30] and termed moisture buffering, could account for a larger volume of daily moisture change in the unoccupied condition.

The tendency of the vapour check to produce slightly higher internal dew points emphasises the need to combine this type of structure with minimal ventilation rates to ensure the moisture is ventilated to outside. Internal surface temperatures are likely to be even lower than space temperatures, especially in winter. The combination of this fact and that moisture generated by occupation may aggravate conditions, further underlines the importance of ventilating moisture sources to outside.

Whilst the positive differences in conditions provided by the innovative structure are statistically significant they are still small and might be unlikely to be financially beneficial in the short term. Work is underway with the detailed monitoring of temperature and humidity in each layer of the wall construction of each building. This will enable tracking in detail of the passage of vapour throughthe envelope and the identification of interstitial condensation risk and help allocate the differences to the performance of the internal vapour check and the plywood rigid air barrier. Further work will include monitoring conditions under controlled, active heating, cooling and moisture generation which should provide a stiffer test and opportunity for the structure to show a financially beneficial difference.

\section{Acknowledgments}

This research has been carried out with the support of Thomas van Raamsdonk of ProClima and the Unitec Internal Research Fund.

\section{Author Contributions}

Roger Birchmore contributed to the research design and carried out the collection and analysis of the data. Robert Tait and Andy Pivac contributed to the research design experimentation set up and conducting measurements.

\section{Conflicts of Interest}

The authors declare no conflict of interest. 


\section{References}

1. Mackintosh, L. NIWA Overview of New Zealand Climate. 2001. Available online: https://www.niwa.co.nz/education-and-training/schools/resources/climate/overview (accessed on 11 June 2014).

2. BizEE Degree Days. Weather Data for Energy Professionals. Available online: http://www.degreedays.net/\# (accessed on 22 December 2014).

3. De Groot, H. Indoor Air Quality and Health. An Analysis of the Indoor Air Quality and Health in New Zealand's Homes. 2009. Available online: http://www.archigraphic.de/_originals/pdfs/ indoor_air_quality_and_health.pdf(accessed on 11 June 2014).

4. Howden-Chapman, P.; Saville-Smith, K.; Crane, J.; Wilson, N. Risk factors for mould in housing: A national survey. Indoor Air 2005, 15, 469-476.

5. Howden-Chapman, P.; Crane, J.; Chapman, R.; Fougere, G. Improving health and energy efficiency through community-based housing interventions. Int. J. Public Health 2011, 56, 583-588.

6. New Zealand Business Council for Sustainable Development (NZBCSD). Media Release: \$20 Billion Cost of Fixing Country's Homes Less than 4\% of Their Value. 2008. Available online: http://www.scoop.co.nz/stories/BU0812/S00020.htm (accessed on 11 June 2014).

7. World Health Organisation. Guidelines for Indoor Air Quality: Dampness and Mould. 2009. Available online: http://site.ebrary.com/lib/unitech/Doc?id=10367463\&ppg=55 (accessed on 11 June 2014).

8. Sargent, S. Getting Clear on Vapour Barriers and Underlays. 2007, Build 99. Available online: http://www.buildmagazine.org.nz/articles/show/getting-clear-on-vapour-barriers-andunderlays/ (accessed on 4 February 2015).

9. Howden-Chapman, P.; Matheson, A.; Crane, J.; Viggers, H.; Cunningham, M.; Blakely, T.; Cunningham, C.; Woodward, A.; Saville-Smith, K.; O'Dea, D.; et al. Effects of insulating existing houses on health inequality: Cluster randomised study in the community. Br. Med. J. 2007, 334, 460 .

10. Easton, L.; Saville Smith, K. Homesmart Renovations - Testing Tools to Promote Sustainable Renovation. In Proceedings of the New Zealand Sustainable Building Conference, Wellington, New Zealand, 26-28 May 2010.

11. Callau, M. Upgrading Housing in NZ for Thermal Efficiency. In Proceedings of the New Zealand Sustainable Building Conference, Wellington, New Zealand, 26-28 May 2010.

12. Burgess, J.C.; Buckett, N.R.; Camilleri, M.J.T.; Burrough, L.J.; Pollard, A.R. Papakowhai Retrofit Project-Improving the Thermal Envelope and Space Heating. In Proceedings of the New Zealand Sustainable Building Conference, Wellington, New Zealand, 26-28 May 2010.

13. Su, B. Prevention of inter mould growth in housing. Archit. Sci. Rev. 2006. 49, 385-390.

14. Su, B. Indoor moisture control of Auckland houses with different ventilation systems. Int. J. Civ. Archit. Sci. Eng. 2013, 7, 411-415. 
15. Leardini, P.M.; van Raamsdonk, T. Design for Airtightness and Moisture Control in New Zealand Housing. In Proceedings of the New Zealand Sustainable Building Conference, Wellington, New Zealand, 26-28 May 2010.

16. WUFI Pro, 2D, Plus Software 2013. Fraunhofer-Institute fur Bauphysik (IBP), Holzkirchen. Available online: http://www.wufi.de/index_e.html (accessed on 21 January 2015).

17. De Groot, H.; Leardini, P.M. Indoor Air Quality and Health in New Zealand's Traditional Homes. In Proceedings of the 44th Annual Conference of the Architectural Science Association, Unitec Institute of Technology, Auckland, New Zealand, 24-26 November 2010.

18. Love, J. Mapping the Impact of Changes in Occupant Heating Behaviour on Space Heating Energy Use as a Result of UK Domestic Retrofit. Retrofit Conference, University of Salford, UK, 2012. Available online: http://www.salford.ac.uk/_data/assets/pdf_file/0005/142385/023Love.pdf (accessed on 21 January 2015).

19. Clevenger, C.M.; Haymaker, J. The Impact of Building Occupation on Energy Modelling Simulations, Joint International Conference on Computing and Decision Making in Civil and Building Engineering, Montreal, Canada, 2006. Available online: http://web.stanford.edu/ group/peec/cgi-bin/docs/people/profiles/The\%20Impact\%20of\%20the\%20Building\%20Occupant \%20On\%20Energy\%20Modeling\%20Simulations.pdf (accessed on 21 January 2015).

20. Department of Building and Housing Compliance document for New Zealand Building Code Clause H1 Energy Efficiency-Third Edition. Available online: http://www.dbh.govt.nz/ UserFiles/File/Publications/Building/Compliance-documents/H1-energy-efficiency-3rd-editionamendment-2.pdf (accessed on 21 January 2015).

21. Specification for Preservative Treatment Part 3: Plywood; SNZ 2012 AS/NZ 1604.3; Standards New Zealand: Wellington, New Zealand, 2012.

22. Plywood-Structural Part 0: Specifications; SNZ, 2012b, AS/NZS 2269.0:2012; Standards New Zealand: Wellington, New Zealand, 2012.

23. Moll, L.; van Raamsdonk, T. A New Zealand Based Study on Airtightness and Moisture Management; ProClima, NZ Ltd: Wellington, New Zealand, 2009.

24. Barley, D.; Deru, M.; Pless, S.; Orcellini, P. Procedure for Measuring and Reporting Commercial Building Energy Performance; Technical Report NREL/TP-550-38601; National Renewable Energy Laboratory, Battelle: Golden, CO, USA, 2005.

25. LASCAR Certificate of Calibration. Available online: http://www.lascarelectronics.com/ pdf-usb-datalogging/data-logger0237687001331303304.pdf (accessed on 21 January 2015).

26. Thermal Performance of Buildings-Determination of Air Permeability of Buildings-Fan Pressurization Method; European Committee for Standardization CEN/TC 89 2000. EN 13829:2000; British Standards Institution: London, UK, 2001.

27. Stoecklein, A.; Bassett, M. ALF3-A Design Tool for Energy Efficient Houses; Building Research Association of New Zealand: Judgeford, New Zealand, 1999.

28. Passive House Institute Passive House Requirements. Available online: http://www.passiv.de/en/ 02_informations/02_passive-house-requirements/02_passive-house-requirements.htm (accessed on 11 June 2014). 
29. TenWolde, A.; Pilon, C. The Effect of Indoor Humidity on Water Vapor Release in Homes. Available online: http://web.ornl.gov/sci/buildings/2012/2007\%20B10\%20papers/071_ TenWolde.pdf (accessed on 11 June 2014).

30. Mitamura, T.; Rode, C.; Schultz, J. Full-Scale Testing of Indoor Humidity and Moisture Buffering in Building Materials. In Proceedings of the Indoor Air Quality 2001, Moisture, Microbes, and Health Effects: Indoor Air Quality and Moisture in Buildings, ASHRAE: Atlanta, GA, USA, 2001. 


\title{
Modern Housing Retrofit: Assessment of Upgrade Packages to EnerPHit Standard for 1940-1960 State Houses in Auckland
}

\section{Paola Leardini and Manfredo Manfredini}

\begin{abstract}
New Zealand state housing includes a significant portion of problematic buildings constructed after the public housing scheme launched in 1936. Most of these houses are still uninsulated, thus, cold, draughty, mouldy, and progressively decaying; however, as they are fundamental elements of the country's culture, society, and environment, and are built with good quality materials and sound construction, they are suitable candidates for effective energy upgrades. This paper presents findings of a study on problems and opportunities of retrofitting the state houses built between 1940 and 1960 in the Auckland region. It advocates strategic national policies and initiatives for retrofitting, based on more challenging performance thresholds. The research defines and virtually implements an incremental intervention strategy including different retrofit packages for a typical 1950s stand-alone house. Indoor and outdoor environmental parameters were monitored over a year, and data used to establish a base case for thermal simulation. The upgrade packages were then modelled to assess their impact on the house's thermal performance, comparing heating requirements and comfort of various insulation and ventilation options. The paper reports on effective ways of preserving the integrity of such a house, while improving its thermal performance to the EnerPHit standard, and discusses the benefits of introducing this holistic approach into New Zealand retrofit practice.
\end{abstract}

Reprinted from Buildings. Cite as: Leardini, P.; Manfredini, M. Modern Housing Retrofit: Assessment of Upgrade Packages to EnerPHit Standard for 1940-1960 State Houses in Auckland. Buildings 2015, 5, 229-251.

\section{Introduction}

According to the International Energy Agency, buildings are responsible for $32 \%$ of the total final energy consumption and around $40 \%$ of the primary energy consumption in most of its member countries [1]. The growing awareness of the impact of the built environment on energy security that started with the 1973 oil crisis has made building energy efficiency a common target of many national energy conservation policies. It also has produced a wide variety of analytical, certification and rating tools aimed at assessing and enhancing the energy performance of new buildings. The effect of these measures, though, has been limited, since these instruments only partially address the activities of renovation, repair and maintenance of existing buildings, which constitute a large part of construction activity in developed countries. An increased effort towards consistent inclusion of the existing stock in policies and programmes for the improvement of energy efficiency is crucial to achieve tangible savings in building-related energy consumption and $\mathrm{CO}_{2}$ emission reduction. This is particularly relevant, as in many urban transformations retrofitting is often more effective than demolition and reconstruction in both economic and environmental 
terms as demonstrated by studies using Life Cycle Analysis [2]. Further benefits need to be accounted when the retrofit involves buildings with historic or cultural value: heritage retrofit not only contributes to the development of local culture-enhancing the quality of the urban environment while retaining its character and heritage - but also supports local economies, since this kind of retrofit requires a wide array of skilled labour on site. Overall, the conservation of existing buildings positively influences local communities, promoting resilient and sustainable urban living patterns.

In New Zealand, retrofit activity has proved to be non-cyclical (while new building construction is characterized by sharp highs and lows) [3] and has exceeded new construction, especially since the global financial crisis. According to Building Research Association New Zealand (BRANZ) already in 2008 alterations and additions (including approximately 33,000 residential consents [4]) represented about one-third the total value of new dwellings. This activity is expected to increase, due to the significant ageing of the New Zealand housing stock and the need for earthquake strengthening and other urgent maintenance interventions. Current residential buildings will constitute the largest portion of the country's housing stock in coming decades [5,6]: BRANZ estimates $85 \%$ will be still in use by 2025 [7]. However, most houses do not have sufficient insulation to provide comfortable living: about $65 \%$ were built before 1978, when mandatory insulation was first introduced with the standard NZS 4218P: 1977 Minimum Thermal Insulation Requirements for Residential Buildings. Research found that even when upgraded according to current local practice, average winter indoor air temperatures are extremely low [8], and frequently fall below the limit of $18{ }^{\circ} \mathrm{C}$ recommended by the World Health Organisation [9]. As housing is internationally recognised as a key determinant of health and the country has one of the highest incidences of asthma worldwide (with low socio-economic groups disproportionately affected [10]), the New Zealand government has committed to improve the nation's health introducing relevant policies, as well as subsidies to enhance the performance of houses, particularly those of low-income households. The state housing stock includes a significant portion of problematic houses, built in the 1940s and 1950s under the government-funded social housing scheme introduced in 1936. Most of these are managed by Housing New Zealand Corporation (HNZC), the largest public landlord in the country, which recently focused on upgrading its housing stock-especially in Auckland where $43 \%$ of state housing is concentrated [11]. Historically, due to the mild climate of this region, the extent of this problem and the urgency of actions required have been often underestimated. However, the socio-economic relevance of these interventions was confirmed by studies on the relationship between the density of state housing and area deprivation status [12] that highlight the key role of the house in improving living conditions of disadvantaged social groups [13].

This paper presents the most recent results of a study undertaken at the University of Auckland to assess the performance of pre-1978 low density state housing in the Auckland region with regard to occupant health and comfort, as well as energy efficiency, and to identify better retrofit strategies and practices. It aims to delineate a path to "nearly zero" energy housing-borrowing goals and definition of the recent European Directive 2010/31/EU (EPBD recast). The research focuses on the 1940 to 1960 housing cohort, one of the most suitable for effective renovation due to 
its pre-eminence and homogeneous composition [14]. This cohort is mainly composed of suburban detached or semi-detached houses occupied by low-income households.

This paper briefly discusses the social and cultural value of this house type. Then, it critically reviews past and current retrofit practice, highlighting the limits of its market-driven approach, and advocates future strategic national policies and initiatives for retrofitting, based on more challenging performance thresholds. The paper reports on effective ways of preserving the integrity of a typical stand-alone state house in Auckland, while improving its thermal performance to the EnerPHit Standard [15], and discusses the benefits of introducing this holistic approach into New Zealand retrofit practice.

\section{Intangible Cultural Heritage Value of Mid-Century State-Supported Housing}

Mid-twentieth century state-supported housing is an important part of New Zealand's cultural patrimony, reflecting the fundamental values, knowledge and techniques that characterise the transition of the country to the post-colonial era. These houses and settlements, with associated physical and social infrastructures, not only produced a significant portion of the country's urban fabric, but constituted the benchmark for the overall production of houses and urban extensions, both in terms of spatial patterns and technological solutions (Figure 1).

Today, they distinctively emerge in the contemporary city, since their material consistency and experiential dimensions express an approach to the construction of the living environment that substantially differs from those of antecedent and following periods. Beyond the peculiar morphological features that make these houses immediately recognisable (such as the $32^{\circ}$ pitch roof and the standard windows placed just under the soffit), what marks their difference is their consistent sets of conception, representations and practices. Their paradigm still actively engages contemporary life, to some even representing the main reference and framework for both their activity and identity [16]. The numerous projects completed with state support through direct intervention (by the Housing Division of the Ministry of Works) or financial means (through the State Advances Corporation), constitute a diffuse presence in contemporary New Zealand cities [17]. They oppose the progressive fragmentation of the current city with the integration model framed in the social welfare politic introduced in 1935 by the first Labour government. This model becomes more and more important in the current urban reorganisation towards sustainable environments for resilient communities, showing its efficacy through the vitality of its environments. Indeed, it successfully elaborates subtle articulations of fundamental integrative elements concerning the reciprocal relations between private and public spheres, urban and natural environments, senses of independence and belonging, and community and multitude. These forms of integration are testimony to the modern re-interpretation of the extraordinary effort of colonial New Zealand society to concretise the shared "happy dream of the imagination" envisioned by Edward Gibbon Wakefield in its theory of colonisation [18]. Its mid-century revision underpinned the development of a prosaic formulation of an "overall culture of family and home" [17] (p. 204); a concrete utopia deployed through a narrative structured around four key elements: family life, bucolic existence, cohesive community and diversity. On the one hand, the idea of providing the family with a retreat in the domestic idyll associated with the reconnection of urban and natural life further elaborated 
the garden suburb model derived from the British garden city that was introduced in New Zealand in 1919. It is worth noting that this model has become the desired norm of New Zealanders, orienting housing choices and preventing the development of denser urban forms, notwithstanding urban sprawl issues that have been recognised since the 1950s [19]. On the other hand, the support for individual paths of "emancipation through social identification" and the recognition of "originality in self-expression" led to the establishment of habitats of multiplicity where integration was to achieve through the manifestation of difference.

The construction of diversified domestic landscapes through the adoption of the traditional type of the detached home, confirmed as a fundamental hinge of people's life, was further developed by combining individuality with the latest functionalist precepts of rational layout and access to sun, air and nature. Coherent responses to specific environments, conceptions and practices were implemented with an idiosyncratic range of spatialisation forms that were modulated through an extremely high number of patterns, types and variations, and resulted in the creation of never repeated places and landscapes. The preliminary phase of the research presented in this paper explored the extent of this phenomenon through a quantitative analysis of the Auckland case. It showed how the high house type number (exceeding 12,000) corresponds to a very low average repetition (below five occurrences) of realisations for each type [20].

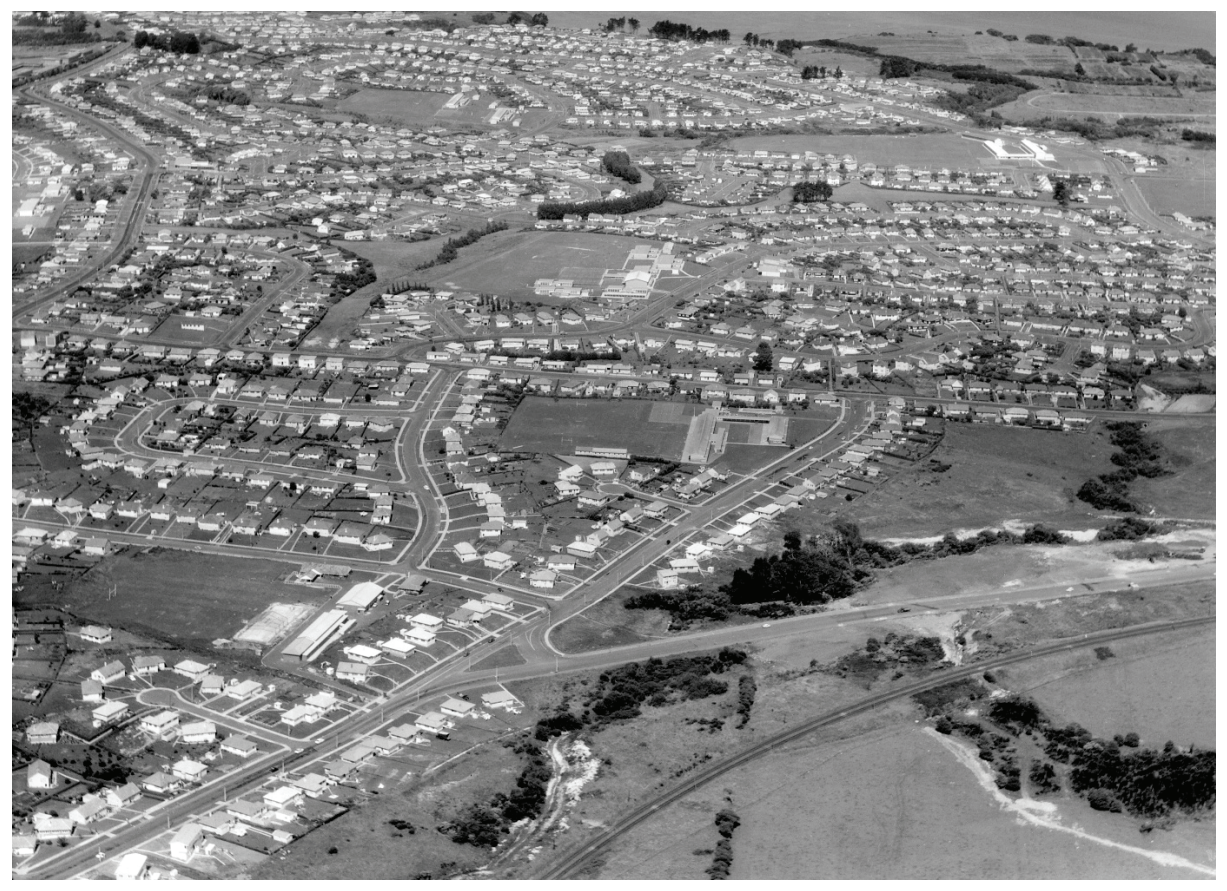

Figure 1. Glen Innes, Auckland, 12 December 1961. Whites Aviation Ltd.: Photographs. Aerial photograph of the northern part of the Tamaki state house scheme. The pilot project house is featured in the bottom left side of the image. Source: Alexander Turnbull Library, Wellington, New Zealand. Ref: WA-56411-F. http://natlib.govt.nz/records/22856887. 
The rich and diverse patrimony embedded in those domestic architectures, in families and communities of their inhabitants and in their physical and cultural landscapes, is an irreplaceable legacy of a foundational milestone of the construction of New Zealand society. As such, it cannot be preserved selectively: its multiplicity and diversity represent one of its highest achievements. It is a living representation of the great modern effort to construct a concrete and effective collective model of social, cultural and physical progress. Having been recognised by Nikolaus Pevsner as "the most ingratiating chaos one can imagine" [21], the highest challenge for the preservation of its integrity is to develop appropriate analyses and interpretations of each and every individual condition concerning people, territories and signs.

\section{New Zealand Housing Retrofit}

According to the latest OECD assessment on people's wellbeing [22], New Zealand ranks as the second highest country for housing expenses of households: on average above one quarter of disposable income. Accounting for $16.3 \%$ of total household income, the total costs of housing increased rapidly in 2013/14 [23], worsening the problem of affordability. This particularly affects Auckland, the largest city of the country and one of the five most unaffordable major markets worldwide [24]. Without reduction of the housing shortage, this situation cannot be eased, but is expected to worsen: the growing difference between demand and supply cannot be reversed, due to the limited capacity of the construction industry [25]. With this structural scarcity and a market dominated by investors - who in 2014 won more than $45 \%$ of all sales [26] - the existing housing stock is subject to intensive exploitation, resulting in an emphasis on profitability through short term capital gains. This draws attention away from running costs and building operating efficiency, further exacerbating problems arising from poor building maintenance-especially critical for those houses already unable to guarantee comfortable and healthy living conditions.

Problems affecting current New Zealand housing stock are well documented, but there is still little information available on their specific extent. The largest source of data currently accessible is a series of House Conditions Surveys conducted on representative samples by BRANZ from 1994 to 2010. They show that a large portion of the existing stock offers substandard living conditions, due to lack of maintenance: only $40 \%$ of the sample assessed in the 2010 survey, including new construction, was considered "well maintained," while $25 \%$ had defects to be urgently addressed [27]. The 2010 survey shows a high percentage of homes with some form of insulation, mainly in the roof cavity; but still, $52 \%$ of both new and old construction had no wall insulation and $45 \%$ of accessible suspended floors remained un-insulated. Lack of insulation appears to be a feature of most pre-1978 houses, despite different policies and programmes implemented within the framework of National Energy Efficiency and Conservation Strategies [28,29].

The critical condition of older houses also resulted from the limited effects on existing buildings of the New Zealand Building Code (NZBC), which includes stringent requirements for new houses insulation but offers little guidance for renovations. The provisions for energy performance and comfort of the NZBC are included in Clause H1-Energy Efficiency [30] and Clause G5-Interior Environment [31]. The latter, despite introducing a minimum internal temperature limit $\left(16^{\circ} \mathrm{C}\right)$ for old people's homes and early childhood centres, has no quantitative requirements for other 
residential buildings. Conversely, Clause $\mathrm{H} 1$ fixes the maximum building heating energy consumption by establishing the Building Performance Index (BPI), a thermal performance measure calculated as heating energy divided by the product of heating degrees total, floor area and total wall area. The recently refined BPI, though effectively requiring houses to use less heating energy than before, still allows rather low R-values for building envelopes across all New Zealand regional climates, especially inadequate in the colder areas of the country [32].

\subsection{Costs and Benefits of Housing Retrofit}

While general awareness about needs and benefits of improving living conditions has increased, it seldom translates into adequate energy retrofit actions. This is mainly because, as capital gain is the primary driver for home renovations, the emphasis on short-term financial return does not appropriately cope with long-term sustainability principles. The fact that New Zealand still enjoys some of the lowest power tariffs in the world often challenges the acceptance of future proof retrofitting plans. Page [33] found that basic efficiency measures, including ceiling and under-floor insulation, are considered worthwhile from a cost-benefit viewpoint in all locations in New Zealand; enhanced solutions though, such as wall insulation and double glazing, are not considered cost-effective when compared to the installation of heat-pumps and solid fuel burners, especially in the milder Auckland region. This approach, disregarding environmental concerns and predicted energy shortage, has supported current retrofitting practice and policies, which make basic interventions on the building envelope and heating devices, instead of addressing a comprehensive requalification of the older building stock. However, it does not consider the "non-energy benefits" of efficiency upgrade measures: comfort levels are not given as an "invariable" but included in the cost-benefit equation with the same value of energy savings. This economically driven approach has been criticised by the social scientist Philippa Howden-Chapman: focusing on positive effects of adequate house insulation on reduction of health inequality, she highlighted the relation between cold and damp houses, and mould growth, which is a trigger for respiratory illnesses [13]. Her findings showed how health costs of substandard living conditions are often paid by the weakest social groups. This study, considering predominantly low-income communities in New Zealand, concluded that retrofitting insulation in existing homes led to significantly warmer and drier living environments, which resulted in improved health and reduced days off school and work. She also proposed a cost-benefit analysis [34] that included health gains, energy and environmental savings, and suggested that the monetary value of improving housing quality is "compelling", as its total benefits equal to "one and a half to two times the magnitude of the cost of retrofitting insulation". She found that $61 \%$ of the total benefits were health sector gains, so her research confirms the social relevance of retrofitting and the need of filling the gap of research on cost-effectiveness of regulatory requirements to eventually guide their improvement.

\subsection{Retrofit Programmes}

Pre-1978 housing stock in New Zealand does not present significant typological differences between public and privately owned dwellings. Although quite varied, state houses and most 
private houses were mainly single or double-storey detached buildings, generally with small square or rectangular floor plans, with hipped or gabled roofs covered with tiles, asbestos-cement shingles or corrugated sheets. The envelope, largely timber-framed construction, was realised using a limited selection of cladding materials (timber weatherboard, brick veneer, stucco or asbestos-cement cladding) and no insulation [35]. These houses though, were very progressive compared to the bungalows and villas that preceded them. Instead of the villa layout, strictly designed in relation to the main street and disregarding cardinal directions, the new types were usually orientated to the north to allow maximum sun penetration into the main interior living areas in winter, and fitted with eaves for protection from the summer heat. Windows were larger to increase sunlight and fresh air, which were deemed very important to avoid the recent outbreak of tuberculosis and diphtheria: the window area was indeed sized to be $15 \%$ of the floor area of the room, with half of the windows openable for ventilation [36].

The innovative planning and robust construction of early state housing types, however, did not prevent them being cold and draughty: within a few years from construction, mould was discovered in $57 \%$ of the houses built from the early 1940s [37,38]. Since these and successive findings of research to investigate the correlation between insulation, ventilation, and healthy interiors did not substantiate in any housing improvement, most of those houses that have not been renovated still present the same issues. Indeed, compared to privately owned houses, the maintenance level of state-owned rental properties has often been lower for basic budget constraints, and included minimal upgrade interventions over the time.

After decades without maintenance, in 2001 the government's National Energy Efficiency and Conservation Strategy [28] set up the political and financial framework to support a major energy retrofit programme, providing a platform to extend funding for pre-1978 home insulation retrofits, including state housing stock, around the country. The programme aimed to upgrade two thirds of the stock with ceiling insulation, aluminium foil below suspended floors, hot water cylinder insulation and draught stoppers. Given the magnitude of the programme, the University of Otago was commissioned to monitor results of the upgrade process by measuring energy consumption and indoor thermal environment of a sample of 100 state houses in Dunedin [39]. Findings of this study seriously challenged the efficacy of the retrofit programme, revealing apparent limits of retrofitting to ceilings and floors only. In the South Island, this produced only small temperature increases that were insufficient to guarantee healthy indoor environments. Budget constraints have downsized public upgrade programmes. These, however, should not be evaluated in terms of simple payback times. Instead, social benefits must become the real driver, as affirmed by the successful initiative started by HNZC in the Auckland region in 2000: the Healthy Housing Programme, a comprehensive scheme aimed to reduce the risk of infectious diseases related to overcrowding. Beside health and social interventions to identify families with health needs and provide social services accordingly, the scheme included housing retrofitting intervention to address cold, mould and ventilation issues [40].

In 2009, the Energy Efficiency and Conservation Authority made a further attempt to overcome the poor energy performance of a still significant part of the housing stock, launching the Warm Up New Zealand: Heat Smart Programme. Its scope was to subsidise costs of retrofitting insulation 
and installation of clean heating for houses built before 2000. Although this programme proved beneficial, in particular to health [41], the subsidies are not subject to the achievement of any specific performance level (under the assumption that any amount of insulation installed represents an appreciable improvement), so its efficacy is difficult to assess and its results are questionable. Furthermore, it has been observed that long-term effects can be challenged, considering that basic retrofit interventions may reduce future energy savings, i.e., "the potential revenues of an energy efficiency investment [as] future amendments to improve quality are very improbable because they will not pay back, thus impeding future sustainable development" [42]. This highlights the need for a more holistic retrofitting approach, addressing wall insulation, airtightness, and ventilation.

\subsection{From Common Practice to EnerPHit Standard}

In recent years, the growing market pressure due to housing shortage and speculative investments in the real estate sector have made old houses in need of renovation a valuable target. In this context, interventions are usually market-driven and mainly concentrated on spatial layout improvements, with bathroom and kitchen upgrades being the most effective way to achieve short-term paybacks; there is very little concern for thermal comfort, indoor air quality and airtightness. Building consents must be lodged only when the improvements require substantial alterations of the existing envelope, and for new additions, which must comply with the criteria and energy performance requirements of the Code. Underfloor and roof insulation retrofits are exempt from building consent according to Schedule 1 of the New Zealand Building Act. However, retrofitting insulation into a wall cavity either requires a building consent or specific approval from the authority, as the impact on the existing building can be potentially problematic (e.g., for moisture accumulation, timber framing and cladding durability, fire and electrical safety). Despite that, thermal resistance of retrofitted walls does not need to comply with the Building Code Clause H1-Energy Efficiency [30]. This set of rules accepts that the partial and cosmetic retrofit interventions mentioned above are executed without considering housing performance and indoor comfort matters [43].

Even the most forward-thinking examples of retrofitting simply aim at improving the energy performance of buildings to match minimum requirements of the current NZBC, which do not guarantee acceptable indoor air quality and comfort. Indeed, concerning current Code requirements, a recent study undertaken at the University of Auckland [44] focusing on New Zealand houses built after 2000, showed that thermal comfort in Code-compliant houses is generally below the acceptable threshold defined by international standards [45]. Measured air-leakage rates appeared high compared to both European houses and international guidelines, and yet the resulting indoor air quality was relatively poor. This is also consequent to the fact that neither the NZBC nor the New Zealand ventilation standards set any airtightness target for residential buildings that rely on natural ventilation. NZBC Clause H1-Energy Efficiency states that the airtightness of the building envelope has to be taken into account, but does not define any specific threshold. Clause G4-Ventilation [46] only requires openable areas (window and doors) to be more than 5\% of the floor area for air quality and summer cooling, relying on the New Zealand tradition of satisfying ventilation needs with background infiltration, i.e., uncontrolled access of outdoor air into the 
building through leaks in the envelope. Without any Code requirement, retrofitting practice is not compelled to address airtightness, especially given the additional cost of achieving acceptable performance using conventional construction methods. However, this exposes existing houses to new risks, as shown by recent research on the effects of retrofitting insulation according to common practice (i.e., without a ventilated air cavity or a vapour control layer), which confirmed the possibility of formation of interstitial condensation and related moisture problems [47]. This not only suggests that the Building Code is in need of more detailed identification of benchmarks to promote healthy and energy efficient houses, but also that retrofitting practice must set higher goals to be effective.

The depicted scenario of retrofitting practice demands a new set of upgrade strategies aiming to more ambitious performance targets. Based on energy efficiency and comfort, the performance-based Passive House Standard [48] seems to offer effective answers to the identified issues. Developed in Germany in the early 1990s by Professors Bo Adamson and Wolfgang Feist, this is the fastest growing energy performance standard in the world, with over 30,000 buildings realised to date. Its strengths lie in the simple, non-prescriptive approach, based on the excellent thermal performance and airtightness of the building envelope. According to the Passivhaus Institut (PHI), " $a$ Passivhaus is a building for which thermal comfort can be achieved solely by post-heating or post-cooling of the fresh air mass, which is required to achieve sufficient indoor air quality conditions - without the need for additional recirculation of air" [49]. A traditional heating system is no longer essential, due to reduced heating needs. The other important feature is its excellent indoor air quality, achieved by reducing the air infiltration rates and supplying filtered fresh air that can be post heated by a mechanical ventilation unit with heat recovery (MVHR).

The standard was first officially introduced to New Zealand in 2011, with the launch of Passive House Institute New Zealand (PHINZ) and the construction of the first Passive House in Auckland - certified the following year. Since then Passive Houses have spread throughout the country, especially in the North Island. In the retrofitting sector, however, the standard has been widely disregarded, due to its presumed high costs. Indeed, it can be technically and economically difficult to achieve the requirements of the standard in old residential buildings, due to unavoidable thermal bridges, constraints with insulation installation and air-leakage issues. Considering these aspects, in 2010 PHI introduced the EnerPHit standard for energy retrofits with a certification method based on listed Passive House components. These however, are available for cool-temperate climates only. To overcome this limitation, PHI started a research process to define international component requirements that can serve as the basis for international EnerPHit criteria [50]. The requirements are the result of an economic optimization process applied to a simplified set of seven climate zones around the globe.

PHI also developed an alternative certification method for building retrofit based on performance requirements. These are looser than those for new construction, with maximum space heating and cooling demand of $25 \mathrm{kWh} /\left(\mathrm{m}^{2} \mathrm{a}\right)$ and a limit value of the envelope air permeability rate $n_{50}<1.0 \mathrm{~h}^{-1}$ - that is expressed as the ratio between the air leakage rate at 50 Pascal air pressure and the total volume of air in cubic metres. While the airtightness limit will remain valid for all climate zones of the future international EnerPHit standard, heating and cooling demand will be 
tuned to the different zones: from $30-35 \mathrm{kWh} /\left(\mathrm{m}^{2} \mathrm{a}\right)$ in cold and artic climates to $15-20 \mathrm{kWh} /\left(\mathrm{m}^{2} \mathrm{a}\right)$ in warm and warm-temperate climates.

\section{Research Methodology}

This paper presents the most recent findings of a long-term study carried out at the University of Auckland on eco-retrofitting of pre-1978 state housing in Auckland. This study proposes and tests a new holistic approach to improve upon the sheer economic cost-benefit path driving the majority of common retrofit practices, which disregard social costs (in particular the ones related to health and environment issues highlighted above) and socio-cultural matters. Consistent with sustainable development principles and aiming to preserve the social, cultural and material integrity of the mid-twentieth century housing stock in New Zealand, the proposed approach sets specific criteria for its upgrade. It draws upon a comprehensive investigation of social, environmental and architectural aspects, as well as local and international retrofitting best practices. On the basis of the successful implementation in New Zealand of the Passive House standard for new construction, in the Auckland region, the research proposes to adopt the EnerPHit energy efficiency and comfort targets for retrofitting in warm-temperate climates. The applicability of the standard is tested through the development of a pilot project that includes an intervention strategy articulated on four incremental phases.

Overall, the research included the following consecutive phases: (1) Collection, systematization and analysis of documents and information, and subsequent definition of a type stock pool of pre-1978 low density state houses; (2) Selection and analysis of representative case-studies in Auckland; (3) Comparative evaluation and adaptation to New Zealand of internationally recognized diagnostic and measurement protocols, and building performance standards; (4) Identification and documentation of a pilot project and two control cases including measured drawings, stratigraphic and 3D models, diagnostic survey (building pathologies, Blower Door test, thermography, stratigraphic inspection and analysis of structural and functional inadequacies) and occupants' interviews; (5) Environmental parameters' measurement campaign of the pilot project and the two control cases; (6) Development of an intervention strategy and related retrofit packages with assessment through thermal simulation (comparing annual heating requirements of various insulation and ventilation options) for a pilot-project in Auckland.

While the results of phases $1-5$ have already been published [20,51], this paper focuses on the last research phase concerning the definition and evaluation of the intervention strategy and its phased incremental retrofit packages.

\subsection{Pilot Project and Measurement Campaign}

The pilot project concerns a representative three-bedroom state house. It was built according to one of the most common types (6/1163) developed by the Department of Housing Construction in 1940s, as part of the large Tamaki state house scheme in the outer Auckland suburb of Glen Innes (Figure 2). It is a single story building of $93 \mathrm{~m}^{2}$ on sloping ground with a west-oriented living room. Although this house passed into private ownership, it has retained the original layout and 
features. The typical timber frame construction, enclosed by weatherboard cladding and internal plasterboard lining, has a tiled roof and a suspended (carpeted) timber floor. It sits on perimeter concrete walls that define a basement space partially accessible due to the sloping terrain (Figure 3). Originally without insulation, a few years ago the house was refurbished with the addition of polyester blankets over the ceiling and under the floor, between joists. Lying in a prominent position that benefits from good solar access, it has a well-ventilated basement space, which makes it very dry in comparison to the majority of similar residential buildings of the neighbourhood (Figure 4).

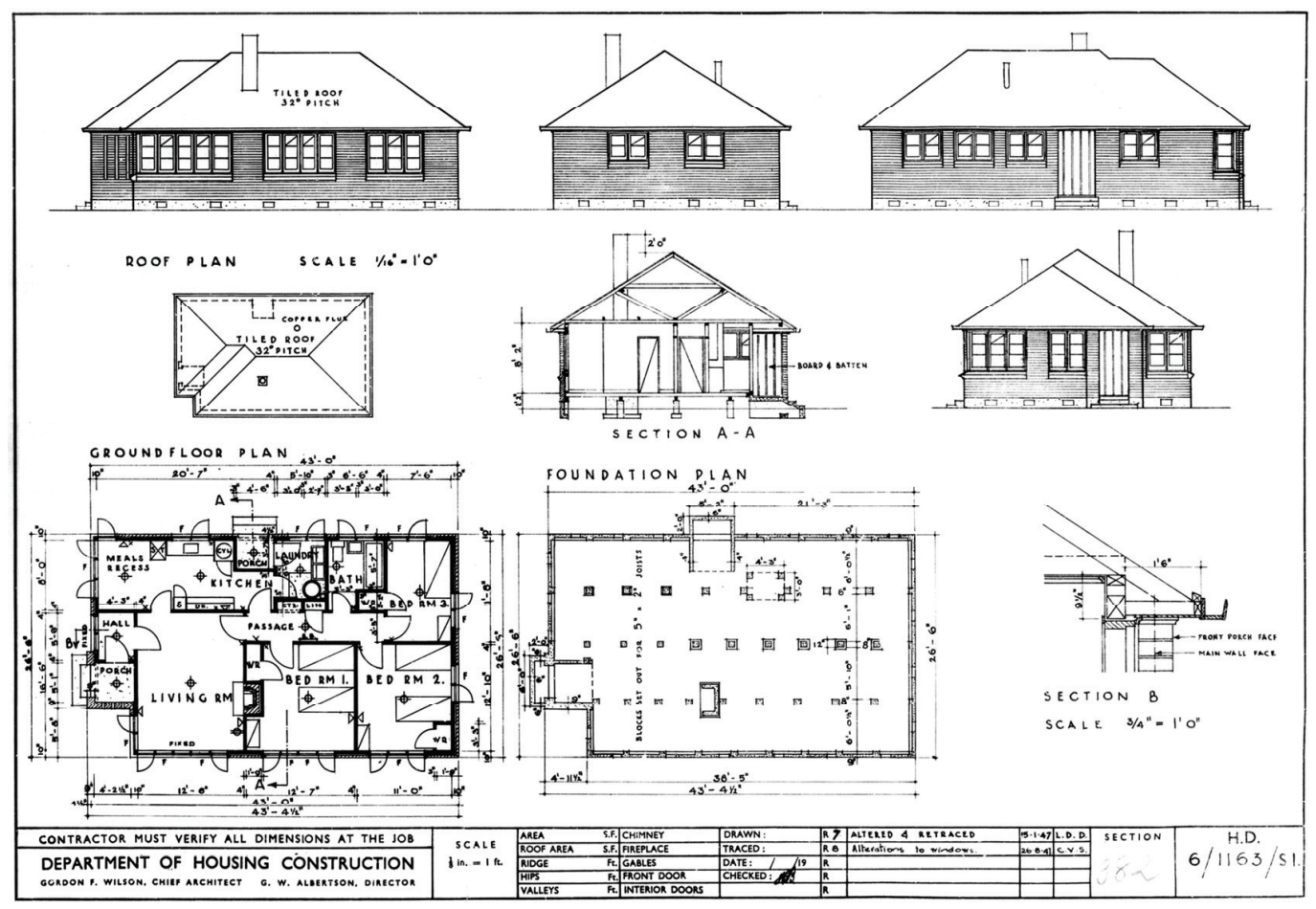

Figure 2. Original documentation of the Department of Housing Construction for the house type 6/1163 of 1947 . The house selected for the pilot project is its wooden variant for sloping ground. Ref: Source: Housing New Zealand Auckland Regional Office, Floor plans and details for standard houses, naval housing, pre-cuts, demonstration flats, star flats and point flats. Ref: Q12_47, BBKD 10559 Box1 (Archives New Zealand/Te Rua Mahara o te Kāwanatanga, Auckland Regional Office). 


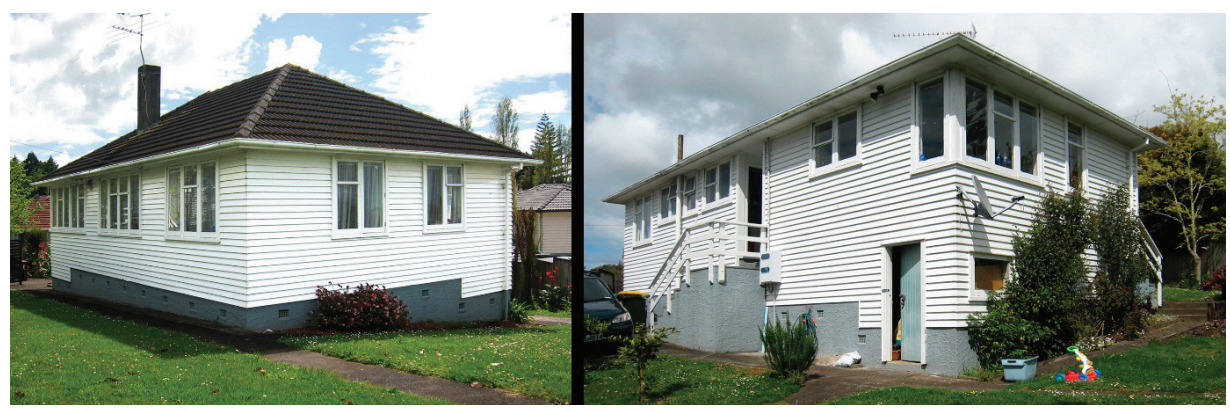

Figure 3. South-west (street side) and north-east (backyard side) views of the pilot project house in 2012 .

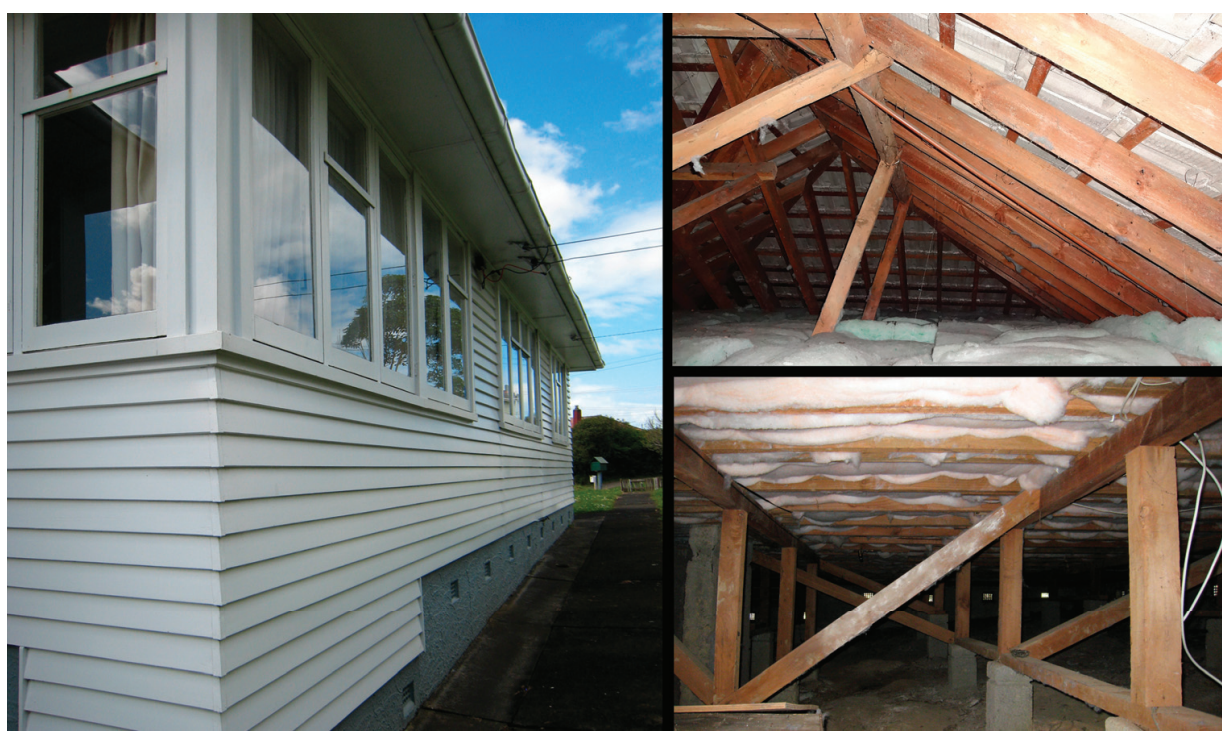

Figure 4. North-west facades, hipped roof cavity (timber rafters with concrete tiles and polyester insulation) and basement cavity (jack studs, sleepers and bracing struts on concrete blocks supporting floor joists with polyester insulation in-between) of the pilot project house in 2012 .

In 2012, a measurement campaign of pre-retrofitting indoor and outdoor environmental parameters was carried out to provide empirical data. It was used to validate the simulation model of the various energy upgrade packages and provide a reliable basis to the quantification of potential energy savings and indoor comfort improvements. It included one year of continuous data collection (from January to December) of indoor and outdoor environmental parameters using data loggers (iButton and $\mathrm{HOBO}$ ) for measuring air temperature, relative humidity and $\mathrm{CO}_{2}$, and energy metering systems (smart meters). Weather conditions (air temperature, relative humidity, wind speed and direction, and global solar radiation) were recorded using hourly data provided by the National Institute of Water and Atmospheric Research. 


\subsection{Intervention Strategy}

The strategy adopted to achieve the defined primary social and cultural objectives of the research proposes the conservation and sustainable development of peoples' habitats with particular regard to the integrity of the existing buildings and their physical environment. It includes the definition of a set of actions, framed in participatory processes both in the planning and implementation phases, to allow residents to remain in place, consolidating their communities and sense of ownership (only strictly necessary short-term relocations are foreseen). These actions aim to preserve the material patrimony and aesthetic character of the houses, including their overall building structure, spatial organisation and decorative framework.

Economic concerns have also been fundamental drivers of the strategy and its implementation through incremental retrofit packages, particularly aiming to provide flexible investment options and minimise costs of components, material, procurement and labour. The proposed phasing comprises a four-step series of coherent, effective, discrete and independent retrofit packages, respectively concerning the improvement of thermal performance and airtightness of ceiling (PH-S1), flooring (PH-S2), external walls (PH-S3), as well as the installation of new windows and mechanical heat-recovery ventilation system (PH-S4). All employed building components and construction materials are cost-effective and already available in the New Zealand market. Construction and installation techniques are simple, rapid (mainly add-on non-structural interventions) and common practice in this country.

To further sustain the cost-effectiveness and practicality of the proposed model, the packages are designed either to not require building consent (PH-S1 and $\mathrm{PH}-\mathrm{S} 2$ ) or to ease the consenting process (PH-S3 and $\mathrm{PH}-\mathrm{S} 4)$ through the use of standardised components, details and installation methods that may be complemented with pre-compiled documentation. This is of particular relevance in New Zealand as it is aligned with the principles behind the peculiar regulatory framework that, for the consenting process, includes owner-builder (DIYers) exemptions from the 2004 introduced Licensed Building Practitioners Scheme [52].

\subsection{Thermal Modelling and Calculation with Passive House Planning Package}

The retrofit packages are designed to achieve progressive improvements of the envelope's thermal performance and airtightness up to the EnerPHit standard for warm-temperate climates (heating demand $=20 \mathrm{kWh} /\left(\mathrm{m}^{2} \mathrm{a}\right)$ and air permeability rate $\left.\mathrm{n}_{50}<1.0 \mathrm{~h}^{-1}\right)$. For thermal simulation, a "base case" was defined to represent the condition of the house as originally built, without insulation. Four cumulative upgrade levels were then modelled, each including all the interventions of the levels before it. In addition the house was modelled for the current situation (i.e., with the insulation Basic Package, thus labelled SH-BP) and to comply with the current Building Code (SH-NZBC). Simulation models were validated using data from environmental parameter measurements. Air infiltration levels were estimated according to previous studies undertaken by BRANZ [53,54] and to match EnerPHit requirements. To meet the standard, mechanical ventilation with heat recovery (MHRV) was introduced only in the final package (PH-S4). 
Preliminary estimates of heat losses and space heating demands were made for the various upgrade options using the computer thermal simulation program Virtual Environment [51]. This helped verify the technical and economic feasibility of the proposed interventions, and the magnitude of associated energy savings. On this basis, in the next phase of the study, refined retrofit packages were tested using the Passive House Planning Package (PHPP), the Excel spreadsheet-based design tool specifically developed by PHI to assist architects and designers plan and verify Passive Houses towards certification. It calculates building components' U-values, heating, cooling and primary energy demand, ventilation rates for comfort as well as the risk of overheating in the warmer season. Furthermore, it compiles climate data from many locations worldwide, including Auckland and other main centres in New Zealand. Validated with dynamic simulation tools as well as with measured data, the PHPP energy balance module has proven to be surprisingly precise, calculating the energy balances of a building to an accuracy of $\pm 0.5 \mathrm{kWh}$.

The specific tool embedded in PHPP for calculation of $U$-values allowed a more detailed exploration of individual building component assemblies, taking into account specific construction details and locally available products. To achieve the thermal performance required by the EnerPHit standard (PH-S4), the following insulation options were selected: $190 \mathrm{~mm}$ polyester insulation in the attic space between and above ceiling joists $\left(R\right.$-value $\left.=4.55 \mathrm{~m}^{2} \mathrm{~K} / \mathrm{W}\right) ; 125 \mathrm{~mm}$ glasswool insulation under the floor between joists and $45 \mathrm{~mm}$ EPS between bearers $\left(R\right.$-value $\left.=4.18 \mathrm{~m}^{2} \mathrm{~K} / \mathrm{W}\right) ; 100 \mathrm{~mm}$ glasswool insulation between studs plus $45 \mathrm{~mm}$ of the same material between battens in the service cavity $\left(R\right.$-value $\left.=3.20 \mathrm{~m}^{2} \mathrm{~K} / \mathrm{W}\right)$; low-e, double-glazing timber windows filled with argon gas $\left(R\right.$-value $\left.=0.73 \mathrm{~m}^{2} \mathrm{~K} / \mathrm{W}\right)($ Table 1$)$.

Table 1. Upgrade levels, including a summary of $R$-values $\left(\mathrm{m}^{2} \mathrm{~K} / \mathrm{W}\right)$ used for each element of the building fabric for PHPP calculation.

\begin{tabular}{|c|c|c|c|c|c|c|}
\hline Upgrade & Insulation & $\begin{array}{l}\text { Ceiling } \\
\mathbf{m}^{2} \mathbf{K} / \mathbf{W}\end{array}$ & $\begin{array}{c}\text { Floor } \\
\mathbf{m}^{2} \mathbf{K} / \mathbf{W} \\
\end{array}$ & $\begin{array}{c}\text { Walls } \\
\mathbf{m}^{2} \mathbf{K} / \mathbf{W}\end{array}$ & $\begin{array}{l}\text { Window } \\
\mathbf{m}^{2} \mathbf{K} / \mathbf{W}\end{array}$ & $\begin{array}{c}\text { Airtightness } \\
\left(n_{50}\right) h^{-1}\end{array}$ \\
\hline Base Case & House as built (no insulation) & 0.27 & 0.74 & 0.60 & 0.22 & 20 \\
\hline PH-S1 & $\begin{array}{l}\text { Ceiling insulation } \\
100+90 \mathrm{~mm} \text { polyester between and above joists }\end{array}$ & 4.55 & 0.74 & 0.60 & 0.22 & 20 \\
\hline PH-S2 & $\begin{array}{l}\text { As PH-S1 + Floor insulation } \\
125 \mathrm{~mm} \text { glasswool between joists }+40 \text { EPS } \\
\text { between bearers }\end{array}$ & 4.55 & 4.18 & 0.60 & 0.22 & 20 \\
\hline PH-S3 & $\begin{array}{l}\text { As PH-S2 + Wall insulation } \\
145 \mathrm{~mm} \text { glasswool between studs and battens }\end{array}$ & 4.55 & 4.18 & 3.20 & 0.22 & 6.5 \\
\hline PH-S4 & $\begin{array}{l}\text { As PH-S3 + Windows upgrade and airtightness } \\
\text { low-e, argon filled double glazing timber windows }\end{array}$ & 4.55 & 4.18 & 3.20 & 0.73 & 1 \\
\hline SH-BP & $\begin{array}{l}\text { SH insulated with basic package (as-it-is) } \\
50 \mathrm{~mm} \text { polyester under floor }+150 \mathrm{~mm} \text { above ceiling }\end{array}$ & 2.42 & 1.73 & 0.60 & 0.22 & 20 \\
\hline SH-NZBC & House to comply with NZBC & 2.90 & 1.30 & 1.90 & 0.26 & 6.5 \\
\hline
\end{tabular}

For this study the new PHPP 9 was used, which allows the direct comparison of different variants, together with their economic evaluation. Additional retrofit options were then tested to verify the specific impact of airtightness, mechanical versus natural ventilation and window 
performance. The aim of this investigation was to assess under what insulation, ventilation and airtightness conditions the EnerPHit requirements could be met, thus, exploring alternative and affordable solutions to overcome constraints imposed by the existing construction.

\section{Results and Discussion}

Overall results of PHPP calculation confirm the impact on energy savings of different insulation (ceiling, floor, walls and windows) and airtightness improvements that was found with VE thermal modelling - assuming constant indoor thermal comfort conditions associated with air temperature equal to $20^{\circ} \mathrm{C}$. However, the heating demand of the various options tested is higher, due to the different nature of the two tools and slightly different boundaries conditions (e.g., climate data, treated floor area and ventilation schedule). Furthermore, PHPP provides more detailed calculations of heat losses, including the effect of thermal bridges, natural ventilation and solar gains according to surrounding shading elements.

Figure 5 shows specific annual heating demand $\left[\mathrm{kWh} /\left(\mathrm{m}^{2} \mathrm{a}\right)\right]$ and overheating risk [\% of time] for each of the upgrade scenarios calculated with PHPP. The graph illustrates how improvements from the uninsulated house (Base Case) to one that complies with NZBC reduce heating demand by $74 \%$. This result however can be used as a reference only, because old, draughty houses are not usually evenly heated to reach comfortable indoor temperatures-while new Code-compliant houses should be. A more significant result is that, by improving insulation of ceiling, floor and external walls in the path to meet the EnerPHit standard (PH-S3) [50], annual heating demand drops below that of the Code-compliant house SH-NZBC (78 versus $81.4 \mathrm{kWh} /\left(\mathrm{m}^{2} \mathrm{a}\right)$ ) - using for calculation the same airtightness $\left(n_{50}\right)$ of $6.5 \mathrm{~h}^{-1}$, the average value for new construction according to BRANZ [54].

The successive retrofit package PH-S4, with additional interventions to achieve the EnerPHit standard, is essential for reducing heating demand, enhancing indoor comfort and moisture control. New windows $\left(R\right.$-value $\left.=0.73 \mathrm{~m}^{2} \mathrm{~K} / \mathrm{W}\right)$ and overall air permeability rate $n_{50}$ equal to $1.0 \mathrm{~h}^{-1}$, reduce heating demand further, to one quarter (from 78 to $19.5 \mathrm{kWh} /\left(\mathrm{m}^{2} \mathrm{a}\right)$ ), while assuring a constant comfortable indoor temperature of $20{ }^{\circ} \mathrm{C}$. Compared to the Code-compliant house (SH-NZBC), this package produces a marginal increase in overheating risk (due to improved airtightness and reduced heat losses through the envelope), although still below the $10 \%$ limit. The residual discomfort issues could be easily avoided by employing more effective window shading (such as external shutters or blinds) or selective glazing.

Other relevant results emerge from the evaluation of the initial packages' implementation: the first step (PH-S1) confirms the importance of ceiling insulation not only in terms of energy savings (with 38\% heat demand reduction) but also for reducing the risk of overheating. The additional floor insulation in step 2 (PH-S2) has a lower impact on heating demand (15\% reduction) and, decreasing heat loss through the floor in summer, slightly increases the overheating risk. The amount of insulation installed under the floor is excessive for achieving comfortable surface temperatures, but is indispensable to attain the envelope's performance required to meet the standard, as it compensates for the limited amount of insulation that can be accommodated in the wall construction. 


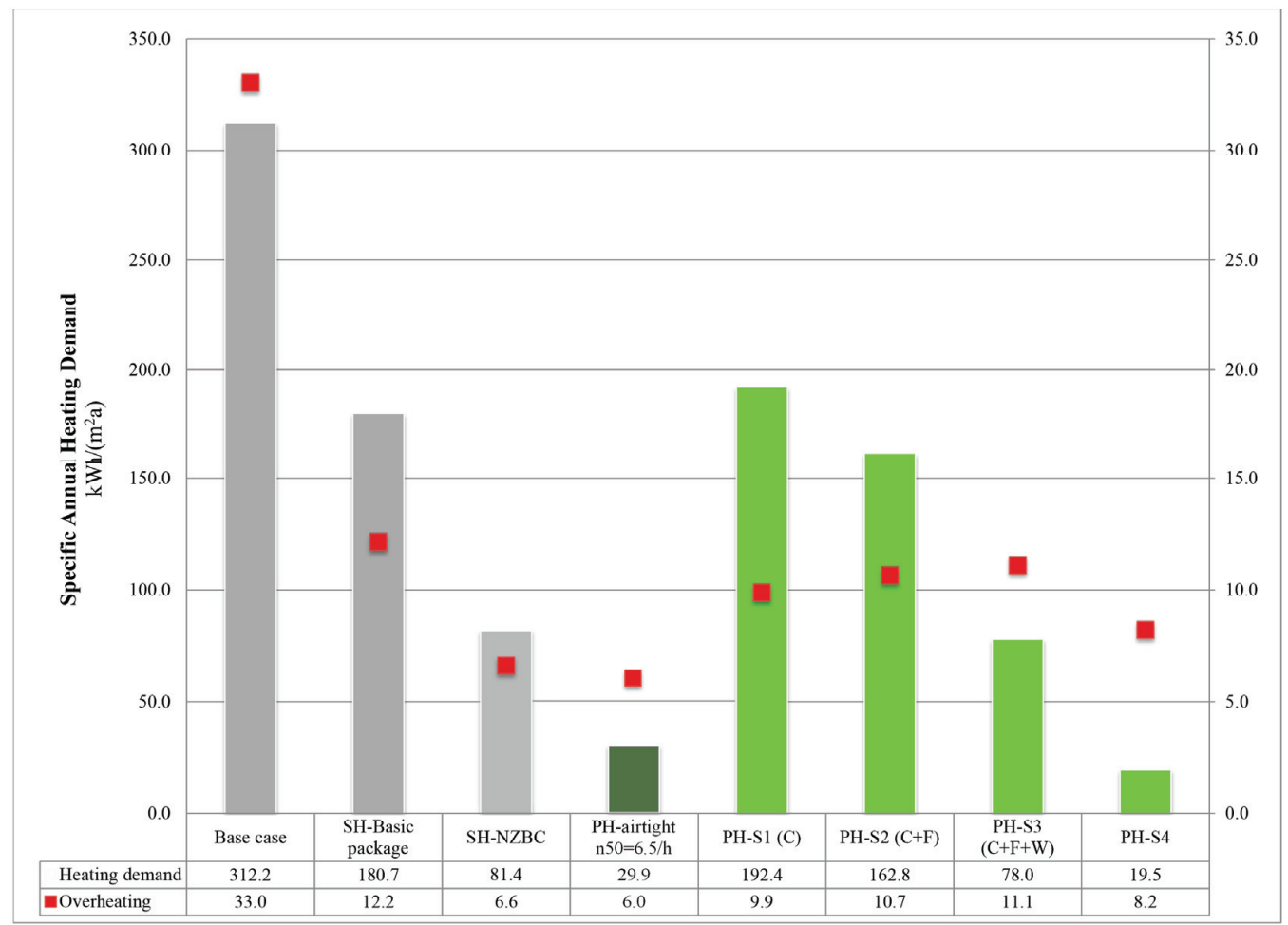

Figure 5. Comparison of specific annual heating demands $\left(\mathrm{kWh} /\left(\mathrm{m}^{2} \mathrm{a}\right)\right)$ and overheating time $(\%)$ of the base case and different retrofitting packages.

Overall, results also prove the positive impact on heating demand reduction of two factors often neglected by the common retrofit practice in New Zealand: wall insulation and air infiltration. This becomes evident through the analysis of outward and inward flows (losses and gains) through the envelope, shown in Figures 6 and 7, which provide details of benefits from each intervention. The comparison of the heat flow break-down of the existing house (SH-BP) and that meeting the NZBC requirements (SH-NZBC) shows a significant reduction of heating demand due to new windows, improved airtightness ( $n_{50}$ drops from 20 to $6.5 \mathrm{~h}^{-1}$ ) and wall insulation; the latter proves to have the greatest impact (54\% of the reduction).

U-values input in the calculation can be achieved using different building components and mechanical ventilation solutions available in New Zealand, taking into consideration technologies compatible with the country's construction practice; results show that the heating demand requirements of the EnerPHit standard can theoretically be achieved. However, given the extremely low energy consumption involved, performance could be affected by thermal bridging and airtightness issues. Further research is required to develop thermal bridge-free construction details and effective solutions for a continuous airtight envelope. Indeed, while insulation can easily be installed in existing houses to guarantee the necessary thermal performance of the envelope, achieving levels of airtightness required by the EnerPHit standard can be challenging, due to 
construction and preservation issues. Deep retrofit interventions, including floor and ceiling replacement as well as new internal partitions, offer the best opportunity to realise a continuous airtight layer inside the timber structure of the existing envelope. Airtight layers (oriented strand board, plywood boards or vapour control membranes) can be installed in the flooring between joists and finishing, in the external wall between the existing timber structure and the new service cavity (to be added inside for accommodating additional insulation and reducing thermal bridges), and in the ceiling below the existing joists. All joints of floor, wall and ceiling airtight layers must be tightly sealed. Achieving the required airtightness levels can be challenging in retrofit interventions where existing floor, ceiling, internal load bearing walls and partitions are maintained in place, as the additional airtight layer added on top of existing floor, wall and ceiling surfaces is interrupted by the structure of each internal partition, and so is difficult to seal. Airtightness though, is key to reducing heating demand, as PHPP calculations show that, in the final package (PH-S4) shifting $n_{50}$ from $1 \mathrm{~h}^{-1}$ (EnerPHit limit) to $8 \mathrm{~h}^{-1}$ (value estimated for contemporary retrofitted Auckland houses), results in $60 \%$ heating demand increase. As shown in Figure 7, even increasing $n_{50}$ just to $6.5 \mathrm{~h}^{-1}(\mathrm{PH}-\mathrm{S} 4 *)$, which is the same airtightness of SH-NZBC, increases specific ventilation losses from 5.1 to $19.7 \mathrm{kWh} /\left(\mathrm{m}^{2} \mathrm{a}\right)$ - higher than the specific annual heating demand of $\mathrm{PH}-\mathrm{S} 4$ - and heating demand to $29.9 \mathrm{kWh} /\left(\mathrm{m}^{2} \mathrm{a}\right)$.

Experimental research is required to evaluate the achievability of the desired infiltration rate in either intervention (also considering cost issues) since there are no examples in New Zealand of retrofitted houses to EnerPHit standard. This should also include studies of technological and economic feasibility to identify the achievable airtight levels and, if lower than required by the standard, to evaluate how to compensate for the increased heating demand with either additional insulation or higher performance windows (without compromising indoor comfort and building integrity).

The comparative analysis of different ventilation systems showed the effect of MHRV on heat demand and summer comfort. The impact of airtightness and ventilation is evident when comparing the heat flow breakdown of PH-S3 and PH-S4 (Figure 6), where the installation of MHRV and new double-glazed timber windows halves ventilation losses. In winter, a balanced MHRV proves to be key to maintaining heating demand below the limit of $20 \mathrm{kWh} /\left(\mathrm{m}^{2} \mathrm{a}\right)$, as with natural ventilation it would increase from 19.5 to $28.1 \mathrm{kWh} /\left(\mathrm{m}^{2} \mathrm{a}\right)$. Summer overheating was identified as a potential issue for the existing house, which has its largest windows exposed to the western afternoon sun. PHPP calculations show that heat recovery only provides a small reduction of the overheating percentage. Thus, overall energy consumption could be reduced (and the New Zealanders' propensity for indoor/outdoor flow favoured) by coupling winter use of the balanced heat recovery ventilation system with increased (when compared to the actual schedule provided by the occupants) summer natural ventilation. Increasing natural ventilation in summer only reduces the percentage of overheating during the warmest months, given the minimal air temperature difference between inside and outside, but is insufficient for night cooling of lightweight building structures. To achieve better indoor comfort conditions, a suitable option would be to utilise mechanical ventilation in summer (activated by temperature or humidity sensors) without heat recovery. 


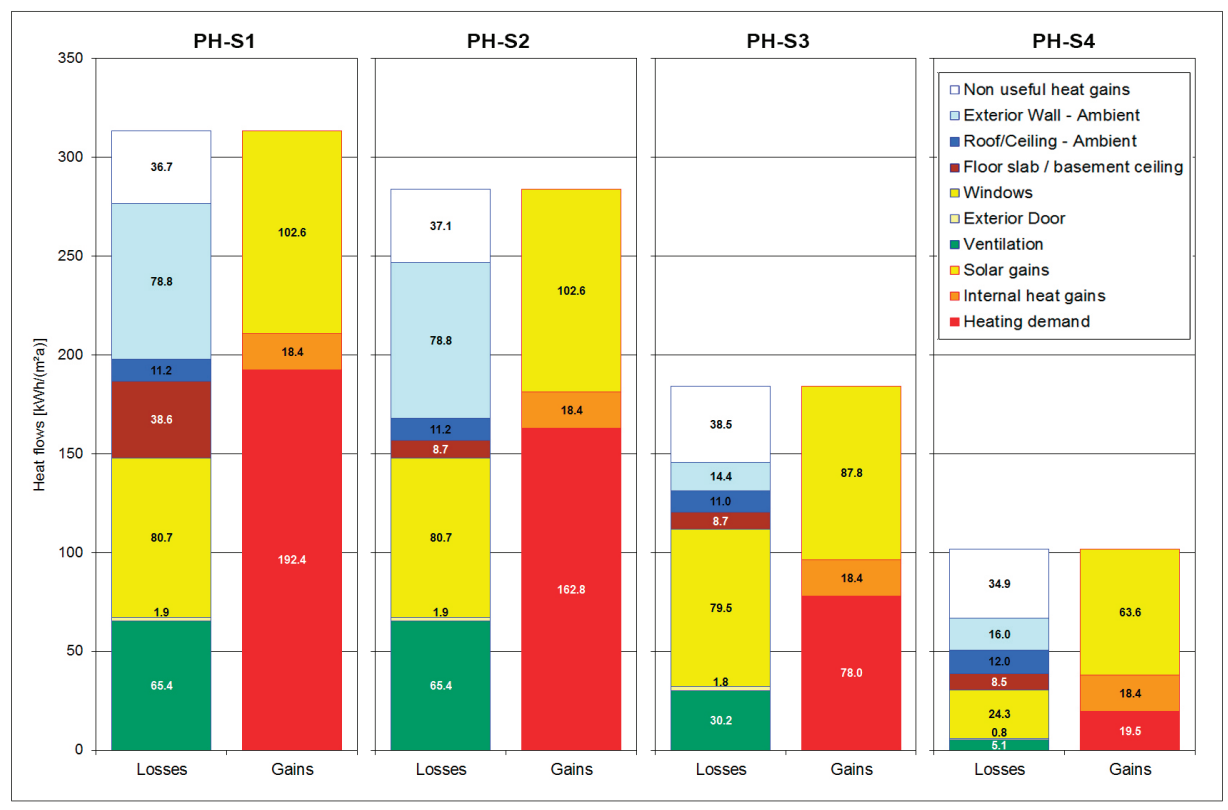

Figure 6. Comparison of heat flows (losses and gains) of the four incremental retrofit packages to meet the EnerPHit standard calculated with PHPP.

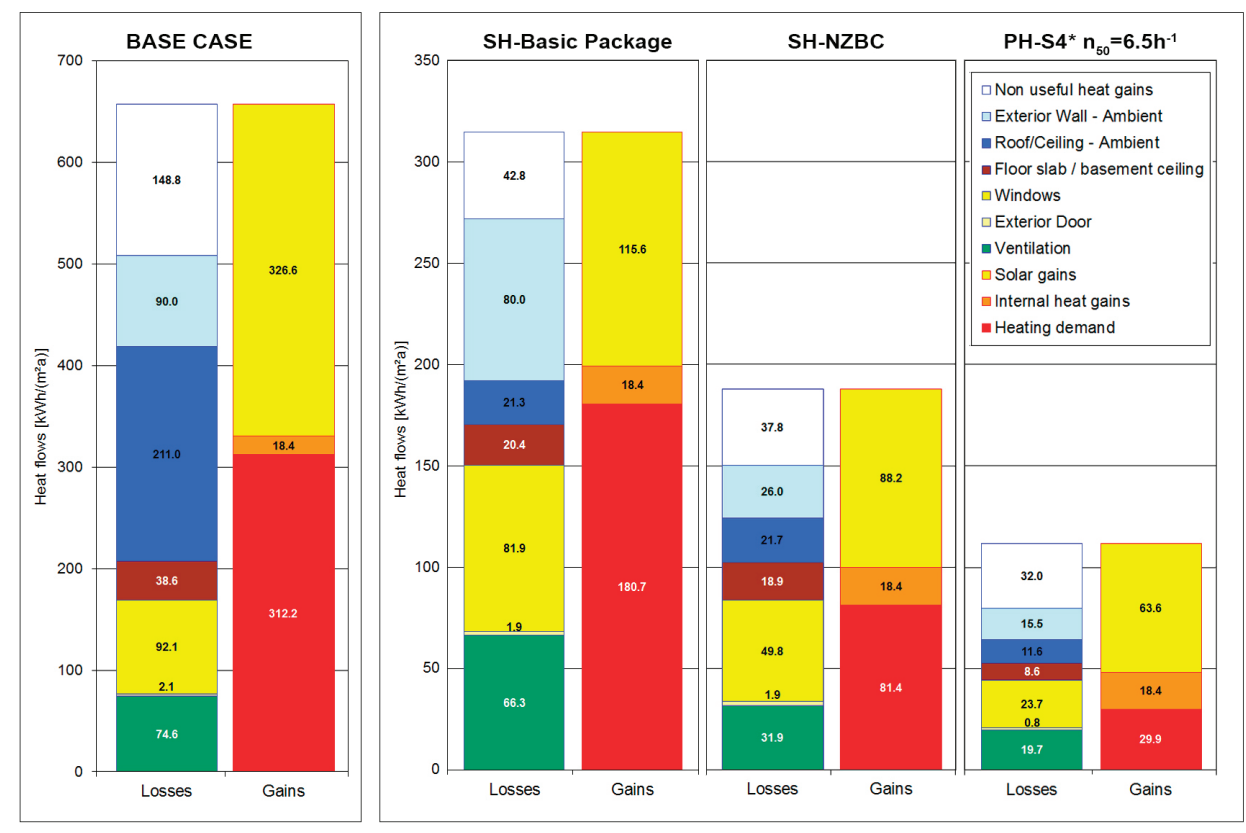

Figure 7. Heat flows (losses and gains) of the Base Case (axis maximum value: $700 \mathrm{kWh} /\left(\mathrm{m}^{2} \mathrm{a}\right)$ ) and comparison of heat flows (losses and gains) of SH-BP, SH-NZBC and $\mathrm{PH}-\mathrm{S} 4 *$ (axis maximum value: $350 \mathrm{kWh} /\left(\mathrm{m}^{2} \mathrm{a}\right)$ ) calculated with PHPP. 
Costing the upgrades and undertaking a cost-benefit analysis of the investments over a long-term period would enable a full assessment to be made for the upgrade of the national stock to EnerPHit standard. The complete retrofit intervention in the cost-benefit analysis should include a fully insulated and airtight envelope, high performance windows with external shadings to prevent overheating in summer, and supplemental space heating (normally distributed through the low-volume MHRV system). As 1940-1960s standalone houses represent an important portion of the national housing stock, results of this analysis can be extrapolated to different climate zones to estimate levels of insulation required and associated costs. Previous research [55] has provided some indicative costing for upgrading state houses to NZBC requirements in different climate zones: as Passive Houses require extra levels of insulation, airtightness and higher performance windows, this initial estimate needs to be increased. Design optimization/standardization and product availability on the New Zealand market are possible ways to overcome expected barriers to retrofitting to EnerPhit standard.

\section{Conclusions}

The research discussed in this paper challenges the current New Zealand retrofit practice that, driven mainly by financial return, disregards social, cultural and environmental matters lying beyond regulatory prescriptions and engenders severe problems of health, energy waste and building decay. These issues are of primary importance when dealing with pre-1978 state housing, still largely occupied by the most economically vulnerable part of the population.

In consideration of the World Health Organisation [9] suggestion that, for long-term solutions, financial support to households for fuel expenses needs to be subordinated to housing energy upgrade, this study argues that subsidies should be streamed towards achieving economically feasible nearly zero-energy buildings, to detach living conditions from the risks presented by the use of unreliable heating sources and exacerbation of financial constraints. This goal, for the existing Auckland building stock, can be achieved through retrofitting interventions that meet the EnerPHit standard.

This study uses a holistic retrofit approach to develop a retrofitting strategy based on incremental interventions that are defined, prioritised, evaluated and optimised, using a representative case-study; a 1950s state house located in Auckland. The use of thermal modelling and PHPP calculation allows assessing the impact of different factors - such as wall insulation, ventilation mode, and air infiltration — on the building energy performance.

The assessment of a basic retrofit package — only including ceiling and floor insulation (SH-BP and $\mathrm{PH}-\mathrm{S} 2$ ) - shows that the common interventions supported by governmental subsidies are not sufficient to effectively reduce energy consumption. Through the comparative evaluation of the results of different retrofit interventions modelled with the PHPP software, the study illustrates how effective results can be achieved with comprehensive retrofit interventions that combine insulation and airtightness of the whole building envelope with controlled ventilation.

Detailed calculations with PHPP show that, given the mild climate of the region, the specific insulation requirements of the EnerPHit standard can be achieved using existing technology and construction materials available on the local market. However, airtightness represents the main 
challenge and further empirical research is needed to develop technologically and economically viable retrofit details for a thermal bridge-free and airtight envelope.

Overall, the approach adopted in this study is also consistent with the on-going research project EuroPHit [56], which aims to establish a certification scheme for stepwise retrofit with Passive House components. In cases of step-by-step deep retrofit, a comprehensive renovation plan, covering both present and future steps, can help ensure the integrity of the building throughout the whole process. This scheme though, and the European EnerPHit standard, were developed for application in countries with cool-temperate climates. International criteria for Passive House components and the associated EnerPHit certification, currently under development, are likely to make certification in New Zealand more challenging by decreasing heating demand limits according to warmer climates. However, as the retrofit packages proposed in the study were developed by selecting the most cost effective components, there seems to be scope for further improvements of envelope performance in order to match future requirements.

Lastly, it is important to remark that New Zealand state housing retrofit is case-specific, despite the standardised nature of these buildings, and evolutive, since the achievement of high performance-based standards, such as EnerPHit, benefits from the steady improvement of construction technology. This will be taken into account in the future phases of this research, which will shift from theory and calculation to practice: with the implementation of the identified retrofit strategy, this study aims to prove that this approach to retrofitting is not only technically feasible and economically viable, but also socially and environmentally desirable, thus encouraging essential changes in New Zealand practice.

\section{Acknowledgments}

The authors acknowledge the financial support from the University of Auckland. They also acknowledge the support of Housing New Zealand Corporation. Special thanks go to Passivhaus Institut for granting access to their databases, as well as to unpublished documents and tools.

\section{Author Contributions}

The co-authors both contributed equally to this research project and the writing and review of this article.

\section{Conflicts of Interest}

The authors declare no conflict of interest.

\section{References}

1. International Energy Agency. Available online: https:/www.iea.org/aboutus/faqs/ energyefficiency/ (accessed on 28 April 2014).

2. Preservation Green Lab. The Greenest Building: Quantifying the Environmental Value of Building Reuse; National Trust for Historic Preservation: Seattle, WA, USA, 2011. 
3. Poot, J. Building activity brings many benefits for NZ. Build Magazine 2011, 125, 30-31.

4. Page, I.C.; Fung, J. Housing Life Cycle and Sustainability. BRANZ Study Report 214; Building Research Association New Zealand: Porirua, New Zealand, 2008.

5. Department of Building and Housing. New Zealand Housing Report 2009/2010: Structure, Pressures and Issues; Department of Building and Housing: Wellington, New Zealand, 2010.

6. Auckland Council. The Draft Auckland Plan; Auckland Council: Auckland, New Zealand, 2011.

7. Building Research Association New Zealand. Building a Better New Zealand. Annual Review 2014; Building Research Association New Zealand: Porirua, New Zealand, 2014.

8. Isaacs, N.; Camilleri, M.; French, L.; Pollard, A.; Saville-Smith, K.; Fraser, R.; Rossouw, P.; Jowett, J. Energy Use in New Zealand Households: Report on the Year 10 Analysis for the Household Energy End-use Project (HEEP). BRANZ Study Report 155; Building Research Association New Zealand: Porirua, New Zealand, 2006.

9. World Health Organization. Regional Office for Europe. Housing, Energy and Thermal Comfort; World Health Organization: Copenhagen, Denmark, 2007.

10. Pattemore, P.K.; Ellison-Loschmann, L.; Asher, M.I.; Barry, D.M.J.; Clayton, T.O.; Crane, J.; D’Souza, W.J.; Ellwood, P.; Ford, R.P.K.; Mackay, R.J.; et al. Asthma prevalence in European, Maori, and Pacific children in New Zealand: ISAAC study. PediatrPulmonol 2004, 37, 433-442.

11. Regional Growth Forum. Auckland Regional Affordable Housing Strategy; Auckland Regional Council: Auckland, New Zealand, 2003.

12. Olssen, A.; McDonald, H.; Grimes, A.; Stillman, S. A State Housing Database: 1993-2009; Motu Working Paper; Motu Economic and Public Policy Research: Wellington, New Zealand, 2010.

13. Howden-Chapman, P.L.; Carroll, P. Housing \& Health: Research, Policy and Innovation; Steele Roberts: Wellington, New Zealand, 2004.

14. Page, I.; Ryan, V. It Takes All Types: A Typology of New Zealand Housing Stock Retrofits. In Innovation and Transformation, Proceeding of the SB10 Conference, Wellington, New Zealand, 26-28 May 2010.

15. Passive House Institute. EnerPHit: Certification Criteria for Retrofits; Passive House Institute: Darmstadt, Germany, 2012.

16. Schrader, B. We Call It Home: A History of State Housing in New Zealand; Reed: Auckland, New Zealand, 2005.

17. Ferguson, G. Building the New Zealand Dream; Dunmore: Palmerston North, New Zealand, 1994.

18. Wakefield, E.G. A View of the Art of Colonization, with Present Reference to the British Empire; J. Parker: London, UK, 1849.

19. Holden, H.C. Summary; Department of Internal Affairs: Wellington, New Zealand, 1959.

20. Manfredini, M.; Leardini, P. Eco-Retrofitting Analysis, Strategies and Pilot Project for State Housing in Auckland, New Zealand. In Proceedings of the Retrofit 2012 Academic Conference, Manchester, UK, 24-26 January 2012. 
21. Pevsner, N. The Ingratiating Chaos. In Pevsner on Art and Architecture: The Radio Talks; Games, S., Ed.; Methuen: London, UK, 2002; pp. 259-264.

22. Organisation for Economic Co-Operation and Development (OECD). How's Life? 2013: Measuring Well-Being; OECD Publishing: Paris, France, 2013.

23. Statistics New Zealand. Household Economic Survey (Income): Year Ended June 2014. Available online: http://www.stats.govt.nz/browse_for_stats/people_and_communities/ Households/HouseholdEconomicSurvey_HOTPYeJun14/Commentary.aspx\#housing (accessed on 10 December 2014).

24. Demographia. 10th Annual, Demographia International Housing Affordability Survey: 2014. Ratings for Metropolitan Markets; Demographia: Belleville, IL, USA, 2013.

25. Building Research Association New Zealand. Auckland Housing Forecasts and the Unitary Plan; Building Research Association New Zealand: Porirua, New Zealand, 2013.

26. Ingerson, J. Where and What Are Investors Buying? Westpac REDnews, 6 October 2014. Available online: http://www.westpac.co.nz/rednews/property/where-and-what-are-investorsbuying/ (accessed on 10 December 2014).

27. Buckett, N.R.; Marston, N.J.; Saville-Smith, K.; Jowett, J.H.; Jones, M.S. BRANZ Study Report SR 240-Preliminary BRANZ 2010 House Condition Survey Report-Second Edition; Building Research Association New Zealand: Porirua, New Zealand, 2011.

28. Energy Efficiency and Conservation Authority and Ministry of the Environment. National Energy Efficiency and Conservation Strategy. Towards a Sustainable Energy Future; Energy Efficiency and Conservation Authority and Ministry of the Environment: Wellington, New Zealand, 2001.

29. New Zealand Government. National Energy Efficiency and Conservation Strategy. Making it Happen; New Zealand Government: Wellington, New Zealand, 2007.

30. Department of Building and Housing. Compliance Document for New Zealand Building Code Clause H1 Energy Efficiency, 3rd ed.; New Zealand Government: Wellington, New Zealand, 2011.

31. Department of Building and Housing. Compliance Document for New Zealand Building Code Clause G5 Interior Environment; New Zealand Government: Wellington, New Zealand, 2011.

32. McChesney, I.; Cox-Smith, I.; Amitrano, L. Thermal Insulation in New Zealand Homes: A Status Report; Beacon Pathway Limited: Auckland, New Zealand, 2008; pp. 52-53.

33. Page, I. Cost Benefits of Housing Retrofits. In Proceeding of the SB10 Conference Innovation and Transformation, Wellington, New Zealand, 26-28 May 2010.

34. Howden-Chapman, P.L.; Chapman, R.; Viggers, H.; O’Dea, D.; Kennedy, M. Retrofitting houses with insulation: A cost-benefit analysis of a randomized community trial. J. Epidemiol. Community Health 2009, 63, 271-277.

35. Elkink, A. Renovate 1940-1960s; Building Research Association New Zealand: Porirua, New Zealand, 2011.

36. Firth, C. State Housing in New Zealand; Ministry of Work: Wellington, New Zealand, 1949.

37. Brien, R.M.; Winsome, D.R. Investigation into Causes and Control of Moulds in State Houses; Department of Scientific and Industrial Research: Wellington, New Zealand, 1944. 
38. Marsden, E. State House-Erection at Dominion Physical Laboratory; Department of Scientific and Industrial Research: Wellington, New Zealand, 1946.

39. Lloyd, C.R. Fuel poverty in New Zealand. Soc. Policy J. N. Z. 2006, 27, 142-155.

40. Clinton, J.; Mahony, F.; Irvine, R.; Bullen, C.; Kearns, R. The Healthy Housing Programme: Report of the Outcomes Evaluation; Housing New Zealand Corporation: Wellington, New Zealand, 2006.

41. Grimes, A.; Denne, T.; Howden-Chapman, P.; Arnold, R.; Telfar-Barnard, L.; Preval, N.; Young, C. Cost Benefit Analysis of the Warm Up New Zealand: Heat Smart Programme; Ministry of Economic Development: Wellington, New Zealand, 2012.

42. Ebel, W.; Feist, W.; Kaufmann, B. Economy and Financing of Efficiency: New Buildings, Renovation and Step by Step Renovation. In Proceedings of the 18th International Passive House Conference 2014, Aachen, Germany, 25-26 April 2014; Passive House Institute: Darmstadt, Germany, 2014; pp. 145-154.

43. Guidance on Building Code Compliance for Retrofitting Insulation in External Walls. Available online: http:/www.dbh.govt.nz/retrofitting-insulation-guidance\#fid20 (accessed on 10 December 2014).

44. Leardini, P.; Rosemeier, K.; Ong, A. Ventilation's Pivotal Role for Indoor Air Quality of Houses in New Zealand. In Proceedings of the Healthy Buildings 2012, Brisbane, Australia, 8-12 July 2012.

45. American Society of Heating, Refrigerating, and Air-Conditioning Engineers. ASHRAE Standard 55-2010: Thermal Environmental Conditions for Human Occupancy; American Society of Heating, Refrigerating, and Air-Conditioning Engineers: Atlanta, GA, USA, 2010.

46. Ministry of Business, Innovation and Employment. Acceptable Solutions and Verification Methods for New Zealand Building Code Clause G4 Ventilation, 3rd ed.; New Zealand Government: Wellington, New Zealand, 2014.

47. Leardini, P.; de Groot, H. Indoor Air Quality and Health in New Zealand's Traditional Homes. In Proceedings of the 44th Annual Conference of the Australian and NZ Architectural Science Association, Auckland, New Zealand, 24-26 November 2010.

48. Passive House Institute. Certified Passive House-Certification Criteria for Residential Passive House Buildings; Passive House Institute: Darmstadt, Germany, 2013.

49. The Passive House-Definition. Available online: http://passipedia.passiv.de/passipedia_en/ basics/the_passive_house_-_definition (accessed on 14 April 2014).

50. Bastian, Z. International EnerPHit Certification Criteria for Energy Retrofit with Passive House Components. In Proceedings of the 18th International Passive House Conference 2014, Aachen, Germany, 25-26 April 2014; Passive House Institute: Darmstadt, Germany, 2014; pp. 615-624.

51. Leardini, P.M.; Manfredini, M.; Callau, M. Energy Upgrade to Passive House Standard for Historic Public Housing in New Zealand. In Proceedings of the 49th AICARR International Conference, Rome, Italy, 26-28 February 2014.

52. The Building Act 2004; Brookers: Wellington, New Zealand, 2004. 
53. Bassett, M.R. Building Site Measurements for Predicting Air Infiltration Rates. In ASTM Special Technical Publication 904; ASTM: Philadelphia, PA, USA, 1986; pp. 365-383.

54. McNeil, S.; Quaglia, L.; Bassett, M.; Cunningham, M.; Overton, G.; Plagmann, M.; Boulic, M.; Leardini, P.; Rosemeier, K.; Smith, I. Trends in Airtightness of New Zealand Homes. In Proceedings of the 19th International Congress of Biometeorology Conference, Auckland, New Zealand, 5-9 December 2011.

55. Lloyd, C.R.; Callau, M.F.; Bishop, T.; Smith, I.J. The efficacy of an energy efficient upgrade program in New Zealand. Energy and Buildings 2008, 40, 1228-1239.

56. EuroPHit. Available online: http://europhit.eu/project (accessed on 10 December 2014). 


\title{
Measuring the Weathertight Performance of Flashings
}

\author{
Mark Bassett and Greg Overton
}

\begin{abstract}
Residential buildings are now better engineered to manage rainwater following the leaking building problem in New Zealand. The next challenge is to improve the weathertightness of medium-rise buildings which often use joint details widely applied on low-rise buildings but are subject to higher wind pressures and surface runoff rates. This study begins to address this challenge by measuring the water leakage performance limits of the following common flashings with static and dynamic rain and wind loads to see how their performance might be improved: (a) Horizontal $\mathrm{H}$ and $\mathrm{Z}$ jointers between direct fixed sheet claddings; (b) The window head flashing in a cavity wall; (c) A horizontal apron flashing at the junction between a roof and wall. At this stage, water penetration resistances have been measured but the data has not yet been discussed in the context of wind pressures and rain loads on mid-rise buildings. All of the joints were found to resist water leakage to pressures equivalent to the hydrostatic head of the upstand, so long as there were no air leakage paths through the joint. When vents were added, or openings were present that might arise due to construction tolerances, then the onset pressure for leakage was found to fall by as much as $50 \%$. Vents, of course, are essential for ventilation drying in rainscreen walls and even with vents present, the onset of leakage was at generally at least twice the $50 \mathrm{~Pa}$ wet wall test pressure applied in New Zealand. Opportunities were found to improve the way vented joints deal with runoff by enlarging the gap between the cladding and flashing. This prevented the outer joint volume from filling with water and occluding the vents. The apron flashing was found to cope better than a window head joint with runoff, because of the larger $35 \mathrm{~mm}$ vertical gap between the cladding and apron.
\end{abstract}

Reprinted from Buildings. Cite as: Bassett, M.; Overton, G. Measuring the Weathertight Performance of Flashings. Buildings 2015, 5, 130-148.

\section{Introduction}

Most water leaks in residential buildings occur at joints between claddings and components such as windows, etc. This is illustrated in Figure 1 using data from a survey of leaking buildings in New Zealand by Bassett et al. [1]. Over $60 \%$ of leakage sites were at junctions between claddings and other components and less than $40 \%$ were associated with roof and wall claddings. Contributing causes were found to include the omission of traditional metal flashings at junctions between components along with limited protection from eaves in newer building designs. A similar fraction " $26 \%$ " of water entry points around window and door junctions with claddings were identified by Morrison Hershfield Ltd. [2] in a survey of leaking buildings in British Columbia, Canada. In that survey, $90 \%$ of water leakage sites were found to be at junctions between materials and components or at penetrations through the cladding. 


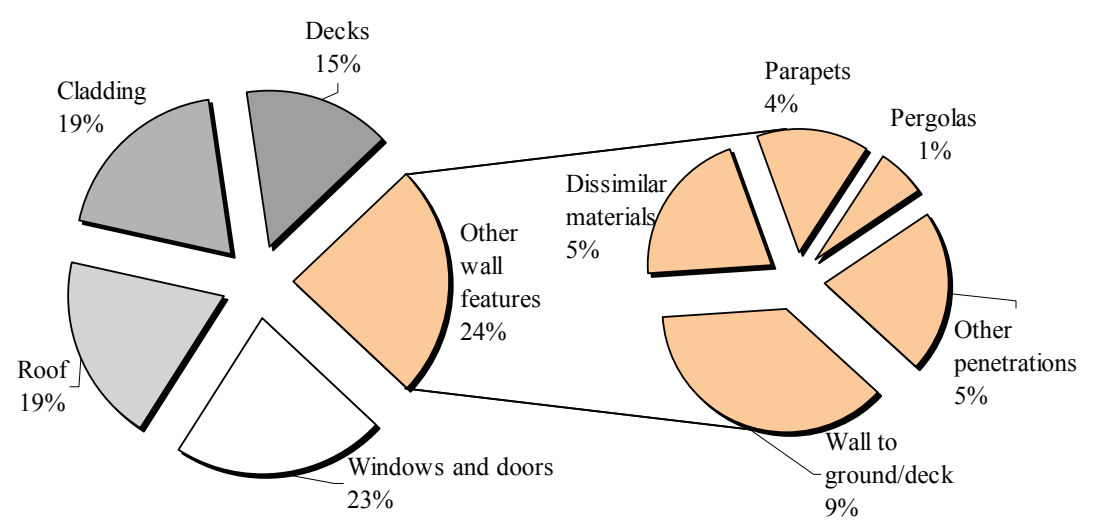

Figure 1. Water entry sites in New Zealand leaking buildings.

The Department of Building and Housing (now part of the Ministry of Business, Innovation and Employment) responded to the leaking building problem by modifying the compliance document, E2/AS1 External Moisture, of the New Zealand Building Code (NZBC) to improve the standard of water management in wall and roof designs (Department of Building and Housing [3]). Most of the changes were based on the 4Ds (deflection, drainage, drying and durability) approach developed in Canada by Hazleden and Morris [4] and incorporated a water-managed cavity behind the cladding. Additional important changes include flashings around the perimeter of windows and doors.

A new test procedure E2/VM1 "Verification Method" (Department of Building and Housing [5]) was also developed to apply the following tests and criteria to claddings and flashings on cavity walls:

- Water should not systematically reach the dry side of the cavity during any of the tests (including above the upstand on horizontal flashings above cavity closers);

- The first test in E2/VM1 is a static and cyclic pressure test of the complete wall system;

- The second test introduces defects in the cladding around flashings to confirm that water is successfully controlled by drainage paths including the back of the cladding and flashings at junctions between components or different claddings;

- Finally, the "wetwall test" applies 50 Pa pressure difference across the cladding and checks that water leaks through the cladding are confined to the drainage paths and do not bridge the cavity.

These tests apply the following criteria to flashings in cavity walls:

- The flashing forms part of the drainage path for water on the back of the cladding;

- The flashing must rainscreen the joint and prevent water from reaching the underlay with a rain intensity of at least $3 \mathrm{~L} / \mathrm{m}^{2} \cdot \min$ and a $50 \mathrm{~Pa}$ air pressure difference between the wetwall and the cavity.

Requiring the wall underlay to remain dry during testing is quite stringent and specific to New Zealand. It is the primary reason for this investigation of flashing upstand heights in claddings. There are many questions about the performance of flashings that are not answered by the E2/VM1 procedure and which this research is starting to address: 
- How far should the flashing upstand extend upwards behind the cladding to deal with wind driven rain?

- Do the upstand heights of flashings need to be adjusted to cope with rain bouncing of a roof-particularly important for apron flashings between roof and wall?

- Do cavity closers have a role in managing air and water entry through the joint?

- How should cladding to flashing clearances be sized to control runoff?

- Do hems folded into metal flashings improve the weathertight performance of flashings?

Perhaps the most pressing need for research on the leakage performance of joints is in support of timber framed walls above the height limit for E2/AS1 (three stories maximum but nominally $10 \mathrm{~m}$ above ground). Many of these buildings adapt E2/AS1 claddings and flashings to timber framed infill panels that are exposed to higher wind pressures and surface runoff rates than low rise residential buildings.

There are few scientific investigations of the water leakage performance of joints and how their design might be improved to cope with extreme exposure. One of the earliest investigations (Ishikawa [6]) measured the leakage characteristics of joints in a metal curtain wall and concluded that the key elements were a large external opening to prevent a water film from bridging the gap, and an airtight internal joint to support wind pressures. Further studies by Herbert [7] and [8] measured water entry rates through labyrinth type joints exposed to driving rain in field trials, showing that the important attributes of successful joints were an airtight inner wall and sloped drainage to deal with water entering the joint. A more recent study (Lacasse et al. [9]) measured leakage rates through specific defects in walls such as missing lengths of sealant, and used the leakage function of wind pressure and rain load to estimate the moisture loads that have to be managed by vapour diffusion and ventilation drying within the wall. Water leakage rates were measured as a function of runoff rates and static wind pressure and fitted to an empirical relationship that was then used to estimate moisture entry loads in a range of North American climates. A similar approach to developing water leakage functions for joints is followed in this study but the next step of comparing leakage rates with the capacity of joints to control leakage with drainage and ventilation drying is outside the scope of this paper. A future study will follow the procedures developed by Sahal and Lacasse [10] and determine the rain loads, wind pressures and runoff rate for claddings for taller buildings in New Zealand.

\section{Measured Rain Leakage Characteristics}

Equipment illustrated in Figure 2 was used to measure the water leakage characteristics of joints between building components. It consists of a pressure chamber connected to a fan and a fluctuating piston that together apply a steady pressure and a superimposed fluctuating pressure across the specimen. The pressure amplitude can be changed by adjusting the piston stroke although below $0.2 \mathrm{~Hz}$ there was simply not enough travel to reach large pressure amplitudes. In all of the fluctuating pressure tests described in this paper, the pressure amplitude was 2:1 as required in E2/AS1. The wall specimen measured 0.7 by $0.7 \mathrm{~m}$ (area $0.49 \mathrm{~m}^{2}$ ) and the length of joint under investigation was $0.53 \mathrm{~m}$. 


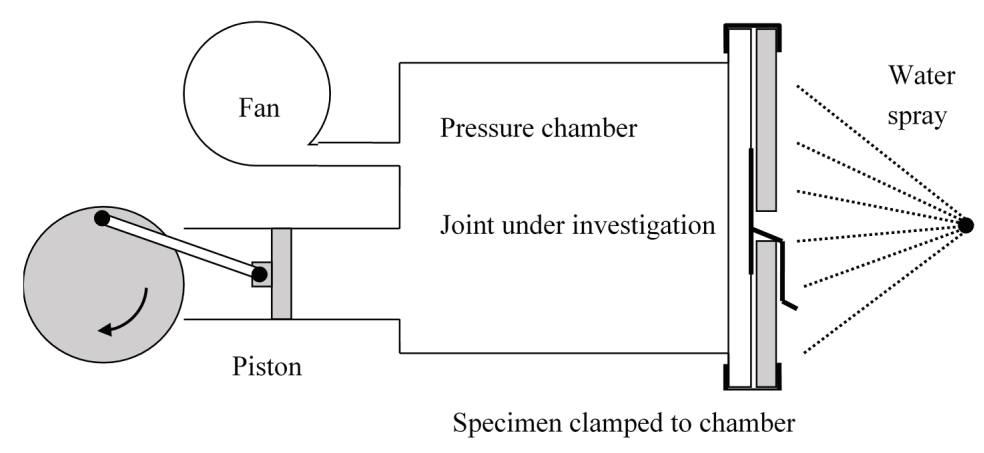

Figure 2. Equipment for measuring the weathertightness characteristics of joints.

A range of water spray nozzles were used to wet the sample area with between $3.4 \mathrm{~L} / \mathrm{m}^{2} \cdot \min$ (corresponding to the minimum rain load called for in E2/VM1) and $0.08 \mathrm{~L} / \mathrm{m}^{2} \cdot \mathrm{min}$. This is similar to the rain loads applied to large wall assemblies $\left(0.02-3 \mathrm{~L} / \mathrm{m}^{2} \cdot \mathrm{min}\right)$ by Bassett et al. [11] and provides a wide enough range of rain intensity to develop rain leakage functions that include most rain events. In these measurements the runoff rate was found to be a more useful measure of water delivered to the joint.

The leakage rates measured through junctions using this equipment establish performance limits for joints rather than actual leakage rates that might apply in mid-rise buildings in New Zealand. In a later project will use these leakage characteristics to propose flashing upstands dimensions for these buildings.

\section{Typical Flashed Joints Seen in Residential Buildings}

There are a large number of joints described in E2/AS1 but the three junctions illustrated in Figure 3 capture most of the essential features of flashings and their application in walls with cavities or direct fixed claddings.

The three joints chosen for what is a preliminary study are:

- A Z jointer between direct fixed plywoodwall panels;

- A window head flashing in a cavity wall;

- An apron flashing between a roof and a higher section of cavity wall.

Upstand heights in these joints have evolved from field experience although recent changes to extend the applicability of E2/AS1 to an "extra high" wind zone were responsible for precautionary increases to upstand heights in walls with cavities and direct fixed claddings -35 to $60 \mathrm{~mm}$ for window head flashings and 75 to $90 \mathrm{~mm}$ for roof to wall apron flashings. Another recent change required mandatory hems to the top of flashing upstands used in extra high wind zones. In lesser wind zones, the hem can be traded for an additional $25 \mathrm{~mm}$ of upstand height. These were largely precautionary changes ahead of applicable field experience or laboratory results of the type that this study aims to provide. Another significant change that came with more widely adopting cavity construction was the provision for vents in a cavity closer. Vents are potential air leakage paths and 
an entry point for air carried spray. This project set out to link cladding overhang and clearances in joints with the effectiveness with which the joint rainscreens against water entry.

\section{Cladding is direct fixed}

\section{Horizontal joint in plywood cladding}
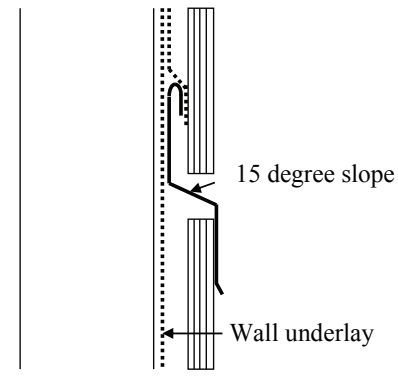

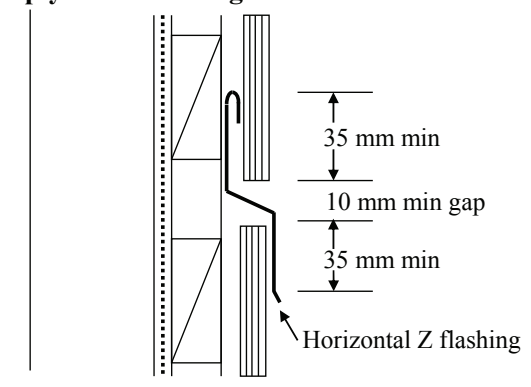

Window head with fiber-cement cladding
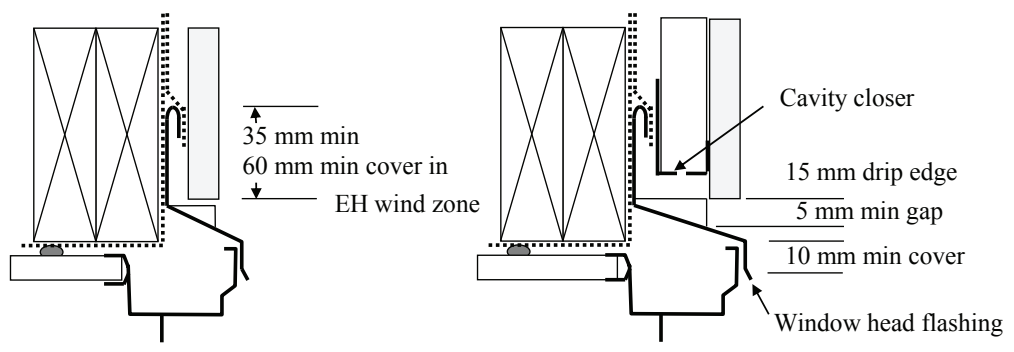

Apron flashings to metal roof
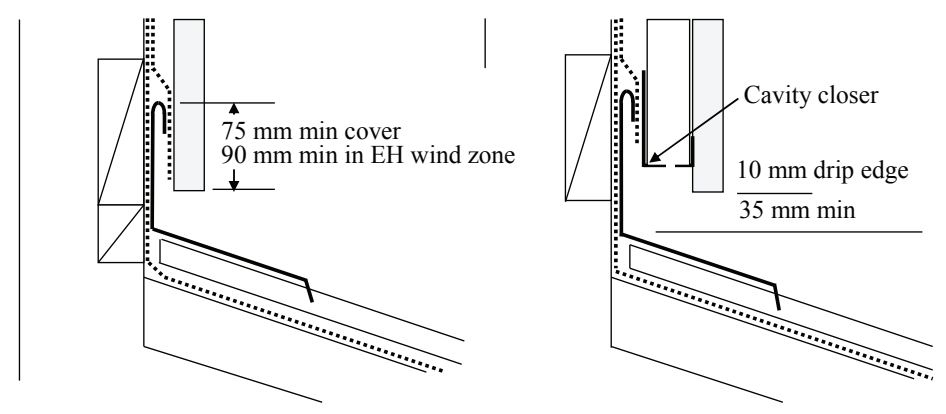

Figure 3. A selection of flashings used in NZ buildings and taken from E2/AS1.

\section{Leakage Characteristics of a Horizontal Cladding Jointer}

The leakage characteristics of a plastic $\mathrm{H}$ jointer were measured between two cladding panels fixed to cavity battens as illustrated in Figure 4. 


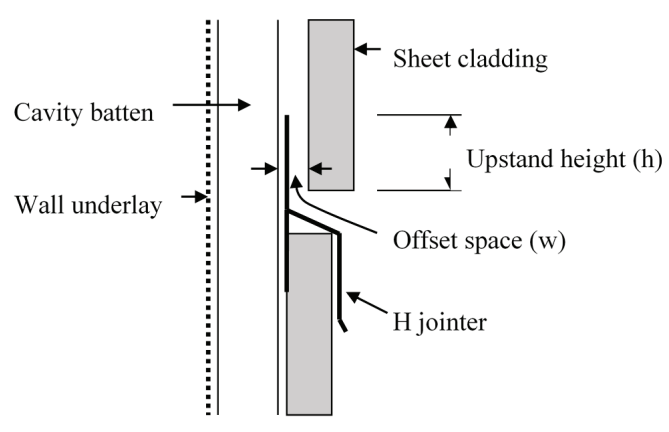

Figure 4. Sectional view through an $\mathrm{H}$ jointer between two sheets of wall cladding.

This is similar to the $\mathrm{Z}$ flashing illustrated in Figure 3 between plywood panels on a cavity wall except that it does not include a hem now required in E2/AS1 in the extra high wind zone. There are two main leakage paths in the $\mathrm{H}$ jointer as follows:

- Leakage between the up-turned leg of the jointer and the cladding. In particular the significance of the offset space width $(w)$, the height of the upstand and the presence or absence of a hem folded into the upstanding leg;

- Leakage over the top of the lower cladding past the lower legs of the $\mathrm{H}$ jointer-not investigated here.

\subsection{Significance of Offset (Air Gaps) between the Jointer and the Cladding}

The air pressure difference corresponding to the onset of leakage through the $\mathrm{H}$ jointer was measured for a range of upstand heights by cutting down the upper leg of the jointer to 15,35 and $60 \mathrm{~mm}$. A $530 \mathrm{~mm}$ wide section of jointer (spanning between cavity battens fixed to framing) was sprayed with water at $3.4 \mathrm{~L} / \mathrm{m}^{2}$ and the air pressure difference adjusted over the range 0-500 Pa until leakage was detected above the jointer. The pressure at which water reached the top of the upstand are shown in Figure 5 as 140, 294 and 500 Pa for the three upstand heights 15, 35 and $60 \mathrm{~mm}$.

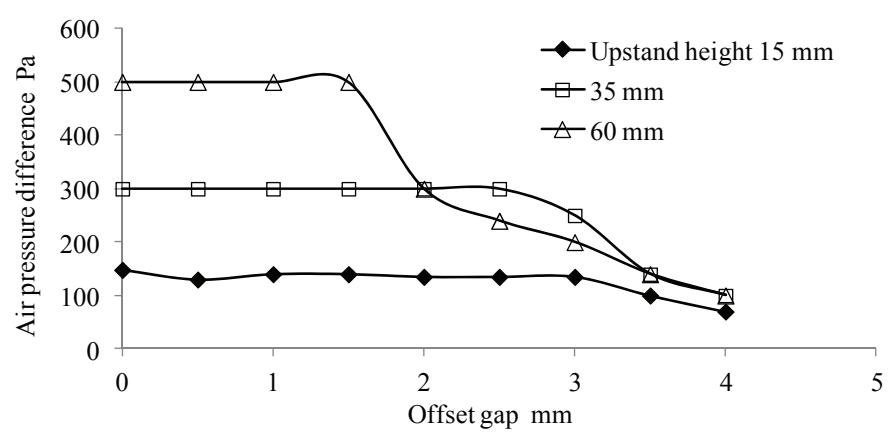

Figure 5. Air pressures at which water leakage occurrs through the $\mathrm{H}$ jointer as a function of upstand height and the offset gap width. 
The pressures needed to spill water over the upstand were a little higher than this $(147,300$ and $588 \mathrm{~Pa}$ ) because of the additional pressure needed to overcome surface tension. When the offset gap $w$ was increased beyond 2-3 mm, air carried water leakage was observed bubbling above the upstand. Figure 5 shows air carried water leaks occurring at lower pressures as the offset gap is increased for all three upstand heights.

\subsection{Significance of a Hem on the Upstand of the Jointer}

The PVC H jointer was replaced with folded aluminium flashings having upstand heights of 35 and $60 \mathrm{~mm}$ and with and without a hem formed as shown in Figure 3. In this case water leakage rates were measured gravimetrically over a period of one minute by weighing an absorbent strip placed just above the flashing. In all cases the cladding was held against the flashing upstand $(w=0)$ although the flashings with a hem will have formed a $3 \mathrm{~mm}$ gap between and most of the flashing upstand and the back of the cladding. With a $1.3 \mathrm{~g} / \mathrm{m} \cdot \mathrm{s}$ runoff rate over the joint, static pressure leakage characteristics for the 35 and $60 \mathrm{~mm}$ high flashings were as shown in Figure 6 to be similar to those measured earlier for the PVC H jointer with the onset of leakage at 300 and $500 \mathrm{~Pa}$. The onset of leakage was found to be independent of the hem, but once again, it was possible to reach onset leakage pressures as high as 370 and $580 \mathrm{~Pa}$ by carefully spring loading the hem of the 35 and $60 \mathrm{~mm}$ high flashings against the cladding. Although these measurements were conducted using standard construction materials, it is likely that the tolerances achieved in the laboratory were tighter than would be seen in buildings, and that onset leakage pressures in the 100-300 Pa range indicated in Figure 5 for offset gap widths above $2 \mathrm{~mm}$ are more likely where no special care is taken to hold the flashing against the cladding. Higher upstand dimensions do bring weathertight performance advantages but only where air carried water leakage can be eliminated with a tighter fit between flashing and cladding. Onset leakage pressures of 100-300 Pa are higher than the 50 Pa "wet wall" test pressure applied in E2/VM1 to the field of the cladding and on this basis there is little argument for increasing upstand heights.

Results of a more detailed study of the $35 \mathrm{~mm}$ high flashings applying static and fluctuating pressures are presented in Figure 7. Here, the water leakage rate is plotted against the peak air pressure difference and appears to indicate leakage rates at lower pressures as the frequency of the applied pressure increases. In fact, there was insufficient adjustment in the stroke of the fluctuating piston to keep the amplitude of the applied pressure independent of frequency and so the following additional analysis has been required. 


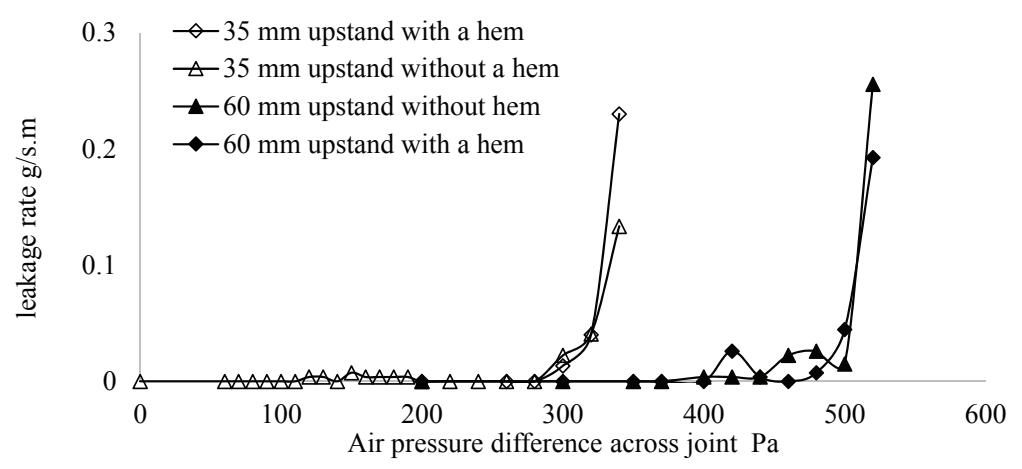

Figure 6. Steady pressure leakage characteristics of flashings with 35 and $60 \mathrm{~mm}$ upstands with and without hems.

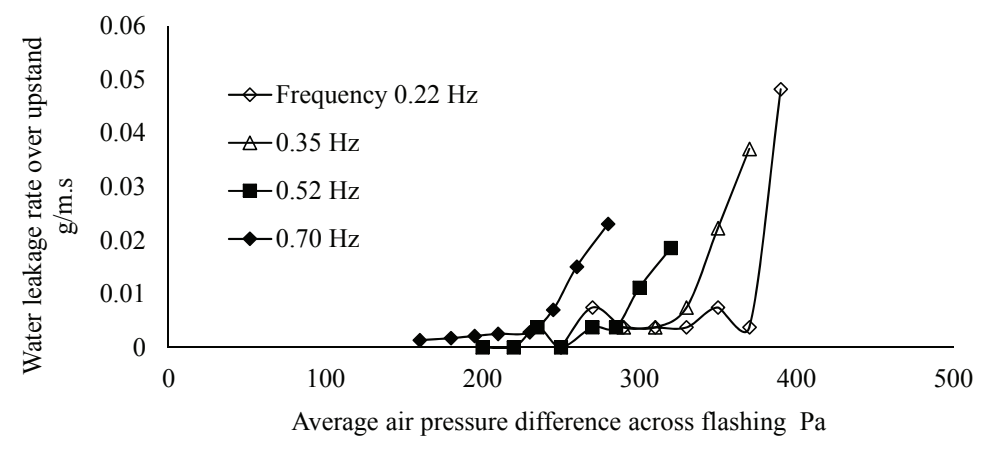

Figure 7. Dynamic leakage characteristics of flashings with a $35 \mathrm{~mm}$ upstand and a hem.

Observations showed that water accumulating inside and relaxing out of the joint was in phase with with pressure fluctuations up to $0.7 \mathrm{~Hz}$, suggesting that the leakage rate at any time in the cycle might simply be calculated from the steady pressure leakage rate function and the applied sinusiodal pressure as follows:

The applied pressure difference $\Delta p=A+B \sin \omega t$ Where $\Delta p=$ the applied pressure difference $(\mathrm{Pa}) ; A=$ the average pressure $(\mathrm{Pa}) ; B=$ the amplitude of pressure fluctuation $(\mathrm{Pa}) ; \omega=$ the angular velocity of the fluctuating pressure $(\mathrm{rad} / \mathrm{s}) ; L=$ the instantaneous joint leakage rate $(\mathrm{g} / \mathrm{m} \cdot \mathrm{s})$ and average leakage rate $\left(L_{\mathrm{av}} \mathrm{g} / \mathrm{m} \cdot \mathrm{s}\right)$, where the static leakage function is a function of the pressure $L=f(\Delta p)$ and the average leakage rate $L_{\mathrm{av}}=\frac{\omega}{2 \pi} \int_{0}^{2 \pi / \omega} f(A+B \sin \omega t) \mathrm{d} t$.

Calculated and measured leakage rates are plotted in Figure 8 for the $35 \mathrm{~mm}$ flashing with a hem. For these measurements, the hem was spring loaded against the back of the cladding to improve measurement repeatability, and average leakage rates were calculated numerically using the appropriate steady pressure leakage function.

The slope of the fitted line in Figure 8 is 1.06 with $r^{2}=0.75$, indicating that leakage rates calculated on the basis of there being no significant inertia in the physical system agree reasonably well with 
measured data up to a frequency of $0.7 \mathrm{~Hz}$. Higher frequencies than this tend to fall outside the power spectrum density of atmospheric turbulence (Kareem and Cermak [12]) and outside the range of frequencies considered relevant in pressure equalisation studies (Burnett and Straube [13]). Consequently the frequency of dynamic pressures applied in weathertightness tests is generally below $0.7 \mathrm{~Hz}$, e.g., 0.2 to $0.3 \mathrm{~Hz}$ in E2/AS1. A model that factors in the supply rate of water to the joint, the inertia of water filling the joint and the relaxation time for water draining from the joint has not been pursued.

A similar dynamic pressure response was seen with the same height flashing $(35 \mathrm{~mm})$ without a hem. In this case, leakage rates were higher because it was less easy to clamp the flashing against the cladding over its entire length. Once again, there was no evidence for frequency-dependent leakage characteristics below $0.7 \mathrm{~Hz}$.

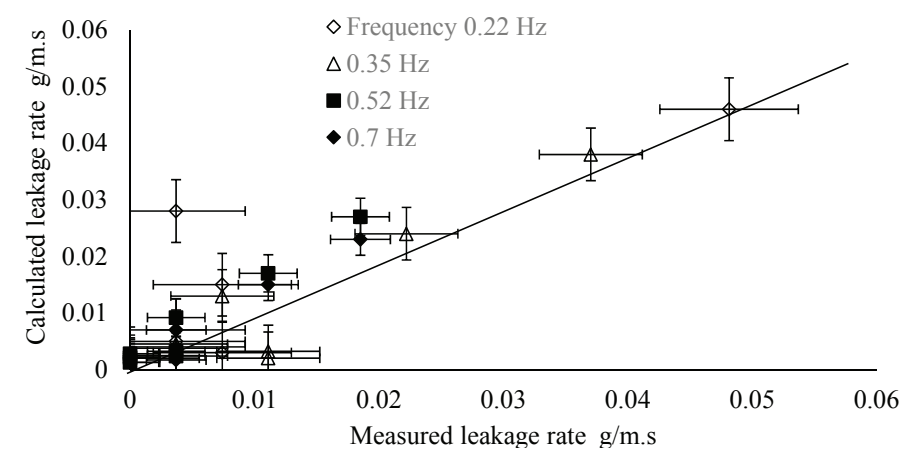

Figure 8. A comparison of measured and calculated leakage rates with fluctuating air pressures and showing experimental uncertainties.

\section{Leakage Characteristics of Cavity Closer in a Window Head Joint}

A window head joint was assembled as in Figure 9 but with the capacity to adjust the position of the upper cladding in relation to the window head flashing. This allowed for some variations in joint dimemsions, in particular, the gap between cladding and flashing $(g)$.

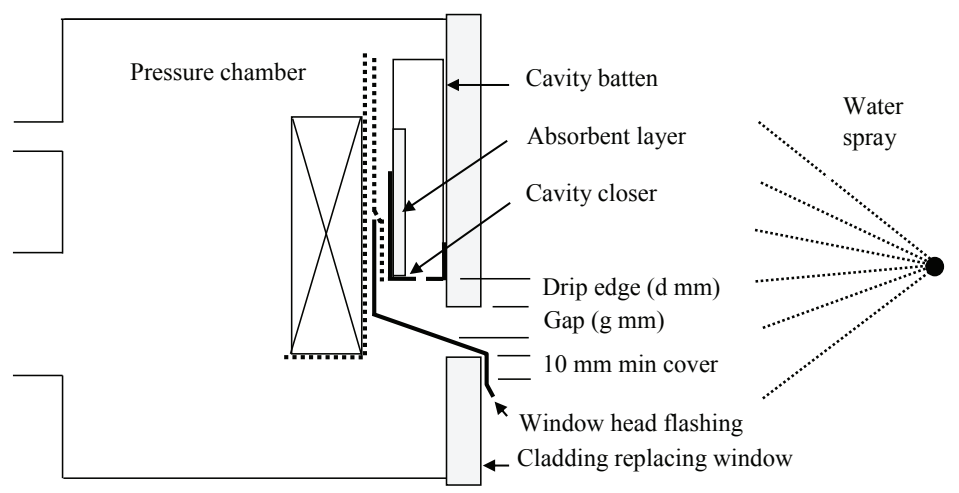

Figure 9. Experimental window head joint with cavity closer and variable joint dimensions. 
There are four potential water leakage paths above the window flashing in Figure 9 as follows:

- Between the head flashing upstand and the cavity closer which is similar to the leakage path examined earlier;

- Up through vent openings in the cavity closer as examined here;

- Past the stop ends on the head flashing — not dealt with here;

- Under the flashing over the lower cladding (or window head - not dealt with here).

The upstand height of the head flashing above the base of the cavity closer is shown in Figure 3 to be $20 \mathrm{~mm}$ (45 $\mathrm{mm}$ in an extra high wind zone) but in practice, the upstand on commercially available cavity closers is around $75 \mathrm{~mm}$, significantly increasing the effective upstand against water leaking through vent openings in the cavity closer. The $1500 \mathrm{~mm}^{2} / \mathrm{m}$ vent area at the base of the cavity closer was made up of a series of slots $3 \mathrm{~mm}$ wide by $13 \mathrm{~mm}$ long and exceeded the minimum vent area of $1000 \mathrm{~mm}^{2} / \mathrm{m}$ required by E2/AS1. Water leakage rates through the cavity closer were measured as a function of the steady air pressure across the joint and plotted against the distance travelled up the upstand. This was achieved by segmenting the absorbent layer into 9 parallel strips that could be individually weighed and assigned to a height above the cavity closer. The water spray rate was $3 \mathrm{~L} / \mathrm{min} \cdot \mathrm{m}^{2}$ as required by E2/VM1 and Figure 10 plots deposition rates against height above the cavity closer.

It is clear that pressures above the $50 \mathrm{~Pa}$ wet wall test pressure were needed to drive water through the cavity closer and onto the dry side of the cavity although the leakage onset pressures are considerably lower than for the $\mathrm{H}$ jointers discussed earlier. In fact the onset leakage pressures for leakage above $35 \mathrm{~mm}$ and $60 \mathrm{~mm}$ are in the range 100-125 Pa compared with 300 and $500 \mathrm{~Pa}$ for the $\mathrm{H}$ jointer, indicating that the airtightness of the joint and other aspects of joint configuration are important.

In practice, a significant proportion of wind pressure on a wall will lie across other components such as the internal lining and underlay, especially above a window which effectively partitions the wall cavity for pressure moderation across wet joints in the cladding. Secondly, it is important to acknowledge the value of ventiltion drying in both cavity walls and with direct fixed weatherboard claddings. While the vents associated with window head flashings might reduce onset pressures for water leakage, the pressures are still well above the "wet wall" test pressure of $50 \mathrm{~Pa}$ in E2/VM1. The potential for ventilation drying in cavity walls and behind direct fixed weatherboard walls is shown in WALLDRY-NZ (Bassett [11]) to provide the secondry water management needed to cope with even quite leaky claddings and to offset some loss in onset leakage pressures across window head flashings. For joints such as the window head flashing studied here, the water leakage measured and plotted in Figure 10 have yet to be compared with the capacity for ventilation drying and drainage from the joint.

It was observed that water leakage past the cavity closer depended on the runoff rate past the window head. High runoff rates tended to fill the joint, restricting the air flow into the cavity and allowing for wind pressures to carry water through vents in the cavity closer. This suggested that the water leakage rate may depend on dimensional factors that might be optimised to improve the water 
leakage characteristics of the head joint. The following factors that have been investigated in sequence:

- Dependency on runoff rate over the joint achieved with a range of water spray nozzles;

- The gap between cladding and flashing $(g)$;

- Whether the pressure was applied statically or dynamically.

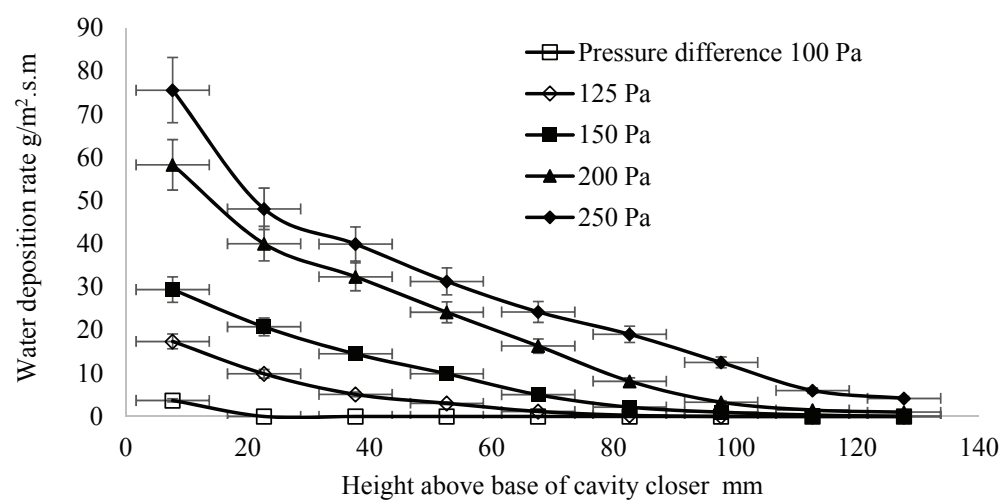

Figure 10. Deposition rate of water as a function of height above the cavity closer.

\subsection{Dependency of Onset Leakage Pressures on Runoff Rate}

The net water leakage rate into the cavity closer was measured at runoff rates $0.6-12 \mathrm{~g} / \mathrm{m} \cdot \mathrm{s}$ over the window head joint (equivalent to surface flow rates of 0.08 to $1.7 \mathrm{~L} / \mathrm{m}^{2} \cdot \min$ on the limited wall area above the head joint). Figure 11 plots the steady pressure difference at which water first penetrated the cavity closer against the runoff rate over the window head joint. During these measurements, the joint dimensions were fixed at those shown in Figure 9 with a $5 \mathrm{~mm}$ gap between cladding and the head flashing. Two water leakage regimes were observed. Above a runoff rate of $2 \mathrm{~g} / \mathrm{m} \cdot \mathrm{s}$, water tended to bridge across the joint, partially filling the space below the cavity closer and allowing water to be carried past the cavity closer at relatively low pressure differences. At runoff rates below $2 \mathrm{~g} / \mathrm{m} \cdot \mathrm{s}$ the joint drained out, leaving an unobstructed air path to the cavity closer. With the vent unobstructed there was some water leakage above the cavity closer at higher pressures due to air carried spray, but the leakage rates were much smaller than those that were measured when the runoff rate exceeded $2 \mathrm{~g} / \mathrm{m} \cdot \mathrm{s}$. 


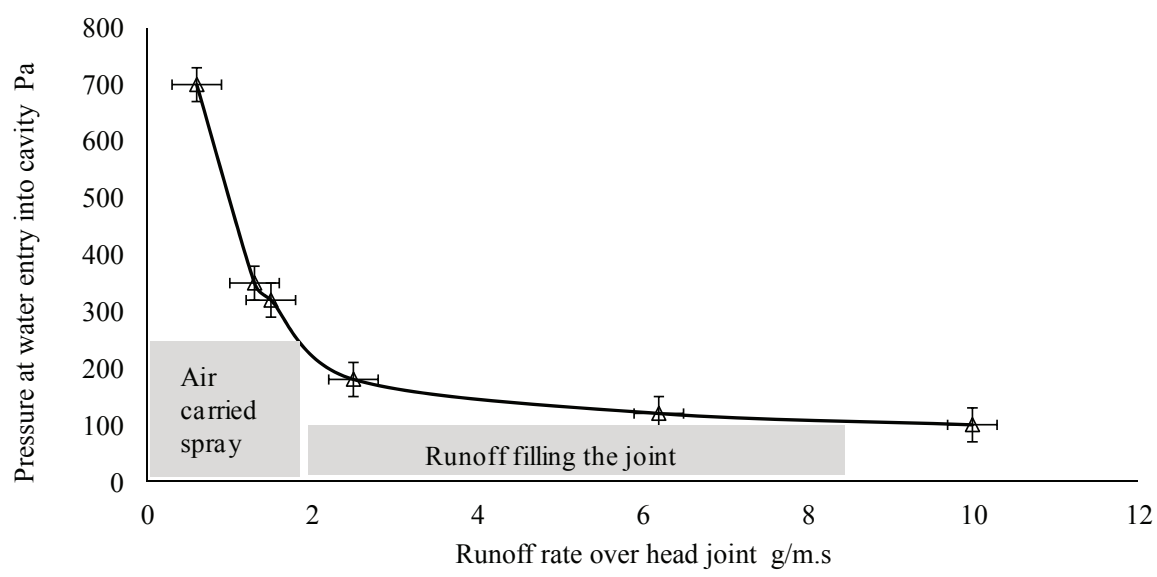

Figure 11. Pressure difference at the onset of leakage through a window head joint as a function of runoff rate over the joint.

\subsection{Dependency of Onset Leakage Pressures on Gap between Cladding and Head Flashing}

The dependency on runoff rate in Figure 11 suggests that the cladding to head flashing dimension might be an important factor in the weathertight performance of this joint and a separate sequence of leakage measurements were carried out to investigate this possibility with the gap width $g$ ranging from 1 to $9.6 \mathrm{~mm}$. Figure 12 shows how the onset leakage pressure increased with larger gap $(g)$ dimensions leading to the joint draining out more effectively. This could be worth exploring more in the context of taller buildings exposed to higher wind pressures and surface runoff rates. Below a gap width of $5 \mathrm{~mm}$ the leakage performance of the joint appears to improve marginally, but it has to be remembered, this is at the expense of free drainage from the joint.

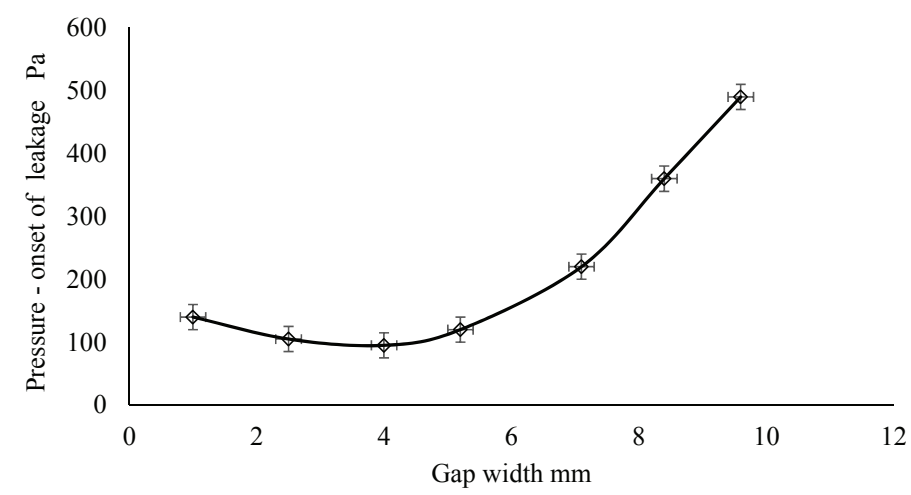

Figure 12. Dependence of onset leakage pressure on gap width for the window head joint and the runoff rate set at $2 \mathrm{~g} / \mathrm{s} \cdot \mathrm{m}$.

Table 1 gives a pictorial view of leakage through a window head joint with varying surface run-off rates and gap dimensions between upper cladding and head flashing. It shows that increasing 
the cladding to head flashing gap $(g)$ improved the capacity of the joint to deal with high run-off rates, but this might also be achieved in other ways, for example, by adding a small kick-out to the lower edge of the cladding to deflect water from the opening.

Table 1. A pictorial view of leakage through a window head joint with varying surface run-off rates and gap dimension $(g)$ between upper cladding and head flashing.

Water Leakage Description
Case A: Gap width $(\mathrm{g})=5 \mathrm{~mm}$ and the run-off rate is less than $2 \mathrm{~g} / \mathrm{m} \cdot \mathrm{s}$.
The gap is not significantly occluded by run-off, and the flashing drains out effectively.
Above a wind pressure of $200-300 \mathrm{~Pa}$, air flow through the joint entrains small droplets,
which pass through into the cavity.

\section{Leakage with Dynamic Applied Wind Pressures}

Water leakage of the window head joint was measured with dynamically applied air pressures in the $0-0.6 \mathrm{~Hz}$ range. During these measurements, the geometry of the joint was as shown in Figure 9 and the run-off rate over the joint was constant at $2.5 \mathrm{~g} / \mathrm{m} \cdot \mathrm{s}$. The pressure amplitude was adjusted to fall to a zero minimum where possible, but at low frequencies, there was not enough travel in the oscillating piston to achieve the full pressure amplitude. The water leakage rates into the cavity closer have been plotted in Figure 13 against peak air pressure for five frequencies in the range $0-0.6 \mathrm{~Hz}$.

There is a clear trend for the leakage characteristic to converge on the static pressure result at low frequencies, but it diverges towards much lower leakage rates at higher frequencies. This is a different result to that for $\mathrm{H}$ jointers between direct-fixed wall panels. Here, the leakage rate appeared to be in phase with varying wind pressures and was insensitive to frequency sensitivity in the range $0-0.7 \mathrm{~Hz}$. 


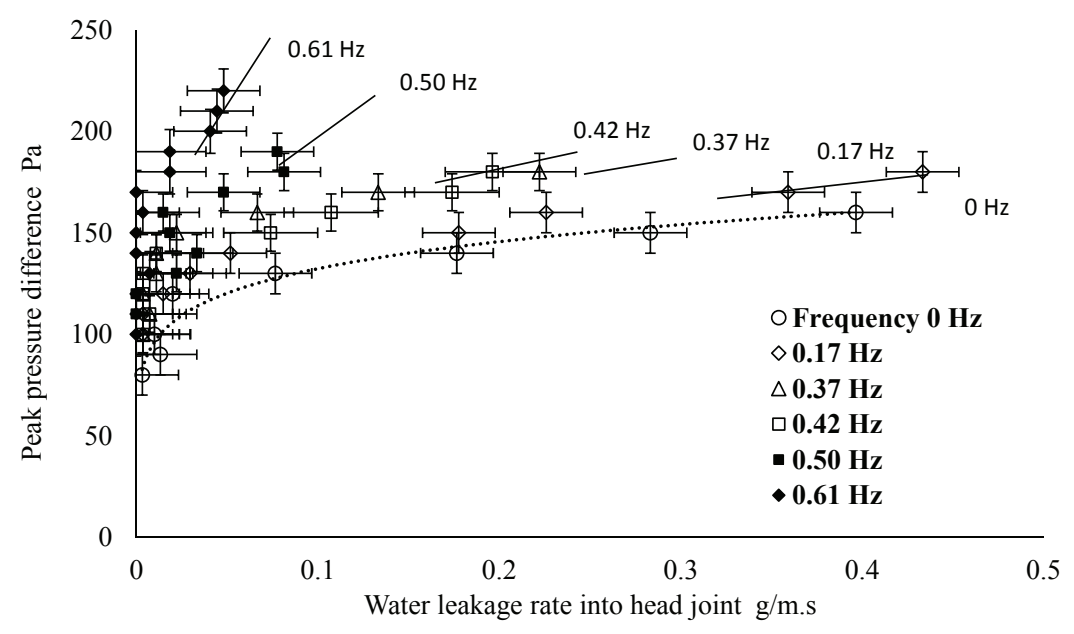

Figure 13. Frequency dependent water leakage into a window head joint.

A two-phase flow analysis of the water leakage process in construction joints is outside the scope of this study, but the question of whether the leakage rates shown in Figure 13 can be derived from the instantaneous leakage rates as for $\mathrm{H}$ and $\mathrm{Z}$ jointers has been addressed in Figure 14. Here, the instantaneous leakage rates over a full cycle have been calculated from the steady pressure results as described earlier and the calculated values compared with measured leakage rates. It is clear that leakage rates for frequencies below $0.4 \mathrm{~Hz}$ can be calculated from the steady pressure leakage data, but at higher frequencies, inertia and the time constant of the joint cavity filling and drainage processes will have to be accounted for. There is room for further development of the leakage rate dependency on fluctuating pressures.

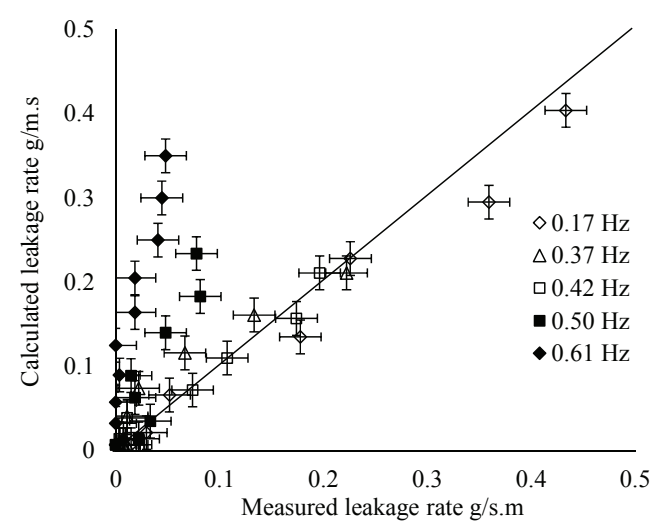

Figure 14. A comparison of measured and calculated leakage rates using a simplified model. 


\section{Leakage Characteristics of an Apron Flashing to Roof Joint}

An apron flashing was assembled as shown in Figure 15 with the cavity wall details given in Figure 3. The apron flashing was simplified to a single piece in folded aluminium with an upstand height (distance from base of cladding to top of hemed upstand) of $75 \mathrm{~mm}$.

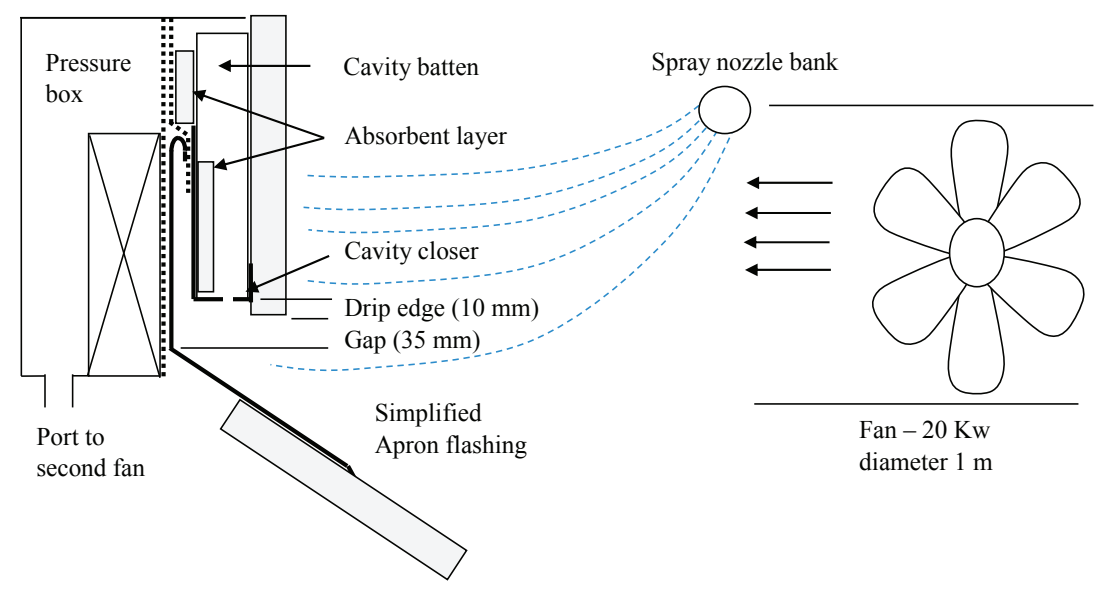

Figure 15. Experimental apron flashing joint between roof.

The complete wall specimen measured $2.4 \mathrm{~m}$ by $2.4 \mathrm{~m}$ and a portion of the wall cladding was made removable to retreve and weigh the absorbent layer. The leakage characteristics of the joint were measured statically as a function of runoff rate following the methods used for the window head flashing. Then the static spray bar was replaced with a $1 \mathrm{~m}$ diameter fan capable of driving water spray at the joint with air velocities up to $17 \mathrm{~m} / \mathrm{s}$. This allowed the leakage due to wind carried rain to be compared from that due to runoff.

Four potentially significant water leakage paths past the apron flashing in Figure 16 are as follows:

- Between the flashing upstand and the cavity closer;

- Up through vent openings in the cavity closer;

- Past the ends of the apron flashing - not dealt with here;

- Under the flashing over the roof deck—not dealt with here.

Leakage characteristics of a $1.2 \mathrm{~m}$ section of joint were measured with a $75 \mathrm{~mm}$ flashing upstand, a cavity closer upstand of $75 \mathrm{~mm}$ against the flashing and a smaller $18 \mathrm{~mm}$ upstand against the back of the cladding. The vent area in the cavity closer was $1500 \mathrm{~mm}^{2} / \mathrm{m}$ made up of a series of slots $3 \mathrm{~mm}$ wide by $13 \mathrm{~mm}$ long. It was the same cavity closer present in the window head joint studied earlier. Runoff rates over the joint were in the range 3-60 g/m·s.

Water leakage rates were measured inside the cavity closer against the taller leg at the back of the cavity, and directly above the flashing. No water entry was detected above the $75 \mathrm{~mm}$ flashing upstand, due to the hem fitting tightly against the cavity closer and effectively closing off air leakage paths in this area. As with the window head flashing, water entered through ventilation holes in the 
cavity closer and leakage rates were measured at four runoff rates and air pressure differences in the range $0-1300 \mathrm{~Pa}$ and plotted in Figure 16. The joint behaved like a window head joint with a large gap between cladding and flashing that prevented the joint from filling up with water at even the highest runoff. The measured leakage rates were small compared to those measured into the window cavity closer and were entirely due to small droplets of spray carried by air flows through ventilation holes in the cavity closer.

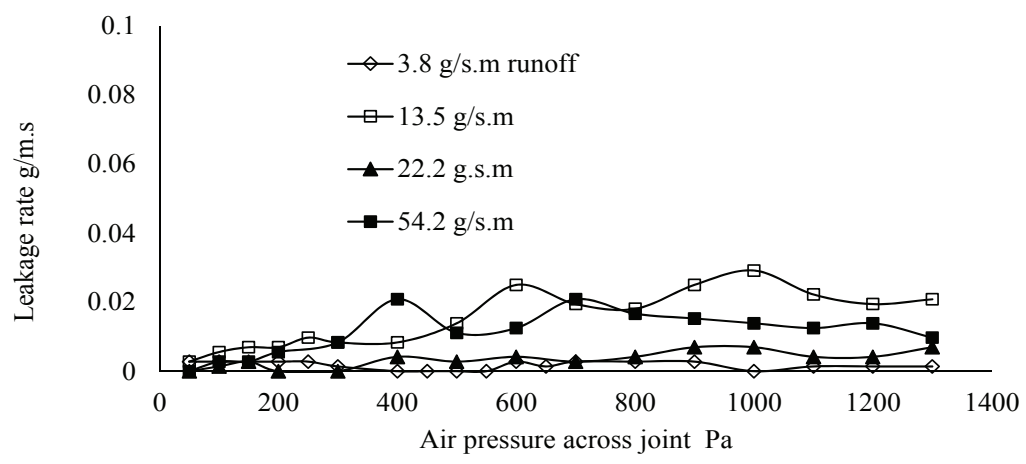

Figure 16. Water leakage rates through the cavity closer above a saddle flashing.

\section{Wind Driven Water Leakage past the Apron Flashing}

The specimen was positioned in front of the large fan and the static air pressure measured at the joint. At maximum fan speed this was $168 \mathrm{~Pa}$ (equivalent to an air speed of $16.7 \mathrm{~m} / \mathrm{s}$ and well short of the pressures applied in E2/VM1 for buildings located in very high and extra high wind zones). The E2/VM1 test sequence does not simulate wind carried driving rain and so the measurements described here are at best exploratory. The sprays positioned above the fan outlet delivered $16 \mathrm{~L} / \mathrm{min}$ or an average of $2.8 \mathrm{~L} / \mathrm{m}^{2} \cdot \min$ over the specimen.

The rate of water entry into the cavity closer was measured as a function of the air pressure difference across the joint and the height of water penetration on the plane of the wall underlay. As in the earlier measurements, there was no leakage past the top of the $75 \mathrm{~mm}$ apron flashing but significant quantities of water entered through vents in the cavity closer. The air pressure difference across the cavity closer was adjusted from the baseline $168 \mathrm{~Pa}$ in steps of $200 \mathrm{~Pa}$ to $1168 \mathrm{~Pa}$ using the second fan shown in Figure 15. Figure 17 shows the water entry rate plotted against height above the base of the cavity closer. This time, the water entry rate is plotted on a log scale to illustrate the large difference between water entering the joint and carried through the cavity closer to higher levels by air flows.

There were two processes at work carrying water into this joint. The first, involved rain drops bouncing off the apron flashing and carried by momentum into the base of the cavity closer. From here they travelled no further unless an air pressure difference greater than $100 \mathrm{~Pa}$ was present to drive water further into the joint. The second process involved smaller droplets entrained in air flows which travelled higher into the joint. The first process delivered large quantities of water and the second delivered much smaller water flows that were similar to those measured earlier with static 
sprays. It is clear that wind driven rain is a significant issue with apron flashings that will require further investigation in the context of tall buildings.

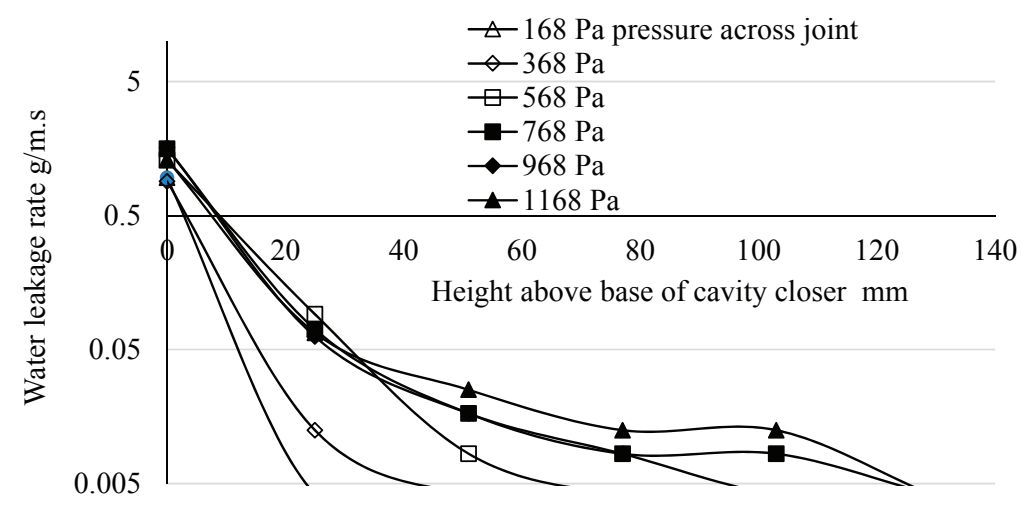

Figure 17. Mass flow of water reaching various heights above the base of the cavity closer of an apron flashing.

\section{Conclusions}

The water leakage characteristics of three flashed joints from the New Zealand Building Code approved document E2/AS1 have been measured. At this stage, the leakage characteristics are in a raw form and have yet to be interpreted in terms of weather data for mid-rise buildings in New Zealand. Water leakage rates were measured as a function of rain load, air pressure difference (statically and dynamically applied) and, in one case, with the rain load driven by high wind speeds. In all of the joints studied, the water leakage performance limit was found to depend on the presence of air leakage paths, either in the form of vents in cavity closers or due to some misfit between flashings and claddings. For this reason, it is likely that field performance will be well short of the leakage onset pressures measured here for ideal joints. Bearing in mind this limitation, the following conclusions were drawn:

- H jointers between sheets of cladding. With the flashing close fitting, the joint water penetration up to pressures close to the head of water equivalent of the up-stand height. In more realistic building applications where the gap between flashing and cladding might exceed 2-3 mm, air carried water leaks were seen at lower leakage onset pressures (100-300 Pa) with little dependency on up-stand height and the presence of a hem. Water leakage rates through tight-fitting joints were measured with dynamic pressure in the range of $0-0.7 \mathrm{~Hz}$. There was no detectable effect of inertia, with measured water leakage rates agreeing with calculations using static pressure leakage characteristics.

- Window head flashing in a cavity wall. The onset of water leakage through this joint occurred at $100 \mathrm{~Pa}$ as a result of air carried leakage through vents in the cavity closer. Leakage past the flashing upstand was much less significant when tightly fitted against the cavity closer. Both onset leakage pressures were much higher than the $50 \mathrm{~Pa}$ "Wet Wall" test pressure adopted in E2/VM1. The leakage onset pressure was also found to depend on the 
runoff rate over the joint and on the gap between the cladding and head flashing. In fact this gap might need to be increased to cope with runoff rates on tall facades. The dynamic leakage characteristics were quite different to those at static pressures, unlike the case for $\mathrm{H}$ jointers. Leakage rates at frequencies above $0.4 \mathrm{~Hz}$ were over predicted by the simple instantaneous pressure model and will therefore require a model involving the inertia of water in the joint and the filling and drainage time constants.

- Apron flashing between wall and roof. The $35 \mathrm{~mm}$ gap between the base of the wall cladding and the apron flashing prevented high runoff rates from accumulating water in the space below the cavity closer. As a consequence, only very small air carried water leaks through the cavity closer were detected up to very high air static pressure differences. Leakage rates a hundred times greater were measured with rain driven at the joint by $17 \mathrm{~m} / \mathrm{s}$ wind speeds. The new water entry process involved large droplets bouncing off the apron and entering the base of the cavity closer where water could be carried deeper into the joint by air flows. No leakage was detected between the apron flashing upstand $(75 \mathrm{~mm}$ above the base of the cladding) while it fitted tightly against the cavity closer upstand.

This study identified opportunities to improve the leakage characteristics of joints to handle runoff and higher wind pressures for buildings outside the scope of E2/AS1. This is, however, only a start for more comprehensive studies that will be needed to include fluctuating wind pressures and wind driven rain into the water leakage functions for joints. These studies will also compare measured leakage rates with the capacity for drainage and ventilation drying.

\section{Acknowledgments}

This work was funded by the Building Research Levy. The assistance of Mark Hearfield, and Roger Stanford is gratefully acknowledged.

\section{Author Contributions}

Both authors contributed to the experimental work and the first author wrote the paper.

\section{Conflicts of Interest}

The authors declare no conflict of interest.

\section{References}

1. Bassett, M.; Clark, S.; Camilleri, M. Building Weathertightness Failures-Associated Risk Factors. In Proceedings of the BETEC Symposium on Building Science Applications at Syracuse University, New York, NY, USA, 5 September 2006.

2. Hershfield, M. Survey of Building Envelope Failures in the Coastal Climate of British Columbia; Report for Canada Mortgage and Housing Corporation; Morrison Hershfield Ltd.: Ottawa, Canada, 1996. 
3. Approved Document E2/AS1 External Moisture; Department of Building and Housing: Wellington, New Zealand, 2005.

4. Hazleden, D.G.; Morris, P.I. Designing for Durable Wood Construction: The 4D's. In Durability of Building Components 8; NRC Research Press: Ottawa, Canada, 1999.

5. Approved Document E2/VM1 External Moisture; Department of Building and Housing: Wellington, New Zealand, 2005.

6. Ishikawa, H. An Experiment on the Mechanism of Rain Penetration through Horizontal Joints in Walls. In Proceedings of the 2nd International CIB/Rilem Symposium on Moisture Problems in Buildings, Paper 2.3.1., Rotterdam, The Netherlands, 10-12 September 1974.

7. Herbert, M.R.M. Open-Jointed Rain Screen Claddings; Building Research Establishment Current Paper 89/74; Building Research Establishment (BRE): Watford, UK, 1974.

8. Herbert, M.R.M.; Harrison, H.W. New Ways with Weatherproof Joints; Building Research Establishment Current Paper 90/74; Building Research Establishment (BRE): Watford, UK, 1974.

9. Lacasse, M.A.; O’Connor, T.J.; Nunes, S.; Beaulieu, P. Report from Task 6 of MEWS Project Experimental Assessment of Water Penetration and Entry into Wood-Framed Wall Specimens; Research Report IRC-RR-133; Institute for Research in Construction, National Research Council of Canada: Ottawa, Canada, 2003.

10. Sahal, N.; Lacasse, M.A. Proposed method for calculating water penetration test parameters of wall assemblies as applied to Istanbul, Turkey. Build. Environ. 2008, 43, 1250-1260.

11. Bassett, M.; McNeil, S.; Overton, G. WALLDRY-NZ: An Educational Tool for Rain Loads and Ventilation Drying in Cavity Walls. In Proceedings of the 5th International Building Physics Conference, Kyoto, Japan, 28-31 May 2012.

12. Kareem, A.; Cermak, J.E. Wind Tunnel Simulation of Wind-Structure Interactions. In International Instrumentation Symposium, 24th, Albuquerque, N. Mex., May 1-5, 1978, Proceedings. Part 2; Instrument Society of America: Pittsburgh, PA, USA, 1978.

13. Burnett, E.; Straube, J. Vents, Ventilation Drying and Pressure Moderation; Canada Mortgage and Housing Corporation Research Report; Canada Mortgage and Housing Corporation: Ottawa, Canada, 1995. 


\title{
Comparison of NZ's Energy Efficiency Regulation and Verification Assumptions to Real Building Loads and Operation
}

\section{Shaan Cory, Michael Donn and Andrew Pollard}

\begin{abstract}
The New Zealand building design industry assumes various building model inputs for the consumption of energy through lighting and appliances. It also makes assumptions regarding when these energy consumers are considered to be "turned on". This paper aims to better inform industry energy modellers about the real load and operation of real commercial buildings in New Zealand when compared to New Zealand Standard energy efficiency requirements and assumptions. The paper presents a set of New Zealand relevant commercial building operation information. Typical operation information is provided for three commercial building types: (1) Office; (2) Retail; and (3) Mixed/Other. The information provides low, typical, and high installed building load and operation pattern scenarios for the three building types. The typical data presented in this paper is significantly different to the load requirement and operation modelling assumptions presented in the New Zealand Building code. The results established in this paper are informed by data gathered in the Building Research Association of New Zealand (BRANZ) Building Energy End-Use Study (BEES). The purpose of BEES is to increase knowledge on energy use patterns for the entire New Zealand building stock. The intention of this paper is to disseminate the established knowledge that will eventually update the assumptions used in New Zealand commercial energy models.
\end{abstract}

Reprinted from Buildings. Cite as: Cory, S.; Donn, M.; Pollard, A. Comparison of NZ's Energy Efficiency Regulation and Verification Assumptions to Real Building Loads and Operation. Buildings 2015, 5, 116-129.

\section{Introduction}

This paper presents typical, high and low building load densities and patterns of use for commercial buildings in New Zealand. Patterns of use refers to the daily use profiles of the load presented as the percentage of load "on" at different time intervals throughout the day. The goal is to better inform building energy modellers about typical lighting and equipment end uses. In the process, this work highlights the differences between the assumptions made when using the modelling verification method to meet the New Zealand Building Code (NZBC) and what is occurring in real buildings [1].

The research is a part of the Building Research Association of New Zealand (BRANZ) Building Energy End-Use Study (BEES). The purpose of BEES is to monitor and analyse the energy and water consumed by non-residential buildings around New Zealand. The project ran for 6 years [2]. BEES aims to provide more insightful knowledge of energy use patterns for the entire New Zealand commercial building stock [3]. Real data is collected within selected premises through the BEES programme from the monitoring of temperature, humidity, light levels, $\mathrm{CO}_{2}$ levels, occupant and equipment schedules, internal loads and fuel consumption. 
This paper is one of two reports which documents better informed building energy models for the New Zealand design industry. The building load and patterns of use conclusions determined in this study informed the creation of prototypical commercial building energy models. The prototypical models are documented on the BRANZ BEES website. The models provide a base case for the building design industry to assess the impact of building load and operation on their buildings energy performance.

\section{Lack of Measured Building Operation Information}

Currently, when analysing building energy performance, the New Zealand building design industry must make assumptions about various building model inputs based on New Zealand Standard (NZS) verification method for displaying compliance with NZBC. Inputs to their performance calculation models rely on assumptions about the energy consumed by lights and appliances. Energy modellers assessing energy may use the NZS 4243 Lighting Power Density (LPD) value as a base scenario for what is currently installed in commercial buildings. NZS values are used because there is no other reliable information on the typical building load power densities found in real commercial buildings.

Half of the commercial buildings in New Zealand were built before 2000. This pre-dates the NZBC and NZS 4243 clause regulating the LPD. This can cause modellers to enter into their performance calculation of an existing building retrofit analysis assumed values that may not be typical. Additionally, there is no such NZBC regulated density value for other building loads, such as office equipment and hot water. This results in modellers inputting a value for an Equipment Power Density (EPD) and Hot Water Power Density (HWPD) that is a best guess or an assumption based on what is included in the NZS 4243 modelling method to prove a building design meets the NZBC. Table 1 displays the power density "assumptions" found in the NZS 4243.

Table 1. New Zealand Standard (NZS) 4243 Power densities. Table created using [4,5].

\begin{tabular}{|c|c|c|c|}
\hline \multirow[b]{2}{*}{ Building Type } & \multicolumn{3}{|c|}{ Power Densities $\left(\mathrm{W} / \mathrm{m}^{2}\right)$} \\
\hline & $\begin{array}{l}\text { Regulated Lighting } \\
\text { Power Density (LPD) }\end{array}$ & $\begin{array}{l}\text { Assumed Equipment } \\
\text { Power Density (EPD) }\end{array}$ & $\begin{array}{c}\text { Assumed Hot Water } \\
\text { Power Density (HWPD) }\end{array}$ \\
\hline Office & $12 \mathrm{~W} / \mathrm{m}^{2}$ & $8.1 \mathrm{~W} / \mathrm{m}^{2}$ & Not required \\
\hline Retail & $\begin{array}{c}8-16 \mathrm{~W} / \mathrm{m}^{2} \text { depending on } \\
\text { retail type }\end{array}$ & $\begin{array}{c}1.1-2.7 \mathrm{~W} / \mathrm{m}^{2} \text { depending on } \\
\text { retail type }\end{array}$ & Not required \\
\hline Mixed/Other & $\begin{array}{c}8-18 \mathrm{~W} / \mathrm{m}^{2} \text { depending on } \\
\text { use type }\end{array}$ & $\begin{array}{c}1.1-8.1 \mathrm{~W} / \mathrm{m}^{2} \text { depending on } \\
\text { use type }\end{array}$ & Not required \\
\hline
\end{tabular}

Note: The Retail and Mixed/other power densities have a range due to the different building types.

The same problem occurs for the operation patterns of building loads. No measured information for the patterns of use of building loads in real buildings is currently published. As a result, an energy modeller is left with little option but to assume when equipment is turned "on" and when it is turned "off" based on the modelling assumptions set in the NZS 4243 energy modelling method. Table 2 presents the operation pattern assumptions found in the NZS 4243 energy modelling 
method for different building types. There is no evidence that these assumptions will be relevant to how real commercial buildings in New Zealand consume energy.

Table 2. NZS 4243 Lighting and Plug Load patterns of use. Table adapted from [4].

\begin{tabular}{ccccccc}
\hline \multirow{2}{*}{$\begin{array}{c}\text { Building } \\
\text { Type }\end{array}$} & \multirow{2}{*}{ Day Type } & \multicolumn{5}{c}{ Patterns of Use (\% of Load "on") } \\
\cline { 3 - 7 } & & $\mathbf{1 2}$ p.m.-8 a.m. 8 a.m.-11 a.m. & 11 a.m.-6 p.m. & $\mathbf{6}$ p.m.-10 p.m. & $\mathbf{1 0}$ p.m.-12 p.m. \\
\hline \multirow{3}{*}{ Office } & Week & $5 \%$ & $90 \%$ & $90 \%$ & $30 \%$ & $5 \%$ \\
& Saturday & $5 \%$ & $30 \%$ & $15 \%$ & $5 \%$ & $5 \%$ \\
& Sunday & $5 \%$ & $5 \%$ & $5 \%$ & $5 \%$ & $5 \%$ \\
\hline \multirow{2}{*}{ Retail } & Week & $5 \%(15 \%)$ & $90 \%(40 \%)$ & $90 \%(90 \%)$ & $50 \%(90 \%)$ & $5 \%(50 \%)$ \\
(Restaurant) & Saturday & $5 \%(15 \%)$ & $90 \%(30 \%)$ & $90 \%(80 \%)$ & $30 \%(90 \%)$ & $5 \%(50 \%)$ \\
& Sunday & $5 \%(15 \%)$ & $40 \%(30 \%)$ & $40 \%(70 \%)$ & $5 \% 60 \%)$ & $5 \%(50 \%)$ \\
\hline \multirow{2}{*}{ Mixed/Other } & Week & $5 \%$ & $90 \%$ & $90 \%$ & $5 \%$ & $5 \%$ \\
& Saturday & $5 \%$ & $24 \%$ & $5 \%$ & $5 \%$ & $5 \%$ \\
& Sunday & $5 \%$ & $5 \%$ & $5 \%$ & $5 \%$ & $5 \%$ \\
\hline
\end{tabular}

Notes: Retail has two patterns of use presented as restaurants a different to general retail. Also,

Mixed/Other are the assumptions for a warehouse, but it could be a mixture of all three use types.

New Zealand is not alone with regard to these assumptions and lack of information. The current state of the art in prototypical building models can be found in a US set of prototypical building models [6]. These are based on informed engineering judgements about "typical" or "design" values for building loads and their operation. As part of the BEES modelling research, a set of EnergyPlus Template models [7] were developed which followed the same format as the U.S. prototypical models. The aim of the template models was to reduce the difficulties associated with energy modelling, particularly relating to the ease and speed of creating a model to produce reliable results [8]. The BEES models have the same short comings in that they are built on assumptions about the building loads and their operation. If the BEES models were updated using the measured data presented in this report, they would be more advanced than any prototypical models currently found. The biggest single advantage of updating the models with measured building load and operation patterns is that they offer the potential for building design teams to examine the risk that a predicted building performance is dependent on the assumptions made about people, lighting and equipment used in the design modelling. There have been criticisms of high performance designs due to the fact that they only perform well in the particularly narrow focused situation "assumed" during the performance modelling [9]. The low and high building loads data from BEES allows designers to quickly test realistic design scenarios and establish how robust their design concept is.

\section{Methodology}

This study uses the measured data for lighting, equipment and hot water energy use from the BEES survey of commercial premises. The BEES team collected data over a four year period from 2008 to 2012. Energy was measured at one minute intervals for a 2-3 weeks' period in a representative sample of New Zealand commercial building premises. The weekly periods were spread throughout the year for different buildings. This means that the energy results could be from 
a two week period in summer or a two week period in the winter. Because this report only deals with lighting, equipment and hot water the outdoor conditions will have a minimal impact on energy use. This was the case for lighting also as there were no daylight induced electric lighting dimming in any of the premises. For site measurement technique and protocol used for monitoring refer to BEES Years 1 and 2 report [3].

This report presents the power density and patterns of use for lighting, equipment, and hot water. The lighting used is strictly indoor lighting to provide task lighting to the occupants. Equipment is made up of a number of appliances that are used by occupants to undertake day to day tasks and enable businesses to provide a service. Appliances include computers, printers, servers, refrigerators, chillers, water coolers, water boilers, phone systems, security systems, ovens, stove tops, deep fryers, and other appliances used in the day to day use of commercial buildings. Hot water energy use covers the energy used to provide hot water for domestic use, such as hand washing and showers, and commercial use, such as dishwashing.

All results were calculated using the measured average weekday and weekend 10 min interval load. Each 10 min interval measured was averaged against other weekday and weekend 10 min intervals. The power densities were calculated by dividing the maximum measured load (for lighting, equipment, and hot water), by the monitored floor area. The operation patterns were calculated by dividing the measured load by the maximum measured load to establish the percentage of load "on" during that 10 min interval.

A typical, a low and a high energy load scenario is presented in this report. The typical scenario is the Median (50th percentile) load and pattern of use found across the sample of building premises. Because the sample has outliers which can differ greatly from other values, the median provides a good indicator of the most typical value in the sample [10]. The Low scenario is the 10th percentile of measured load and pattern of use across the sample of premises. The High scenario is the 90th percentile of measured load and pattern of use across the sample of premises. The sample sizes vary between the lighting, equipment and hot water assessments and are displayed in Table 3.

The time intervals used in the patterns of use analysis were decided by assessing natural breaks across the whole data set in the amount of load "on" during each hour. For example, 12 a.m. to 5 a.m. is used as there was less than 10 percent difference in load across these hours. The hours were then averaged to get a percentage value of load "on" across these $5 \mathrm{~h}$. These data are presented for 3 different building types. The load was split by building use type because NZS 4243 regulates load for different building types, and the use of different commercial buildings results in very different building load attributes. Table 4 displays the three building types assessed (Office, Retail, and Mixed/Other) and the detailed uses found within each of the three building types.

A further breakdown was made when assessing the lighting, equipment and hot water power densities. The breakdown assessed the impact of building floor area size. Building floor area size was assessed because NZS 4243 regulates building loads for only "large buildings" which it deems are $300 \mathrm{~m}^{2}$ or greater. The assessment of size indicates whether building size has a significant impact on the building loads. Table 5 displays the breakdown of the different building floor area sizes assessed 
(small, medium and large), and the percentage of the total commercial building floor area each building size makes up.

Table 3. Sample sizes used to calculate typical, low and high values.

\begin{tabular}{ccccc}
\hline End-Use & All Buildings & Office & Retail & Mixed/Other \\
\hline Lighting & 101 & 35 & 29 & 37 \\
Equipment & 83 & 28 & 22 & 33 \\
Hot Water & 30 & 9 & 7 & 14 \\
\hline
\end{tabular}

Table 4. Building type categories.

\begin{tabular}{llll}
\hline Building Type & Office & Retail & Mixed/Other \\
\hline \multirow{2}{*}{$\begin{array}{l}\text { Specific building use } \\
\text { types found in each } \\
\text { building type }\end{array}$} & Office-type use & $\begin{array}{l}\text { Retailing use, Motor vehicle sales } \\
\text { and services, Liquor outlets including } \\
\text { taverns, Service stations, Tourist-type }\end{array}$ & $\begin{array}{l}\text { A mixture of office and } \\
\text { retail use types. As well } \\
\text { attractions }\end{array}$ \\
\hline
\end{tabular}

Table 5. Building size groupings.

\begin{tabular}{cccc}
\hline Building Size & Small & Medium & Large \\
\hline Building floor area range & 5 to $649 \mathrm{~m}^{2}$ & 650 to $3499 \mathrm{~m}^{2}$ & Over $3500 \mathrm{~m}^{2}$ \\
Percentage of all commercial floor area & $20 \%$ & $40 \%$ & $40 \%$ \\
\hline
\end{tabular}

\section{Results and Discussion}

All results displayed in the graphs below are documented on the BEES website [7]. The results presented on the website enable energy modellers to enter the values into energy models more precisely.

\subsection{Lighting Power Density (LPD)}

Figure 1 displays the typical, high and low LPDs' for an average commercial building (white), Offices (light grey), Retail (dark grey), and Mixed/Other commercial use types (black). As can be seen, Retail use types have the highest installed LPD's. This is followed by Offices, and Mixed/Other commercial use types. This trend mimics the trend of the maximum allowable LPD set by the NZS 4243 verification method [5]. It is worth noting that the typical LPDs found in Offices are slightly lower $\left(11 \mathrm{~W} / \mathrm{m}^{2}\right)$ than the value set by NZS 4243 of $12 \mathrm{~W} / \mathrm{m}^{2}$ [5]. By comparison, the LPDs found in Retail premises are similar to the minimum set out in NZS 4243 . For Retail NZS 4243 requirement is $8-16 \mathrm{~W} / \mathrm{m}^{2}$ depending on retail use type, and the typical measured LPD across all retail use types is $14 \mathrm{~W} / \mathrm{m}^{2}$ [5]. The typical LPD measured in Mixed/Other commercial use type premises is much lower than the NZS 4243 requirement. NZS 4243 allows for a maximum of $8-18 \mathrm{~W} / \mathrm{m}^{2}$ depending on use type, and the typical measured LPD was $6 \mathrm{~W} / \mathrm{m}^{2}$. Mixed/Other commercial LPDs are below the sample average. Also, the high scenario $\left(20 \mathrm{~W} / \mathrm{m}^{2}\right)$ for Mixed/Other commercial LPD's is not significantly different to the Retail $\left(26 \mathrm{~W} / \mathrm{m}^{2}\right)$ and Office $\left(21 \mathrm{~W} / \mathrm{m}^{2}\right)$ high values as the low and typical scenarios. 


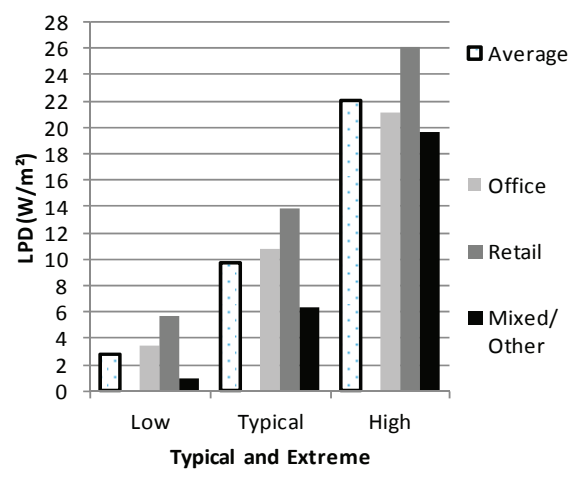

Figure 1. Typical, High and Low LPDs' for different commercial building types.

Figure 2 presents the typical, high and low LPDs' for Small buildings (light grey), Medium sized buildings (dark grey), and Large buildings (black) to assess if size has an impact on the installed LPD. As can be seen, Medium sized buildings have the highest measured LPDs with a typical value of approximately $13 \mathrm{~W} / \mathrm{m}^{2}$. The LPDs found in Small and Large buildings were the same with $8 \mathrm{~W} / \mathrm{m}^{2}$. The high scenario results suggest that lighting was most dense in Medium and Small sized buildings due to the large building high LPD value being half the Small and Medium sized buildings LPDs. This was likely attributed to smaller buildings being more associated with Retail uses which have higher lighting demands.

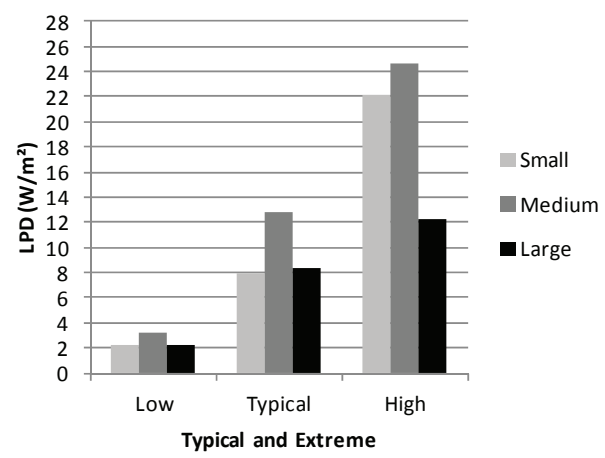

Figure 2. Typical, High and Low LPDs' for different sized commercial buildings.

\subsection{Lighting Operation Patterns}

Figure 3 displays the Typical (green), High (orange), Low (blue) and NZS 4243 (black) patterns of lighting use for Offices, Retail and Mixed/Other commercial use types. 


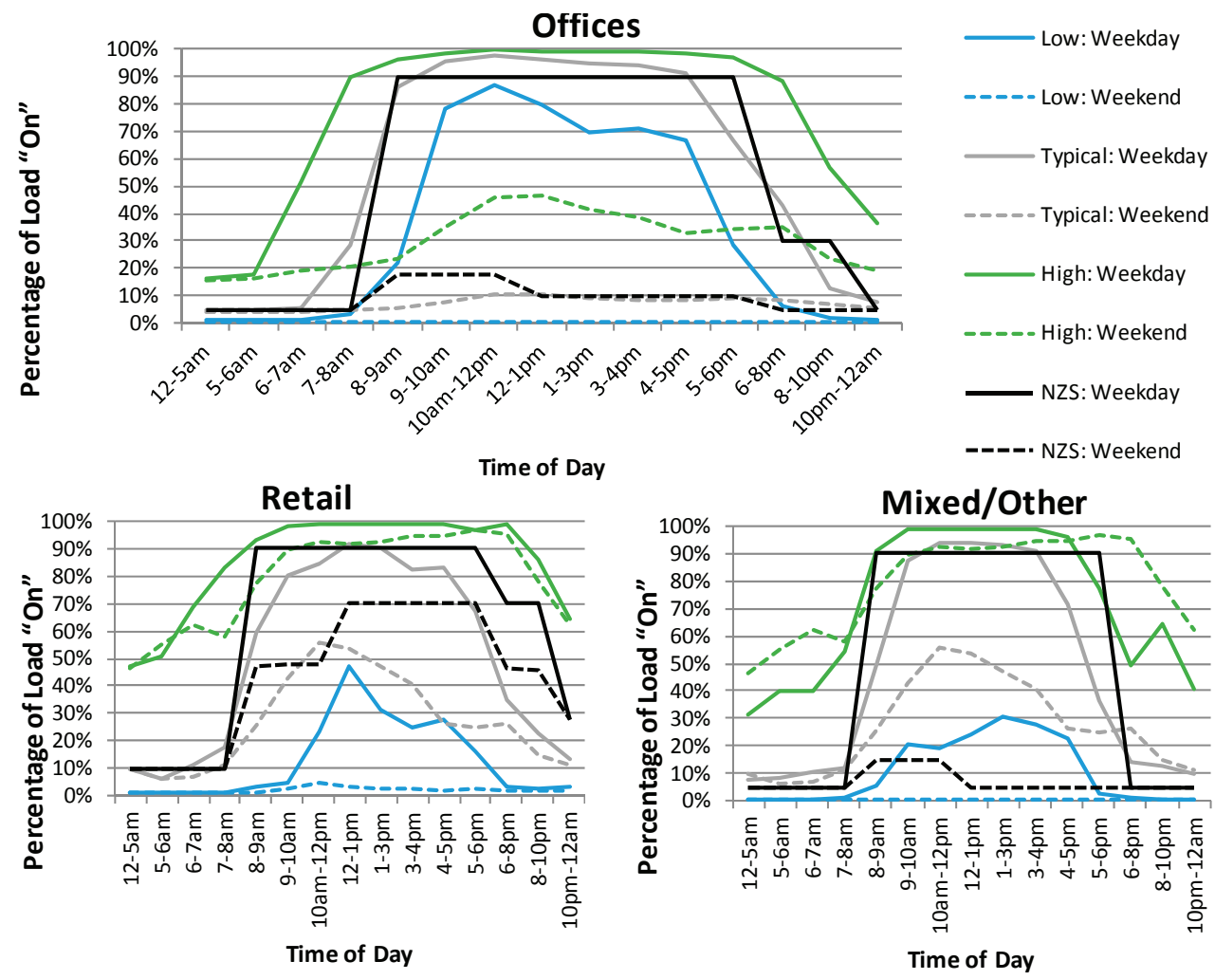

Figure 3. Typical and Extreme Lighting Patterns' of use for different commercial building types. Notes: The Retail pattern of use was an average between the NZS 4243 retail and restaurant patterns. The weekend patterns were an average of the NZS 4243 Saturday and Sunday patterns of use.

As can be seen, Offices have the largest difference in usage patterns between weekdays and weekends. The typical lighting usage of an Office was higher ( $80 \%-90 \%$ turned "on") than Retail and Mixed/Other commercial uses during the weekdays (70\%-80\% turned "on"). Retail and Mixed/Other commercial uses have less difference in usage patterns between weekdays and weekends (20\%-40\% difference) when compared to Offices (60\%-70\% difference). This fits with expectations that Retail and Mixed/Other commercial use buildings have more intense and longer weekend hours compared to Office buildings. Interestingly, the high use Retail and Mixed/Other use types have almost as intense weekends as weekdays compared to the lighting in high use Offices.

The typical lighting use pattern for Offices was similar to the NZS 4243 assumed pattern of use. However, the Retail and Mixed/Other patterns sit between the typical and high scenario patterns of use. If the NZS 4243 assumptions were used in an energy model, then the model would slightly overestimate the amount of lighting turned "on" in a typical building. 


\subsection{Equipment Power Density (EPD)}

Figure 4 displays the Typical, High and Low EPDs' for the average commercial building (white), Offices (light grey), Retail (dark grey), and Mixed/Other commercial use types (black). Mixed/Other commercial use types have the lowest typical EPD with $5 \mathrm{~W} / \mathrm{m}^{2}$. Offices were the second lowest equipment focused use type with a typical EPD of $8 \mathrm{~W} / \mathrm{m}^{2}$. Retail has the highest typical EPD of $15 \mathrm{~W} / \mathrm{m}^{2}$. This suggests that Retail buildings are the highest equipment energy intensive building type. This finding makes sense as Retail buildings contain premises which are often used for food sales that include refrigeration and cooking. Retail also has a significantly larger high scenario EPD than the other building types, with an EPD of $58 \mathrm{~W} / \mathrm{m}^{2}$. The typical EPD of Offices was similar to the NZS 4243 assumption of $8 \mathrm{~W} / \mathrm{m}^{2}$. However, the other two building types typical EPD's were well above the NZS 4243 assumed EPDs (refer to Table 1 for NZS 4243 assumptions). This highlights the difference between the theoretical EPD and the real buildings EPD. The impact this would have on energy models would be large considering the amount of internal heat gains that would not be modeled using the NZS 4243 assumptions.

Figure 5 presents the typical, high and low EPDs' for Small buildings (light grey), Medium sized buildings (dark grey), and Large buildings (black) to assess the impact of building size on the installed EPD. Small sized buildings were the most equipment focused with a typical EPD of $13 \mathrm{~W} / \mathrm{m}^{2}$. Medium and Large buildings have a substantially lower density of equipment installed with a typical EPD of 6 and $7 \mathrm{~W} / \mathrm{m}^{2}$ respectively. This trend follows in the high EPD scenario. However, small buildings have a much larger EPD $\left(59 \mathrm{~W} / \mathrm{m}^{2}\right)$ when compared to both Medium and Large sized buildings, which have high EPDs of 38, and $18 \mathrm{~W} / \mathrm{m}^{2}$ respectively. This suggests that Small and Medium sized buildings are dominated by Retail loads while larger buildings are dominated by Office loads.

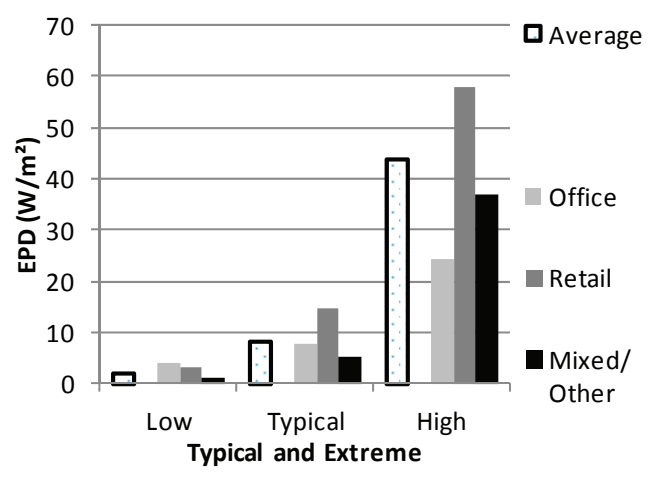

Figure 4. Typical, High and Low EPDs’ for different commercial building types. 


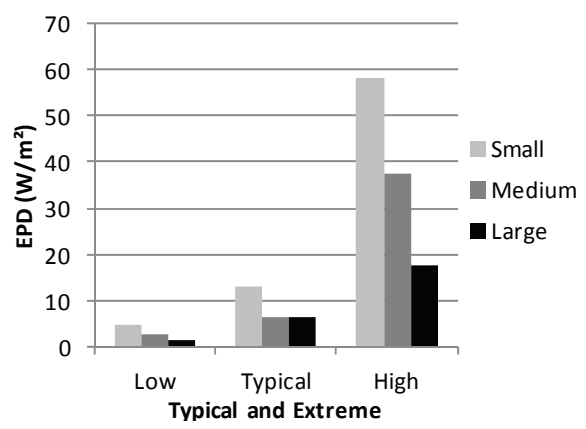

Figure 5. Typical, High and Low EPDs' for different sized commercial buildings.

\subsection{Equipment Operation Patterns}

Figure 6 displays the Typical (green), High (orange), Low (blue) and NZS 4243 (black) patterns of equipment use for Offices, Retail and Mixed/Other commercial use types.

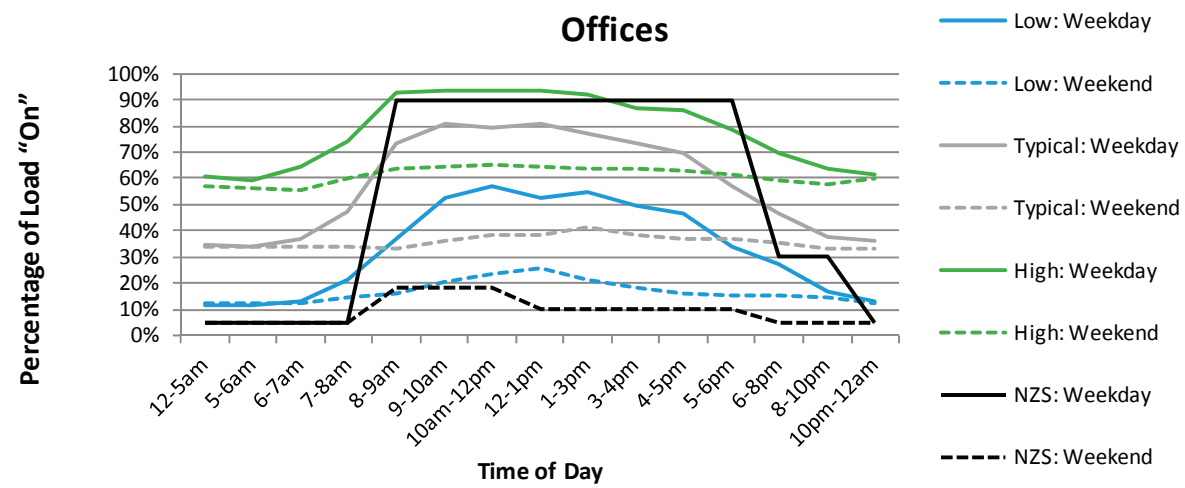

Retail

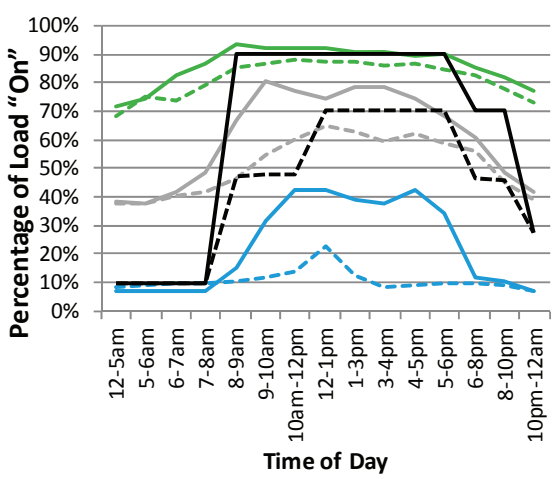

Other

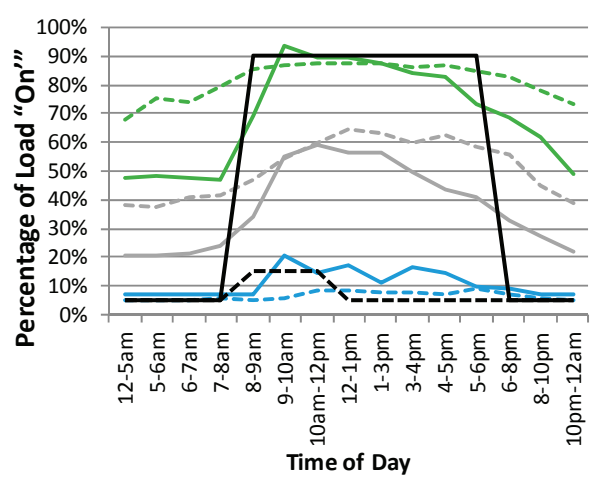

Figure 6. Typical and extreme equipment patterns' of use for different commercial building types. Note: The Retail pattern of use was an average between the NZS 4243 retail and restaurant patterns. The weekend patterns were an average of the NZS 4243 Saturday and Sunday patterns of use. 
Offices have the least energy intensive patterns of use as seen by the amount of load that was left "on" during unoccupied periods. This was shown by the weekend and night load percentages. Also, Offices have the biggest difference between night and daytime load patterns. This was highlighted by the larger peaks in equipment energy use of approximately $30 \%-50 \%$ during daytime hours. Office weekday patterns of use have a consistent peak of use whereas their weekend schedules were more or less consistently flat throughout the weekend. This was shown by the weekend day loads not being significantly different to the overnight use. Retail buildings have the most energy intensive patterns of use. This was highlighted by the larger percentage of equipment "on" and for longer periods of time. This was further reinforced by the fact that Retail buildings have higher EPD's (refer to Figure 4). Furthermore, the greater the energy intensity of a Retail premise, the closer the weekend pattern of use was to the weekday pattern. The daytime pattern of use peak in Retail premises ( $20 \%-30 \%$ more equipment "on") was not as large when compared to Offices $(30 \%-50 \%$ more equipment "on"). This could be due to the large refrigeration loads running consistently throughout the whole day, while only a small number of appliances were turned "on" during occupied hours. Unlike Office and Retail, the weekend patterns of use were higher than the weekday patterns of use in Mixed/Other commercial use buildings. The weekday and weekend on Mixed/Other use buildings also have a definite daytime peak of equipment use. Consistent with Retail, the daytime peaks were not as large as for Offices; however, there was a bigger daytime peak during weekdays when compared to the weekends. Also, the weekend loads were more consistently "on" and the weekend night loads were greater than the weekday night loads. This could be due to premises with restaurant and other food type having longer weekend hours.

The results indicate that the common perception among energy auditors, but not in building codes or standards, of equipment being left "on" during unoccupied hours is true. Half of the installed equipment load in Offices was left on overnight. Over a yearly period this equates to a large sum of energy that is essentially wasted. Coupled with this being typical across the commercial building stock, it would seem there is large potential for energy savings if equipment is turned off over night.

The NZS 4243 patterns of use assume that 10\%-20\% more equipment is turned "on" during daytime hours in Office and Retail buildings, and 10\%-30\% more in Mixed/Other buildings. The NZS 4243 patterns of use sit between the Typical and High scenarios for all three building types during daytime hours and around the Low scenario during night hours. Therefore, if the NZS 4243 assumptions are used in the model, they would overestimate the amount of equipment turned "on" during daytime and underestimate equipment turned "on" during night hours in a typical building.

\subsection{Hot Water Power Density (HWPD)}

Figure 7 displays the typical, high and low HWPDs' for average commercial buildings (white), Offices (light grey), Retail (dark grey), and Mixed/Other commercial use types (black). Retail buildings have the largest HWPD with $7 \mathrm{~W} / \mathrm{m}^{2}$ for a typical building and $24 \mathrm{~W} / \mathrm{m}^{2}$ for high use buildings. This was most likely due to the food use types associated with Retail using larger amounts of hot water compared to Offices and Mixed/Other commercial use types. Office and 
Mixed/Other use type HWPD's were below the average for commercial buildings with 3 and $2 \mathrm{~W} / \mathrm{m}^{2}$ respectively. The high scenario HWPDs for Office and Mixed/Other building types were significantly lower than the Retail HWPD.

Figure 8 presents the typical, high and low HWPDs' for Small buildings (light grey), Medium sized buildings (dark grey), and Large buildings (black) to assess if size has an impact on the installed HW-PD. The typical HWPD was relatively similar for each of the three building size groups. However, the "high" scenario indicates that Medium-to-Large sized buildings have higher demand for hot water when compared to Small buildings. This may occur from larger buildings needing to service a greater number of occupants when compared to smaller buildings.

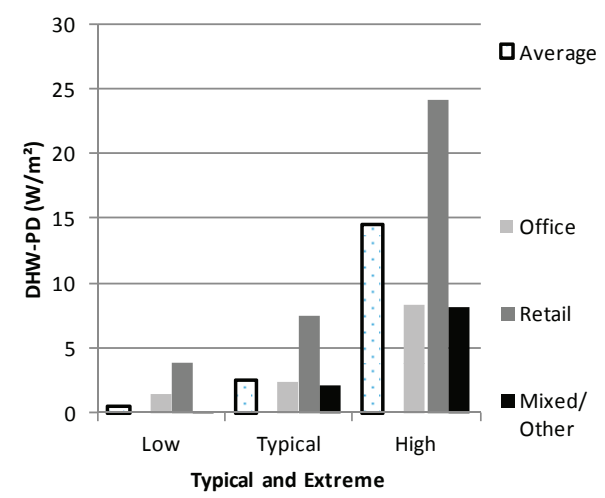

Figure 7. Typical, High and Low HWPDs' for different commercial building types.

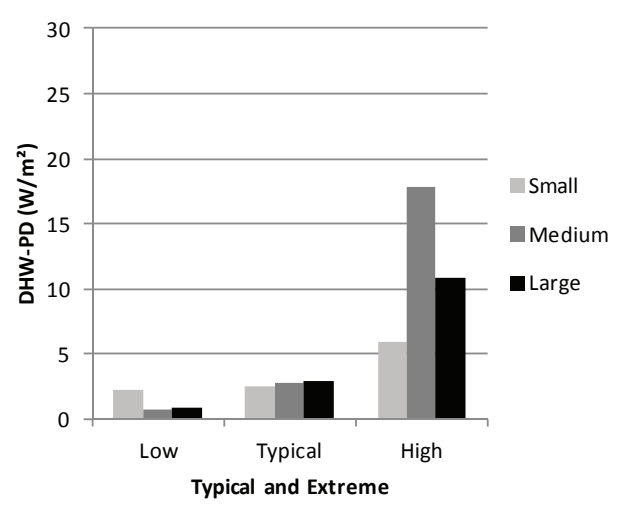

Figure 8. Typical, High and Low HWPDs' for different sized commercial buildings.

\subsection{Hot Water Operation Patterns}

Figure 9 displays the typical (green), high (orange) and low (blue) patterns of hot water use for Offices, Retail and Mixed/Other commercial use types. Office hot water energy use was very different between weekday and weekend, but also from nighttime to daytime use. Office hot water use was much lower overnight, by approximately $30 \%-40 \%$, as well as during the weekend with a 
$20 \%-40 \%$ smaller daytime peak of hot water energy usage. Retail hot water energy use patterns have consistent trends across both weekdays and weekends. The intensity of use between weekdays and weekends also does not change considerably (less than 10\%-20\%). Mixed/Other commercial use types consume more hot water energy in the weekends than during weekdays. This was consistent with the equipment patterns of use for Mixed/Other use building types.
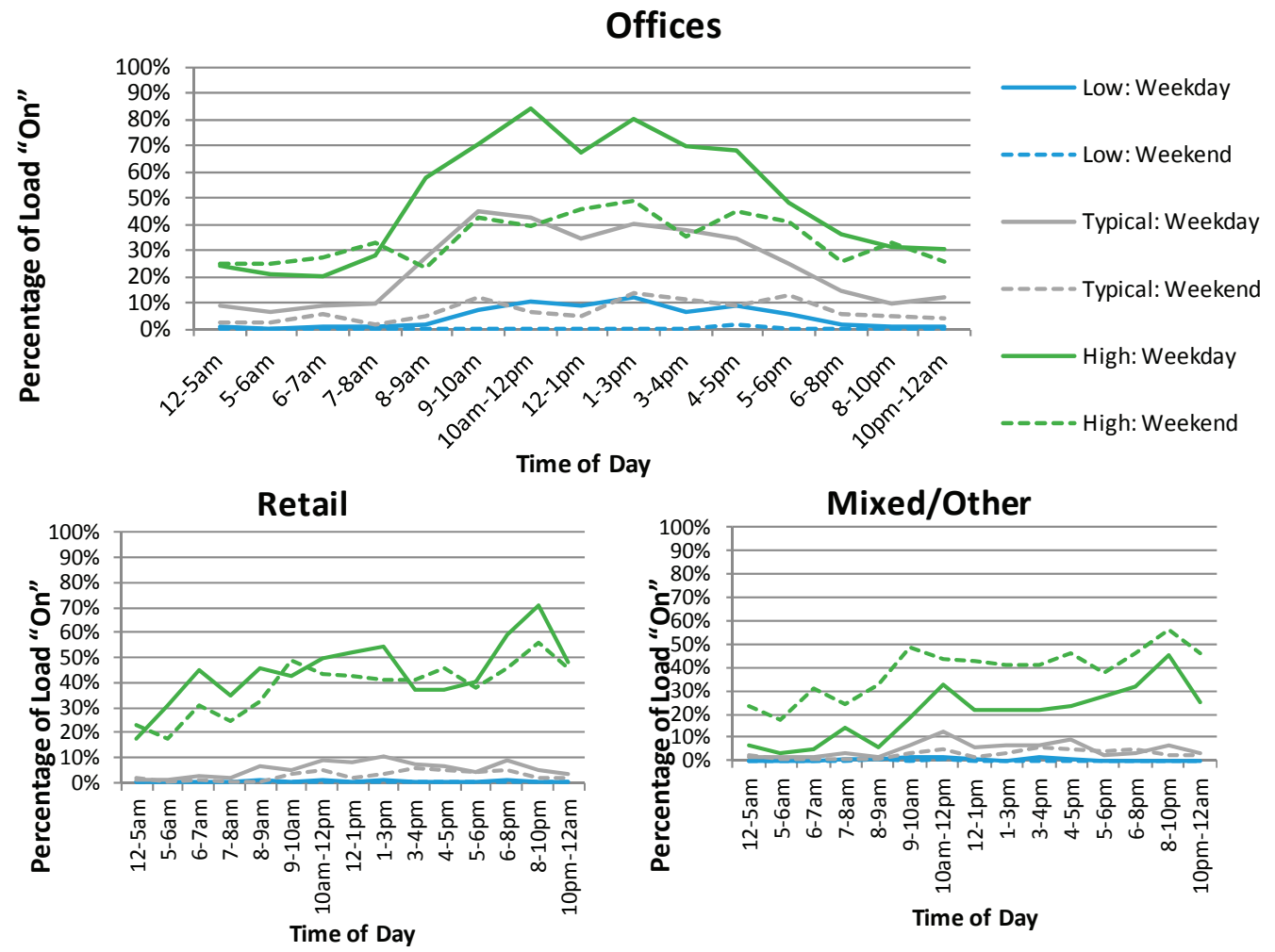

Figure 9. Typical and extreme DHW patterns' of use for different commercial building types.

\section{Conclusions}

This paper presents the typical, high and low building load densities and their operation patterns for commercial buildings in New Zealand. It fills the gap for the shortfall in information available for commercial building designers and environmental engineers. The shortfall relates to the lack of information regarding building loads found in real commercial buildings. Additionally, this paper compares the building loads found in real commercial buildings to the loads regulated in the NZS 4243 and the assumptions used in the modelling method for comparing energy use to prove the building code has been met.

The results established in this paper indicate that the current NZS 4243 values for power densities and associated schedules are not representative of existing building design in New Zealand. It has been found that the NZS 4243 values for the LPD are indicative of the typical LPDs 
found in existing buildings. It suggests that typical commercial buildings are designed to just meet the code and not to be any more energy efficient than they need to be. The Retail and Mixed/Other lighting patterns of use overestimate the amount of weekday lighting turned "on". The EPDs found in real buildings were significantly higher for Retail and Mixed/Other commercial use type buildings than the assumptions made in the NZS 4243 modelling method. This results in an underestimation of equipment energy use and internal heat gains if the NZS 4243 values were used. If concerned about assumptions affecting the performance of the building design, an energy modeller could double the power densities or reduce them by a third. By doing so, it roughly estimates the extreme lighting and equipment scenarios which occur across the commercial building stock.

Additionally, the common perception that equipment is left "on" during unoccupied hours is true. Half of the installed equipment load in Offices was left "on" overnight. Over a yearly period this equates to a large sum of energy that is essentially wasted. This combined with the typical values across the commercial building stock indicates that there is large potential for energy savings if equipment is turned off overnight in Offices.

The conclusions are relevant internationally. Building codes and standards suffer the same limitations as the New Zealand building standards which incorrectly assume no equipment or lighting use overnight. The U.S. prototypical models have same assumption limitations and it is likely these assumptions are used elsewhere internationally. These operational patterns were input as "good engineering judgement". It shows that good judgement needs to be informed and updated using the results presented in this thesis in the absence of a countries own experimental data (which is advised to be gathered). Furthermore, the High and Low scenario can be used as a way of altering prototypical models internationally. They can be used as a ratio to be used as a basis for overseas design as well.

These conclusions provided the basis for the creation of prototypical models, as well as the update of existing template models. The models are founded on the real building performance established in this study and the BEES studies. Each model contains New Zealand relevant materials, constructions, building loads, patterns of use, and HVAC system type and properties.

\section{Acknowledgments}

The authors would like to acknowledge the BEES team members and BEES project funders who enabled the analysis of the load and patterns of use data.

\section{Author Contributions}

Shaan Cory and Michael Donn conceived and designed the experiment; Shaan Cory performed the experiment; Shaan Cory analysed the data; Andrew Pollard contributed materials; Shaan Cory and Michael Donn wrote the paper.

\section{Conflicts of Interest}

The authors declare no conflict of interest. 


\section{References}

1. Department of Building and Housing. Compliance Document for New Zealand Building Code Clause H1: Energy Efficiency, 3rd ed.; Department of Building and Housing: Wellington, New Zealand, 2011.

2. BRANZ Ltd. Building Energy End-use Study (BEES), 31 March 2013. Available Online: http://www.branz.co.nz/BEES (accessed on 26 August 2013).

3. Isaacs, N.; Saville-Smith, K.; Bishop, R.; Camilleri, M.; Jowett, J.; Hills, A.; Moore, D.; Babylon, M.; Donn, M.; Heinrich, M.; et al. Building Energy End-Use Study (BEES) Years 1 \& 2; BRANZ Study Report SR 224 (2009); BRANZ: Judgeford, New Zealand, 2009.

4. Standards New Zealand. NZS 4243: Part 1: Energy Efficiency-Large Buildings: Building Thermal Envelope; Standards New Zealand: Wellington, New Zealand, 2007.

5. Standards New Zealand. NZS 4243: Part 2: Energy Efficeincy-Large Buildings: Lighting; Standards New Zealand, Wellington, New Zealand, 2007.

6. Torcellini, P.; Deru, M.; Griffith, B.; Benne, K.; Halverson, M.; Winiarski, D.; Crawley, D. DOE Commercial Building Benchmark Models; NREL/CP-550-43291; National Renewable Energy Laboratory (NREL): Golden, CO, USA, 2008.

7. BRANZ Ltd. BEES for Energy Modelling, 1 April 2013. Available Online: http://www.branz.co. nz/cms_display.php?sn=169\&st=1\&pg=9690 (accessed on 13 November 2013).

8. Cory, S.; Hsu, C.; Donn, M. Template Files for Commercial Building Stock Energy Simulations; Centre for Building Performance Research: Wellington, New Zealand, 2009.

9. Bordass, B.; Cohen, R.; Field, J. Energy Performance of Non-Domestic Buildings: Closing the Credibility Gap. In Proceedings of the Buildings Performance Congress, Frankfurt, Germany, 26-27 April 2006.

10. Urdan, T.C. Statistics in Plain English, 3rd ed.; Routledge: New York, NY, USA, 2010. 



\section{Part II:}

Productivity 



\title{
Marginal Productivity Gained Through Prefabrication: Case Studies of Building Projects in Auckland
}

\author{
Wajiha Shahzad, Jasper Mbachu and Niluka Domingo
}

Abstract: Several studies have documented benefits of prefabrication system (prefab) compared to the traditional building system (TBS). However, the documented benefits have been anecdotal or fragmented with reports of isolated case study projects. Few studies have looked at the objectively quantified benefits from statistical significance point of view and across building types in New Zealand. This study contributes to filling this knowledge gap by analyzing cost and time-savings, and productivity improvement achievable by the use of prefab in place of the TBS. Records of completion times and final contract values of 66 building projects implemented using prefab in Auckland were collected. The building types included commercial, houses, apartments, educational, and community buildings. The project details included final contract sums, completion dates, gross floor areas, and number of floors. Based on these details, the equivalent completion times and the final cost estimates for similar buildings implemented using the TBS were obtained from the Rawlinsons construction data handbook and feedback from some designers and contractors. Marginal productivity outcome for each building project was computed as the product of the cost and time-savings achieved using the prefab. Results showed that the use of prefab in place of TBS resulted in 34\% and 19\% average reductions in the completion times and costs, respectively. This also translated to overall $7 \%$ average improvement in the productivity outcomes in the building projects. Univariate ANOVA-based hypothesis test results showed that 'building type' had no significant effects on the cost and productivity improvement outcomes, but had significant effect on the time savings analyzed in the case study projects. The greatest productivity gain of $11 \%$ was achieved in house projects. These evidence-based results could guide optimized use of prefab for specific building application. The hypothesis-testing outcome provides insights on one of several potential influences on prefab improvements, which will be analyzed in subsequent research.

Reprinted from Buildings. Cite as: Shahzad, W.; Mbachu, J.; Domingo, N. Marginal Productivity Gained Through Prefabrication: Case Studies of Building Projects in Auckland. Buildings 2015, 5 , 196-208.

\section{Introduction}

Offsite manufacture or prefabrication of building components and systems has been acknowledged globally by many industry-driven commissions of enquiry as an effective solution to the several problems faced by the construction industry [1]. The technique readily supports standardization and rapid prototyping (including 3D printing/additive manufacturing technologies), which are expected to re-engineer the future of the construction industry [2].

Numerous benefits that prefab offers compared to the traditional building system (TBS) have been documented in several studies. These included better quality of work [3,4], better environmental performance and safety records [5,6], significant cost and time savings [2,7], and enhanced productivity and efficiency [4]. However, the benefits have been anecdotal or fragmented, and involved reports 
of isolated case studies [5,8]. Few studies have looked at the evidence-based and quantified benefits from statistical significance point of view and across building types. The low level of industry uptake of prefab hinges in part on the lack of quantifiable evidence to support claims about the numerous benefits credited to the technology [1]. This research contributes to filling this knowledge gap by seeking and analyzing hard data on the marginal benefits of the technology.

\subsection{Prefabrication in Context}

The Modular Building Institute [9] defines "prefabrication" as the process of manufacturing and assembling the major building components at remote offsite locations for their subsequent installation on construction site. Operationally, prefabrication is a construction innovation, which aims to take as much as possible the construction activities away from the project site to the factory to ensure better quality and safer production under controlled working conditions. This construction approach is seen as being more environmentally friendly, safer and productive than the conventional stick-built approach [10,11]. Shahzad and Mbachu [1] argue that there is increasingly little differentiation between the conventional building types and the "componentized" and the "panelized" prefabrication types. This is because conventional buildings involve some form of "componentized" and "panelized" prefab units or the other. In this context, the differentiation is made by checking the value or proportion of the prefabrication components compared to the onsite manufactured components. On this basis, a building is classified as a prefab building where the prefab component is more than $50 \%$ of the total building value, or vice versa.

\subsection{Benefits of Prefabrication Compared to Conventional Construction Methods}

Several studies have explored benefits of prefabrication technology. These benefits included reduced project cost, shorter project duration and on time delivery of projects [12], enhanced quality of construction [12,13] improved onsite health and safety [14], reduced onsite wastage and environmental impact, and reduced whole life cycle costs [1]. Lu [14] observes that prefab does not only save construction costs but also offers more reliable estimates of the upfront costs, total investment outlay and overall returns on investments. Other advantages of the modular prefabrication over and above conventional construction methods include better compliance with the Building Codes, quicker processing of the building consents/permits, and fewer building inspections [1]. In addition, Modular Building Institute [9] maintains that prefabrication optimizes the use of construction materials, resulting in less amount of waste generation.

\subsection{Issues with Prefabrication System of Construction}

Despite the benefits of prefabrication, there is still low-uptake of the technology in many countries [1]. Several studies explored the issues with the prefabrication technology in general. Some of the issues relate to the need for expensive haulage and cranage for handling large prefab components $[9,13]$, the long lead time required for ordering and supply of prefab components, and the lack of flexibility to permit bespoke designs at the design phase [15]. 
From a socio-cultural perspective, Bell [3] notes the misperceptions about the technology based on cultural issues and social stigma attached to the technology due to negative experiences in the past about the quality of prefab buildings, especially, during the post-world war reconstruction era, as well as the temporary nature and poor aesthetics of prefab buildings. Shahzad and Mbachu [1] note the building owner's penchant for bespoke designs which allows them to make changes to suite lifestyle preferences throughout the design and initial construction stages. The conventional building approach offers this flexibility to a large extent and also allows room for more proactive change management, whereas the prefab approach usually limits the extent of the owner's changes to the standard designs. If significant changes are made, especially at the construction phase, the outcomes for the prefab technology in terms of costs, speed and wastage will be less desirable when compared to the corresponding outcomes for the conventional system. Other issues include the logistic challenge (especially for projects located in traffic congested areas), onsite connection or interface problems, and the reality that construction-phase changes are bound to be made due to the variability of site conditions from the initial design assumptions. In these circumstances, the conventional building method proves to be more suitable than the prefab system.

In sum, prefab is still globally viewed as the way of the future in the construction industry. Its benefits certainly outweigh its shortcomings. The question is, why is it then that the technology suffers low industry-wide uptake? Part of the answer to this problem could be lack of empirical evidence with which to support the numerous benefits credited to the technology. To enable a choice to be made between prefab and conventional building systems, owners would want credible and quantified evidence of the marginal benefits the technology offers. Current evidence is still anecdotal or is not robust enough due to hasty conclusions being made on isolated case study results.

\subsection{Research Objectives}

The main objectives of this study were: (1) To quantify the marginal benefits of the prefab building system in terms of the cost, time and productivity improvements it can offer over and above the corresponding benefits achievable with the TBS; (2) To examine how any observed benefits may be affected by the "building type" as a fixed independent factor.

\subsection{Scope and Limitations}

The study is limited to the historical records of final contract sums and durations of 66 building projects completed in Auckland. The building types covered included commercial, houses, apartments, educational, and community buildings. The need to limit the project locations to Auckland is to minimize any bias which location as a fixed independent factor could have on the research outcomes. Case study building projects investigated under each building category comprised 33 houses (50\%), 16 commercial (24\%), 7 educational (11\%), 5 community (8\%), and 5 apartment $(8 \%)$ buildings, totalling 66 projects. Majority of the case study building projects were houses.

Proportions of the prefab systems used in the 66 case study projects were as follows: Componentized and panelized (CP) 30 (45\%), hybrid (modular and CP) 20 (30\%), modular 13 (20\%), 
and whole house $3(5 \%)$. The $\mathrm{CP}$ form of prefab therefore constituted the greatest proportion of the projects investigated.

\section{Research Methods}

Historical cost information on some completed projects provided empirical data for the study. A mixed method of archival research and case study was therefore adopted as the appropriate research method as it permitted the extraction of information from archived records [16]. This method also aligns with the research objective of obtaining multiple sources of evidence from different cases (i.e., building types) [17].

In addition, Cooper and Emory [18] recommend the use of case study research method where data samples are chosen for relevance to the breadth of the issue under investigation rather than on the basis of how well they represent the target population. Investigations were focused on exploring the potential marginal time and cost savings that could be achieved by the use of prefab system over and above the outcomes for conventional building system. With no pre-defined sampling frame for the study, final contract sums and completion times of the prefab buildings covered in the study were obtained through contacts with contracting and consulting firms in Auckland. In total archived records of 66 prefab building projects were examined. For the purpose of comparative analysis, the key historical project information required for each prefab building type was mainly the building characteristics that determined the price information in the Rawlinsons Construction Handbook [19]. Information for each of the five building types included cost at completion, duration, gross floor area (GFA) and number of floors. The need to adjust the effects that differing regimes of inflation and exchange rates had on the historical cost information was obviated by the use of percentage differences as the common denominator for comparison.

To obtain the empirical data, purposive sampling method [20] was used, since there were no databases from which to sample the projects. Through the assistance of the Registered Master Builders Federation of New Zealand, contractors who were willing to release records of their past projects for the purposes of the research were contacted. They were promised anonymity, assuring that the data would be used solely for academic research purposes with no revealing details about their projects, their companies or their clients. The efforts in this direction yielded 66 prefab project records. The equivalent completion times and the final cost estimates for similar buildings implemented using the TBS were obtained from the Rawlinsons construction data handbook as well as feedback from some designers and contractors.

\subsection{Method of Data Analysis}

The data required for the first objective were the final costs and completion times of prefab buildings under each building category. The data analysis involved computing for the following parameters for the $j$ th project within a set for a particular building type $i$ : 


\subsubsection{Marginal Cost Saving $\left(\mathrm{MCS}_{i j}\right)$}

This was computed as the difference between the final cost of the prefab building and the corresponding cost for a similar building erected using the traditional system, expressed as a percentage of the latter. Equation (1) provides the expression for the $\mathrm{MCS}_{i j}$ :

$$
\operatorname{MCS}_{i j}=\left(\frac{C_{\mathrm{TRAD} i j}-C_{\mathrm{PREFAB} i j}}{C_{\mathrm{TRAD} i j}}\right)
$$

where $\mathrm{MCS}_{i j}$ is marginal cost saving (MCS) achieved in the $j$ th project within the set of buildings for the particular building type $i$; $C_{\text {PREFAB } i j}$ is final cost of the $j$ th prefab building; $C_{\text {TRAD } i j}$ is corresponding final cost of a similar building completed using the traditional building system.

The rationale for computing the productivity improvement as a product of marginal time and cost savings draws upon two streams of thoughts:

First, in the construction industry context, productivity performance depends largely on the cost and schedule performance [21]. This strategic perspective of the concept of productivity differs to some extent from the economist's perspective of productivity which is basely solely on output versus input resource ratio, featuring variants, such as labour, capital and multi-factor productivity measures. Mbachu and Shahzad [7] clearly made this distinction. The mathematical expression for an integrated productivity measurement based on the two key parameters of cost and time savings draws from the fact that productivity is directly proportional to the cost and schedule performance, i.e.:

$$
\begin{gathered}
P \propto\left(S_{p}, C_{P}\right) \\
P=\beta\left(S_{P} \times C_{P}\right)
\end{gathered}
$$

where $P$ is the productivity performance achieved in a project; $\beta$ is an empirically determinable constant of proportionality that depends on the dynamics of the operational environment. The constant could be taken as unity (i.e., value of 1) for projects executed under normal operating conditions as assumed in the study; $S_{p}$ is the schedule performance computed using a modified form of Equation (1); $C_{p}$ is the cost performance computed using Equation (1).

\subsubsection{Average Marginal Cost Saving $\left(\mathrm{AMCS}_{i}\right)$}

This was computed as the average of the MCS for all the case study projects for a particular building type. Equation (4) provides the expression for evaluating the average marginal cost saving $\left(\mathrm{AMCS}_{i}\right)$ :

$$
\mathrm{AMCS}_{i}=\left(\frac{\sum_{j=1}^{n} \mathrm{MCS}_{i j}}{n}\right)
$$

where $\mathrm{AMCS}_{i}$ is average marginal cost savings achieved in all the $n$ case study buildings for the building type $i$. 
2.1.3. Marginal Time Saving $\left(\mathrm{MTS}_{i j}\right)$ and Average Marginal Time Saving $\left(\operatorname{AMTS}_{i}\right)$

These were computed as for the $\mathrm{MCS}_{i j}$ and $\mathrm{AMCS}_{i}$ in Equations (1) and (4) above, respectively.

\subsubsection{Productivity Improvement $\left(\mathrm{PI}_{i}\right)$}

This was computed on two levels: At the level of the individual building types, it was computed as the product of the marginal cost and time savings achieved in each case study project $j$ within the set for the building type $i$, i.e.:

$$
\mathrm{PI}_{i j}=\mathrm{MCS}_{i j} \times \operatorname{MTS}_{i j}
$$

where $\mathrm{PI}_{i j}$ is productivity improvement achieved in the $j$ th project for the building type $i$; $\mathrm{MCS}_{i j}$ is marginal cost saving achieved in the $j$ th project for the building type $i$; $\mathrm{MTS}_{i j}$ is marginal time savings achieved in the $j$ th project for the building type $i$.

At the level of the building type $i$, the productivity improvement was computed as the product of the average cost and time savings achieved in the case study projects for a particular building type $i$ :

$$
\mathrm{PI}_{i}=\mathrm{AMCS}_{i} \times \operatorname{AMTS}_{i}
$$

where $\mathrm{PI}_{i}$ is productivity improvement achieved in all the $n$ case study buildings for the building type $i$; $\mathrm{AMCS}_{i}$ is average marginal cost savings achieved in all the $n$ case study buildings for the building type $i$ : AMTS $_{i}$ is average marginal time savings achieved in all the $n$ case study buildings for the building type $i$.

\subsubsection{Average Productivity Improvement $\left(\mathrm{API}_{\mathrm{av}}\right)$}

This was computed as the average of the productivity improvements achieved across all $\mathrm{m}$ building types:

$$
\mathrm{API}_{\mathrm{av}}=\left(\frac{\sum_{i=1}^{m} P I_{i}}{m}\right)
$$

where $\mathrm{API}_{\mathrm{av}}$ is Average Productivity Improvement achieved in all the $m$ building types.

\subsection{Hypothesis Testing}

The statistical tests of significance, which informed the second objective of the study involved a null hypothesis that "building type" as a fixed independent factor did not have significant effect on any marginal benefits achieved by the use of prefab system in the case study projects. The alternative hypothesis assumed otherwise. The hypothesis testing required a single factor analysis of variance (ANOVA) given the single independent factor involved (i.e., "building type") and the interval scale of the empirical data [22]. The hypothesis testing was conducted separately for the 
observed cost, time and productivity marginal benefits. Typical procedure for the test involving time savings is defined as follows:

H0T: There is no significant difference in the average time-savings achieved in the five building groups. If this were true, it would mean that "building type" would have no significant effect on the time-savings achieved by the use of prefab in place of conventional method.

The alternative hypothesis was formulated as follows:

HaT: The differences in the average time-savings achieved in the five building groups are significant.

Acceptance condition: Accept H0T if the $p$-value or confidence level of the single factor ANOVA test is higher than the 0.05 alpha value for the test.

Rejection condition: Reject H0T if the $p$-value is equal to or lower than the alpha value for the test; accept the alternative hypothesis instead.

The above hypothesis tests were replicated for the cost and productivity improvements.

\section{Results and Discussions}

Results relating to the two objectives of the study are presented and discussed in the following subsections.

\subsection{Cost, Time, and Productivity Improvement Achieved by Use of Prefab}

The first research objective was to quantify the marginal benefits of the prefab building system in terms of the cost, time and productivity improvement it delivers over and above the outcomes achievable with the traditional building system (TBS). Table 1 presents typical example of the marginal benefit analysis for the office/commercial building projects. This building category comprised 18 case study projects. Similar computations were done for the other 4 building categories. The results were summarized for all the buildings types and plotted in Figure 1 for better visual appreciation.

Figure 1 shows that the marginal cost saving delivered by the prefab system over the TBS was $19 \%$ on average. The highest cost saving (24\%) was achieved in the community building projects. Perhaps, the relatively simpler design nature of community buildings might have contributed to this result.

On the other hand, the average marginal time saving achieved by the prefab system in the case study projects was $34 \%$. The highest time saving (i.e., 50\%) was achieved in the house projects. This result could be due to majority of the houses being developed off standard plans provided by group home builders. Such houses lend readily to standard components, which make for faster manufacture and installation, thereby enhancing speed of construction. In addition, in terms of costs, this method of housing procurement has been found to be $15 \%$ cheaper than houses that were of one-off designs [23]. The overall marginal productivity improvement offered by prefab system was $7 \%$, with the house projects having the highest (11\%) productivity gain. This result might suggest that prefab could provide the most effective approach to delivering the 400,000 houses 
planned to be built over the next 3 years under the Auckland Housing Strategy [24], as well as the $\$ 30$ billion Canterbury rebuild [25].

Overall, the findings of this study in relation to the prefab marginal cost, time and productivity improvements were in agreement with conclusions reached in previous studies overseas. For instance, Egan [26] argues that the use of prefab system has many benefits including speed of construction, lower cost, reduced need for skilled labour and achievement of zero defects, all of which could have significant impact (about 30\%-40\% improvement ) on on-site productivity level. However, some New Zealand studies did not concur with overseas results on superior economic benefits of prefab system. For instance, Burgess et al. [27] found that prefabricated buildings did not yield better economic choice than onsite construction in a case study house investigated in their work. This can be associated to small size of the New Zealand market and its inability to exploit the scale efficiencies offered by mechanized bulk production of similar products. It was not clear whether the New Zealand results were due to inadequate sample size-with the study being based on a few case study projects.

Table 1. Prefab marginal benefits (typical analysis for commercial building projects).

\begin{tabular}{|c|c|c|c|c|c|c|c|c|c|c|}
\hline Project & $\begin{array}{l}\text { GFA } \\
\left(\mathrm{m}^{2}\right)\end{array}$ & Storey & $\begin{array}{c}\text { Date } \\
\text { Completed }\end{array}$ & $\begin{array}{c}\text { Prefab Cost } \\
\text { (\$) }\end{array}$ & $\begin{array}{c}\text { * Equiv TBS } \\
\text { Cost }(\$)\end{array}$ & $\begin{array}{l}\text { \% Cost } \\
\text { saving a }\end{array}$ & $\begin{array}{l}* * \text { Prefab } \\
\text { dur (wks) }\end{array}$ & $\begin{array}{c}* * * \text { Equi TBS } \\
\text { dur (wks) }\end{array}$ & $\begin{array}{l}\text { \% Time } \\
\text { Saving } \\
\end{array}$ & $\begin{array}{c}\text { Productivity } \\
\text { Improvt }^{\mathrm{c}}\end{array}$ \\
\hline 1 & 5,900 & 6 & 5 October & $6,000,000$ & $14,553,000$ & 58.77 & 72 & 84 & 14.29 & 8.40 \\
\hline 3 & 1,290 & 2 & 8 August & $1,500,000$ & $3,160,500$ & 52.54 & 24 & 52 & 53.85 & 28.29 \\
\hline 4 & 2,850 & 2 & 8 April & $5,500,000$ & $6,982,500$ & 21.23 & 32 & 64 & 50.00 & 10.62 \\
\hline 6 & 5,400 & 1 and 2 & 13 August & $7,000,000$ & $10,665,000$ & 34.36 & 48 & 72 & 33.33 & 11.45 \\
\hline 7 & 4,082 & 1 & 13 November & $7,000,000$ & $8,061,950$ & 13.17 & 32 & 66 & 51.52 & 6.79 \\
\hline 8 & 1,001 & 1 & 12 August & $2,000,000$ & $3,461,000$ & 42.21 & 36 & 50 & 28.00 & 11.82 \\
\hline 9 & 9,000 & 1 & $11 \mathrm{March}$ & $8,000,000$ & $14,085,000$ & 43.20 & 32 & 84 & 61.90 & 26.74 \\
\hline 10 & 150 & 2 & 9 January & 160,000 & 189,000 & 15.34 & 4 & 10 & 60.00 & 9.21 \\
\hline 11 & 100 & 1 & 13 March & 220,000 & 243,000 & 9.47 & 16 & 18 & 11.11 & 1.05 \\
\hline 12 & 10,000 & 8 & 12 October & $30,000,000$ & $35,400,000$ & 15.25 & 80 & 100 & 20.00 & 3.05 \\
\hline 13 & 2,100 & 2 and 3 & $12 \mathrm{March}$ & $3,200,000$ & $3,831,100$ & 16.47 & 30 & 52 & 42.31 & 6.97 \\
\hline 14 & 10,000 & 4 & 8 July & $19,000,000$ & $20,488,000$ & 7.26 & 60 & 90 & 33.33 & 2.42 \\
\hline 15 & 23,000 & 6 & 13 February & $105,000,000$ & $112,125,000$ & 6.35 & 112 & 130 & 13.85 & 0.88 \\
\hline 16 & 5,240 & 4 & 8 June & $9,600,000$ & $12,602,200$ & 23.82 & 40 & 76 & 47.37 & 11.28 \\
\hline 17 & 2,100 & 1 & 12 October & $6,500,000$ & $6,615,000$ & 1.74 & 40 & 60 & 33.33 & 0.58 \\
\hline 18 & 1,547 & 5 & 13 September & $7,800,000$ & $7,967,050$ & 2.10 & 44 & 64 & 31.25 & 0.66 \\
\hline - & - & - & - & - & Averages ${ }^{\mathrm{d}, \mathrm{e}, \mathrm{f}}$ & 22.71 & - & - & 36.59 & 8.76 \\
\hline
\end{tabular}

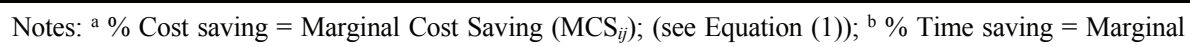
Time Saving ( $\mathrm{MTS}_{i j}$ ); (modified Equation (1)); ${ }^{\mathrm{c}}$ Productivity improvement $\left(\mathrm{PI}_{i j}\right.$ ) (see Equation (5)); ${ }^{\mathrm{d}}$ Average $\%$ cost saving = Average Marginal Cost Saving $\left(\mathrm{AMCS}_{i}\right)$; (see Equation (4)); ${ }^{\mathrm{e}}$ Average \% time saving $=$ Average Marginal Time Saving $\left(\operatorname{AMTS}_{i j}\right)$; (modified Equation (4)); ${ }^{\mathrm{f}}$ Average productivity improvement $\left(\mathrm{API}_{a v}\right)$ (see Equation (7)). * Equivalent TBS cost in NZ\$; ** Prefab duration in weeks; $* * *$ Equivalent TBS duration in weeks. 


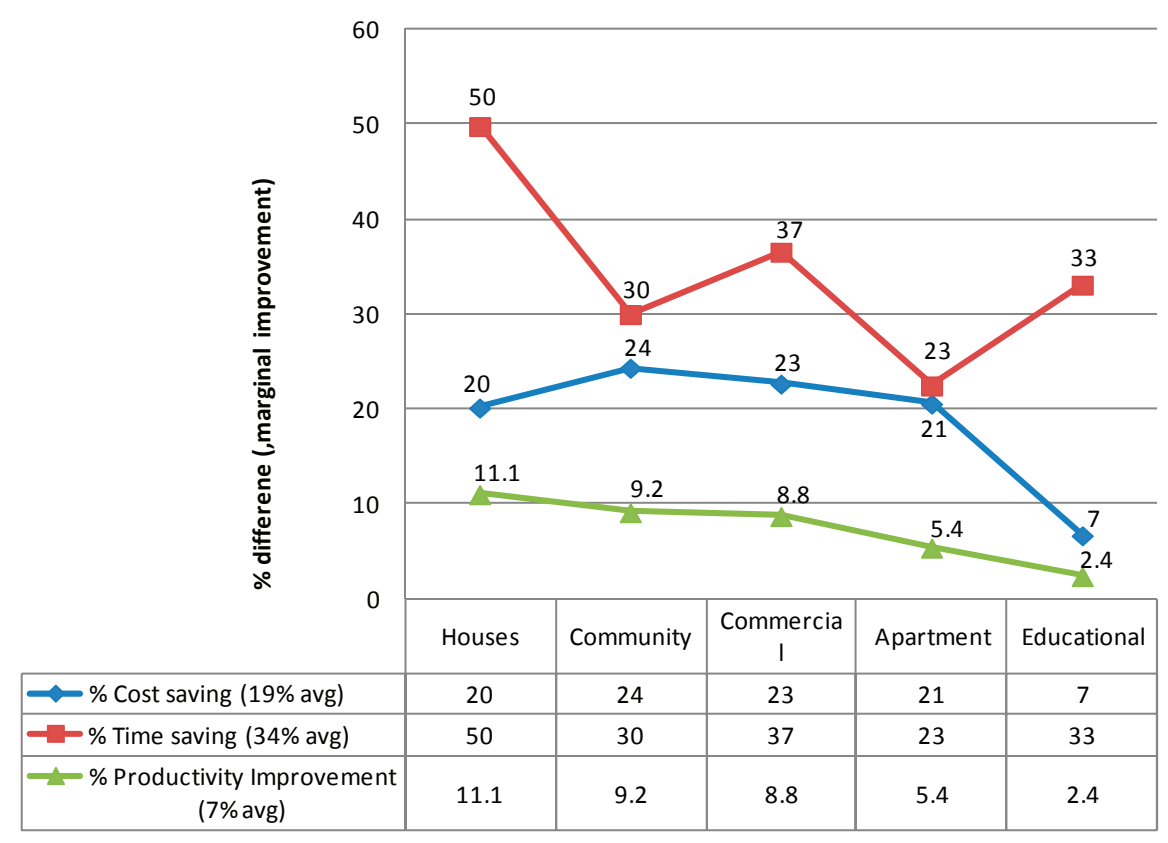

Figure 1. Time, cost and productivity improvement achieved by the use of prefab in place of traditional building system in the case study projects.

\subsection{Influence of Building Type on the Analyzed Prefab Marginal Benefits}

The second research objective was to examine how any observed benefits offered by prefab building system could be affected by "building type" as a fixed independent factor. As discussed under the methodology section, achieving this objective required testing for the null hypothesis formulated to imply that "building type" as a fixed independent factor did not have significant effect on any marginal benefits achieved by the use of prefab system in the case study projects. Results of the single factor ANOVA employed in the test are presented in Table 2. The tests were carried out separately for prefab marginal productivity improvement, cost saving and time saving.

Table 2 shows that the $p$-value analyzed in the null hypothesis test for the productivity improvement was greater than the alpha value of 0.05 used in the test (i.e., $0.09>0.05$ ). Additionally, the F-ratio of 2.11 was less than the $\chi^{2} F$-critical value of 2.52 . On account of these results, the null hypothesis could not be rejected. It was therefore concluded that "building type" had no significant influence on the marginal productivity improvement benefits delivered by the prefab system.

Similar conclusion was also reached for the marginal cost saving. However, the result was different for the time saving: A $p$-value of 0.005 which is less than the alpha value of 0.05 meant that building type impacted significantly on the observed prefab marginal time saving. This suggests that the use of prefab system may result in shorter completion times of buildings than the TBS depending on the building type, whereas the cost and productivity improvement benefits are not 
influenced by building type, and are likely to be consistent across all building types. Thus, claims about the superior benefits of prefab can only be generalised in respect of cost and productivity improvements across all building types, whereas claims about time savings should be cautiously applied to specific building types. There is no empirical evidence in this study to conclude that the technology is superior to the traditional building system in terms of time savings across all building types. This latter finding contrasts with conclusions reached in a number of New Zealand and overseas studies which seemed to generalize the benefits across all building types. Burgess et al. [27] — Citing Kaufmann and Remick [28], Atkin and Wing [29], and Bell [3] — Stated that "time savings for prefabricated construction for residential applications is between $30 \%$ and $60 \%$ " (p. 25). The qualified statement (i.e., "residential applications") made by Burgess et al. [27] is therefore worthy of emulation when it comes to reporting on the time saving benefits of prefab system. The question that may be worthy of further investigation is why the superior time saving benefits of the technology may not be consistent across all building types. A possible clue might be found in the observations of Shahzad and Mbachu [1] that there is increasingly little differentiation between the conventional building types and the "componentized" and the "panelized" prefabrication types. This is because conventional buildings involve some form of "componentized" and "panelized" prefab units or the other. This is true for most non-residential building constructions. On the other hand, clear differentiation exists in the application of the technology to residential buildings - a clear departure from the traditional stick-built system which dominates residential construction. The large proportion of residential prefab projects (about 50\%) might have influenced the hypothesis test result in this regard. However, then, why were the cost and productivity improvement benefits not also influenced by the larger proportion of residential building project samples? These are issues to be resolved in future research.

Table 2. Univariate ANOVA analysis results for hypothesis testing.

\begin{tabular}{|c|c|c|c|c|c|c|c|c|}
\hline Dimension & Variation Source & ${ }^{\text {a }} \mathbf{S S}$ & ${ }^{\mathrm{b}} \mathbf{D F}$ & ${ }^{\mathrm{c}} \mathbf{M S}$ & ${ }^{d} F$-ratio & ${ }^{\mathrm{e}} p$-value & ${ }^{\mathrm{f}} F$-crit & Result \\
\hline \multirow{3}{*}{$\begin{array}{l}\% \text { productivity } \\
\text { improvement }\end{array}$} & Between groups & 505.7 & 4 & 126.4 & 2.11 & 0.09 & 2.52 & Do not reject $\mathrm{H} 0(p$-value $>0.05)$ \\
\hline & Within groups & $3,651.6$ & 61 & 59.9 & - & - & - & - \\
\hline & Total & $4,157.2$ & 65 & - & - & - & - & - \\
\hline \multirow{3}{*}{$\%$ cost saving } & Between groups & $1,441.0$ & 4 & 360.3 & 1.50 & 0.21 & 2.52 & Do not reject $\mathrm{H} 0(p$-value $>0.05)$ \\
\hline & Within groups & $14,618.8$ & 61 & 239.7 & - & - & - & - \\
\hline & Total & $16,059.8$ & 65 & - & - & - & - & - \\
\hline \multirow{3}{*}{$\%$ time saving } & Between groups & $5,617.3$ & 4 & $1,404.3$ & 5.81 & 0.0005 & 2.52 & Do not accept $\mathrm{H} 0$ ( $p$-value $<0.05 \mathrm{a})$ \\
\hline & Within groups & $14,747.0$ & 61 & 241.8 & - & - & - & - \\
\hline & Total & $20,364.3$ & 65 & - & - & - & - & - \\
\hline
\end{tabular}

Notes: a SS (Sum of squares) = sum of squares of variations of data points from the means for the between and within group; ${ }^{\mathbf{b}} \mathrm{DF}$ (degree of freedom) = sum of degrees of freedom for each group computed for each group as number of data points -1 ); ${ }^{\mathbf{c}}$ MS (mean squares) $=\mathrm{SS} / \mathrm{DF} ;{ }^{\mathbf{d}} F$-ratio $=\chi^{2}$ test statistic $=$ MS (between groups)/MS (within groups); ${ }^{\mathrm{e}} p$-value $=$ probability value associated with the $F$-ratio; ${ }^{\mathrm{f}} F$-crit $=$ critical $F$-ratio value from statistics tables corresponding to the alpha level of test (i.e., 0.05). 


\section{Conclusions}

This study has quantified the marginal benefits of the prefab building system in terms of cost, time and productivity improvements it can offer over and above corresponding benefits achievable with the traditional building system (TBS). Potential influence of building types on the observed benefits was also examined. Results showed that the marginal cost saving delivered by the prefab system over the TBS was $19 \%$ on average. The highest cost saving (24\%) was achieved in community building projects. On the other hand, average marginal time saving achieved was $34 \%$, with the highest time saving of 50\% achieved in the house projects. The overall marginal productivity improvement offered by prefab system was $7 \%$, with house projects having the highest productivity gain of $11 \%$. This result might suggest that prefab could provide the most effective approach to delivering the 400,000 houses planned to be built under the Auckland Housing Strategy over the next three years [24], as well as the $\$ 30$ billion Canterbury rebuild [25].

Results of ANOVA tests on how the observed benefits offered by prefab building system could be affected by "building type" were mixed. Whereas cost and productivity improvement benefits offered by the system were consistent across building types, time saving benefits were influenced by the building type. Empirical evidence therefore supports the generalization of the cost and time benefits across building types. This is not applicable to time savings, which require being reported only in relation to specific building types.

Influences of other fixed factors such as location, project size/complexity, procurement and contract strategies, type of prefabrication and the degree of standardization or replication are key limitations of the study which are recommended for further investigations. It should also be noted that variability issues could be associated with the limited dataset of 66 projects used in the study; these could raise some reliability concerns if the findings were to be generalized beyond the scope of this study.

\section{Acknowledgments}

This research is funded by Building Research Association of New Zealand (BRANZ) building research levy through the Postgraduate Research Scholarship for which the authors are grateful. In addition to this, the cooperation of construction companies and project managers in providing the requisite data is highly acknowledged.

\section{Author Contributions}

The research was part of a thesis undertaken by the author for correspondence Wajiha Shahzad. It was supervised by Jasper Mbachu (principal supervisor) and Niluka Domingo (assistant supervisor).

\section{Conflicts of Interest}

The authors declare no conflict of interest. 


\section{References}

1. Shahzad, W.M.; Mbachu, J. Productivity Enhancement of Construction Industry Using Prefabrication: Impact Levels of the Underlying Constraints and Improvement Measures; Lambert Academic Publishing: Saarbrücken, Germany, 2012.

2. National Research Council. Advancing the Competitiveness and Efficiency of the U.S. Construction Industry; The National Academies Press: Washigton, DC, USA, 2009.

3. Bell, P. Kiwi Prefab: Prefabricated Housing in New Zealand: An Historical and Contemporary Overview with Recommendations for the Future. Master's Thesis, Victoria University of Wellington, Wellington, New Zealand, 30 June 2009.

4. Department of Building and Housing (DBH). Report of the Building and Construction Sector Productivity Taskforce; DBH: Wellingtion, New Zealand, 2009.

5. Cooperative Research Centre (CRC). Off-Site Manufacture in Australia: Current State and Future Directions; CRC: Canberra, Australia, 2007.

6. Jaillon, L.; Poon, C.S. Design issues of using prefabrication in Hong Kong building construction. Constr. Manag. Econ. 2010, 28, 1025-1042.

7. Mbachu, J.; Shahzad, W.M. A Holistic Understanding of the Concept and Measurement of Productivity in the Construction Industry: A New Zealand Perspective. In Proceedings of the WABER 2012 International Conference, Abuja, Nigeria, 24-26 July 2012; pp. 87-98.

8. Davis, N. Construction Sector Productivity: Scoping Report; Department of Building and Housing: Wellington, New Zealand, 2007.

9. Modular Building Institute (MBI). Improving Construction Efficiency \& Productivity with Modular Construction Charlottesville; MBI: Charlottesville, VA, USA, 2010.

10. Arif, M.; Egbu, C. Making a case for offsite costruction in China. Eng. Constr. Arcitectural Manag. 2010, 17, 536-548.

11. Azman, M.N.A.; Ahamad, M.S.S.; MAjid, T.A.; Hanafi, M.H. The common approach in off-site construction industry. Aust. J. Basic Appl. Sci. 2010, 4, 4478-4482.

12. Lusby-Taylor, P.M.S.; Ainger, C.; Ogden, R. Design and Modern Methods of Construction; The Commission for Architecture and the Built Environment (CABE): London, UK, 2004.

13. Gibb, A. Off-Site Fabrication; Whittles Publishing: Scotland, UK, 1999.

14. Lu, N. The Current Use of Offsite Construction Techniques in the United States Construction Industry; Construction Research Congress: Seatle, WA, USA, 2009.

15. Bradsher, K. An Ambitious Construction Schedule Using Prefab Modules, with Questions. N. Y. Times 2013. Available online: http://www.nytimes.com/2013/08/28/business/global/anambitious-construction-schedule-using-prefab-parts.html (accessed on 6 February 2015).

16. Cooper, D.R.; Schindler, P.S. Business Research Method; McGraw Hill/Irwin: New York, NY, USA, 2006.

17. Mbachu, J.; Nkado, R. Conceptual framework for assessment of client needs and satisfaction in the building development process. Constr. Manag. Econ. 2006, 24, 31-44.

18. Cooper, D.R.; Emory, C.W. Business Research Methods; Irwin Inc.: Chicago, IL, USA, 1995. 
19. Giddens, C. Rawlinsons New Zealand Construction Handbook, 28th ed.; Rawlinsons Limited: Auckland, New Zealand, 2014.

20. Saunders, M.; Lewis, P.; Thornhill, A. Research Methods for Business Students; Pitman Publishing: London, UK, 1997.

21. Takim, R.; Akintoye, A. Performance Indicators for Successful Construction Project Performance. In the Proceedings of 18th Annual ARCOM Conference, Northumbria, UK, 2-4 September 2002; Greenwood, D, Ed.; Association of Researchers in Construction Management: Edinburgh, UK, 2002; Volume 2, pp. 545-555.

22. Statistical Product and Services Solution (SPSS), version 21.0; IBM: Armonk, NY, USA, 2012.

23. Building Research Association of New Zealand (BRANZ). BRANZ Response to Ministry of Business, Innovation and Employment: Residential Construction Sector Market Study; BRANZ: Wellington, New Zealand, 2013.

24. 40,000 Homes to Be Built Under Auckland Housing Strategy. OneNews, 13 June 2013. Available online: http://tvnz.co.nz/national-news/40-000-homes-built-under-auckland-housing-strategy5433767 (accessed on 5 February 2015).

25. Ministry of Business, Innovation and Employment (MBIE). New Zealand Sectors Report: Featured Sector-Construction; MBIE: Wellington, New Zealand, 2013.

26. Egan, J. Rethinking Construction; Secretary of State for the Environment, Transport and the Regions: London, UK, 1998.

27. Burgess, J.C.; Buckett, N.R.; Page, I.C. Prefabrication Impacts in the New Zealand Construction Industry; Building Research Assocition of New Zealand (BRANZ): Wellington, New Zealand, 2013.

28. Kaufmann, M.; Remick, C. PreFab Green; Gibbs Smith: Santa Barbara, CA, USA, 2009.

29. Atkin, B.; Wing, R. Future Home-Manufactured Housing for Europe. In Proceedings of the 17th International Symposium on Automation and Robotics in Construction, Madrid, Spain, 22-24 September 1999. 


\title{
Building Information Modelling for Smart Built Environments
}

\author{
Jianchao Zhang, Boon-Chong Seet and Tek Tjing Lie
}

\begin{abstract}
Building information modelling (BIM) provides architectural 3D visualization and a standardized way to share and exchange building information. Recently, there has been an increasing interest in using BIM, not only for design and construction, but also the post-construction management of the built facility. With the emergence of smart built environment (SBE) technology, which embeds most spaces with smart objects to enhance the building's efficiency, security and comfort of its occupants, there is a need to understand and address the challenges BIM faces in the design, construction and management of future smart buildings. In this paper, we investigate how BIM can contribute to the development of SBE. Since BIM is designed to host information of the building throughout its life cycle, our investigation has covered phases from architecture design to facility management. Firstly, we extend BIM for the design phase to provide material/device profiling and the information exchange interface for various smart objects. Next, we propose a three-layer verification framework to assist BIM users in identifying possible defects in their SBE design. For the post-construction phase, we have designed a facility management tool to provide advanced energy management of smart grid-connected SBEs, where smart objects, as well as distributed energy resources (DERs) are deployed.
\end{abstract}

Reprinted from Buildings. Cite as: Zhang, J.; Seet, B.-C.; Lie, T.T. Building Information Modelling for Smart Built Environments. Buildings 2015, 5, 100-115.

\section{Introduction}

Computer-aided design (CAD) techniques have been in use by the construction industry since the early 1980s. The next paradigm shift was the introduction of building information modelling (BIM) in the field of architecture, engineering and construction (AEC) in the mid-1990s [1].

BIM is a methodology enabled by a set of software tools and processes for facilitating the creation and use of the digital representation of the physical and functional characteristics of a facility [2]. In terms of software, BIM introduces exchangeable information formats, i.e., International Foundation Classes (IFC), for modelling and visualizing building entities in 3D. In terms of processes, BIM facilitates the conveyance of building information from the design phase throughout the building life cycle, supporting cost management, construction management and facility management [3].

Thus, research on BIM as a methodology has been focused on the following two areas: (i) development of software tools and techniques for creating and evaluating new BIM artefacts that arise as building designs and technologies evolve; and (ii) the application and usage of BIM processes across the life-cycle of a building from pre-construction design to post-construction facility management. 
Recent rapid advances in information and communication technologies (ICT) have led to their pervasive use across industry sectors, including building construction. An upcoming and important aspect of ICT use that we anticipate to take a central stage is the construction and management of emerging smart built environments (SBEs). Here, "smart built environment" refers to a built environment that has been embedded with smart objects, such as sensors and actuators, with computing and communication capabilities, making the environment sufficiently 'smart' to interact intelligently with and support their human users in their day-to-day activities [4].

In a smart building, the human users refer not only to the building occupants, but also to the building's owners/managers. The former are typically concerned with how a smart building could improve personal safety, comfort and productivity, while the latter are more concerned with how a smart building could better support their operation and management of the building. In addition, as buildings are a major source of energy consumption, accounting for $40 \%$ of primary energy consumption in most countries [5], SBE will be expected to harness its new technological capabilities to achieve an unprecedented level of energy efficiency.

Therefore, constructing SBEs can have a set of requirements and procedures not defined or typically considered in traditional construction settings. Although there has been research conducted on various aspects of SBEs, very little attention has been focused on the role and application of BIM in the pre- and post-construction processes of SBEs.

Therefore, in this paper, we investigate how BIM can contribute to the pre-construction design and post-construction management phases of SBE. The rest of the paper is organized as follow: in Section 2, we review related works. In Section 3, we introduce the general challenges and the opportunities for BIM in SBE. In Section 4, BIM is first extended in the building design phase to provide material/device profiling and an information exchange interface for sensors, smart meters and DERs. In Section 5 , a facility management tool is developed to provide advanced energy management functions based on BIM produced in the previous phase. Finally, Section 6 concludes the paper.

\section{Literature Review}

In this section, we review the state-of-the-art on the design and management of smart built environments, as well as related BIM applications.

\subsection{Design and Management of $S B E$}

The design and management of SBE generally focus on two aspects: service and sensing. The service refers to that provided by SBE to support users in their daily lives. The authors in [6] discuss the service life cycle and design principles for a management framework to effectively manage autonomous and adaptive services in smart spaces. In [7], a smart shadow system was proposed to provide home users with real-world services, defined as services that affect the real world by changing the user's environmental factors, such as air conditioning and lighting, and to dynamically detect and resolve service conflicts.

On the other hand, some design approaches consider sensing as one essential component of SBE. The authors in [8] utilize a logic-based modelling language TCOZ (Timed Communicating Object 
Z) to describe constraints of the sensor imposed by its environmental conditions or relations with other sensors and its sensing pattern, such as periodic or conditional sensing. The sensor-based model was then applied to the design of smart spaces. In [9], ECA (event, condition, action) rules are introduced for describing home-based sensor-driven services, which can often cause a chain reaction, i.e., one service may generate an outcome that automatically triggers another service. Therefore, a method is proposed to detect such service chains and the possible conflicts among these services.

\subsection{Related BIM Applications}

In recent years, researchers have also explored how SBE design and post-construction management could benefit from harnessing BIM information and capabilities. In [10], an indoor wireless sensor network (WSN) is designed with BIM data that provides a detailed description of the building environment, which is required for accurate predictions of signal propagation and, therefore, link quality between sensor nodes in the building. In [11], a three-dimensional smart space design framework was proposed in which space (e.g., furniture, walls, floors, etc.) was viewed as one dimension along with technologies (for ubiquitous computing) and living (e.g., safety, health and sustainability requirements) as the other two dimensions. Bhatt et al. [12] propose an ontology-based spatial-terminological inference approach to validate work-in-progress designs of smart environments. The approach checks the design for compliance with the spatial and functional constraints of environment entities based on available architecture data in IFC format.

While not specifically for smart built environments, it is also shown in [13] that BIM can benefit post-construction facility management, such as locating components, checking maintainability and creating digital assets through its powerful visualization, analysis and control capabilities. There are a number of existing works on integrating BIM with real-time information. For example, the Autodesk research group integrated BIM with sensors and meters to provide 3D visualization of building performance and life-cycle operation [14]. The Virtual Real-time Information System (VRIS) combines an Onuma cloud-based BIM tool [15] with a real-time sensor engine called the Virtual Real-time Operating Centre (vROC) to provide building management functions [16]. However, when it comes to energy management, to the best of our knowledge, there are no existing BIM-based solutions in smart built environments which are smart grid [17] ready.

\section{Utilizing BIM in Smart Built Environments}

As mentioned before, a variety of smart objects will be ubiquitously and transparently installed in SBE to perform actions, such as sensing and control. These smart objects may interact with each other and with the environment and the environment users. The communication can be carried by either wireless (e.g., ZigBee, $802.11 \mathrm{a} / \mathrm{b} / \mathrm{g} / \mathrm{n}$ ) or wired (e.g., Ethernet, power line) information networks.

\subsection{General Challenges}

A number of questions arise when considering the life cycle of a smart built environment. First is how the smart objects are embedded in the environment. From the aspect of sensors, the physical location and the surrounding settings can significantly affect their ability to carry out specific tasks, 
e.g., ambient light or occupancy sensing. From the aspect of the information network, whether a wireless sensor network (WSN) or wired Ethernet is deployed, it should be designed to offer the smart objects an excellent level of communication.

Second is how the smart objects interact with the environment. The smart objects are situated in a specific surrounding to carry out their tasks and often require the input of space data, such as building floor plans. Furthermore, in carrying their tasks, the confluence of the actions by different smart objects may involve affecting a common set of environment factors, the impact of which should be studied and understood within a spatial context. Last, but not least, when conducting a building performance analysis, such as an energy efficiency analysis of the SBE, not only the information from sensors/meters is vital, but the building architectural/geometry data are also indispensable.

Third is the maintenance of such smart objects in a building's post-construction phase. In building management, it is common for facility managers to complain about either incomplete or inaccurate (not up-to-date) documentation [18]. To maintain a smart built environment that is more complicated than traditional structures, more building design and construction data will need to be documented and conveyed to the facility managers. In the event that the company that designed and constructed the building is no longer in business, building management can continue to function properly provided that the documented information is both comprehensive and reliable.

\subsection{Introducing BIM in Smart Built Environments}

BIM hosts the collaborative architectural information and provides the semantic knowledge of the building. With the emergence of smart built environment technology, BIM should be further developed to be capable of seamlessly integrating smart objects in building design, verifying the SBE design and feeding smart objects with relevant building-related information.

Designing SBE with BIM is both advantageous and convenient. Firstly, designers of SBE can utilize the building knowledge of BIM for planning the layouts of sensors, tags, actuators and meters. The performance of smart objects can be verified against known constraints and their layouts optimized for the best functional performance. Secondly, BIM also serves as a data repository for the physical information of smart objects. For maintenance and asset tracking in the building post-construction phase, the hardware information of smart objects can be recorded, and their installation locations can be documented and visualized in 3D.

On the other hand, BIM provides a perfect ontology database for SBE. Smart objects may be designed and manufactured by different vendors. The data they provide may vary in structure, and they may communicate using different protocols. Middleware is a popular approach to addressing such issues with heterogeneous smart objects [19]. With the introduction of BIM, each smart object can be profiled through its information exchange interface. Because BIM is standard compliant, the middleware can extract data formats of smart objects and other building information for viewing as an ontology database.

\subsection{Energy Management in Smart Built Environments}

Energy management is an important part of facility management. In SBEs, energy management can be enhanced through smart objects, such as temperature, occupancy and ambient light sensors, 
that provide data for estimating the building's energy requirements, understanding the building's energy usage patterns and decision-making by building control systems to achieve a balance between a building's energy efficiency and the comfort level of its occupants.

As awareness of energy efficiency grows within the building industry, the trend of deploying various sensors in the buildings will only become more common in energy management practice. Furthermore, with the introduction of a smart grid, buildings have an active role in the power system, as they exchange electrical power information with the power grid via their smart meters [20]. Sub-metering systems are also important to achieve energy awareness for building management, as they can provide high-resolution monitoring data down to the individual appliance level.

Another significant aspect that challenges energy management today is the penetration of DERs, such as photovoltaic (PV) panels and micro wind turbines. With on-site DERs, the role of building changes from a pure energy consumer to both an energy producer and consumer (or "prosumer") [21]. Correspondingly, the role of SBE is also extended to include the tasks of performing energy generation forecast, load scheduling, storing and feeding energy back to the grid. The monitoring and control of DERs is therefore vital for energy management in SBEs.

In essence, smart meters and DERs can be viewed as real-time information objects, which are a type of smart object that can be embedded in the BIM design of SBE, and the energy management system can benefit from the profiling capability of BIM, as shown in Figure 1.

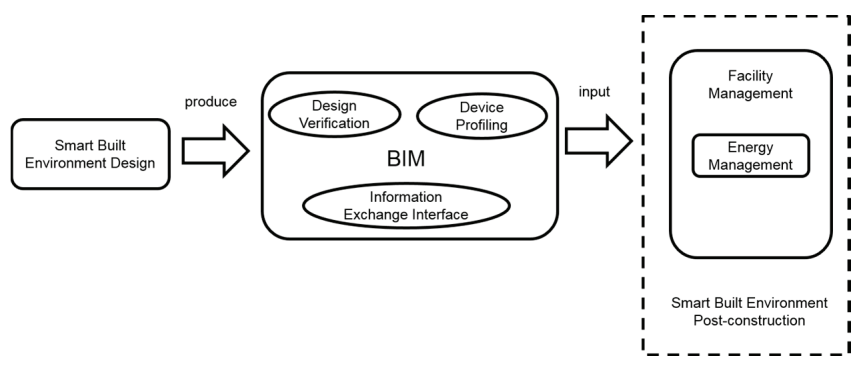

Figure 1. Role of building information modelling (BIM) in smart built environments.

\section{Designing Smart Built Environment with BIM}

As discussed in the previous section, BIM has the potential to support the entire life-cycle of the SBE. Therefore, we started by investigating how existing BIM platforms can be further developed to better support the design of SBE. To date, the most popular BIM software is the Revit suite from Autodesk and Archicad from Graphisoft. In this work, we have conducted our SBE design in BIM with Revit, but similar work can be conducted as well in Archicad.

\subsection{Embedding Smart Objects in BIM}

The intention is to profile smart objects with Revit during the SBE design phase. The produced BIM model with smart objects should be correctly exported as an International Foundation Class (IFC) file, so that other BIM tools can parse the information. We adopted three methods for the profiling: IFC shared parameter, family property parameter and the mark tag. The work in [20] 
demonstrated how sensors can be modelled in Revit, and we took a similar approach, which uses the IFC shared parameter field to indicate the sensor type in IFC, as shown in Figure 2a. For actuators, new properties can be added to the building element that is being controlled by the actuator. Therefore, new BIM artefacts, such as a smart window (e.g., capable of self-actuating to adjust its light transmission properties), can be created using the original window family with added actuator properties.

The IFC shared parameter enables the new smart object family to be a compatible type in BIM, i.e., exportable and parsable in IFC format. However, to fully model an SBE design, we have to provide additional information. For that purpose, we utilize the family property parameters for a type of smart object and mark tag for an individual smart object.

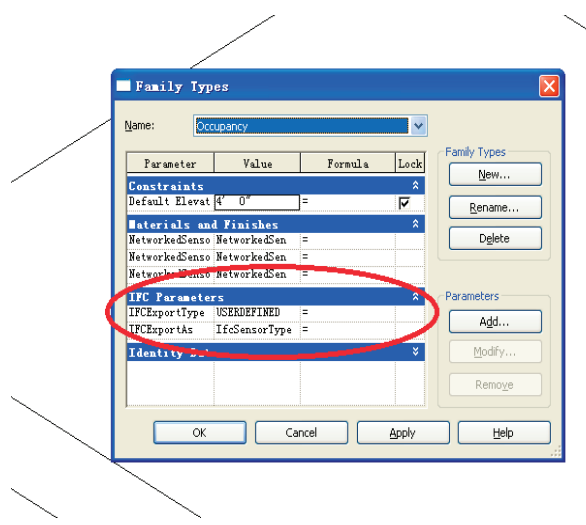

(a)

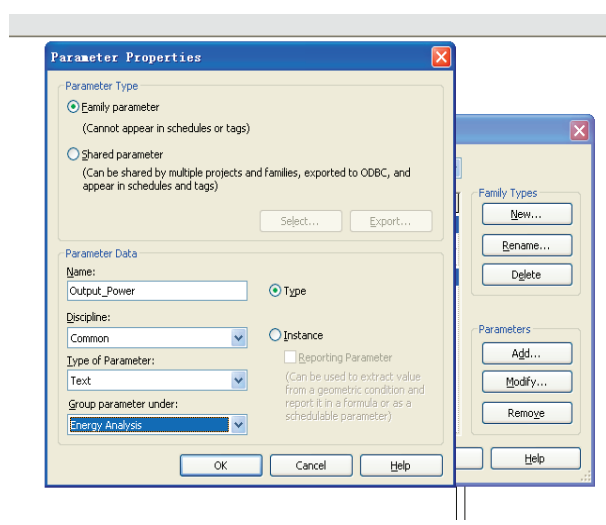

(b)

Figure 2. (a) Extending BIM design using the "IFC shared parameter"; (b) Adding new parameter properties in Revit.

\subsubsection{Spatial Interaction Information}

One of the most important factors to consider when designing SBE is the spatial interactions between a smart object and its physical surroundings. For sensors, it has been referred to as the range space or the effective sensing space area [12], which is the foremost element to consider when planning their placement layout during the design of SBE. Different sensors types may have different range space characteristics. For an infrared motion sensor, its range space may have a shape like a sector of a circle. For a temperature sensor, its range space may be spherical-like. For simplicity, we represent the range space of a sensor by a range radius and range angle, and its axis is determined by the surface to which it attaches, as shown in Figure 3. 


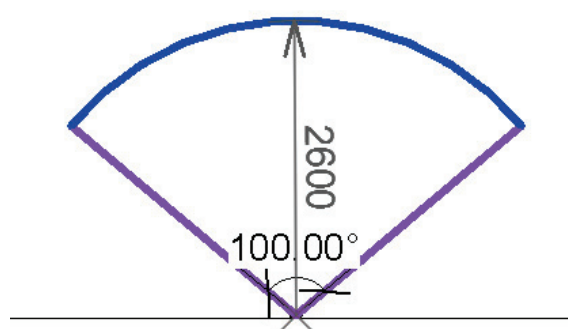

(a)

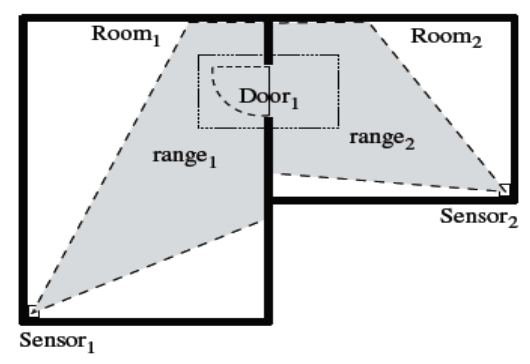

(b)

Figure 3. (a) Example of the range space of a sensor with range radius of $2.6 \mathrm{~m}$ and a range angle of $100^{\circ}$; (b) the door's operational space should be within the range space of the two monitoring sensors.

\subsubsection{Informational Exchange Interface}

For the information exchange interface, which describes the data input/output behaviour of the smart object, family property parameters are added to the smart object family. Since this work focuses on energy management, we specify parameters in the energy analysis properties.

As illustrated in Figure 2b, in order to enable the BIM file (in IFC format) to provide an information exchange interface for the facility software, family property parameters are designed to be a mapping from IFC text to the device software programming interface, which can be a middleware or another BIM software. Three types of smart object data operations are defined:

- Output: the interface from which external software can read the output (generated power, grid signal, or sensed data) of the device. The format in the IFC file is: "Output_xxxx".

- Input: the interface from which external software can read the power generation or consumption status of the device. The format in the IFC file is: "Input_xxxxx".

- Control: the interface from which external software can control the operation of the device, the tilting angle of the PV panel/wind turbine, the power consumption of smart appliances compatible with BACnet (Building Automation \& Control networks) standard, etc. The format in the IFC file is: "Control_xxxxx".

\subsubsection{Identification and Service Mapping}

Finally, to map a smart object from BIM to an individual real-world device, the mark tag in BIM is used. The BIM software, which parses the produced BIM file, reads the mark tag for the smart object and determines additional runtime information, e.g., the Internet Protocol (IP) address, of the device from a database. This tag is also used to map a smart object to a service descriptor file, which will be detailed in the next section.

Using Revit, we created an SBE design, which is modelled as a smart house with sensors, actuators, smart meters, PV panel and wind turbine. After completing the BIM design, a BIM file in IFC format is generated, as shown in Figure 4. 


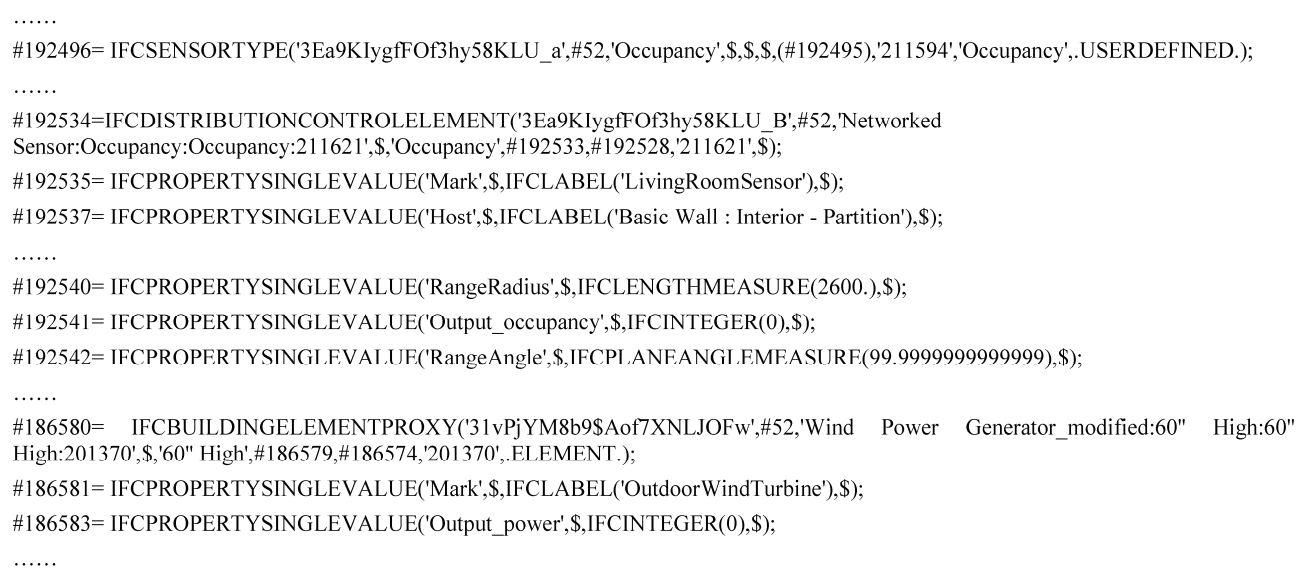

Figure 4. Code segment of the BIM IFC file.

\subsection{Design Verification Framework for SBE}

Recall that SBE serves to enhance the building's efficiency, security and comfort of its human occupants by being aware of the state of the environment and performing autonomous intelligent actions. Such actions performed by the SBE can be viewed as provisioning a type of service to the building owner or occupants. In literature, these services are also referred to as real-world services (RWS) [7]. Designing a set of adaptive RWS with various smart objects could become an integral part of future SBE design.

During the design phase of SBE, uncertainties may arise that could affect the service integrity and performance. For example, there could be doubts about whether the sensors are placed in the best locations to execute their tasks or whether there are conflicting objectives/requirements between different services. If such uncertainties can be verified during the design phase of the SBE, it may reduce the amount of rework necessary after the building is built. Furthermore, it can be useful for as-built verification, e.g., when new smart objects and services are to be introduced to an already constructed SBE. With this motivation, we propose an SBE design verification framework with BIM, as outlined in Figure 5.

In this framework, a three-layer verification is adopted to verify the SBE design. The three layers are: smart object, single service and inter-service performance verification. The input to the framework is the BIM file produced as discussed in Section 4.1 (in IFC format), which contains the design information of the building and associated smart objects. The performance verification criteria are modelled using XML, which are detailed in the following sections. 


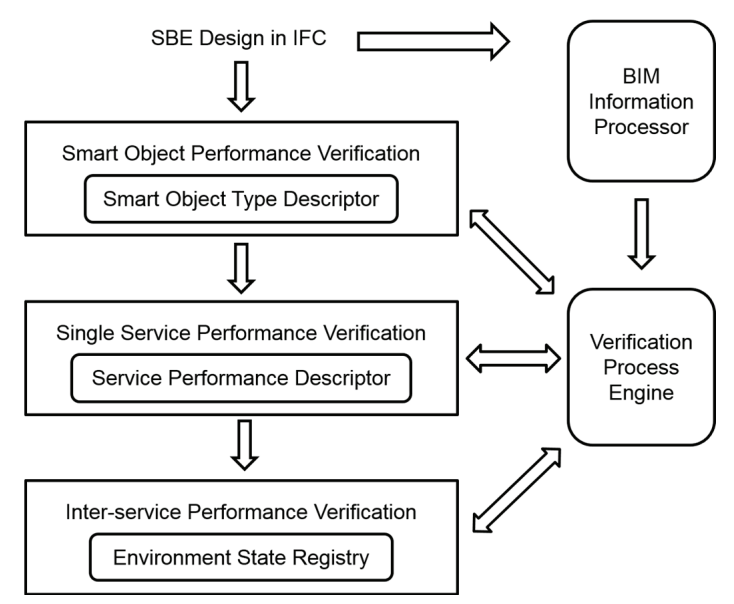

Figure 5. Design verification framework for smart built environments (SBEs) with BIM.

\subsubsection{Smart Object Performance}

The performance of a smart object can be impacted to different degrees by its surrounding building elements, depending on the type of smart object. For example, a temperature sensor may be mistakenly placed next to a furnace or air conditioner, which can interfere with its proper operation. In [22], it was shown that the performance of an optical sensor can be greatly influenced by the material properties of the target surface that it is sensing. The actual verification criteria used are specific to the type of smart objects. A smart object type descriptor (STD) is created as an external XML file to describe the performance constraining factors of each smart object. An example STD is shown in Figure 6a. The verification engine then parses the description and analyses the spatial interactions between the smart object and its surrounding building elements.

\subsubsection{Single Service Performance}

An RWS is defined on a set of smart objects and building elements involved in delivering the service to the building occupants/owner/manager. For example, as shown in Figure $3 b$, a room entry monitoring service is defined on an infrared motion sensor in the room and the door that is within its range space.

Likewise, a service performance descriptor (SPD) is created as an XML file to describe the smart objects and building elements that constitute each service. An example SPD is shown in Figure 6 b. Each RWS could be constituted by one or more smart objects. When verifying a service, the verification engine parses the SPD and analyses the smart object's performance constraining factors (from STD) and building element properties, such as operational space and material information (from the IFC file) for their impact on the service performance. 


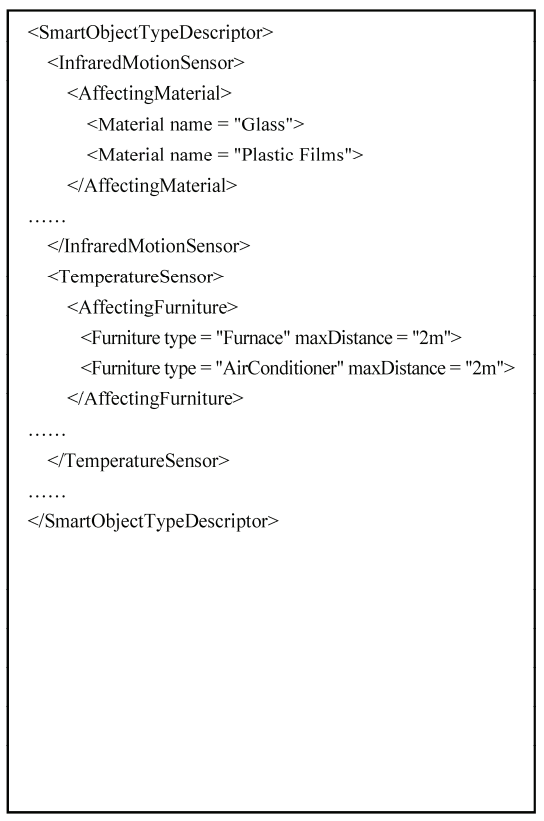

(a)

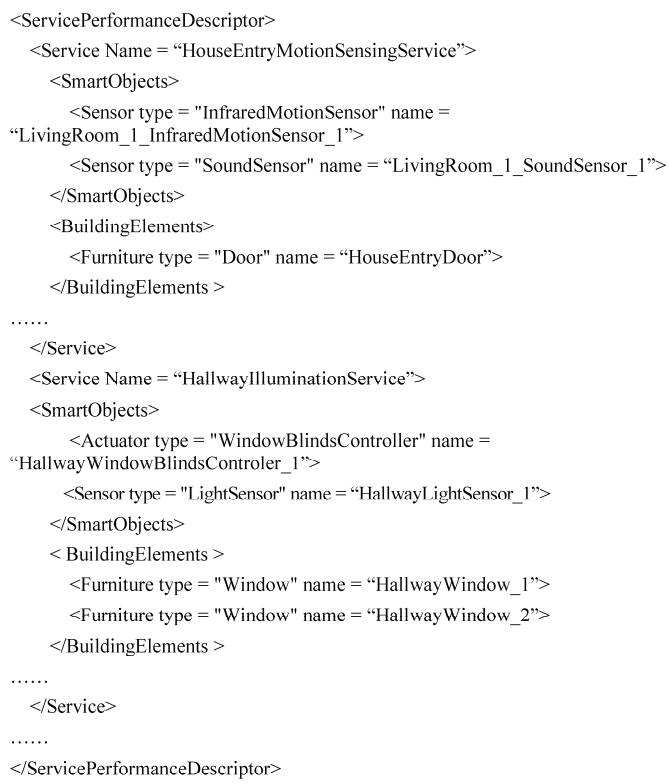

(b)

Figure 6. (a) Example of a smart object type descriptor; (b) Example of a service performance descriptor.

\subsubsection{Inter-Service Performance}

In SBE, each RWS works autonomously in most cases. However, an event may occur that triggers several services, either because the event simultaneously satisfies the trigger conditions of multiple services or the result of one service leads to another, i.e., chain of services [9]. The confluence between services can be complicated and may not be completely anticipated by the designer. Some consequent impacts can be collaborative and beneficial to the service users, while some can be conflicting and degrade the service quality as a whole.

For example, during a hot summer, an occupancy sensor senses a user's presence and instructs the HVAC system to cool the room, while a motion sensor in the room recognizes the same user to be reading a book and instructs the window to roll up its shades to improve illumination. However, the incoming sunshine heats up the room and causes the former cooling service to take more time and consume more energy to achieve its targeted temperature.

Such service conflicts undermine the quality of an SBE design and impact the performance of the conflicted services. In the literature, this conflict of services is sometimes referred to as feature interaction [9], which could be spotted during the design phase by identifying their triggering events and resulting environment state change.

To verify an SBE design against service conflicts, each service registers its triggering/affecting environment factors, which are also profiled in the SPD, with the environment state registry (ESR). The verification engine performs environment state reasoning by analysing the location of smart 
objects that constitute the different services and the environment factors that they affect. Using the previous example of service conflict, the occupancy sensor and motion sensor may or may not have overlapping range spaces, but they share a common room space. Furthermore, if the room space of two smart objects with common environment factors can be connected through opening a door, a warning should be generated for the design. Once again, BIM provides data on the room space where a smart object resides, and the verification engine queries the ESR and checks against the other services defined on smart objects in the same or adjacent room connected by a door, as shown in Figure 7.

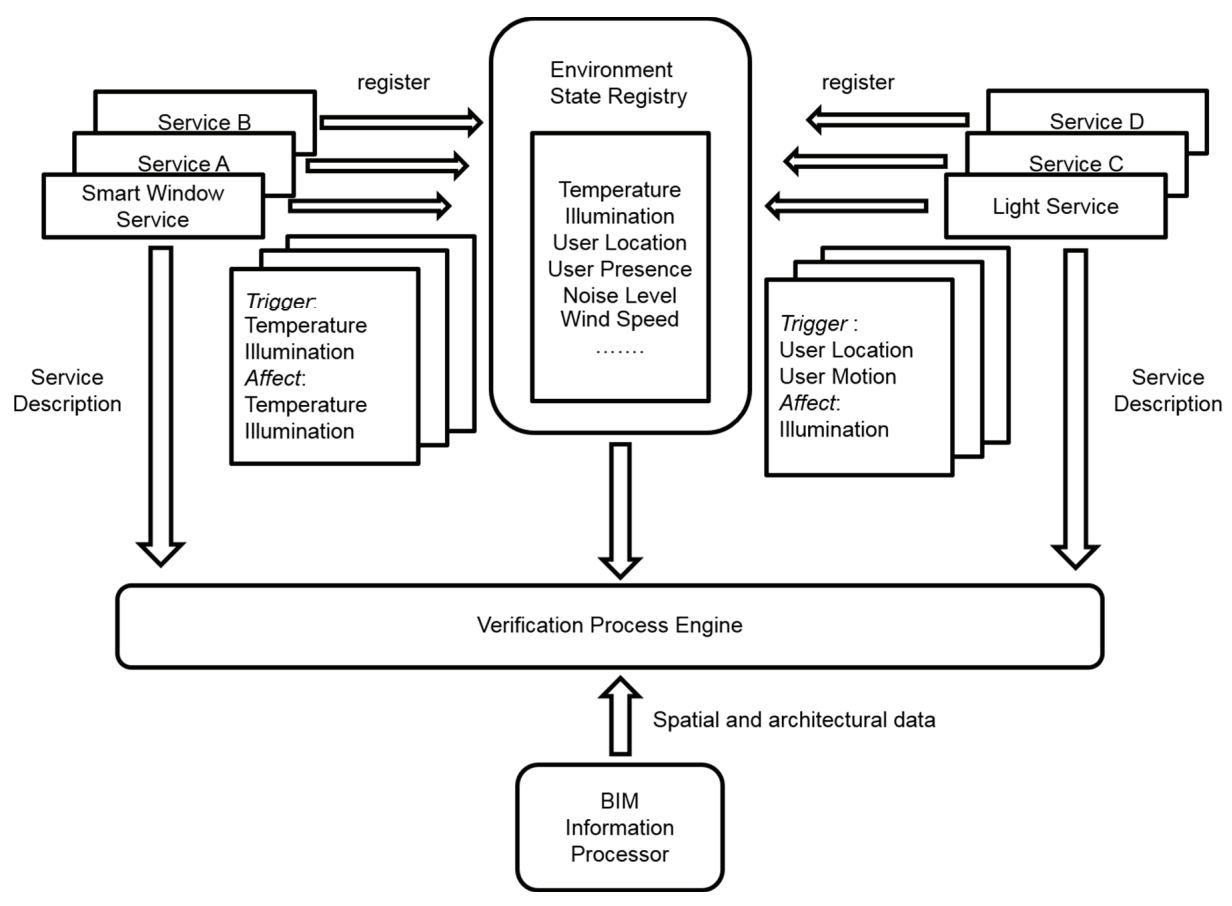

Figure 7. Verification with environment state registry.

\section{Integrating BIM in Post-Construction Facility Management}

The BIM file generated from Revit and validated using the verification framework in the previous section is an output from the SBE designer or architect during the building design phase. In the post-construction facility management phase, the building manager can apply the information in this BIM file to perform day-to-day building management, including energy management.

To parse the BIM file and read the profiled information, there are two possible options for the design of this research. The first is to extend Revit using a native software development kit (SDK) to perform the energy management task. The second is to develop a standalone BIM tool as a separate energy management engine. After much contemplation, we decided to go with the second option, as we believe that building managers will be more familiar with using a building management system 
(BMS) for their everyday work than with Revit, which is intended as a computer-aided design (CAD) tool for building designers and architects.

Therefore, we opted for the second option, and a stand-alone BIM tool for energy management has been developed. We developed the tool using the extensible building information modelling [23] toolkit, which provides IFC parsing and 3D presentation utilities.

As different smart objects may have different data input/output interfaces, the properties parameter specified in our BIM design in the previous section provides a convenient way for the BIM tool to handle such low-level operations. An adapter layer is designed in this research to process the requests from BIM software, which parses the IFC file and demands data exchange for the smart object. The mapping/parsing operation is illustrated in Figure 8. The software architecture for our developed BIM tool is shown in Figure 9.

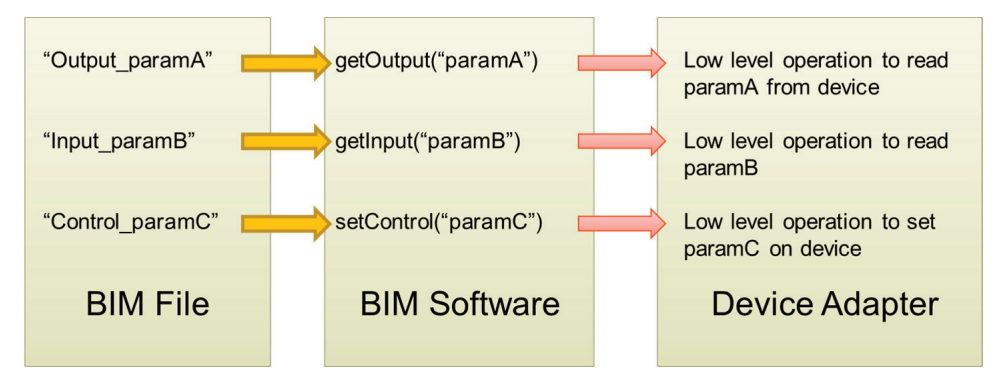

Figure 8. Mapping from the BIM file to the software information interface programming.

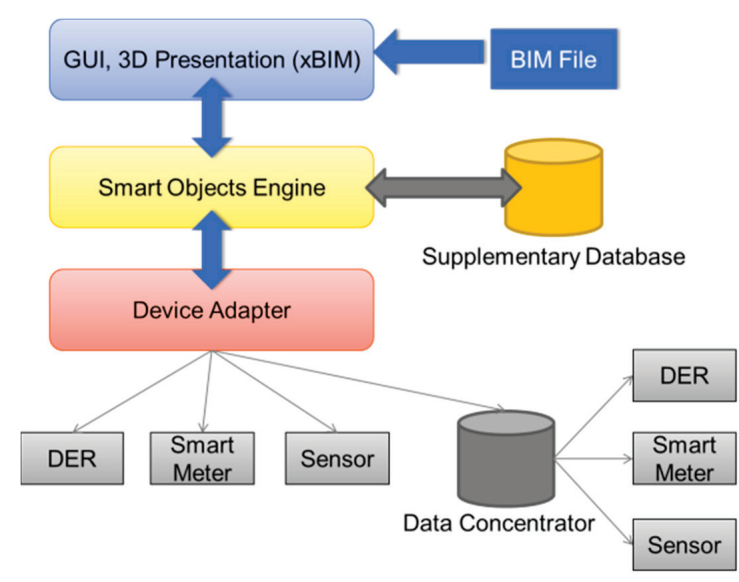

Figure 9. BIM-based energy management platform software architecture. DER, distributed energy resource.

Real-time data from the DERs, smart meters and sensors are collected and stored in a database. Figure 10 shows an instance of the real-time monitoring data from a living room sensor displayed to the user, i.e., home owner or building manager. 
With real-time data from the smart objects, energy management and analysis in BIM software are facilitated and achieved. Real-time generation data of on-site DERs show the current energy production capacity and indicate how many loads can be supplied off the power grid. Weather, temperature, building and occupant data from sensors form a view of the present and future energy generation/consumption, as shown in Figure 11. The pricing information from the smart meter allows the building to perform demand response actions in coordination with the power grid.

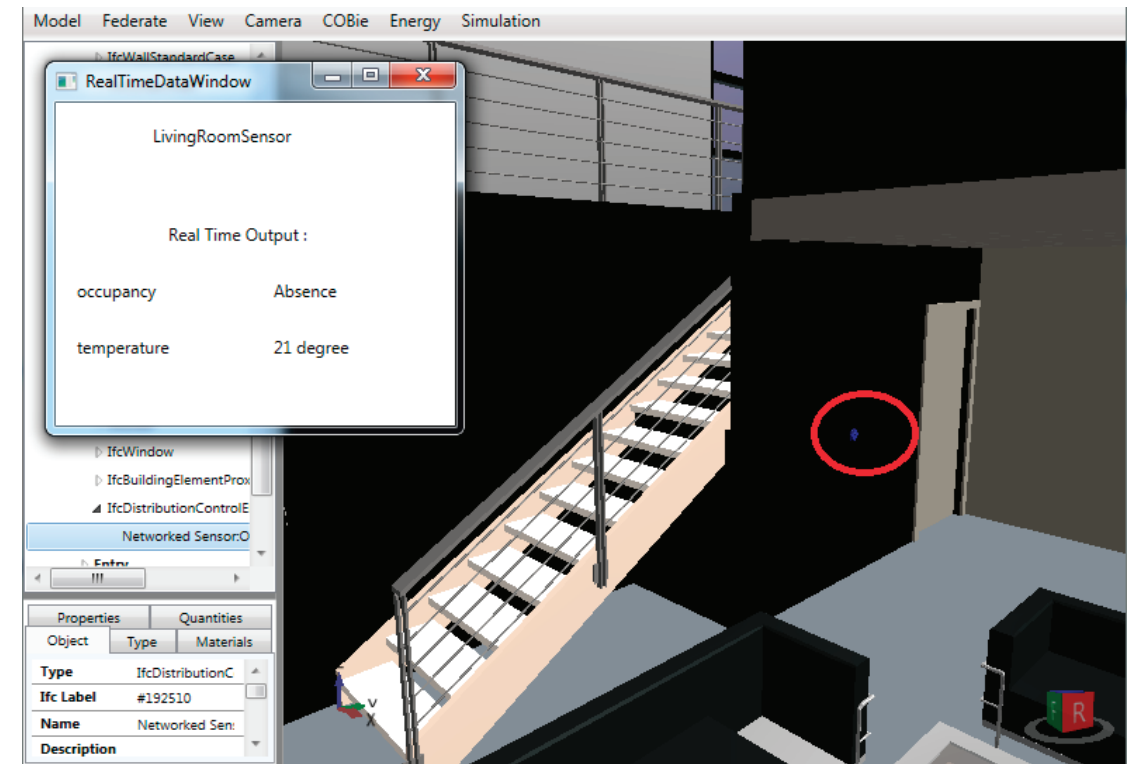

Figure 10. Real-time monitoring data display to the user.

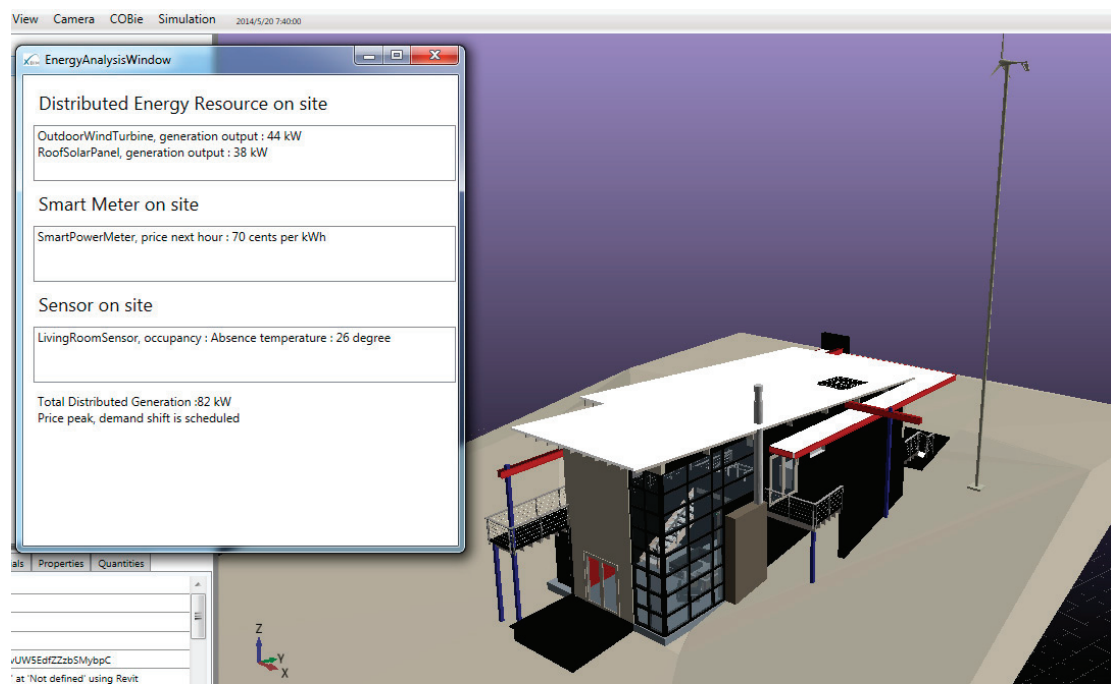

Figure 11. Energy analysis functionality in smart built environment. 
In our BIM tool, we demonstrated the energy management functionality through a simulation of demand shifting. When the real-time pricing information from the smart meter reaches a user-defined threshold, the software triggers the demand shift process and transmits control commands to the energy-consuming appliances.

As future work, we are working towards an advanced BIM-based building management platform for smart buildings to host smart grid services and applications, such as virtual power plant and demand response, as shown in Figure 12.

\section{Smart Grid Interface}

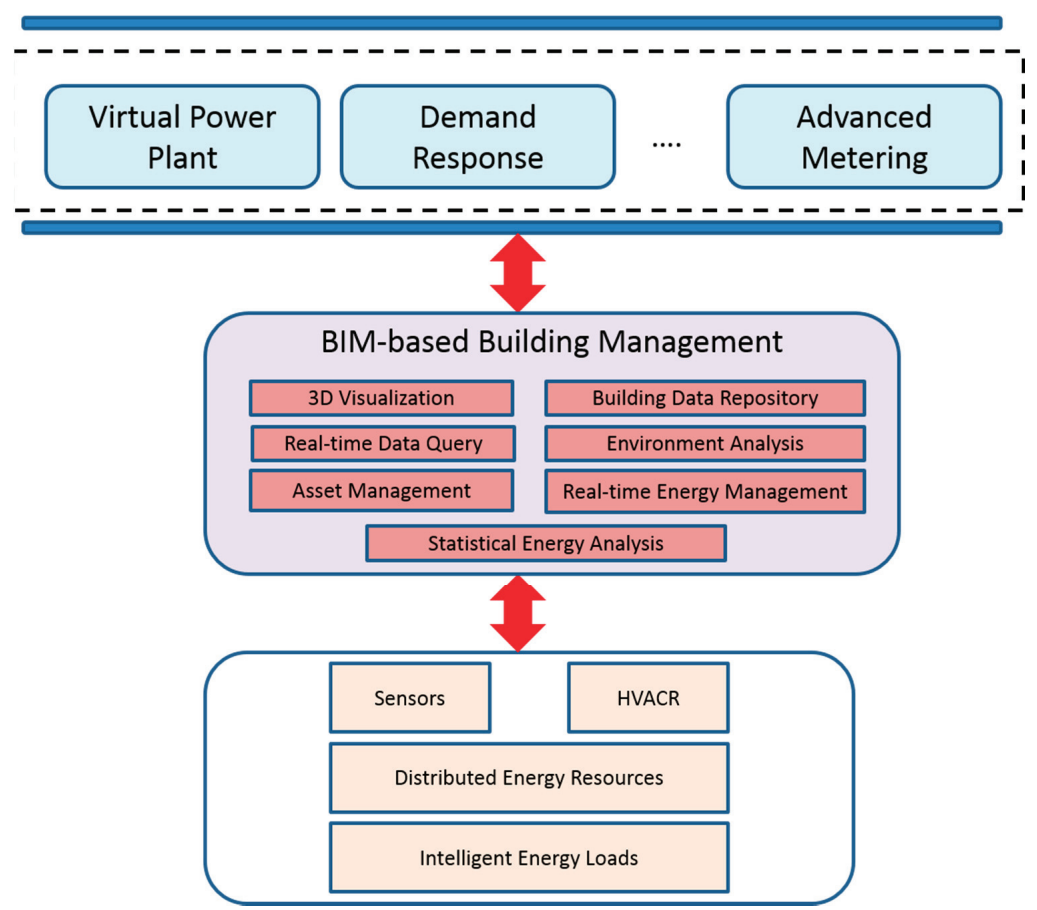

Figure 12. Proposed BIM-based building management for a smart grid.

\section{Conclusions}

In this paper, the findings and experiences gained from an investigation into how BIM can be developed and utilized for smart built environments are reported. Our work covers the investigation of BIM-based methodologies for pre-construction design and verification and post-construction facility management of smart built environments in the smart grid era. A basic, but functional, prototype of a smart house energy management system using Revit and the xBIM toolkit was also implemented and successfully demonstrated. We are currently extending our work to develop BIM software tools for automated management of BACnet-compliant HVACR (Heating, Ventilating, Air Conditioning and Refrigeration) systems and investigating the information fusion and decision system of smart grid applications for future smart-powered buildings. 


\section{Acknowledgments}

This work has been supported in part by a research assistantship grant from Auckland University of Technology for Jianchao Zhang. The authors would also like to thank Andrew Douglas for facilitating the access to the software resources used in this research.

\section{Author Contributions}

The topic and scope of this research were originally defined in a research proposal by Boon-Chong Seet and Tek Tjing Lie on BIM for smart-powered buildings. Jianchao Zhang conducted the research and implemented the proposed solutions under the supervision of Boon-Chong Seet and Tek Tjing Lie, who contributed their expertise on smart environments and smart grids, respectively. The paper was drafted by Jianchao Zhang and revised by Boon-Chong Seet.

\section{Conflicts of Interest}

The authors declare no conflict of interest.

\section{References}

1. Sabol, L. Building Information Modelling and Facility Management. In Proceedings of the IFMA World Workplace, Dallas, TX, USA, 15-17 October 2008.

2. Teicholz, P. BIM for Facility Managers; John Wiley \& Sons: Hoboken, NJ, USA, 2013; p. 332.

3. Talebi, S. Rethinking the Project Development Process through Use of BIM. In Proceedings of the 2nd BIM International Conference on Challenges to Overcome, Lisbon, Portugal, 9-10 October 2014.

4. Nakashima, H.; Aghajan, H.K.; Augusto, J.C. Handbook of Ambient Intelligence and Smart Environments; Springer: New York, NY, USA, 2010; p. 1293.

5. International Energy Agency. Sustainable Buildings. Available online: http://www.iea.org/topics/energyefficiency/subtopics/sustainablebuildings/ (accessed on 30 November 2014).

6. Van der Meer, S.; Jennings, B.; Barrett, K.; Carroll, R. Design Principles for Smart Space Management. In Proceedings of the 1st International Workshop on Managing Ubiquitous Communications and Services (MUCS), M-Zones Whitepaper, Waterford, Ireland, 11 December 2003.

7. Umakoshi, K.; Kambayashi, T.; Yoshida, M.; Takemoto, M.; Matsuo, M. S^3: Smart Shadow System for Real World Service and Its Evaluation with Users. In Proceedings of the IEEE/IPSJ 11th International Symposium on Applications and the Internet (SAINT), Munich, Germany, 18-21 July 2011.

8. Dong, J.S.; Feng, Y.; Sun, J. Sensor Based Designs for Smart Space; Technical Report; Computer Science Department, National University of Singapore: Singapore, 2004.

9. Inada, T.; Igaki, H.; Ikegami, K. Detecting service chains and feature interactions in sensor-driven home network services. Sensors 2012, 12, 8447-8464. 
10. Guinard, A.; McGibney, A.; Pesch, D. A Wireless Sensor Network Design Tool to Support Building Energy Management. In Proceedings of the First ACM Workshop on Embedded Sensing Systems for Energy-Efficiency in Buildings, Berkeley, CA, USA, 4-6 November 2009.

11. Jeng, T. Toward a ubiquitous smart space design framework. J. Inf. Sci. Eng. 2009, 25, 675-686.

12. Bhatt, M.; Dylla, F.; Hois, J. Spatio-Terminological Inference for the Design of Ambient Environments. In Spatial Information Theory; Springer: Berlin, Germany, 2009; pp. 371-391.

13. Becerik-Gerber, B.; Jazizadeh, F.; Li, N.; Calis, G. Application areas and data requirements for BIM-enabled facilities management. J. Constr. Eng. Manag. 2011, 138, 431-442.

14. Attar, R.; Hailemariam, E.; Glueck, M.; Tessier, A.; McCrae, J.; Khan, A. BIM-Based Building Performance Monitor. In Proceedings of the SimAUD Conference, Symposium on Simulation for Architecture and Urban Design, Orlando, FL, USA, 12-15 April 2010.

15. Onuma. Onuma System. Available online: http://onuma.com/products/OnumaPlanningSystem.php (accessed on 30 November 2014).

16. Lavelle, M.R.; Onuma, K. Virtual Real Time Information System: Moving to Cloud-Based Building Management. Available online: http://www.lavelleenergy.com/index.php/ component/content/article/all-buildings/86-virtual-realtime-information-system (accessed on 30 November 2014).

17. Arnold, G.W. Challenges and opportunities in smart grid: A position article. Proc. IEEE 2011, 99, 922-927.

18. East, B. Construction-Operations Building Information Exchange (COBie). Available online: http://www.wbdg.org/resources/cobie.php (accessed on 30 November 2014).

19. Park, S.O.; Park, J.H.; Jeong, Y.S. An efficient dynamic integration middleware for cyber-physical systems in mobile environments. Mob. Netw. Appl. 2013, 18, 110-115.

20. Autodesk. Placing Sensors in the Revit Model. Available online: http://www.digital210king.org/blog.php?p=30 (accessed 30 November 2014).

21. Resendes, S.; Carreira, P.; Santos, A.C. Conflict detection and resolution in home and building automation systems: A literature review. J. Ambient Intell. Humaniz. Comput. 2014, 5, 699-715.

22. Marza, A.O.; Avram, I.M.; Tamovan, I.G. An Analysis of Target Materials Influence on Optical Sensors Performance. In Proceedings of the IEEE International Conference and Exposition on Electrical and Power Engineering (EPE), 25-27 October 2012; pp. 85-87.

23. The xBIM Toolkit. Available online: http://xbim.codeplex.com/ (accessed on 30 November 2014). 


\title{
Driving Innovative Thinking in the New Zealand Construction Industry
}

\section{Mohammadali Noktehdan, Mehdi Shahbazpour and Suzanne Wilkinson}

\begin{abstract}
This paper examines the relationship between innovation and productivity improvement in the construction industry. It is argued that this relationship is not well understood due to lack of in-depth understanding of innovation in construction. To overcome this obstacle, the authors present a multi-dimensional innovation classification system which aims at better defining and classifying what is meant by innovation in construction. The use of this classification system is demonstrated by applying it to a database of 500 innovations reported by the construction alliance, the Stronger Christchurch Infrastructure Rebuild Team. The results clearly demonstrate the diversity of types, degree of novelty and performance improvement benefits among construction innovations. Such diversity means that the impact of the reported innovations on productivity and performance are of different levels of significance. The classification system developed in this study can be used by construction organisations and alliances in the future to develop more detailed methods of calculating innovation performance indicators, based on the innovation type, novelty and benefits factors. By using this system, they can also put in place mechanisms to influence the types of innovation developed in their projects with the aim of maximising their productivity performance.
\end{abstract}

Reprinted from Buildings. Cite as: Noktehdan, M.; Shahbazpour, M.; Wilkinson, S. Driving Innovative Thinking in the New Zealand Construction Industry. Buildings 2015, 5, 297-309.

\section{Introduction}

The Construction industry is critical to the functioning of a domestic economy in many countries [1]. In New Zealand, the construction industry is one of the largest sectors of the economy accounting for $8 \%$ of total employment in the country [2]. However, in spite of its importance to the national economy in terms of size, the construction industry seems to be lagging other sectors in terms of productivity [3]. In 2010, the construction industry in New Zealand established the Building and Construction Sector Productivity Partnership to actively address the issue of low productivity in the sector. Although the early focus was on identifying and quantifying the problems that lead to low productivity, over the recent years the focus has shifted to problem solving and addressing the cultural and mechanistic change that is needed to resolve the well documented issue [3].

One of the areas that the partnership has identified as critical for achieving significant improvements in the sector's productivity is innovation. The ultimate goal of $20 \%$ productivity improvement by 2020 cannot be achieved by repeating the old ways of doing things. New innovative approaches are required in order to significantly improve performance at the same cost or maintain the same level of performance at much lower cost. Unfortunately the construction industry is one of the least innovative sectors compared to other industries such as manufacturing and traditional services [4]. The research and development report produced by Statistics New Zealand 
indicates that $\mathrm{R} \& \mathrm{D}$ expenditure in the construction industry accounts for a low $5 \%$ of the total expenditure in the sector [5]. Indeed, this problem is not limited to New Zealand as internationally the construction industry is seen as a traditional or low-technology sector with low levels of expenditure on activities associated with innovation [6].

Furthermore, it appears that the link between innovation and productivity in construction industry is not well understood. Loosemore [7] observes that in spite of many recent developments in the field of construction innovation, none of these studies feature in the mainstream construction productivity literature.

In contrast, the link between productivity and innovation has been well researched and explored in the manufacturing literature [8]. However, while certain parallels can be drawn from the manufacturing literature, it is generally agreed that there are significant differences between manufacturing and construction. Koskela and Vrijhoef [9] identified the one-of-a-kind nature of construction project, site production, temporary multi organization and regulatory intervention, as differentiating characteristics of the construction industry accounting for lower innovation. Reichstein et al. [4] also identified a number of "liabilities" which sets construction industry apart from other innovative industries such as manufacturing. These are Immobility, Project-based, Uncertainty of demand, Smallness, Separation and Assembly.

There is a need for more construction specific research to explore the relationship between innovation and productivity. The authors contend that while productivity is a well-defined term, innovation in contrast is poorly defined for the construction industry and is only understood in abstract terms. This is a major obstacle in the attempt to clarify the relationship between innovation and productivity in the construction industry. The study presented in this paper, aims to make a contribution in this area by demonstrating the diversity of various types of innovation present in a construction project and the varying degrees of impact these innovations have on productivity indicators.

The paper will first present this classification system from a theoretical perspective and illustrates how it can be used to better understand the relationship between innovation and productivity. The use of this classification system is then demonstrated by applying it to a database of 500 innovations reported by the construction alliance, the Stronger Christchurch Infrastructure Rebuild Team (SCIRT), responsible for rebuilding the horizontal infrastructure after the earthquakes in Christchurch. SCIRT is an organisation who put innovation as one of the main key performance indictors (KPIs) for its construction project delivery team. The results will highlight the diversity of innovation types that have been reported by the alliance group, and the classification system used in the paper makes sense of these innovations, and suggests ways of improving productivity through innovation use in construction.

\section{Defining Innovation}

Productivity is generally defined as a ratio between output produced and input used [10]. For instance, labour productivity can be calculated as a ratio of hours of labour worked (input) and units produced (output). More holistic definitions of productivity include a number of performance dimensions for units of input and output, such as quality, cost, time and safety. Innovation on the other hand is more ambiguous in its definition. Brown [11] provides a broad definition of 
innovation as "doing things differently or better across products, processes or procedures for added value and/or performance". West and Altink [12] provide a similar, but perhaps more complete, definition of innovation as the "intentional introduction and application within a role, group or organisation of ideas, processes, products or procedures, new to the relevant unit of adoption, designed to significantly benefit the individual, the group, the organisation or the wider society".

When comparing the above definitions and other popular definitions of innovation in the literature, three key defining elements of innovation can be identified. First is the type of innovative idea or invention. The Oslo Manual on collecting and interpreting innovation data [13] outlines four types of innovations: product, process, marketing and organisational. Second is novelty. The minimum entry level for an innovation is that it must be novel or "new to the firm" [13]. Degree of novelty or "newness" can be defined as the extent of uncertainty associated with the implementation of the innovative idea within the context of its application [14]. Slaughter [15] divided the Novelty of the construction innovation in five different levels of Incremental, Modular, Architectural, System and Critical. The third is benefit or improvements in performance. In construction, traditionally performance has been measured in terms of cost, time, quality and safety [16]. Recently, environmental measures have also become an important performance indicator in construction projects [17].

Each of these elements (type, novelty, and benefit) can alter the significance and type of the impact that a given innovation has on productivity. In terms of type, whether the innovative ideas are a product, process or an organisational method results in different type of impact on productivity. For instance, process and organisational innovations are mainly concerned with the construction phase and thus will have a direct impact on the project's productivity performance. On the other hand, product innovation may have much less significant impact on the production phase but much larger impact once the building or infrastructure is in operation. In terms of novelty, the degree of novelty determines the significance of the impact the given innovation may have on productivity. For instance, an incremental change results in much less significant impact on performance than a more radical and disruptive change. Finally, different innovations would improve different set of performance indicators, thus having very different impacts on productivity.

It is therefore proposed that in order to better determine the impact and nature of the relationship between given innovations and overall project productivity, types of innovation, novelty and the specific performance indicators being improved must first be determined. This requires an innovation classification system, which can be used to categorise the innovations implemented in a project.

\section{Innovation Classification System}

A multi-dimensional classification system is presented, based on review of relevant construction and innovation management literature regarding the elements discussed previously. After a review of the literature, it was found that the most commonly accepted innovation classifications have mainly been developed by analysing innovation within the manufacturing and services context. As mentioned previously, there are significant differences between construction and other industries. 
Consequently, in order to develop an appropriate innovation classification system for the construction industry, modifications were made to these classification systems and where appropriate new categories were developed. This section will briefly outline each dimension and provide a definition for each classification category.

Innovation Type: The authors found it necessary to distinguish between development or utilisation of innovative construction materials or componentry and the development of innovative designs and features for buildings or infrastructures. Therefore, the product innovation category was limited to cover new materials or products used in the construction phase, and a new category was added, called Design, to account for the innovative design features introduced at the design phase of the project. Furthermore, guided by the construction technology classification system developed by Tatum [18], the process innovation category was divided into two sub-categories of Tools and Function.

- Product: Product Innovation involves all new construction materials and products developed in the project or introduced to the project and used within the construction process.

- Design: Design Innovation is related to new and innovative plans, designs, sketches or concepts for the final building or infrastructure that is being developed in the project.

- Tool: The Tool Innovation involves the development or implementation of novel construction machinery equipment or tools in the construction project.

- Function: The Functional Innovation refers to new tasks developed or introduced in the construction project and associated management processes.

After further consultation with industry experts two other categories were added where a combination of the previous sub-categories could exist:

- Technology (Design + Product): The New Technology refers to the new design that is coupled with a new material or product.

- Method (Tool + Function): The method innovation is the combination of the Tool and Function Innovation that involve both a new tool or equipment and new tasks that are usually related to the new tool.

Innovation Novelty: Typically the innovation literature distinguishes between incremental and radical innovations. However, Slaughter [15] provides a more detailed categorisation of novelty within construction innovation context. These categories are Incremental, Modular, Architectural, System and Critical.

- Incremental: Incremental Innovation is a small change, based upon current knowledge and experience. It is often the result of continuous improvement initiatives and on-the-job problem solving.

- Modular: Modular Innovation entails a significant level of novelty in one area of a system, but without impacting the other components of the system. Modular innovations may be developed within an organization and implemented without much negotiation with parties involved in the development or selection of other components.

- Architectural: Architectural Innovation involves a small change within a component of a system, which results in major changes in the links to other components and systems. 
The distinction between modular and architectural innovations is made on the region of the change and, specifically, the degree of interaction with other components of the system.

- System: System Innovations are identified through their integration of multiple independent innovations that must work together to perform new functions or improve the facility performance as a whole.

- Critical: Critical Innovation is a breakthrough in science or technology that often changes the character and nature of an industry. While incremental innovations occur constantly, critical innovations are rare and unpredictable in their appearance and in their impacts.

Innovation Benefit: This dimension of the classification system deals with the type of performance improvement that is achieved through implementation of the new innovative ideas. As mentioned previously, the following performance indicators will be used to distinguish between the types of benefits that are delivered by the given innovations.

- Cost: Direct cost savings or better utilisation of resources.

- Time: Reduction in lead-times or increasing speed for the project or sub-tasks.

- Quality: Improvements in degree of conformance with specifications and/or satisfaction of stakeholders with the outputs of the construction project.

- Safety: Improving safety, health and wellbeing of the employees and public during and after the construction project.

- Environment: Reducing adverse impact of the construction processes as well as the final building or infrastructure on the natural environment.

- Community: Reducing adverse impact on communities affected by the construction project and improving communication with the stakeholders.

\section{Case Study}

SCIRT is an organisation established under an alliance agreement and is responsible for rebuilding horizontal infrastructure in Christchurch following the earthquakes of 2010 and 2011. Innovation was given a special consideration from the outset, when the SCIRT alliance was formed. In fact, members of the alliance were encouraged to innovate and report on their innovations on a monthly basis as one of their KPIs. These KPIs were linked directly to the pay/reward aspect of the contract. As a result, the alliance members had ample motivation to report their innovations. To date more than 500 innovations have been reported by SCIRT. This has provided a unique opportunity to analyse and better understand the relationship between construction innovation and productivity improvements. The authors were given full access to SCIRT's innovation database. For each reported innovation, the database contained a unique identification number, description of the innovative idea, its potential benefits and information regarding which member organisation had initiated the innovation. Some of the reported innovations were also accompanied by pictures or sketches to better describe the innovation. Each of the reported innovations were analysed and categorised based on the innovation classification system outlined in the previous section. The categorisation process was carried out mainly by one researcher to ensure consistency of interpretation across the data set. Once completed, 
20 innovations were selected randomly and categorised by two other researchers using the classification system. This was done to make sure the classification system was being applied appropriately. Any uncertainty around the definition was clarified through this process.

\section{Results}

In order to better understand the spread of various categorisations of innovations developed by SCIRT, the data was analysed using pivot-table functionality of MS Excel. A pairwise analysis was also conducted to identify any potential trends which may lead to insights about the nature of innovation activity at SCIRT. The following section presents the summary of the results from this analysis.

\subsection{Spread of Innovation}

Figures 1-3 represent the spread of innovation categories in each dimension of the classification system: type, novelty and benefit. Most innovations in the SCIRT database seem to be made up of tools or functions in terms of innovation type, and modular or architectural in terms of novelty. They also appear to deliver a wide spread of performance benefits, mainly dominated by quality, time and cost.

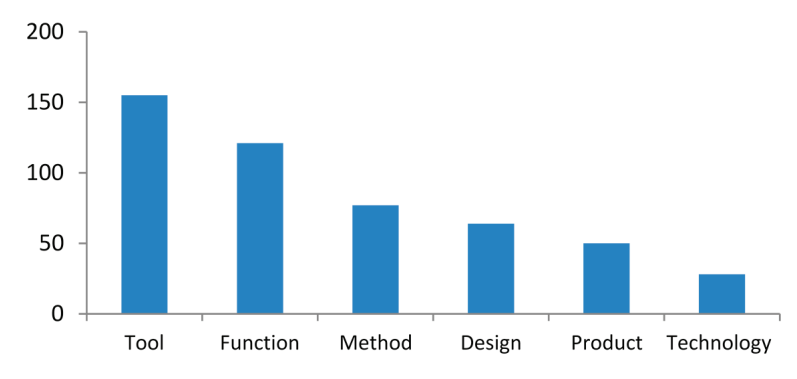

Figure 1. Innovation classification based on Type.

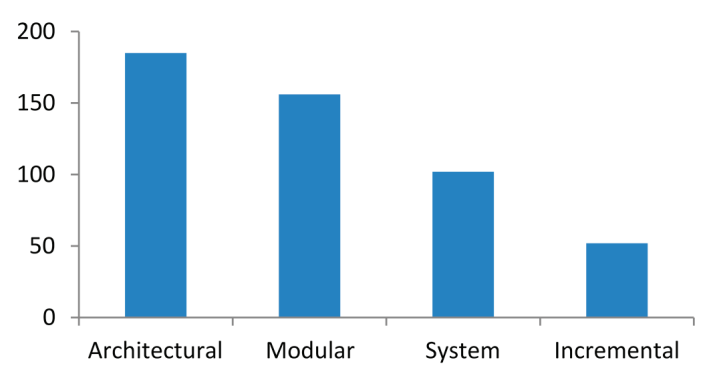

Figure 2. Innovation classification based on Novelty. 


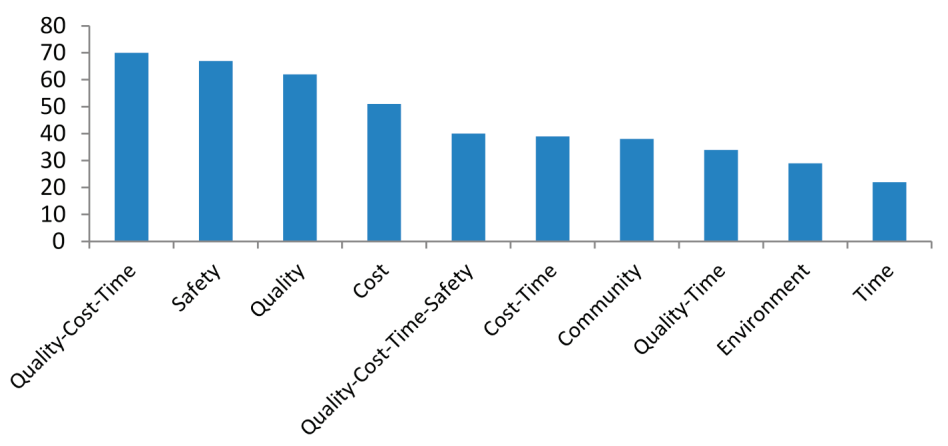

Figure 3. Innovation classification based on Benefit.

\subsection{Pairwise Analysis}

The data was also analysed to identify emerging trends that would provide more insight in to the relationship between the three dimensions of the innovation classification system. When looking at the spread of innovation based on a pair of two dimensions of benefit and novelty, an interesting trend emerged. As illustrated in Figure 4, it appears that architectural and modular categories of innovation are more focused on delivering a single benefit. On the other hand, system category of innovation seems to be the one that mostly delivers a combination of quality, time and cost benefits (see Figure 5). Modular innovation also appears to be the most prevalent category which focused on the indirect productivity improvements through safety, environment and community (see Figure 6).

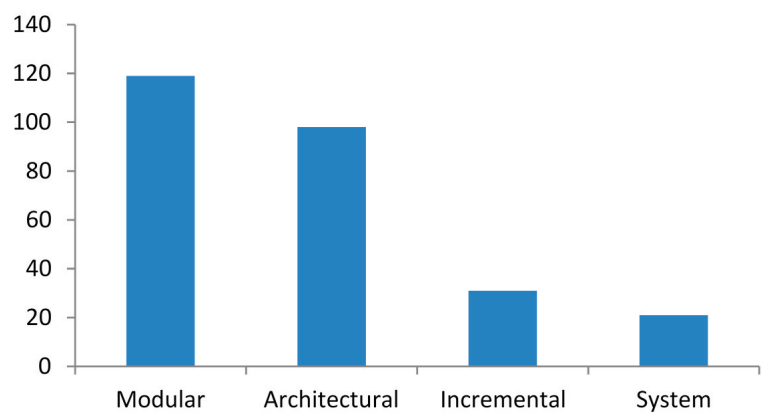

Figure 4. Innovation novelty categories that focused on delivering a single benefit.

When looking at the pair of innovation types and benefits similar trends appear. As illustrated in Figure 7, the majority of innovations are delivering a combination of direct productivity improvement benefits such as quality, time and cost, were from the two categories of design and method. On the other hand, function and tool categories seem to be more focused on delivering a single benefit (see Figure 8). Tools also appear to be the most prevalent type of innovation that deliver either safety, environment or community benefits (see Figure 9). 


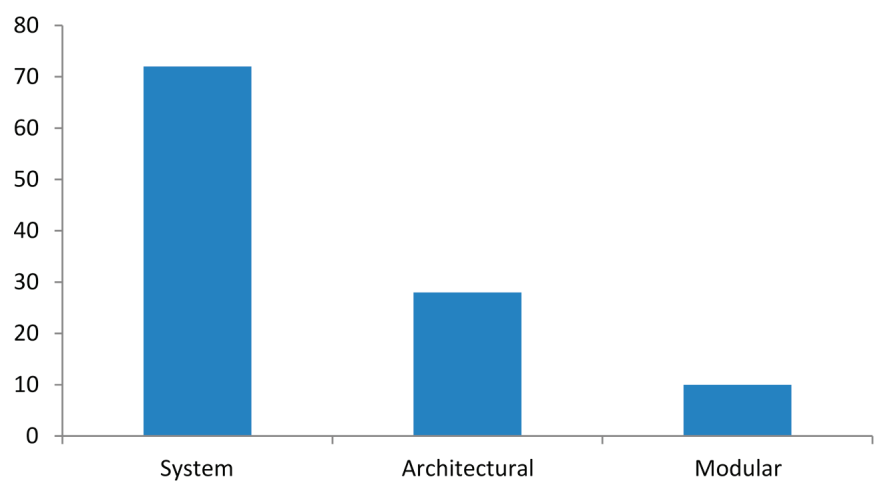

Figure 5. Innovation novelty categories that delivered a combination of quality-time-cost benefit.

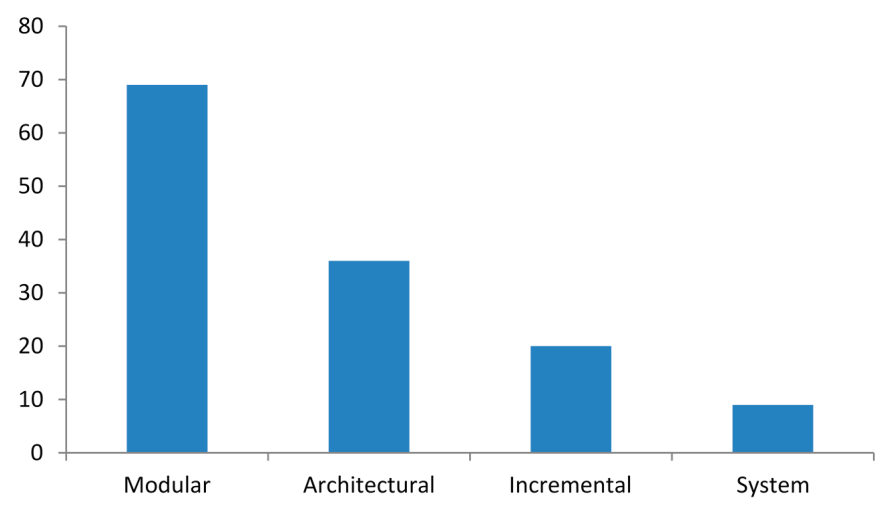

Figure 6. Innovation novelty categories that focused on either sustainability, safety or community.

Analysis of the pair of type and novelty also reveals some interesting trends. As illustrated in Figure 10, design innovation was dominated by architectural level of novelty, while product innovation was split between modular and architectural. Technology innovation (design + product) on the other hand was mainly dominated by system level of novelty. Modular and architectural innovations made up the majority of the tools and functions, while those innovations classified as method were mainly system or architectural innovations. Figure 11 also illustrates that the system level of novelty was mainly prevalent in methods, while modular innovations were mostly found under the tool and function categories. Architectural innovations were spread amongst tools, designs, functions and methods. Also, it was found that most incremental innovations were under the tools category. 


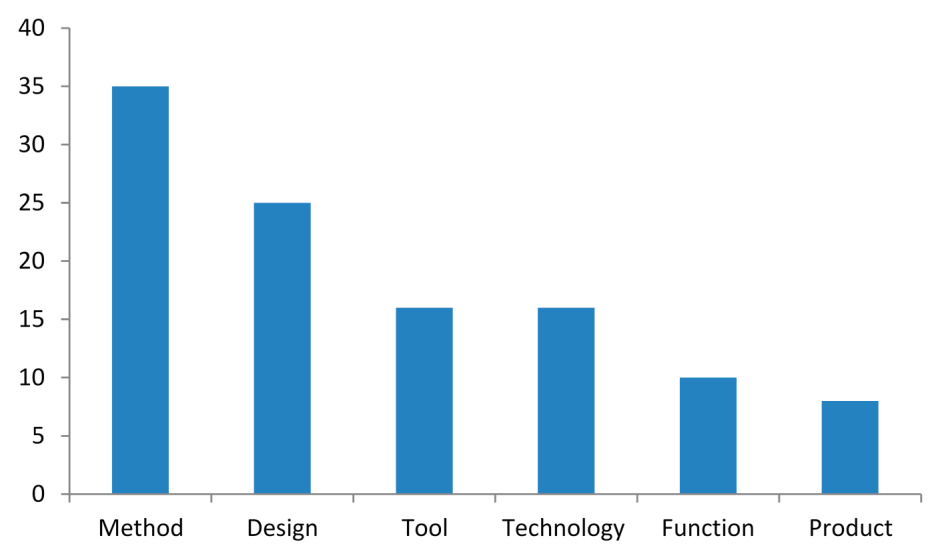

Figure 7. Innovation types that delivered a combination of quality-time-cost benefit.

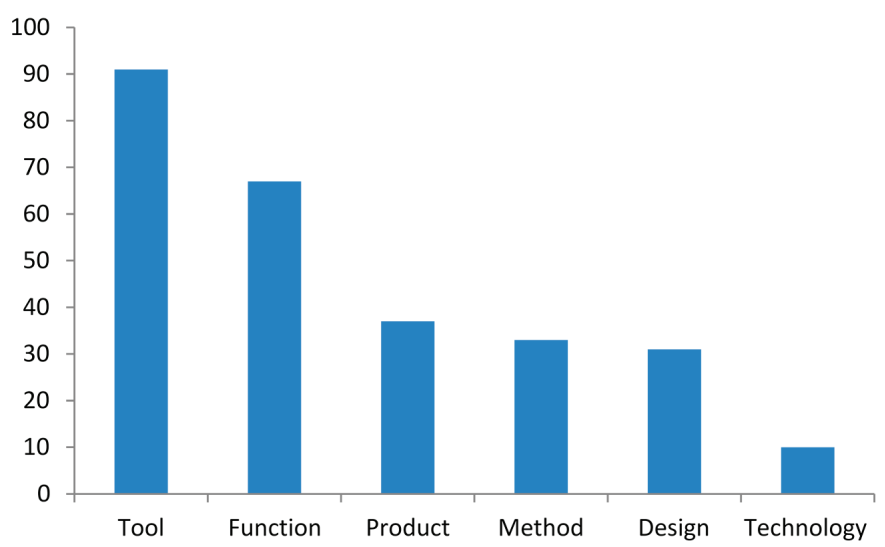

Figure 8. Innovation types that focused on delivering a single benefit.

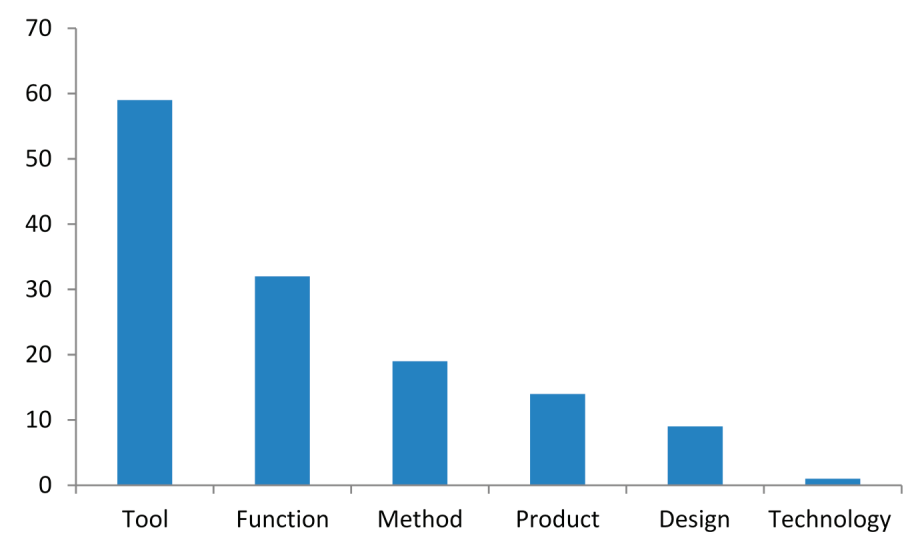

Figure 9. Innovation types that focused on either sustainability, safety or community. 


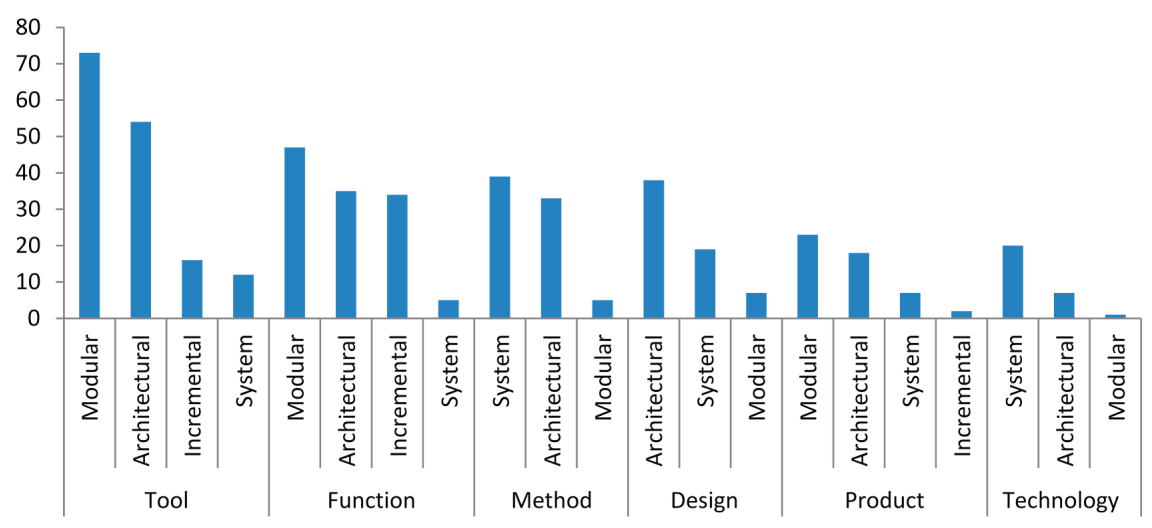

Figure 10. Degree of novelty of various types of innovation.

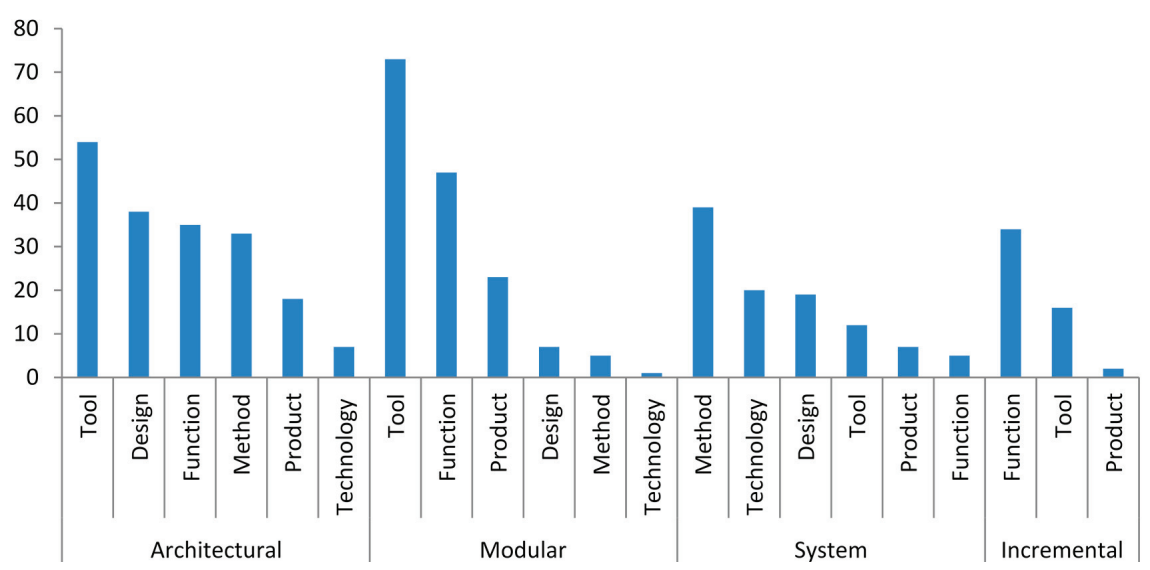

Figure 11. Spread of innovation types for each level of novelty.

\section{Discussions}

The results of the analysis of 500 reported innovations by the alliance group, SCIRT, clearly demonstrate the diversity of types, degree of novelty and performance improvement benefits among construction innovations. The trends presented in the previous section have all emerged naturally based on the organisational dynamics and culture present among the member organisations as well as within the virtual alliance organisation. Given that innovation KPI reporting was linked to pay/reward system for the member organisations, there was motivation for all parties to look for opportunities to innovate. However, the data shows that most of the reported innovations were tools or functions that were developed to overcome immediate problems facing the operational teams. As a result, most of these innovations were modular or architectural in terms of novelty. This indicates that most reported innovative solutions were developed to either solve localised problems or issues arising at the interface of operational sub-systems. The data also shows that when architectural and modular innovations were dominant, the reported innovations 
were mainly focused on a single aspect of performance improvement. In contrast, the results show that when more sophisticated types of innovation such as technology and methods were developed, the impact was more widespread and significant, delivering benefits along multiple dimensions of performance such quality, time and cost.

Given that the reported innovations in the SCIRT database were developed without consideration given to the types of innovation, degrees of novelty or performance improvement benefits, they were all treated the same. The KPI associated with innovation simply counted the number of innovations reported by the member organisations. The main reason for this equal treatment of the reported innovations is lack of in-depth understanding of various categorisations of innovation by the SCIRT management team. Consequently, they missed the opportunity to guide and direct the innovation efforts of the alliance member organisations towards specific types of innovation which could lead to specific aspects of productivity and performance improvement.

One important point with respect to future application of the classification system to other construction projects is the uncertainty associated with matching the definitions of the various categories of the classification system with the information provided about the particular innovation. Although this was not a major issue in the case of innovations in the SCIRT database, it is still a notable concern for future applications. While applying the classification system in the case study, it was clearly evident that this uncertainty was directly related to the amount and quality of information provided about the innovation. Given that in this case the classification system was applied retrospectively, the researchers did not have any influence over how data about the innovations was collected and reported. It is therefore suggested that if the proposed classification system is used for development of a KPI system, a data collection template is designed based on the definitions of the various categories. This could for instance take the form of a questionnaire with specific questions around novelty, benefits and type of innovation, which would ensure that adequate information is available for classifying the particular innovation.

\section{Conclusions}

In conclusion, the results of the analysis illustrate the need for a more in-depth understanding of innovation in construction. The innovation classification system presented in this study provides a practical tool for researchers and practitioners in the field of construction management, to not only better understand the relationship between innovation and productivity, but to also put in place mechanisms to influence the types of innovation developed in their projects with the aim of maximising their productivity performance. This classification system can be used by construction organisations and alliances in the future to develop more detailed methods of calculating innovation KPIs, based on the innovation type, novelty and benefits factors. In such methods, different weightings can be given to each factor as a mechanism to encourage certain types of innovation, with higher levels of novelty and focusing on specific combination of performance indicators that are tailored to the specific requirement of the construction project. For instance, in projects where the Green Construction approach is adopted, the system can be set up to encourage more environmental benefits, whereas if Prevention through Design (PtD) approach is utilised, it can be configured to emphasise safety related innovations in products and designs. Future research 
should look into expanding the application of the innovation classification system from productivity improvement to these emerging construction paradigms.

\section{Acknowledgments}

The authors would like to express their gratitude to Building Research Association of New Zealand (BRANZ) for providing the funding for this research. This work would not have been possible without access to the SCIRT innovation database. The authors thank SCIRT and University of Canterbury Quake Centre for providing access.

\section{Author Contributions}

Mohammadali Noktehdan: Paper writing, data analysis, development of classification system; Mehdi Shahbazpour: Paper writing, development of classification system; Suzanne Wilkinson: Paper writing.

\section{Conflicts of Interest}

The authors declare no conflict of interest.

\section{References}

1. Murray, M.; Murray, M.; Langford, D. Rethinking construction: The Egan report (1998). Constr. Rep. 2003, 1944-1998, 178-195.

2. Smith, B. Valuing the Role of Construction in the NZ Economy. Available online: http://www.constructionstrategygroup.org.nz/downloads/PwC\%20Report\%20-\%20Construction \%20Sector\%20Analysis\%20Final\%204\%20Oct.pdf (accessed on 30 March 2015).

3. Wilkinson, S.; Kempton, T.; Gleeson, A. Identifying Canterbury Rebuild Project KPI's-Baseline Report. Available online: http://buildingvalue.co.nz/sites/all/themes/productivity/images/ Wrap/Reports/1211Canterbury_KPI_Project_Nov12.pdf (accessed on 30 March 2015).

4. Reichstein, T.; Salter, A.J.; Gann, D.M. Last among equals: A comparison of innovation in construction, services and manufacturing in the UK. Constr. Manag. Econ. 2005, 23, 631-644.

5. Statistics New Zealand. Research and Development Survey. Available online: http://www.stats.govt.nz/browse_for_stats/businesses/research_and_development/inforeleases.aspx (accessed on 30 March 2015).

6. Seaden, G.; Guolla, M.; Doutriaux, J.; Nash, J. Strategic decisions and innovation in construction firms. Constr. Manag. Econ. 2003, 21, 603-612.

7. Loosemore, M. Innovate or perish? Exploring some of the myths of construction innovation. Australas. J. Constr. Econ. Build. Conf. Ser. 2014, 2, 44-55.

8. Huergo, E.; Jaumandreu, J. Firms' age, process innovation and productivity growth. Int. J. Indust. Org. 2004, 22, 541-559.

9. Koskela, L.; Vrijhoef, R. Is the current theory of construction a hindrance to innovation? Build. Res. Inf. 2001, 29, 197-207. 
10. Park, H.-S.; Thomas, S.R.; Tucker, R.L. Benchmarking of construction productivity. J. Constr. Eng. Manag. 2005, 131, 772-778.

11. Brown, M. Introduction to Innovation-Managing Ideas into Action; Henley Management Center/Price Waterhouse: London, UK, 1994.

12. West, M.A.; Altink, W.M.M. Innovation at work: Individual, group, organizational, and socio-historical perspectives. Eur. J. Work Org. Psychol. 1996, 5, 3-11.

13. Organisation for Economic Development. Oslo Manual: Guidelines for Collecting and Interpreting Innovation Data; OECD Publishing: Pairs, France, 2005.

14. Shahbazpour, M. Strategic Manufacturing System and Process Innovation: A Framework for Small and Medium Sized Enterprises. Ph.D. Thesis, The University of Auckland, Auckland, New Zealand, February 2010.

15. Slaughter, E.S. Models of construction innovation. J. Constr. Eng. Manag. 1998, 124, 226-231.

16. Bassioni, H.A.; Price, A.D.F.; Hassan, T.M. Performance measurement in construction. J. Manag. Eng. 2004, 20, 42-50.

17. Environmental Protection Agency. The Lean and Environment Toolkit. Available online: http://epa.gov/lean/environment/toolkits/environment/resources/LeanEnviroToolkit.pdf (accessed on 30 March 2015).

18. Tatum, C.B. Classification system for construction technology. J. Constr. Eng. Manag. 1988, 114, 344-363. 


\title{
A Production Model for Construction: A Theoretical Framework
}

\section{Ricardo Antunes and Vicente Gonzalez}

\begin{abstract}
The building construction industry faces challenges, such as increasing project complexity and scope requirements, but shorter deadlines. Additionally, economic uncertainty and rising business competition with a subsequent decrease in profit margins for the industry demands the development of new approaches to construction management. However, the building construction sector relies on practices based on intuition and experience, overlooking the dynamics of its production system. Furthermore, researchers maintain that the construction industry has no history of the application of mathematical approaches to model and manage production. Much work has been carried out on how manufacturing practices apply to construction projects, mostly lean principles. Nevertheless, there has been little research to understand the fundamental mechanisms of production in construction. This study develops an in-depth literature review to examine the existing knowledge about production models and their characteristics in order to establish a foundation for dynamic production systems management in construction. As a result, a theoretical framework is proposed, which will be instrumental in the future development of mathematical production models aimed at predicting the performance and behaviour of dynamic project-based systems in construction.
\end{abstract}

Reprinted from Buildings. Cite as: Antunes, R.; Gonzalez, V. A Production Model for Construction: A Theoretical Framework. Buildings 2015, 5, 209-228.

\section{Introduction}

The construction industry faces ongoing challenges. From an external perspective, economic uncertainty, increased competition within the industry and the growing influence of regulatory agencies drive profit margins down. From an internal viewpoint, the increase in the numbers of features in a project's scope, against shorter construction deadlines and restricted budgets raise the complexity of building construction projects. Additionally, building construction often occupies the bottom of industrial productivity rank reports worldwide [1], even showing negative productivity rates [2]. The construction sector relies on management practices based on intuition, experience and poor risk management. These practices often preclude the appropriate level of ability to handle the uncertainty and complexity [3,4] involved in construction projects, resulting in project failures in terms of finishing projects within deadlines and budgets [5]. As a common practice, the building construction industry utilizes ordinary project management practices and frameworks. Practices are limited to assessing the consequences of deviations from the project plan rather than dealing with the causes of delays in the production system [6]. "Heisenberg's principle indicates that the conventional construction management practice of measuring the overall timeliness and budgetary status (position) of a project using schedule/cost variances will result in a less precise assessment of the throughput (momentum) of the project" [4]. At the tactical management level, little attention has 
been paid to production management in building and construction [7]. Traditional scheduling approaches in construction, such as the critical path method, have been used unrestrictedly, producing unfinished and erratic plans [4]. Even more, a developed estimation practice, such as the line of balance, fails to deliver a reliable outcome. Instead of being derived from manufacturing practices, the line of balance was purely copied regardless of the inherent production differences between manufacturing and construction projects. Both approaches assume that construction processes are constant with some degree of variability, setting constant estimation values for production and adding an extra measure to accommodate variations. In summary, the dynamics of the production system is overlooked [8]. In fact, the building construction industry has no history of applying mathematical approaches to model and manage production [9]. Mathematical models have enabled a comprehensive understanding of production mechanisms [10]. For instance, manufacturing has a record of successfully developing and using robust mathematical models to improve productivity, system comprehension and event forecasting [11,12]. Nevertheless, the manufacturing model does not directly apply to construction. Steady-state conditions, nonterminating events, long production runs, medium to low levels of uncertainty and permanent production facilities characterize production in manufacturing [12]. Production in construction takes place under a mix of steady- and unsteady-state conditions, with terminating events, short production runs and various levels of uncertainty, all conducted in temporary production facilities [13-15].

The impacts of World War II in Japan restricted market demand and the production model. Mass production changed to the production of a high variety of products in small quantities. Moreover, in order to adapt to the post-war scenario of the financial incapability of acquiring new machinery, the Japanese industry had to work with the existing production equipment in a more efficient manner. The new diversified small-batch production and post-war recession pushed the industry to re-evaluate the use of resources and production methods and resulted in a shift in production paradigms in the manufacturing industry. Consequently, a new production model should be developed to increase productivity while using existing resources. The production philosophy named just-in-time (JIT) together with automatization established the foundations of the Toyota Production System (TPS) in 1945. TPS is a compilation of observations from Taiichi Ohno and Shigeo Shingo and driven by Toyota's goal of "catching up" with the American automotive industry. TPS only received notable exposure during the oil crisis in the autumn of 1973, when the improvements brought by the relentless elimination of waste helped Toyota through the Japanese economic crisis [16]. In an attempt to reproduce the success achieved by Toyota, the automotive industry adopted TPS as a production model and then forged a new manufacturing philosophy, later entitled "lean manufacturing" [17]. The lean principles-waste eradication, flow, value, value-stream and zero-defect — crossed the boundaries of manufacturing. These principles permeated several productive systems and industries, such as software development [18], even reaching business models [19]. At the same time, manufacturing was formulating mathematical explanations of production management. Studies about the variables of the productive process - a process is a repetitive collection of interrelated tasks aimed at achieving a specific goal-provided a quantitative meaning to production management practices in manufacturing. Equations could now represent queueing systems [10], lead time [20], machine maintenance [21], Kanban [22], 
inventory management [23], throughput, cycle time and work in progress [11]. The set of equations enabled a deeper understanding of the current practices and the outcomes of their interaction.

The building construction industry has also demonstrated interest in lean principles [7]; later named lean construction [24]. The transformation, flow and value generation views associated with a set of principles constitute the transformation-flow-value (TFV) theory of production. However, the implementation of lean principles has been sporadic, rather than an industry standard [25]. Several contradictions to the lean values, such as excessive consumption of raw material [26], disconnection of activities, preventing the establishment of a flow [7], a focus on costs over value [27], an inefficient measurement system [28], high levels of rework due to production errors or changes in technical specifications [29] and worker safety [30], still populate the list of the most common problems in construction endeavours. Inefficiency at the tactical level is only one barrier to the adoption of manufacturing principles. Intrinsic project characteristics also increase the challenges to the industry. Uniqueness - each project produces a distinct product, service or result with individual features of the project work [31] - temporality — "indicates that a project has a definite beginning and end" [32] — and uncertainty [32-34] are some attributes that set apart projects and manufacturing. As a matter of fact, even repetitive construction projects - projects characterized by repetitive activities, as, for instance, pipelines, tunnels, mass housing projects and high-rise buildings [35,36] — are exposed to the effects of uniqueness, temporality and uncertainty. Despite an insufficient pace to "catching up" with market demand, construction has progressed towards the incorporation of TPS practices [7] and an understanding of its production.

Construction has successfully comprehended the effects of variability in production [37] and the influence of buffers on sequential activities [38]. Researchers consistently advance the design and management of work-in-progress buffers. Additionally, approximate models can be obtained by approaches, such as discrete simulation [39], fuzzy logic [40], neural networks [41], statistics [42] and control theory [43]. However, the results are still theoretical and restrictively applied [15,44,45]. Moreover, obtaining mathematical expressions that quantitatively explain the production mechanisms of different repetitive construction projects has been a challenge $[15,46]$ and is still to be formulated. Much work has been carried out on how manufacturing practices apply to construction projects, mostly lean principles. Nevertheless, there has been little research to understand the fundamental mechanisms of production in construction. The current knowledge is at a rudimentary stage and has not yet fully established the foundations of the production model in construction projects. This research entails an in-depth literature review to examine the existing knowledge about production models and their characteristics in order to establish a foundation for the production model in construction. This literature review does not intend to pursue lean construction or its production models. This study searches outside the construction scenario for best practices in production and then inside for the barriers to the implementation of these practices. The study retraces production characteristics from manufacturing that are applicable to building construction projects in order to obtain a similar system view that propitiates the development of manufacturing production laws, such as variability, conservation of material and capacity laws [11]. Furthermore, the concepts are reviewed from a microeconomic/strategic/tactical vantage point required for project management development, regardless of the project technical specificities. As a result, a theoretical framework, 
which may serve as a basis for the further development of a mathematical production model aimed at predicting the performance and behaviour of dynamic project-based production systems in construction is proposed.

\section{A Theoretical Framework}

\subsection{A Brief Understanding of Construction}

The understanding of what is construction extrapolates its technical characteristics. In this research, construction is not restricted to civil engineering and architecture, but comprehends a broader understanding of building, putting up, setting up, establishing and assembling. Construction is the materialization of a concept through design, taking into account functional requirements and technical specifications for a project product utilizing specialized labour. In other words, it is the creation of a product that will fulfil a strategic goal. This study excludes prototyping as the final product; however, a project scope may include prototyping as a deliverable or stage, as, for example, in the design phase. Prototype objectives test a process or concept in real situations and then provide information about what works and what needs improvement before the final product. In contrast to prototypes, the project product is final and definitive. Project products, according to the design specifications, have usability in a first and unique building. Project products of building construction are, for instance, software, pipelines, roads, bridges, tunnels, house building and oil well construction. In summary, project deliverables with different technical backlogs and fields of application are in accordance with the definition of construction stated in this study. Moreover, the existence of a technical backlog brings a dimension of repetitiveness. Because these projects require specialized labour, the project executive is likely to be involved in projects with a similar technical background. For instance, an offshore oil well construction company is more likely to have future endeavours in the same technical field rather than in a road building. In this case, the repetitiveness occurs horizontally across different projects with a similar scope. A project can also have repetitiveness within an endeavour: for instance, the construction of similar products, such as housing units, floors of a skyscraper or the installation of cases in an oil well. These processes illustrate a vertical dimension of repetitiveness, i.e., process repetition in a unique project.

In order to understand how construction applies to different fields and where construction is placed in project life cycles, ten different project management frameworks were analysed. Figure 1 displays the organization of several project-driven approaches in four clustered stages grouped by fundamental characteristics. The initial stage (feasibility) consists of a group of processes aimed at identifying the best possible solution to achieve the strategic business goal intended by the project sponsor. Additionally, these processes should determine if the project product corresponding to the selected solution can actually be built or created (several applicable practices can be found in [47]). The second stage (design) presents a group of processes where the project and technical teams examine the outcome of the feasibility stage. The teams produce design specifications and project plans that contain the information about how to make a suitable project product based on the best possible solution. The next stage (construction) is to build the product following the technical instructions and executing the management plans from the previous stage. The final step 
(operation) is delivering the finished product to the user, who will use the project product to generate the benefits and consequently achieve the benefits of the business goal [34]. Operation is not considered a project stage, although the beginning of this stage commonly determines the termination of the project life cycle [31].
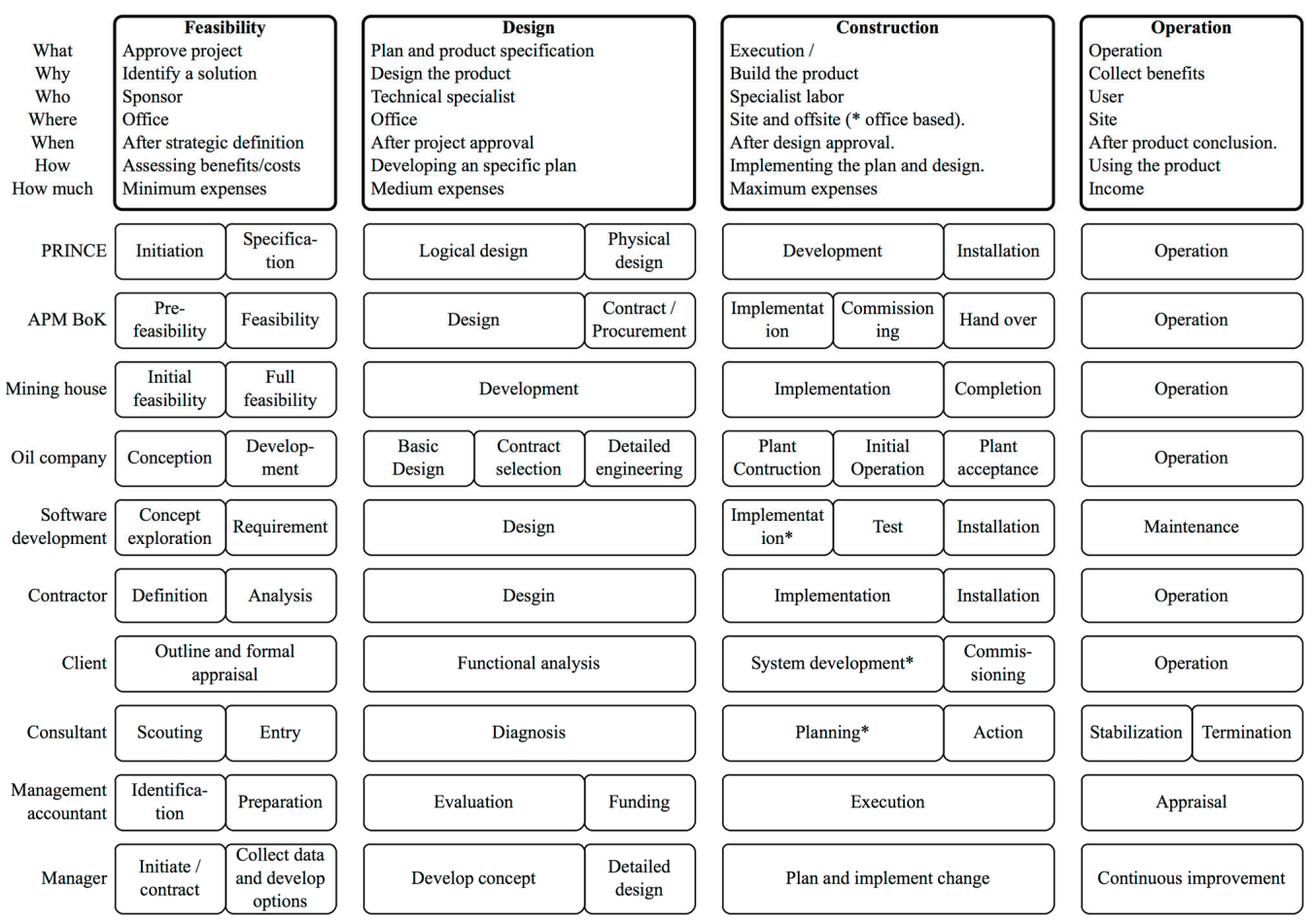

Figure 1. Characteristics of project stages (projects stages of various approaches adapted from Smith, Merna and Jobling [48], originally from McGetric, The Project Life Cycle, 1996). For additional information about the Project in Controlled Environment (PRINCE) and Association for Project Management Book of Knowledge (APM BoK), refer to [34] and [49], respectively.

\subsection{Production Facilities}

The project takes place in different locations through the three project stages (feasibility, design construction) and is frequently related to where the main phase stakeholders are located. First, it originates from strategic demand, and feasibility studies take place where the sponsor is located. At this stage, the management has recognized that normal operation is unable to address a particular business requirement [50]. Consequently, the management sponsors a unique endeavour to address this outstanding business requirement, initiating the project. Once initiated, it is up to the sponsor to choose the best solution and investment to solve the issue. Altogether, the feasibility stage is likely to occur as an internal business routine. 
Project and product design require strong technical knowledge, which is usually not the sponsor's forte, since the initial motive for the project's existence is the inability of routine operations to accomplish a specific business requirement [50]. Hence, it may be necessary for a specialist third party to join the project and develop the technical features, collect all requirements, and plan and design the project product to execution. For this reason, the design stage tends to move from the project sponsor's office to third party offices with regular interaction between stakeholders. Thus, the influence of stakeholders is less than at the previous stage [51].

Similar reasoning about technical development is valid for the construction stage, where each task of the construction plan often requires either a specialist in the trade or a contractor. For this reason, work packages are broken down and distributed to several independent third parties. Frequently, these third parties are only connected to each other by the current project, without a prior collaboration background between the companies or teams involved. The location, where the final project product takes place, is undoubtedly a strategic decision. Nevertheless, the work packages of the project may be produced in different facilities, on-site or off-site. The work packages are spread and distributed to suppliers according to the project procurement management plan [25] and are, in general, produced in facilities subject to the supplier's business model, rather than the project owner's choice. Again, the influence of stakeholders is diminished [51]. The production facility is distinctively important, because this stage gathers most of the resources and labour needed for building the project product as designed and planned. This resource convergence is responsible for the majority of expenditures in the project [25,31], and it reveals any faults and inaccuracies of the plan developed in previous project stages.

Clients perceive several benefits of off-site production over on-site [52]. Benefits, such as production time, quality and safety, are often mentioned. Interestingly, the most important benefit of off-site production is not being executed on-site. In the rank of benefits, according to importance and likelihood, the top three answers refer directly or indirectly to the reduction of activity on-site. The benefits from the highest to lowest rating are: minimize on-site operation; reduce congested work areas; and multi-trade interfaces to minimize on-site duration [52,53]. Construction's on-site production implies challenging logistics. The location of a construction site solely depends on the final user needs, which are different from manufacturing, where the choice of factory location fulfils strategic logistic goals of product distribution and supplier connection. From a logistic perspective, "the delivery is to a temporary location, without permanent facilities for handling material" [14]. The provisional storehouse complicates the employment of optimal delivery routes and connections to preferred suppliers, further hindering the formation of an efficient supply chain. Location may severely interfere with the supply chain. Depending on the construction site location, several nontechnical restrictions may apply. For instance, building a skyscraper in the city centre may require obeying municipal restrictions. Timetables and regulations for moving load, material delivery and dispatch and also additional safety procedures due to population proximity [54] may be necessary. On the opposite side, construction in isolated locations endures materials transportation, because of the long distances from suppliers and improper or even inexistent transportation routes [55]. A long distance from preferred suppliers often raises costs to a level where the chain becomes unfeasible, forcing executors to work with alternatives sources. New 
parties are most likely to experience a slower project learning curve than the usual partners, because previous involvements support the creation of both product and process know-how via lessons learned.

For the building construction industry, the supply chain is critical due to the excessive amount of raw materials required for product assembly [56]. Expressly in the construction phase, the raw materials need to arrive on-site in the correct amount and sequence, since there is a minimal warehouse installed on-site, which is incapable of handling massive amounts of material [57]. Arriving and queueing coordination is essential to not overload the warehouse nor starve the production line. Material surplus may impact how material flows to the production line, because overstocks may delay picking activities, further reducing the necessary space for cargo handling inside the warehouse. Starving the production line is more critical, since it may switch off the production line or part of it.

\subsection{Production Run}

By definition, the short run is the window frame when steady factors drive production. Moreover, the addition of capital is not possible, so increased output requires more labour [58]. Scope, budget and schedule constraints are core characteristics of the production of projects [31], i.e., steady factors that restrict the amount of work, the addition of capital and the project execution time frame. On a short production run, as in projects, the deadline, budget and total number of units to produce (scope) are clear from the beginning. Although clearly and explicitly stated in the project scope, the estimations of these constraints are, unfortunately, not always realistic. In contrast, a long production run is unclear about both the production end and total units to produce, thus relying on forecasts and market conditions [59]. For instance, upstream oil production rig expects, based on the oil prospect, to extract a set number of oil barrels in a set time. This notwithstanding, the extraction from the reservoir will continue until the extinction of oil or until its operation becomes unfeasible.

Consequently, the average cost (cost/units) [59] for a short production run is more accurate than for the long run, because the units to be produced can be considered constant. Initially, the long run (forecasted units) has a higher potential risk than the short run (exact units) for the same total cost. However, the long run may produce more units than the forecasted number, creating a more favourable cost/units relation [59]. Moreover, the short-run production aggregates higher costs per unit than long-run production, because the number of units to produce is much smaller than on that for long-run production. This value concentration makes short-run production more sensitive to risk in the case of unit variation. In other words, the expected value is higher because the risk impact is higher for the same risk probability (expected value $=$ risk probability $\times$ impact) [60]. In summary, short-run production has a lower potential risk, but requires more accuracy, because value concentrates in the production units. Consequently, small variations in labour may affect production and the overall cost. 


\subsection{Event Termination}

Projects by definition are finite terminating endeavours [31,34]. There are some divergences in the literature $[31,33,34]$ about when exactly a project starts, although the termination is clear. The handover to the user sets the final milestone of product construction, marking the project's end. Any activities later on relate to project documentation and formal closure. Considering every stage, deliverable or activity startup and ending, the system (in this study, the project) receives several sequential on and off switches. These interruptions cause work fragmentation [42]. This fragmentation implies numerous transient states, i.e., a timeframe in which the system is either adapting to a state change or responding to a disturbance, e.g., after the impact of a risk. Such variation may affect the state condition of production, changing the production state from a steadyto unsteady-state condition.

\subsection{State Condition}

Projects involve a unique state change [61]. During project execution, the amount of work is variable and concentrated on the "carrying out the work" stage [31]. Consequently, initial and final project stages are low-speed regions. The project velocity $[31,62,63]$ increases from zero, at project start, accelerating to its maximum at the execution stage and then slowing down to a full stop at project end. However, the velocity variation is not the determinant of the state condition of the system. The variation, or not, over time of the relation of different properties that generate the system output from the input set the state condition of that system. Therefore, the system is in a steady-state when several properties are invariant over time [64]. Once these properties are invariant, the steady-state condition gives a means to the development of simple mathematical equations. For instance, in the steady-state, average work output is strictly less than average capacity, as claimed by the law (capacity) in manufacturing [11]. The law (capacity) does not directly apply to projects. Projects have a distinctive outcome, involving different teams with different technical knowledge and learning curves [65]. Regarding the production system of the project, these properties alter the output/input relation over time, which is the main characteristic of unsteady-state systems [15]. As a consequence, projects assume the unsteady-state condition. However, parts of the project-stages, deliverables, activities or processes - might assume a steady- or unsteady-state depending on process repetitiveness [13], variability and the conservation of process properties over time.

\subsection{Complexity}

Vastly present in the literature, the word "complex" seems to stand for a supernatural force supposedly responsible for disturbances, a scary ghost haunting projects. With no absolute definition of what complexity means, the only consensus among researchers is that there is no agreement about the specific definition of complexity [66]. However, a characterization of what is complex is possible. A structure is complex; if composed of several interconnected pieces [67], with dynamic networks of interactions, and their relationships are not aggregations of the individual static entities [68]. A theory derived from chaos theory follows a similar principle: "The complexity theory states that critically interacting components self-organize to form potentially 
evolving structures exhibiting a hierarchy of emergent system properties" [69]. Both statements point to the interaction between the internal structures as complexity characteristics.

Projects are complex. Projects involve several interrelated stakeholders, deliverables [70] and activities [71] in order to deliver a strategic state change $[61,72]$. Given that, projects, in a broad definition, show the characteristics of what is complex. Methods to assess, classify and measure project complexity have been discussed in the literature [69,73-75]. Most methods are restricted to computations involving scheduling and activity relationships [73,76,77]. Although these models provide a relative comparison of the complexity of similar projects in the same industry, they fail on a broader scale. The analytical hierarchy process (AHP) [78] provides a more inclusive and simple approach to complexity evaluation. AHP can assess a set of pre-chosen project criteria of a variety of projects, providing a calculated numerical grade from zero to one for complexity comparison [74]. Despite the fact that it is possible to use AHP to congregate projects into clusters, such a study does not exist. As a consequence, no explicit information comparing the complexity of projects in building construction with other industries could be traced.

\subsection{Uncertainty}

Similarly to complexity, there is no consensus about a pure definition of uncertainty. Uncertainty is an unintelligible expression without a straightforward description. Keynes was the first economist to incorporate uncertainty as a theory pillar, adding a factor of distrust to economic science. Keynes' studies explored how to be rational in an uncertain world, in what degree and how to specify uncertainty (refer to Mello [79] about Keynes' life and work). Later, Keynes studied the nature and effect of uncertainty on statistics. "Human decisions affecting the future, whether personal or political or economic, cannot depend on strict mathematical expectation since the basis for making such calculations does not exist" [80]. In the final analysis, uncertainty is beyond any prediction, forecast, calculation or measurement notably when considering human behaviour [49]. The main reason for the existence of a project is to achieve the value perceived by the stakeholders $[33,34,49,61,72]$. Nevertheless, value is relative [65]. Therefore, internal and external, direct and indirect stakeholders may have a different perception of the achieved project business outcome depending on their vantage points and individual backgrounds [17,65,81]. That, according to Keynes, is a fertile soil for uncertainty. Inevitably, projects are conducted in an uncertain scenario.

\subsection{Risk}

The viewpoint of uncertainty and risk vary from different disciplines [82]. However, uncertainty is not risk [83]. While uncertainty is a potential, unpredictable, unmeasurable and uncontrollable outcome $[83,84]$, risk is a consequence of action taken in spite of uncertainty [85]. As an illustration, two men are about to skydive from an airplane, but there is only one parachute on-board. At this point, the level of uncertainty experienced by the men is equal, as to whether or not the parachute will open. The moment that one man chooses jumping, the uncertainty is the same to both, and he bears all the risk. The uncertainty ceases when the parachute opens during the skydiving, through time passing, event and action. Nevertheless, there is still risk regarding a safe landing even after 
uncertainty about the parachute opening has come to an end [85]. Similarly, in projects, the decrease of uncertainty and risk through time passing applies [86].

Projects are risky. The action of conducting an endeavour upon uncertainty is the intrinsic nature of risk $[83,84]$. Hence, dealing with risk is crucial in project management to achieve successful business results [31]. Controlling uncertainty is impossible [80,83]. However, it is possible to identify, measure, and formulate plans to transfer, provoke, mitigate, avoid or embrace the impact of risk [84], i.e., manager risk. Risk management's objective is to assure uncertainty does not deviate the project from the business goals [84]. In detail, risk managers triumph by maximizing opportunities, minimizing threads and hedging against the risk of a contingent, uncertain loss [87]. The primary manoeuvring instrument against uncertainty is the contingency plan [88], whose determination counts on the summation of expected value [60]. The contingency plan consists of the probability of risk occurring multiplied by the financial impact if it occurs [60] plus an amount judged sufficient to handle unforeseen risks. In addition to the contingency plan, calculating how the contingency could spread over the project lifespan is paramount, especially in projects with a high concentration of events, such as construction projects. Value-at-risk (VaR) "describes the quantile of the projected distribution of gains and losses over the target horizon" [87]. In other words, VaR forecasts the expected value distributed across the project life, so the expected value can be used as a buffer to possible variations, positive or negative, in the project plan.

\subsection{Variability}

Variability is a measurement of variation, i.e., the difference between results. Variability expresses a dataset that is clustered [89]. Risk impact causes variability, as more risk motivates a broader span of "potential scenarios of outcomes" [85]. The impacts of variability in construction production processes are well known and proven as a decrease in productivity [37], scheduled delays and cost overrun [38]. Considering short runs, the manipulation of the production output requires the administration of labour as an input. Hence, the understanding of the relation between labour and productivity potentially enables the control of production. However, correlating variability to labour is challenging. In statistics, the productivity of labour in building construction does not fit a normal distribution; provided that the variance is undefined, the curvature is leptokurtic [90]. Leptokurtosis may influence the productivity of labour analysis, while estimations made using a normal distribution overestimate at low levels of significance and underestimate at high levels of significance. The wider end of a leptokurtic distribution means risk is coming from outlier events, and extreme observations are likely to occur.

Projects are extremely exposed to variability caused by risk. On the one hand, the short-run production nature of projects requires the manipulation of labour input to control the output [91]. Labour is a prominent human factor in construction projects [42] and a primary source of uncertainty [80], which translates into an increase of risk probability. On other hand, the short-run production concentration of the average cost contributes to an increasing of risk impact. Consequently, both factors - probability and impact — of the risk expected value are affected. In conclusion, at the occurrence of a risk event, a higher expected risk value implies a higher difference between the plan and the actual result, i.e., higher variability. 


\subsection{Cone of Uncertainty}

Uncertainty not only decreases over time passing, but it also diminishes its impact by risk management, specifically by decision-making. In other words, the impact of uncertainty strongly depends on the decision of the risks taken. Researchers' findings indicate that the project estimation builds on foreseen quantities of uncertain events distributed at several stages, resulting in the process of resolving decisions [92]. Figure 2 shows a decrease in variability and the consequent increase in the accuracy of estimates over project progression. Still, the cone does not form itself naturally. It is a product of various decisions made as the project progresses concerning the plan and product. Narrowing the cone implies removing sources of variability sooner. On the contrary, a wider cone results from later decisions [92]. Figure 3 presents a parallel analysis illustrating the impact of decisions made in early and late stages on possible cost reduction in construction projects. Hence, to protect subsequent events from variability and its effects, decisions should be made in early stages to narrow the range of possible later events that will impact the project and cause variability.

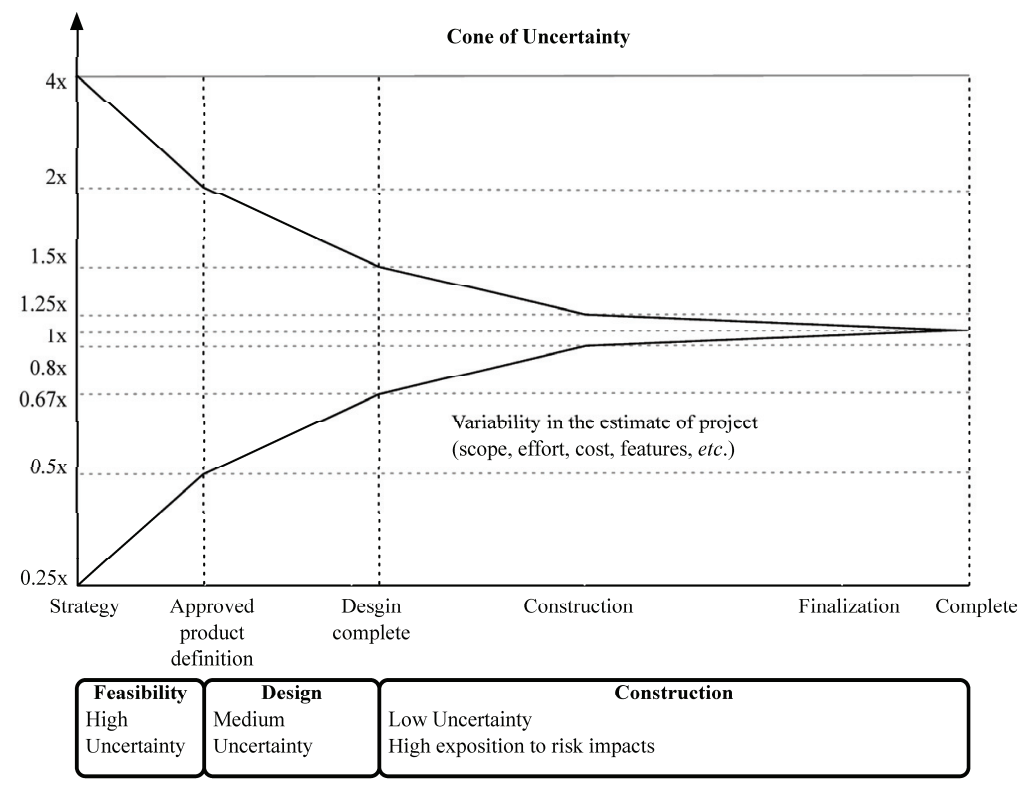

Figure 2. The cone of uncertainty for sequential project development (adapted from McConnell [92]).

Experience from past projects supports decision-making. Commonly called "lessons learned" by project literature and practitioners, the term defines the practice of learning from successful and unsuccessful past events. Insights from assessing captured outstanding project events in post-project reviews can dramatically narrow the cone of future projects. Despite having a remarkable potential to reduce variability in a project, in practice the "lessons learned" process frequently ends with capturing the outstanding events [91], dismissing further steps. The likelihood 
of reusing lessons learned depends on their applicability to future projects and the similarity between projects, in other words, project receptiveness.

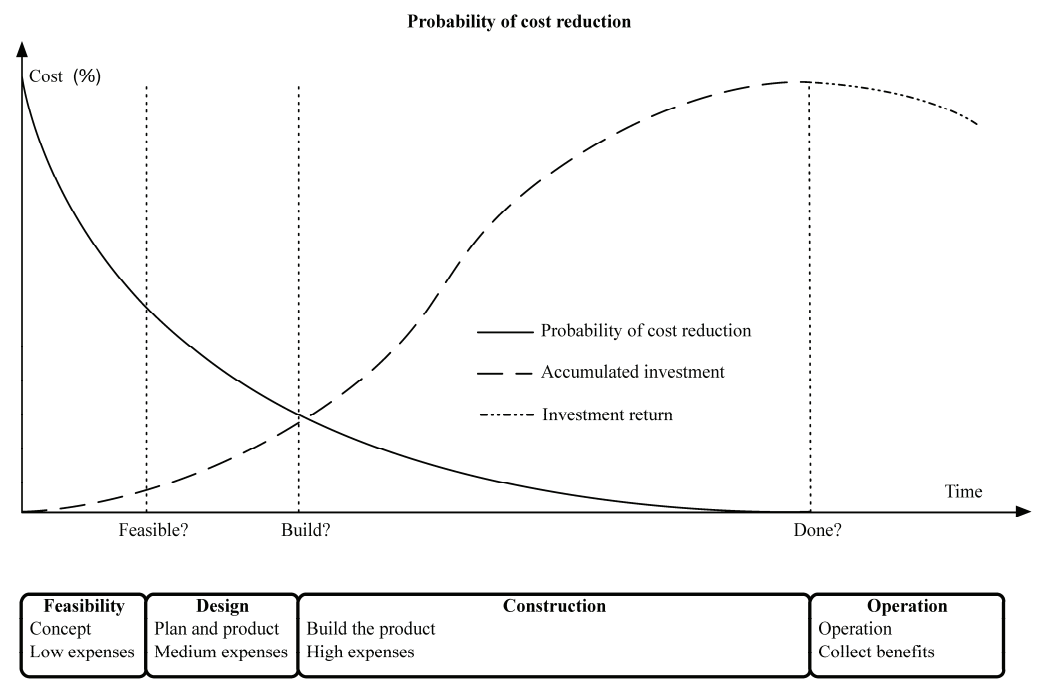

Figure 3. Graph of percentage cost over time demonstrating how early correct decisions may impact cost reduction (adapted from Smith et al., [50]).

\subsection{Repetitiveness}

"Repetitive manufacturing is the production of discrete units in a high volume concentration of available capacity using fixed routings. Products may be standard or be assembled from standard modules. Production management is usually based on the production rate" [93]. Construction produces multiple products in low volume coupled with the process structured in the midway of the batch, matching the major stages of product and process life cycles from Spencer [93]. A construction production run is short and easily countable considering the final project product. Nonetheless, the project product or a deliverable may be broken into several units, creating an artificial repeatability. For instance, the installation of the windows on the World Trade Center One (or Freedom Tower) is unique and not repeatable. However, this deliverable can be subdivided into floors. Therefore, instead of one unique product, there are 94 repetitions of the same product of "windows installed by floor". The deliverable can be subdivided even more. If each of the 21,800 windows to install is considered, the window installation process can be interpreted as very repetitive [94].

Repetitiveness provides the opportunity for greater use of lessons learned, inevitably proportionating improvement in processes and products. A high level of repetitiveness may propitiate continuous enhancements due to the product likeness, ease of measurement and comparison between construction cycles [35,93]. Formed by four basic sequential activities, plan-do-check-act (PDCA) — also known as Shewhart's cycle — founded an unceasing circle of quality enhancement [31]. Likewise, Six Sigma, a set of techniques and tools for process improvement, aims 
for defect riddance and variability minimization [95]. At the sixth sigma level, processes in a state of statistical control expect no more than 3.4 defects per million opportunities. Additionally, the short-term process capability index (Cpk) is equal to two. These values typify a stable process almost free of variability. Consequently, processes at the sixth sigma level are steady state [96]. Measurement of Six Sigma levels can provide a useful form to qualify the state condition of the process. Higher Six Sigma levels correlate with a process at steady state (low variability), and lower six sigma levels correlate with the unsteady state (high variability). The levels of Six Sigma can be accurately calculated [96], as can the process variability. It is possible to establish a mathematical correlation between these variables, quantifying the state condition of a process through its variability. In summary, repetitiveness creates the opportunity for improvement based on the use of lessons learned, PDCA or Six Sigma and variability reduction through decreasing uncertainty and narrowing the cone of uncertainty earlier. In terms of system description, repetitiveness creates a feedback connection with activities or processes [11]. Furthermore, repetitiveness defines a closed loop system where deviations of past events may be used as input to correct and control [11] current and future system output.

\section{Construction Project System}

Several elements found in this literature review connect the characteristics of construction projects to the characteristics of a dynamic system. As shown in Figure 4, the interconnectivity is explicit between project stages, in the event that subsequent phases rely on the accomplishment and performance of previous ones. This dependent connection remains valid for divided $n$-substages or $n$-activities and also applies to the proposed framework. The dependence of processes and/or activities is well documented in the literature and well known by practitioners. An activity or stage may impair or favour a successive action depending on the level of correlation and dependence. The interdependence of activities forms a conduit to the propagation of unsure events. Potential risks captured through the entire project life may impact project execution whenever not properly treated, resulting in project deviations. This sequence of events is represented in the system by the flow of uncertainty to risk and the occurrence of risk events, through risk management filtering actions - avoidance, acceptance, sharing, transference, mitigation, motivation-and, finally, to variability. This flow resembles an intrinsic characteristic of systems in the presence of disturbance or noise. Control systems may transmit unfiltered noise across connections affecting vulnerable components and causing disturbances or unpredicted behaviour. Although the level of influence in this flow of sequential, parallel or overlapping relationships in the process or activity network have not been investigated at this point, understanding how risk transforms into variability, and especially how variability affects networked activities, propitiates an opportunity to develop methods aimed at avoiding and mitigating (filtering) the propagation of risk (noise). Regarding risk materialization in variability, different outcomes build on how concentrated or distributed the risk impact was. This scenario requires a function capable of scale variation and energy conservation (impact) when calculating the functional energy. The wavelet network evolved from the Fourier transformation: "wavelet network is a type of building block for approximation of unknown functions based on the concept of the multi-resolution approximation. The building block is formed 
by shifting and dilating the basis functions, the mother wavelet and father wavelet" [97]. A wavelet network may be used as universal function approximator ("a universal function approximator is a system that, given a set of predictor variables, can output an accurate estimate of some predicted variable" [97]) to estimate unknown nonlinear functions and to attain a required control performance. A new concept in the control area, wavelets have been successfully used in several applications, such as physics, signal processing and statistics, where small complicated details matter [98]. Operating on possibly the same conditions of wave theory-linear/nonlinear, deterministic/stochastic, time-domain/frequency domain, direct/inverse problems, discrete/continuous models [99] — control theory may create a proxy theory to explain the effects of variability in construction projects by extending the elements of the dynamic systems.

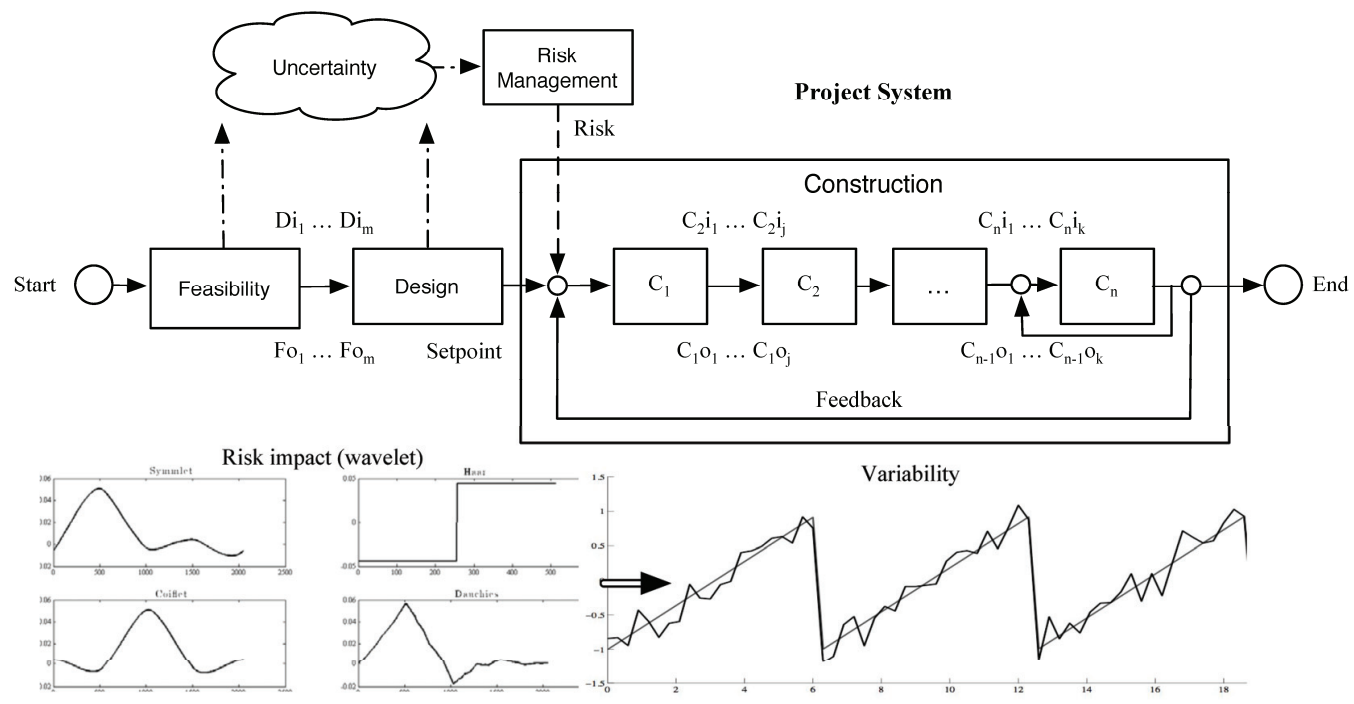

Figure 4. The project construction system from the theoretical framework.

The opportunity for improvement created by process repetitiveness reveals a similarity between the resulting theoretical framework and the concept of the control loop, or feedback loop, which controls the dynamic behaviour of the system. The block diagram shows the flow of signals across a system. The block diagram is common to control systems, where a system output may determine an input for the following system. Alternatively, a posterior system can affect the output of previous systems in the chain [100] as in the case of a closed-loop system. Possible categories of inputs on a construction system are tools, equipment, labour, management, time, conditions, product, e.g., a meter of the floor installed, or material, e.g., concrete mix, which may constitute the system output [13]. As an illustration, a manager in charge takes corrective action when an activity deviates from tolerances defined in the plan, i.e., management by exception [34]. The control loop in the process control works in an identical manner. Once a set-point (equivalent to project plan) is defined, three tasks occur: measurement (how the activity is going), comparison (whether the activity is performing within the plan limits) and adjustment (corrective actions). If other feedback 
or an inner controller are added to the activity $\mathrm{C}_{n}$ (characterized by the interaction of the operational manager, for example), the system will be structured by two closely linked processes that encapsulate feedback, which constitutes a cascade-control system.

\section{Conclusions}

This literature review conducted an exploration of elements in the building construction project cycle, which possibly influence the project performance, mostly affecting production behaviour. Furthermore, connections and comparisons were made between projects and manufacturing, aimed at identifying key components that could serve as pivotal to the application of successfully proven theories and methods from manufacturing in construction projects. The outcome is a theoretical framework structured as a system. The system connects project stages - feasibility, design and construction - and activities $\left(\mathrm{C}_{m}\right)$, as well as proposes a flow of uncertainty to risk and the risk impact causing variability. This system structure representing the production provides a means to analyse the effects of interconnected elements in a micro and macro view. Although this literature review was comprehensive, the list of unmentioned factors that affect production in construction is still endless. Further research needs to be conducted to identify and describe the effect of construction project particularities on the construction production system. Additionally, further research needs to be conducted to develop a mathematical explanation of the production system that will supply the building construction industry with ways to measure and predict the performance of a production system. System identification approaches hereafter can be used to understand, model and predict tasks, processes and project performance within the proposed framework. Furthermore, the quantification of variability across the building construction system will enable a comprehensive understanding of the behaviour of repetitive processes in projects at the unsteady and steady state, applicable to any current production model that considers a system view. Moreover, in the future, the development of management practices to handle processes at both state conditions will take place.

\section{Author Contributions}

The study was part of a thesis undertaken by the author for correspondence, Ricardo Antunes. It was supervised by Vicente Gonzalez (principal supervisor).

\section{Conflicts of Interest}

The authors declare no conflict of interest.

\section{References}

1. Levy, S.M. Project Management in Construction; McGraw-Hill: New York, NY, USA, 2012; p. 478.

2. United States Department of Labor. Bureau of Labor Statistics. Available online: http://www.bls.gov/ (accessed on 24 September 2014). 
3. McCray, G.E.; Purvis, R.L. Project management under uncertainty: The impact of heuristics and biases. Proj. Manag. J. 2002, 33, 49-57.

4. Abdelhamid, T.S. The Self-Destruction and Renewal of Lean Construction Theory: A Prediction From Boyd-S Theory. In Proceedings of the 12th Annual Conference of the International Group for Lean Construction, Helsingør, Denmark, 3-5 August 2004.

5. Mills, A. A systematic approach to risk management for construction. Struct. Surv. 2001, 19, 245-252.

6. Lutz, J.D.; Hijazi, A. Planning repetitive construction: Current practice. Constr. Manag. Econ. 1993, 11, 99-110.

7. Koskela, L. Application of the New Production Philosophy to Construction; Stanford University: Stanford, CA, USA, 1992; pp. 1-81.

8. Bertelsen, S. Construction as a Complex System. In Proceedings of the 11th Annual Conference of the International Group for Lean Construction, Blacksburg, VA, USA, 21-24 July 2003.

9. Laufer, A. Simultaneous Management: Managing Projects in a Dynamic Environment; American Management Association: New York, NY, USA, 1997; p. 313.

10. Duenyas, I.; Hopp, W.J. Estimating variance of output from cyclic exponential queueing systems. Queueing Syst. 1990, 7, 337-353.

11. Hopp, W.J.; Spearman, M.L. Factory Physics: Foundations of Manufacturing Management, 2nd ed.; Irwin McGraw-Hill: New York, NY, USA, 1996; p. 726.

12. Kumar, S.A.; Suresh, N. Operation Management; New Age International: New Delhi, India, 2009.

13. Bernold, L.E. Simulation of nonsteady construction processes. J. Constr. Eng. Manag. 1989, $115,163-178$.

14. Koskela, L. An Exploration towards a Production Theory and Its Application to Construction; VTT Technical Research Centre of Finland: Espoo, Finland, 2000.

15. Walsh, K.D.; Sawhney, A.; Bashford, H.H. Production equations for unsteady-state construction processes. J. Constr. Eng. Manag. 2007, 133, 245-261.

16. Ohno, T. O sistema toyota de produção além da produção em larga escala; Bookman: Sao Paolo, Brazil, 1988; p. 139.

17. Womack, J.P.; Jones, D.T.; Roos, D. The Machine that Changed the World; Harper Perennial: New York, NY, USA, 1991; p. 323.

18. Poppendieck, M.; Poppendieck, T. Lean Software Development-An Agile Toolkit; Addison-Wesley: New York, NY, USA, 2003.

19. Ries, E. The Lean Startup: How Today's Entrepreneurs Use Continuous Innovation to Create Radically Successful Businesses; Crown Business: New York, NY, USA, 2011.

20. Hopp, W.J.; Spearman, M.L.; Woodruff, D.L. Practical strategies for lead time reduction. Manuf. Rev. 1990, 3, 1-15.

21. Hopp, W.J.; Wu, S.-C. Machine maintenance with multiple maintenance actions. IIE Trans. 1990, 22, 226-233. 
22. Spearman, M.L.; Woodruff, D.L.; Hopp, W.J. CONWIP-A pull alternative to Kanban. Int. J. Prod. Res. 1990, 28, 879-894.

23. Simon, J.T.; Hopp, W.J. Availability and average inventory of balanced assembly-like flow systems. IIE Trans. 1991, 23, 161-168.

24. Koskela, L. In Proceedings of the Lean Construction, National Construction and Management Conference, Sydney, Australia, 17-18 February 1994; Wakefield, R.R., Carmichael, D.G., Eds.; Institution of Engineers: Sydney, Australia, 1994; pp. 205-217.

25. Project Management Institute. Construction Extension to the Pmbok Guide Third Edition, 2nd ed.; Project Management Institute: Newtown Square, PA, USA, 2007; p. 208.

26. Bossink, B.; Brouwers, H. Construction waste: Quantification and source evaluation. J. Constr. Eng. Manag. 1996, 122, 55-60.

27. Hoffman, T.L. Construction management: Added value or added cost? J. Prop. Manag. 1998, 63, 6531300 .

28. Baily, M.N.; Gordon, R.J.; Nordhaus, W.D.; Romer, D. The productivity slowdown, measurement issues, and the explosion of computer power. Brook. Papers Econ. Act. 1988, $19,347-431$.

29. Hossain, M.A.; Chua, D.K.H. Overlapping design and construction activities and an optimization approach to minimize rework. Int. J. Proj. Manag. 2014, 32, 983-994.

30. Li, R.Y.M.; Poon, S.W. Construction Safety; Springer: Berlin/Heidelberg, Germany, 2013; p. 161.

31. Project Management Institute. A Guide to the Project Management Body of Knowledge (Pmbok Guide), 5th ed.; Project Management Institute: Newtown Square, PA, USA, 2013; p. 616.

32. Project Management Institute. A Guide to the Project Management Body of Knowledge (Pmbok Guide), 1st ed.; Project Management Institute: Newtown Square, PA, USA, 1996; p. 182.

33. International Project Management Association. IPMA Competence Baseline Version 3.0, 3rd ed.; International Project Management Association: Nijkerk, The Netherlands, 2006; p. 212.

34. Office of Government Commerce. Managing Successful Projects with Prince2, 5th ed.; The Stationery Office: London, UK, 2009; p. 346.

35. Cho, K.; Hong, T.; Hyun, C. Scheduling model for repetitive construction processes for high-rise buildings. Can. J. Civil Eng. 2010, 38, 36-48.

36. Bhoyar, S.; Parbat, D.K. Optimal scheduling for repetitive construction projects with multiple resource crews. Int. J. Emerg. Technol. Adv. Eng. 2014, 4, 302-307.

37. Tommelein, I.; Riley, D.; Howell, G. Parade game: Impact of work flow variability on trade performance. J. Constr. Eng. Manag. 1999, 125, 304-310.

38. Alarcon, L.F.; Ashley, D.B. Playing Games: Evaluating the Impact of Lean Production Strategies on Project Cost and Schedule. In Proceedings of the IGLC-7, Berkeley, CA, USA, 26-28 July 1999; pp. 263-274.

39. Halpin, D.W.; Kueckmann, M. Lean Construction and Simulation. In Proceedings of the 2002 Winter Simulation Conference, San Diego, CA, USA, 8-11 December 2002; pp. 1697-1703. 
40. Farag, M.A.M. A bridge between increasing reliability and reducing variability in construction work flow: A fuzzy-based sizing buffer model. J. Adv. Manag. Sci. 2014, 2, 287-294.

41. Benjaoran, V.; Dawood, N. Intelligence approach to production planning system for bespoke precast concrete products. Autom. Constr. 2006, 15, 737-745.

42. Thomas, H.R.; Maloney, W.F.; Horner, R.M.W.; Smith, G.R.; Handa, V.K.; Sanders, S.R. Modeling construction labor productivity. J. Constr. Eng. Manag.1990, 116, 705-726.

43. Handa, M.V.K.; Barcia, R.M. Linear scheduling using optimal control theory. J. Constr. Eng. Manag. 1986, 112, 387-393.

44. Bashford, H.H.; Walsh, K.D.; Sawhney, A. Production system loading-cycle time relationship in residential construction. J. Constr. Eng. Manag. 2005, 131, 15-22.

45. Gonzalez, V.; Alarcon, L.F.; Yiu, T.W. Integrated methodology to design and manage work-in-process buffers in repetitive building projects. J. Oper. Res. Soc. 2013, 64, 1182-1193.

46. Gonzalez, V.; Alarcon, L.F.; Molenaar, K. Multiobjective design of work-in-process buffer for scheduling repetitive building projects. Autom. Constr. 2009, 18, 95-108.

47. American Society for Testing and Materials. Building Economics. Available online: http://www.astm.org (accessed on 27 October 2014).

48. Smith, N.J.; Merna, T.; Jobling, P. Managing Risk in Construction Projects, 2nd ed.; Blackwell Publishing: Malden, MA, USA, 2006; p. 257.

49. Association for Project Management. APM Body of Knowledge, 5th ed.; Association for Project Management: Buckinghamshire, UK, 2006; p. 197.

50. Anderson, D.K.; Merna, T. Project management strategy_Project management represented as a process based set of management domains and the consequences for project management strategy. Int. J. Proj. Manag. 2003, 21, 387-393.

51. Project Management Institute. A Guide to the Project Management Body of Knowledge (Pmbok Guide), 3rd ed.; Project Management Institute: Newtown Square, PA, USA, 2004; p. 405.

52. Gibb, A.; Isack, F. Re-engineering through pre-assembly: Client expectations and drivers. Build. Res. Inf. 2003, 31, 146-160.

53. Blismas, N.; Pasquire, C.; Gibb, A. Benefit evaluation for off-site production in construction. Constr. Manag. Econ. 2006, 24, 121-130.

54. Borg, R.; Gambatese, J.; Haines, K.; Hendrickson, C.; Hinze, J.; Horvath, A.; Koehn, E.; Moritz, S.; Mass, M.; Haughney, R. Rebuilding the world trade center. Pract. Period. Struct. Des. Constr. 2003, 8, 137-145.

55. Wyrik, D.A.; Eschenbach, T.G. Remote oil and gas facility construction. J. Constr. Eng. Manag. 1989, 115, 228-236.

56. Briscoe, G.; Dainty, A.R.J.; Millett, S. Construction supply chain partnerships: Skills, knowledge and attitudinal requirements. Eur. J. Purch. Supply Manag. 2001, 7, 243-255.

57. Said, H.; El-Rayes, K. Optimal utilization of interior building spaces for material procurement and storage in congested construction sites. Autom. Constr. 2013, 31, 292-306.

58. Baye, M.R. Magerial Economics and Business Strategy, 7th ed.; McGraw-Hill: New York, NY, USA, 2010; p. 658. 
59. McGuigan, J.R.; Moyer, R.C.; Harris, F.H.D. Managerial Economics: Applications, Strategy, and Tactics, 12th ed.; South-Western: Mason, OH, USA, 2011; p. 792.

60. AACE International. Risk Analysis and Contingency Determination Using Expected Value. In TCM Framework: 7.6 - Risk Management; AACE International: Morgantown, WV, USA, 2012; Volume 44R-08, pp. 1-14.

61. Project Management Institute. Managing Change in Organization: A Practice Guide; Project Management Institute: Newtown Square, PA, USA, 2013; p. 141.

62. Sutherland, J. Scrum Log Jeff Sutherland: Why Gantt Charts Were Banned in the First Scrum. Available online: http://scrum.jeffsutherland.com/2006/02/why-gantt-charts-werebanned-in-first.html (accessed on 14 April 2014).

63. Deemer, P.; Benefield, G.; Larman, C.; Vodde, B. The Scrum Primer. In A Lightweight Guide to the Theory and Practice of Scrum; Available online: http:/assets.scrumfoundation.com/ downloads/1/scrumprimer20.pdf?1352449266 (accessed on 2 July 2014).

64. Mandal, A.K. Introduction to Control Engineering Modeling, Analysis and Design; New Age International Publishers: New Delhi, India, 2006; p. 633.

65. Weiss, S.I. Product and Systems Development a Value Approach; John Wiley \& Sons: Hoboken, NJ, USA, 2013; p. 275.

66. Vidal, L.-A.; Marle, F.; Bocquet, J.-C. Modelling project Complexity. In Proceedings of the International Conference on Engineering Design, ICED’07, Paris, France, 28-31 July 2007; pp. $1-10$.

67. Baccarini, D. The concept of project complexity a review. Int. J. Proj. Manag. 1996, 14, 201-204.

68. Mitchell, M. Complexity a Guided Tour; Oxford University Press: Oxford, UK, 2009; p. 366.

69. Curlee, W.; Gordon, R.L. Complexity Theory and Project Management; John Wiley \& Sons: Hoboken, NJ, USA, 2011; p. 419.

70. Project Management Institute. Practice Standard for Work Breakdown Structures, 2nd ed.; Project Management Institute: Newtown Square, PA, USA, 2006; p. 123.

71. Project Management Institute. Practice Standard for Work Breakdown Structures, 2nd ed.; Project Management Institute: Newtown Square, PA, USA, 2006; p 156.

72. Kezner, H. Strategic Planning for Project Management Using a Project Management Maturity Model; John Wiley \& Sons: New York, NY, USA, 2001; p. 271.

73. Kaimann, R.A. Coefficient of network complexity. Manag. Sci. 1974, 21, 172-177.

74. Vidal, L.-A.; Marle, F.; Bocquet, J.-C. Measuring project complexity using the analytic hierarchy process. Int. J. Proj. Manag. 2010, 29, 718-727.

75. Project Management Institute. Navigating Complexity: A Practice Guide; Project Management Institute: Newtown Square, PA, USA, 2014; p. 114.

76. Temperley, H.M.V. Graph Theory and Applications; Halsted Press: New York, NY, USA, $1981 ;$ p. 130.

77. Nassar, K.M.; Hegab, M.Y. Developing a complexity measure for project schedules. J. Constr. Eng. Manag. 2006, 132, 554-561. 
78. Saaty, T.L. Multicriteria Decision Making: The Analytic Hierarchy Process: Planning, Priority Setting, Resource Allocation, 2nd ed.; RWS Pubns: Pittsburgh, PA, USA, 1990.

79. Mello, P.C.D. John Maynard Keynes, Presidente de Companhia de Seguros; Pedro Carvalho de Mello: São Paulo, Brazil, 2011; pp. 1-152.

80. Keynes, J.M. The General Theory of Employment, Interest, and Money; Harcourt Brace \& World: New York, NY, USA, 1936; p. 403

81. European Commission. Value Management; European Commission: Brussels, Belgium, 1995; p. 190.

82. Perminova, O.; Gustafsson, M.; Wikstrom, K. Defining uncertainty in projects-A new perspective. Int. J. Proj. Manag. 2008, 26, 73-79.

83. Knight, F.H. Risk, Uncertainty and Profit; Library of Economics and Liberty: Boston, MA, USA, 1921; p. 381.

84. Crouhy, M.; Galai, D.; Mark, R. The Essentials of Risk Management; McGraw-Hill: New York, NY, USA, 2005; p. 416.

85. Mun, J. Modeling Risk: Applying Monte Carlo Simulation, Real Options Analysis, Forecasting, and Optimization Techniques; John Wiley \& Sons: Hoboken, NJ, USA, 2006; p. 623.

86. Project Managment Institute. A Guide to the Project Management Body of Knowledge (Pmbok Guide), 4th ed.; Project Management Institute: Newtown Square, PA, USA, 2008; p. 507.

87. Jorion, P. Value at Risk the New Bechmark for Managing Financial Risk, 3rd ed.; McGraw-Hill: New York, NY, USA, 2007; p. 594.

88. Cleden, D. Managing Project Uncertainty; Gower: Surrey, UK, 2009; p. 146.

89. Wikipedia. Variability. Available online: https://en.wikipedia.org/wiki/Variability (accessed on 22 April 2014).

90. Radosavljević, M.; Horner, R.M.W. The evidence of complex variability in construction labour productivity. Constr. Manag. Econ. 2002, 20, 3-12.

91. Atkinson, R.; Crawford, L.; Ward, S. Fundamental uncertainties in projects and the scope of project management. Int. J. Proj. Manag. 2006, 24, 687-698.

92. McConnell, S. Software Estimation: Demystifying the Black Art; Microsoft Press: Redmond, WA, USA, 2006; p. 352.

93. Spencer, M.S.; Cox, J.F. An analysis of the product-process matrix and repetitive manufacturing. Int. J. Prod. Res. 1995, 33, 1275-1294.

94. Public Broadcasting Service (PBS). One World Trade Center. In Super Skyscrapers; PBS: Arlington, VA, USA, 2014.

95. Shaked, D. Strength-Based Lean Six Sigma: Building Positive and Engaging Business Improvement; Kogan Page Ltd.: London, GBR, 2013.

96. Taghizadegan, S. Essentials of Lean Six Sigma; Elsevier: Amsterdam, NL, USA, 2006; p. 275.

97. University of Alberta Dictionary. Universal Function Approximator. Available online: http://www.bcp.psych.ualberta.ca/ mike/Pearl_Street/Dictionary/contents/U/universalfn.html (accessed on 5 July 2014).

98. Xu, J.-X.; Tan, Y. Linear and Nonlinear Iterative Learning Control; Springer: Berlin, Germany, 2003; Volume 291, p. 177. 
99. Dager, R.; Zuazua, E. Wave Propagation, Observation and Control in 1-d Flexible Multi-Structures; Springer: New York, NY, USA, 2006; Volume 50, p. 227.

100. Ogata, K. Modern Control Engineering, 5th ed.; Prentice Hall: Upper Saddle River, NJ, USA, 2010 ; p. 905. 

Part III:

Urban Issues 



\title{
Understanding the Importance of Urban Amenities: A Case Study from Auckland
}

\section{Natalie Allen}

\begin{abstract}
Along with many Pacific Rim cities in Australia and North America, Auckland, New Zealand has enacted an urban growth management strategy premised on two concepts: "liveability" and a "quality compact city". The effective implementation of this strategy will, in part, require higher density housing typologies to be developed within the existing suburban fabric. The urban amenities in a neighbourhood play an important role in providing a sense of liveability for residents. This paper examines these issues by evaluating and reporting on key outcomes from 57 face-to-face qualitative interviews with residents who currently live in medium density housing in four Auckland suburbs; Takapuna, Kingsland, Botany Downs, and Te Atatu Peninsula. Findings consider the trade-offs residents make when choosing to live in medium density housing typologies, how they value the urban amenities in their neighbourhood and the role they think these amenities play in their location satisfaction. Conclusions are drawn around how the resident-derived information may inform the market on the supply side of housing, and comment is made about how these preferences may, or may not, respond to the objectives of the underlying urban management strategies involved.
\end{abstract}

Reprinted from Buildings. Cite as: Allen, N. Understanding the Importance of Urban Amenities: A Case Study from Auckland. Buildings 2015, 5, 85-99.

\section{Introduction}

This research responds to the urban intensification debate in Auckland by questioning the perceived role of urban amenities in promoting quality of urban life at higher densities in four of the city's suburban neighbourhoods: Takapuna, Kingsland, Albany, and Botany.

Like Auckland, many Pacific-Rim cities in Australia and North America have long imbued compact city principles in to their urban growth management strategies. Cities such as Brisbane, Sydney, Melbourne, Vancouver, and Portland are facing a similar set of development issues related to their growing yet ageing populations and shifting demographics towards smaller households. While a system of common law is in place, governmental and institutional arrangements do differ between the cities [1]. Unlike Auckland, for example, each of the aforementioned cities has both national, state, and local (city-wide) levels of planning governance, whereas Auckland has national and local only. This changes funding streams and methods for implementation. Despite these structural differences, notable similarities exist between the growth management strategies of these cities including their greenbelt ideology that utilises an urban growth boundary to restrict greenfield development at urban peripheries [2] as well as the promotion of networks of higher density mixed-use development clustered around walkable town centres.

There have been various waves of higher density housing trends, across many Pacific-Rim cities in Australasia and North America, including Auckland [3,4]. However, the quality compact goals of these cities largely exist in contradiction to the dominant post-war ethos of suburbia still prevalent 
despite the considerable regeneration and redevelopment of their downtowns, waterfronts and other former-industrial land. Resident resistance to suburban intensification across these Pacific-Rim cities has been widely noted [5,6]. Writing about traditional aspirational housing ideals both Alves [3] and Randolph [4] in Australia, and Smith and Billig in North America, identify a long-standing preference for suburban single-storey detached dwellings. This trend is referred to as the Quarter-Acre Pavlova Paradise in New Zealand [7]. Alves identifies that research and planning action has been lacking in suburban areas [3]; transforming and intensifying suburbia, while still maintaining the distinct character and perceived liveability of the suburban model, is the next big challenge for urban planners and designers.

In this research it is argued that a key element in the transition to more urbanised environments is related to the extent to which urban amenities have a role in resident perceptions of quality of urban life. Mulligan and Carruthers identify that "amenities are key to understanding quality of life because they are precisely what make some places attractive for living and working, especially relative to other places that do not have them and/or are burdened with their opposites, disamenities" [8] (p. 107).

Urban amenities are understood in this research to mean specific urban facilities that contribute to the urban living experience of residents [9]; they are linked to the daily life needs of residents in a neighbourhood. Some examples given by Randall include: "grocers, convenience stores, access to public transit, schools and professional services [doctor or dentist]" [10] (p. 47). Gottlieb confirms that "residential amenities may be defined as place-specific goods or services that enter the utility functions of residents directly" [11] (p. 1413). Both Mathur and Stein [12] (p. 252) and McNulty et al. [13] refer to urban amenities as "quality of life factors" and Howie et al., confirm that "urban amenities are generally accepted as being important to a household's sense of place" [14] (p. 235). There are both public sector amenities provided by councils, such as parks, public squares and recreational facilities, as well as private sector amenities such as cafés, restaurants, retail and other goods or service providers.

There are two main reasons given in the literature as to why focusing on the role of urban amenities in the delivery of urban intensification is important. Firstly, in an economic sense, it is argued that a diversity of urban amenities attract economic activity to a city in terms of firms and labour wanting to be located in a place of high amenity value [8,15-17]. In other words, "the provision of amenities generates urban advantages that perpetuate the concentration of economic activity and population in, and in closer proximity to, them" [18] (p. 40). Mathur and Stein also confirm that "the emerging literature on amenities seems to indicate that one of the most effective ways to attract knowledge workers in the regions and promote economic development is the creation of amenities" [12] (p. 265).

In line with economic reasoning, the second broader reasoning is that the accessibility and convenience of urban amenities contribute to quality of urban life experiences [19-22]. As society changes and evolves so too do people's quality of life requirements and aspirations. There are clear linkages acknowledged in the literature between the provision of varied urban amenities and changing lifestyles and aspirations [1,23,24]; demographic changes for example have a direct impact on the spatial configuration of the city and are closely tied to changing lifestyle preferences. 
Increasing ethnic diversity through globalisation also contributes to urbanism trends as new city residents bring their own understandings of intensification and the relationship between urban amenities and perceived quality of life. There are also issues of affordability and potential new home owners now being priced out of the market; these buyers may turn to higher densities as a way of entering the property market. Internationally, authors such as Clark [25] have recognised that demographic and ethnographic changes alter the way cities are viewed and experienced and as such, the provision of urban amenities and their integration in to urban areas must also be reconsidered. Randolph [4] in particular, writing about Australia, highlights the need for planners to understand the amenity requirements for higher density neighbourhoods, particularly if more children are going to be living in these urbanised environments, thus increasing the need for schools, child care facilities, and recreational areas. An example given by Schmitz et al. is that the increasing number of "work-at-homers" "often feel isolated in typical suburban communities and would like access to the amenities that are available to downtown office workers" [26] (p. 6). They consider that options such as the corner coffee shop, lunch bars, a print centre, local gym or recreation area, and retail facilities should be better integrated in to suburban environments. Auckland Council also acknowledges that the city needs "more housing adjacent to local shops and services, public open spaces and areas with expansive views" [27] (p. 70). And yet, research into how this might occur and how different amenities are valued by residents is very limited in New Zealand. In this paper, understanding the relationship between urban amenities and perceived quality of life is based on the premise that "dwellings are important, but so too is the location of the dwelling" [28] (p. 98).

Whatever the reasons for intensification, the question remains: if suburbia transforms, will these higher density neighbourhoods meet the aspirations and needs of future residents? It is therefore necessary to ask residents about the urban amenities they use and value and the relationships they see between these amenities and their sense of location satisfaction.

\section{Method}

Guided by a constructivist Grounded Theory approach the researcher conducted 57 hour-long face-to-face structured interviews with local residents from four case study neighbourhoods in Auckland: Takapuna, Kingsland, Botany Downs, and Te Atatu Peninsula (see Figure 1). The structured interviews were recorded and transcribed. The analysis included initial line-by-line manual coding before open and selective coding phases were conducted by the researcher in NVivo. Theoretical coding was the third coding phase in line with Grounded Theory methods.

The chosen neighbourhoods represent both inner and outer fringe belt suburbs [29] that have all experienced strong increases in both rental and owner-occupier medium density developments. They range from being 4.3 to $20 \mathrm{~km}$ away from Auckland's Central Business District (CBD) and are located to the north, west, and south-east of Auckland. Within these neighbourhoods, medium density case study developments were chosen as the locations where direct mailbox dropping would occur to attract the interviewees for the study. 


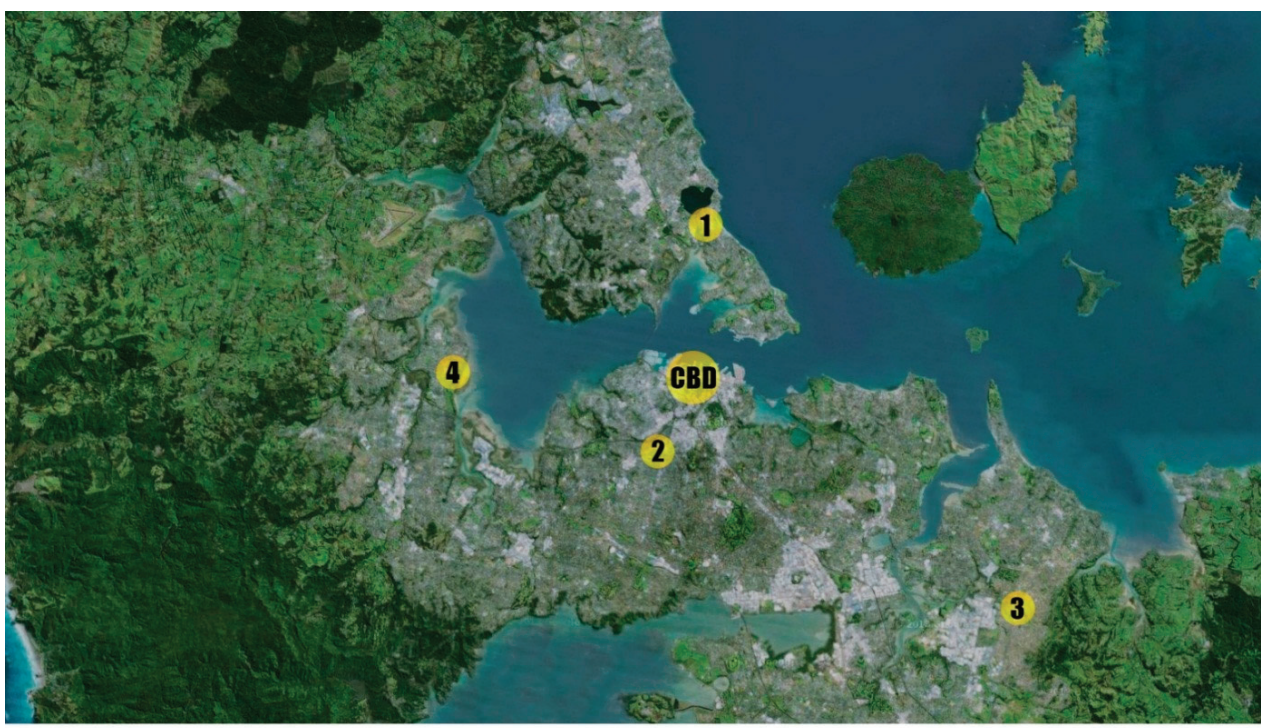

$2 \mathrm{kms}$

2 miles
Key

1. Takapuna

2. Kingsland

3. Botany Downs

4. Te Atatu Peninsula

Figure 1. The four case study neighbourhoods (Source Google Maps).

The criteria for selecting the developments within the case study neighbourhoods included: that each of the developments was multi-unit, at a Net Residential Density (NRD) greater than 35 dpHA, and in a location that provided local amenities; for example a town centre. This meant a mix of typologies ranging from three- and five-storey apartment complexes to attached townhouses and units. Developments also had to have been established in their communities for a period of more than three years and accessible for interviews. The developments at 130 Anzac St. in Takapuna, 435 New North Road in Kingsland, and 84 Gunner Drive in Te Atatu required approval from building managers to mail box drop as their mail boxes were concealed within the developments.

\subsection{Takapuna}

Takapuna is a city fringe suburb in the north of Auckland, $9.6 \mathrm{~km}$ from the CBD. It has been extensively considered for intensification by North Shore City Council and Auckland Regional Council. It has also been identified as a key growth area in the Auckland Plan [30] and the Unitary Plan [31]. Takapuna was also identified by Fontein [32] as an area that is well placed for market-led intensification due to its access to a range of amenities; including, transit, employment opportunities, mixed use residential and business land, and natural amenities [33] (pp. 33-35). Twenty residents from three developments in Takapuna were interviewed. These developments ranged from townhouses with a Net Residential Density (NRD) of 35.8 dpHA to three-storey apartments that were $60.8 \mathrm{dpHA}$ to even higher density five-storey apartments that were $167.9 \mathrm{dpHA}$ (see Figure 2 and Table 1). 


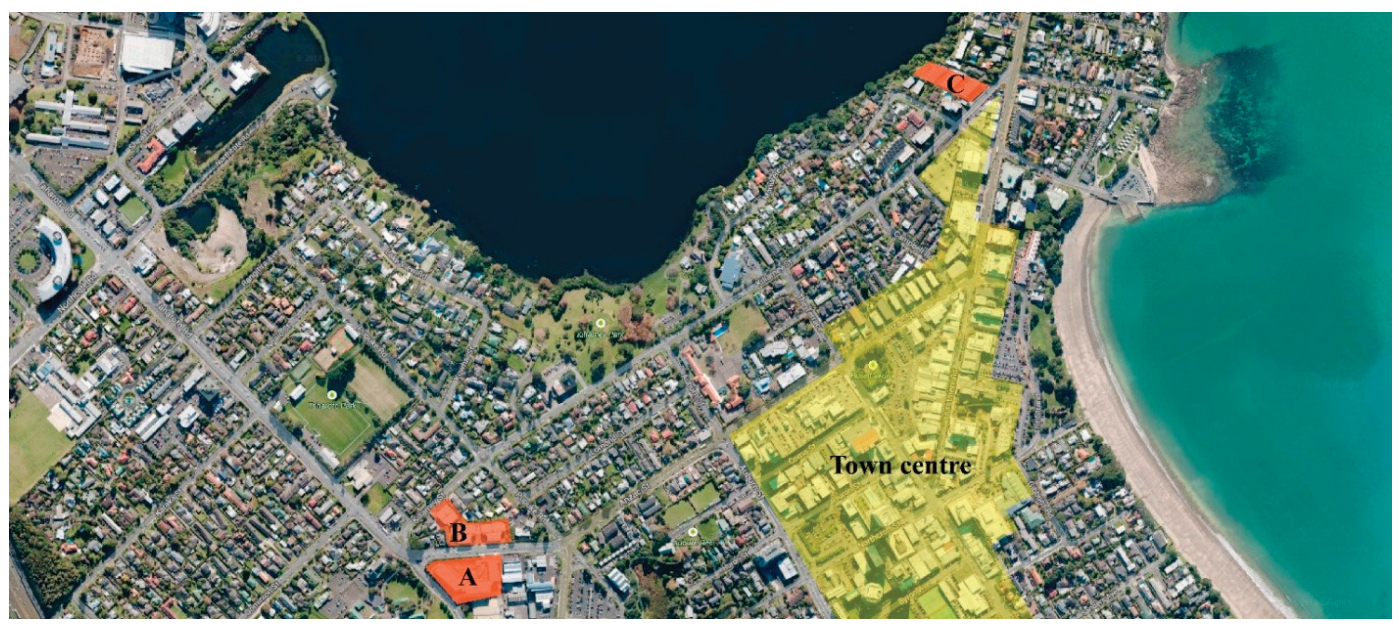

Figure 2. Takapuna case study area: interviewees were from the developments shown in orange, the town centre is shown in yellow. Letters correspond to Table 1. (Source: Google Maps).

\subsection{Kingsland}

Kingsland is a city fringe suburb, just $4.3 \mathrm{~km}$ from the CBD, and one of the top five rental growth suburbs in Auckland [34]. While it has grown in popularity because of its proximity to a range of urban amenities and access to the CBD, it remains predominantly suburban, made up of single-storey detached dwellings, and a noticeably smaller town centre than the other chosen areas. Medium density housing in the area is increasing; although currently these are largely apartments rather than a mix of medium density typologies such as terraced houses and units. Thirteen residents from two developments were interviewed. These developments were both four-storey apartment buildings at densities of 281.3 and 285.7 dpHA (see Figure 3 and Table 1). One was a mixed-use typology with apartments built above the high street shops. In this case it was four storeys on the road side but six storeys on the parallel street where the site contour sloped down. 


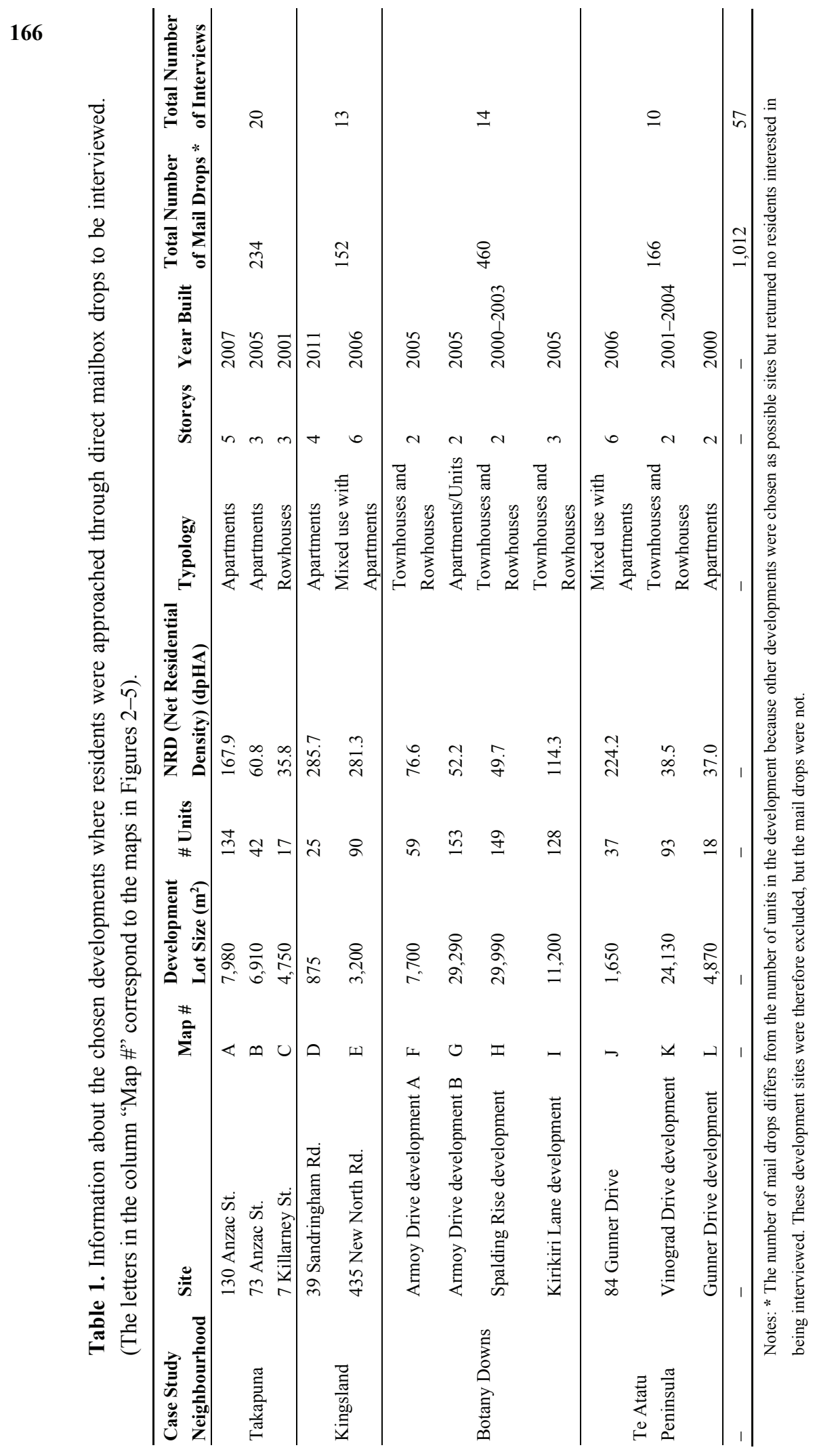




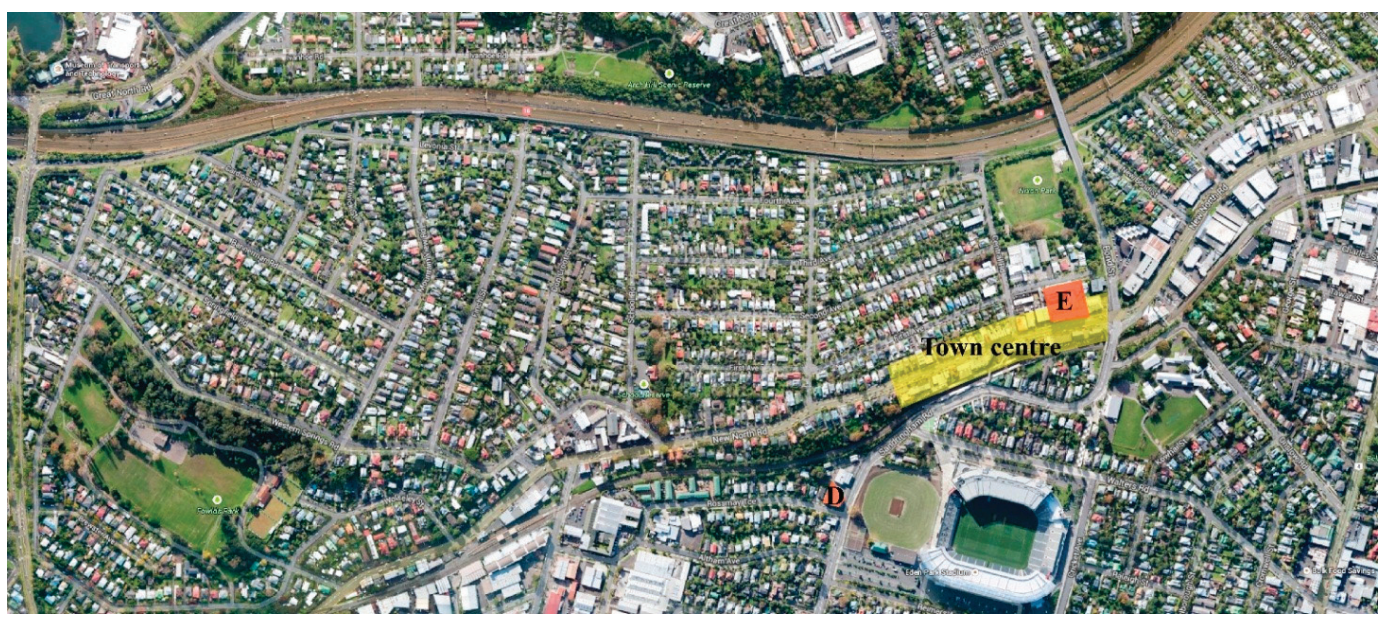

Figure 3. Kingsland case study area: interviewees were from the developments shown in orange, the town centre is shown in yellow. Letters correspond to Table 1. (Source: Google Maps).

\subsection{Botany Downs}

Botany Downs is the furthest suburb, at $20 \mathrm{~km}$ south-east from Auckland's CBD, considered in this research. It is an area that has seen considerable growth in the last 5-10 years, frequently in the form of two-storey attached dwellings in large scale, partially gated communities. These developments ranged from 49.7 to 114.3 dpHA (see Figure 4 and Table 1). 14 residents were interviewed from four developments in Botany Downs.

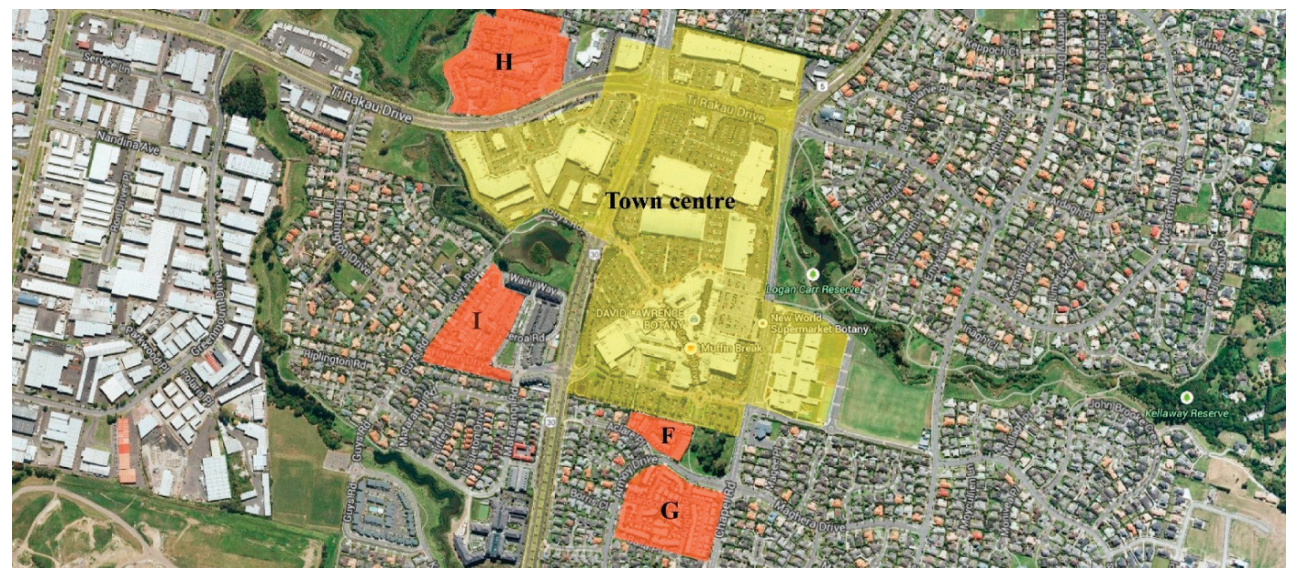

Figure 4. Botany Downs case study area: interviewees were from the developments shown in orange, the town centre is shown in yellow. Letters correspond to Table 1. (Source: Google Maps). 


\subsection{Te Atatu Peninsula}

Te Atatu Peninsula is $15.3 \mathrm{~km}$ to the west of Auckland's CBD. While it is dominated by low density single-storey detached dwellings its popularity and increasing levels of gentrification have seen attached and semi-attached two-storey townhouses develop to the east of the town centre. 10 residents from three development areas were interviewed. Two of the developments range in density from 37 to 38.5 dpHA. However, the third is a six-storey apartment building, with retail on the ground floor, that has been developed on the main street leading in to the town centre. It has a relatively high density of 224.2 dpHA; considerably greater that the surrounding suburban area that measures between 10 and 20 dpHA (see Figure 5 and Table 1).

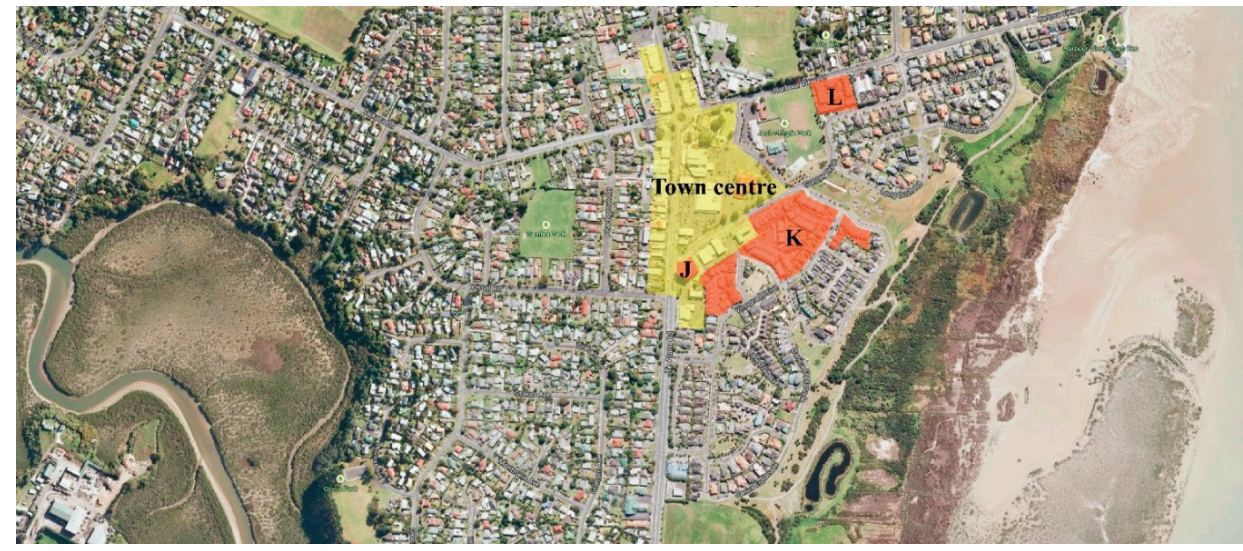

Figure 5. Te Atatu Peninsula case study area: interviewees were from the developments shown in orange, the town centre is shown in yellow. Letters correspond to Table 1. (Source: Google Maps).

Table 1 is a summary of information about the chosen developments across the four case study neighbourhoods including the lot size, number of units, Net Residential Density (NRD), their typology, the number of storeys and the year they were built. It also shows the total number of mail drops conducted and the number of interviews conducted in each area. In total 36 females and 21 males responded to the mail-box letter drops. Interviewees were spread between the ages of 23 and 87; the average age of respondents was 44 . Of the 57 interviewees, 26 were owner-occupiers and 31 were renters. The average length of dwelling tenure was three years; the shortest time being one month and the longest 13 years. Seven interviewees lived alone, 25 were couples, six interviewees lived with flatmates, and two lived with extended family members. Seventeen had a least one child living at home, of these 10 had children under seven. Only three had two or more children living at home.

Thirty-six of the interviewees were born in New Zealand and nearly half of these had also spent time living overseas where they were exposed to a range of different lifestyles and housing norms. Of the 21 born overseas, only 11 were from countries where English was not their first language. Countries of origin included The United States of America, Australia, Scotland, England, China, 
Korea, Malaysia, Vietnam, Singapore, India, Portugal, Bulgaria, Slovakia, Serbia, Jordan, Mozambique, and South Africa. Nineteen of the interviewees had had experience living outside of Auckland in areas ranging from Christchurch and Wellington to rural Otago, Napier, Gisborne, Palmerston North, Tauranga, Rotorua, and Tairua. Despite the geographic, demographic, and socioeconomic variations between the case study suburbs and the interviewees, clear patterns emerged among the findings.

Interview questions covered household structure, dwelling tenure, current employment and travel habits as well as housing histories and aspirations. Interviewees were asked about the urban amenities they use, how often, their accessibility, and how they valued them. How residents defined quality of life and their perceptions of urban intensification and density were also explored.

\section{Results}

\subsection{Making Medium Density Housing Choices}

All the interviewees, excluding two, had had previous experience living in standalone houses; most often it was the housing norm they had experienced the most. Over half, $58 \%$ of the interviewees, had also experienced apartment or townhouse living at some stage prior to their current housing experiences; whether it be as students, while living overseas, or while living in a flatting situation away from their regular family home.

When asked about their process for making housing choices 41 interviewees mentioned proximity to urban amenities and the resultant convenience as one of their main reasons for moving to their current housing. Medium density typologies also played a factor, ease of maintenance in particular was stated as affecting housing choices for 26 of the interviewees. Twelve interviewees mentioned trading-off living in a standalone house for an apartment or townhouse because they found them to be warmer and cleaner than the available standalone housing stock they could rent for a similar price point. Safety, security, proximity to work, affordability, and place attachment created due to proximity to friends and family were other reasons frequently mentioned by interviewees as reasons that had influenced their decision making when deciding to live in their current location.

\subsection{Future Housing Aspirations}

More than half of interviewees saw themselves continuing to live in the same suburb where they currently resided over the coming five years. Where this was not the case the predominant aspirations held were to look at land on the urban fringe for affordability reasons, or to move to a more rural environment as part of long term lifestyle ambitions. Retirees interviewed were split between moving out of Auckland or to fringe areas where they could live quieter lives or staying in apartment typologies, usually in their current neighbourhood, that would be easy for them to maintain or lock up and leave if they planned to travel. Where this was the case proximity to a range of urban amenities and the ability to walk or use public transport were noted as key location drivers to support their active urban lifestyles.

Twenty-two interviewees mentioned their or their partners proximity to work as part of their decision making process around housing and reasoning for wanting to stay in the same area. This 
largely came down to the notion that interviewees did not want to be "sitting in traffic half the day". Place attachment due to family and friends living in the same area was also frequently mentioned as a factor affecting the interviewees' location aspirations.

When asked about the type of housing they saw themselves living in in five years' time 26 interviewees saw themselves in medium density typologies, including low rise apartments (of three to five storeys) or terraced housing (similar to their current typologies). Twenty-five saw themselves in low density typologies including single detached dwellings on small or large sections and six were unsure what their housing aspirations were at the present time. Of the 25 interviewees who identified an aspiration for low density typologies, five of them simultaneously discussed medium density options as being an equally likely aspiration they would be happy with. This would shift the total number of those who aspired to medium density housing solutions from 26 to 31 respondents. Typologies at the same or higher densities than their current housing scenarios, namely low rise apartments and terraced housing, were also the notably favoured "back-up" plan for interviewees when they were asked where they might be happy to live if their first choice was not available to them for whatever reason.

The concept of "lifestyle" was used by interviewees as a reason to justify both their low and medium density preferences. Those who wanted standalone houses as their first choice usually spoke about wanting space, peace and quiet, the ability to have pets and a garden, and "the ability to cater for acquisitions which you can't do in a smaller apartment". A small number of interviewees who aspired to live in a standalone house did acknowledge that many of the issues they currently had with medium density typologies, particularly related to storage and shared garden space, could be addressed if the apartments or terraced houses were well designed, built from quality materials, and larger than the CBD "shoeboxes" that they currently associated with the idea of "higher density living".

Those who wanted to live in low rise apartments or terraced houses cited proximity to urban amenities as their main reason for choosing this typology over others, stating that they "like going out and doing things and having everything on our doorstep". One interviewee commented, "I'll compromise on smallness... I don't actually mind the smallness, but I like to be close to the city". When asked why, the interviewee commented about the liveliness of the city contributing to their quality of life because they were always busy and engaged with what was going on around them.

Other reasoning given for preferring medium density typologies included security and their low maintenance nature. One interviewee commented that they wanted to live "somewhere that is clean, tidy and nice. I really don't want a garden that gets neglected because I don't have the time for it. I'd rather not have it at all'. Another interviewee saw living in an apartment as meaning they could pay off their small mortgage and "make a bit of a nest egg" so that they could travel and enjoy their upcoming retirement.

A number of interviewees who saw themselves living in medium density dwellings over the next 5 years, and in some cases longer term, did temper their aspiration with the concern that many apartments currently available were very small and did not always meet their needs; lack of storage, lack of car parking for multi-bedroom apartments, and small kitchens rather than four burner hobs were the most frequently mentioned concerns. One interviewee spoke of wanting to live in "stacked 
villas", the villa being a popular traditional standalone housing typology in New Zealand. They wanted a villa on the third or fourth floor; in other words a spatial layout that they were familiar with that had larger shared spaces, more storage, and a larger outside living area than the majority of apartments they had seen previously in Auckland.

Additional reasoning given by those interviewees who stated that they would prefer to live in a standalone house related to their desire to renovate and because they thought that owning a home was part of the "kiwi dream". Fear that the build quality of apartments would be substandard was also cited as a concern by some interviewees. A further concern was that they thought buying an apartment would disadvantage them financially because the capital gains were perceived to be higher for properties with standalone houses. One interviewee who currently owned their apartment commented that they would eventually like to buy a house "out in the suburbs" but that it "purely would be an investment property and we'd stay living in the apartment".

\subsection{Views about Quality of Life and Urban Amenities}

When interviewees were asked directly about how they defined quality of life their responses covered a broad range of issues; the importance of health, income, safety, and broader discussions of freedom, enjoyment and fulfillment were raised. Twenty-four interviewees related their perceptions of quality of life to concepts of neighbourhood and the availability and accessibility of urban amenities. When asked about the perceived role of urban amenities in a neighbourhood, all 57 interviewees linked it to quality of life aspects, in particular to notions of accessibility and the associated convenience or ease this bought to their lives.

When asked to define the term urban amenities seven interviewees thought of them predominantly as being council provided amenities. Examples given included public transport, public pools, community centres, and libraries. Four were unsure of the terms specific meaning. The remaining 43 interviewees thought of urban amenities as all the services and infrastructure they used in their daily lives. One interviewee commenting that "urban amenities would be all of the things that you value in a community and so that would be the open space, the walkways, the park space, the variety of shops or facilities and our proximity to them".

Food related amenities, such as supermarkets, cafés, and restaurants, were the urban amenities considered to be the most important by interviewees. Supermarkets were specifically mentioned the most frequently. Recreation amenities and public spaces were also favoured. The areas where residents reported using their local amenities daily and usually accessed them by foot (this was weather dependent) were Takapuna and Botany Downs. Te Atatu Peninsula had similar responses for a variety of food and community amenities but many interviewees commented about their needing to drive for retail amenities. While Kingsland residents were positive about their local café and restaurant culture, walkability for urban amenities such as chemists, clothing stores, dairies and supermarkets was questioned by the interviewees who predominantly drove to neighbouring suburbs for these urban amenities because they were not available to them in Kingsland. 


\section{Discussion}

The initial findings on housing choices in Auckland are in line with the work of Haarhoff and Beattie [35,36], Mead and McGregor [37], and Syme et al. [5] who all found that residents living in medium density developments in Auckland rated highly the convenience of living in close proximity to the urban amenities that they valued in their daily lives; including shops, cafés and restaurants, schools, their workplaces, public transport, and public spaces (particularly if they were park and recreation facilities). These studies also all identified that the locations of these urban amenities and their convenience was a significant factor for residents in choosing to live where they did.

A surprising change from previous studies, where standalone houses featured prominently as the favoured typology residents saw themselves living in, was the relatively even divide between the aspirations of residents being low density versus medium density typologies. The interviews suggest that the increasing popularity of low rise apartments and terraced housing can be tied to changing lifestyle expectations in the city across a broad spectrum of demographics and lifestages. The shift to favouring similar to higher densities than the interviewees were already living in suggests a disjuncture between the current supply and potential demand for higher density typologies in Auckland's suburbs.

However, the concern that current medium density typologies did not meet the daily life needs of residents, raises questions about how some interviewees might have responded to questions about their housing aspirations if more medium density typology options were available to them within their current suburban areas. The interviewee responses would suggest that a greater acceptance of medium density typologies may occur if concerns about build quality and lack of storage and kitchen space were addressed.

Furthermore, when questioned about how they defined urban amenities the overwhelming majority of interviewees thought of them as all the services and infrastructure they used in their daily lives. This contrasts with much of the planning policy and strategy in New Zealand as well as international literature where amenities are often considered in silos; for example as "natural amenities", "entertainment amenities", or public amenities provided by council. It was therefore found in this research that in order to begin understanding the dynamics of liveability in our current suburban neighbourhoods, "how" residents use and value urban amenities seamlessly across their neighbourhood must be investigated. This means to consider all urban amenities simultaneously as they relate to liveability, whether they be public sector amenities provided by councils, such as parks, public squares and recreational facilities, or private sector amenities such as cafés, restaurants, retail and other goods or service providers. The biggest disjuncture between the findings of this research and current planning policy and strategy in Auckland is just how seamlessly interviewees thought about the spatial relationship between their dwellings and the urban amenities they wanted to live in close proximity to. Current residential zoning, for example, is at odds with this very intimate integration of urban amenities in to the suburban fabric and further research is considerably needed around this issue. 


\section{Conclusions}

A better understanding of how residents are making trade-offs between suburban and urban lifestyle options is critical to understanding the relationship between urban amenities and perceived resident quality of life experiences. Schmitz et al. [26] (p. 62) propose that to consider urban amenities is to programme the urban environment to meet residents' needs. By this thinking, it will be the successful integration of urban amenities in to suburban areas that will enable the perceived quality of life experienced by residents to be maintained or even enhanced during the transition to higher density urban environments to accommodate growth rather than the current low density suburban norm. The apparent risk of not considering urban amenities in this way is to misunderstand the nature of contemporary urban life and the effects of changing demographics and household structures on housing choices.

This research has raised questions about the factors that affect how residents make trade-offs between low density and higher density housing choices; and as such it has raised questions about the effect of the current supply on the future demand for housing in Auckland. Most interestingly, it can be concluded that the majority of those interviewed would trade-off standalone living for low-rise apartment or terraced house living in their current neighbourhoods if the medium density options available to them were of a size and spatial layout that were more similar to the traditional New Zealand home and if urban amenities were integrated in to their neighbourhoods in line with the increasing numbers of residents. Higher amenity must go hand-in-hand with higher density if quality of life is to be experienced in tomorrow's urban Auckland.

\section{Acknowledgments}

The author would like to thank all the residents who agreed to be interviewed as part of this research.

\section{Conflicts of Interest}

The authors declare no conflict of interest.

\section{References}

1. Knox, F.; Smith, J. International Trends and Lessons in Growth Management; Auckland Regional Council: Auckland, New Zealand, 2007.

2. Jerram, R.; Kvan, T. Liveable Melbourne; A Paper Prepared for City of Melbourne Future Melbourne Project by the University of Melbourne; The University of Melbourne: Melbourne, Australia, 2008. Available online: http:/www.futuremelbourne.com.au/wiki/pub/FMPlan/ S3Resources/Liveable_Melbourne_FINAL.pdf (accessed on 22 January 2015). 
3. Alves, T. Medium Density Housing in Melbourne: The Management of Sustainable and Democratic Local Communities under Global Pressure for Increased Urban Efficiencies. In Proceedings of the 19th EAROPH World Planning and Housing Congress and National Housing Conference, Melbourne, Australia, 19-22 September 2004.

4. Randolph, B. Delivering the Compact City in Australia: Current Trends and Future Implications; City Futures Research Centre, University of New South Wales: Sydney, Australia, 2006. Available online: http://www.be.unsw.edu.au/sites/default/files/upload/researchpaper6.pdf (accessed on 22 January 2015).

5. Syme, C.; McGregor, V.; Mead, D. Social Implications of Housing Intensification; Auckland Regional Council: Auckland, New Zealand, 2005.

6. Searle, G. Sydney's Urban Consolidation Experience: Power, Politics and Community. Available online: http://www.griffith.edu.au/_data/assets/pdf_file/0018/48600/urp-rp12searle-2007.pdf (accessed on 16 February 2012).

7. Mitchell, A. The Half-gallon Quarter-acre Pavlova Paradise; Christchurch Whitcombe and Tombs: Christchurch, New Zealand, 1972.

8. Mulligan, G.; Carruthers, J. Amenities, Quality of Life, and Regional Development. In Investigating Quality Urban Life, Theory Methods and Empirical Research; Marans, R., Stimson, R., Eds.; Springer: Dordrecht, The Netherlands, 2011; pp. 107-134.

9. Kelly, A. Securing urban amenity, does it coincide with biodiversity conservation at the local government level? Australas. J. Environ. Manag. 2006, 13, 243-253.

10. Randall, T. Preferences of suburban residents in Thunder Bay, Ontario towards neighbourhood intensification and rediversification. Can. J. Urban Res. 2008, 17, 28-56.

11. Gottlieb, P. Residential amenities, firm location and economic development. Urban Stud. 1995, 32, 1413-1436.

12. Mathur, V.; Stein, S. Do amenities matter in attracting knowledge workers for regional economic development? Pap. Reg. Sci. 2005, 84, 251-269.

13. McNulty, R.J.D.; Penne, L. The Economics of Amenity: Community Futures and Quality of Life; Partners for Livable Places: Washington, DC, USA, 1985.

14. Howie, P.; Murphy, S.; Wicks, J. An application of a stated preference method to value urban amenities. Urban Stud. 2010, 47, 235-256.

15. Auckland Council. A New Kind of Compact: Design-Led Part 3; Auckland Council: Auckland, New Zealand, 2011.

16. Hansen, H.; Winther, L. Amenities and Urban and Regional Development: Critique of a New Growth Paradigm. In Proceedings of the Regional Studies Association Annual International Conference, Pécs, Hungary, 24-26 May 2010.

17. Ministry for the Environment. People, Places, Spaces: A Design Guide for Urban New Zealand; Ministry for the Environment: Wellington, New Zealand, 2002.

18. Partridge, M.M.O.; Alasia, A. Canadian cities as regional engines of growth: Agglomeration and amenities. Can. J. Econ. 2007, 40, 39-68. 
19. Johnson Gardner Consulting. An Assessment of the Marginal Impact of Urban Amenities on Residential Pricing; Portland Metro: Portland, 2007. Available online: http://www.reconnectingamerica.org/assets/Uploads/JohnsonGardner-Urban-Living-Infra-ResearchReport.pdf (accessed on 22 January 2015).

20. Ministry for the Environment. Building Competitive Cities: Reform of the Urban and Infrastructure Planning System; Ministry for the Environment: Wellington, New Zealand, 2010.

21. Rappaport, J. Consumption amenities and city population density. Reg. Sci. Urban Econ. 2008, 38, 533-552.

22. Wheeler, S. Planning for SustaInability; Routledge: New York, NY, USA, 2004.

23. North Shore City Council. Good Solutions Guide for Mixed Use Development in Town Centres; North Shore City Council: Auckland, New Zealand, 2005.

24. Parliamentary Commissioner for the Environment. The Cities and Their People: New Zealand's Urban Environment; Parliamentary Commissioner for the Environment: Wellington, New Zealand, 1998.

25. Clark, T.; Lloyd, R.; Wong, K.K.; Jain, P. Amenities drive urban growth. J. Urban Aff. 2002, 24, 493-515.

26. Schmitz, A. New Shape of Suburbia: Trends in Residential Development; Urban Land Institute: Washington, DC, USA, 2003.

27. Auckland Council. Technical Report: Centres and Corridors Workstream; Auckland Council: Auckland, New Zealand, 2011.

28. Beacon Pathway Ltd. The Determinants of Tenure and Location Choices of 20-40 Year Old Households in the Auckland Region; The Centre for Housing Research New Zealand: Wellington, New Zealand, 2010.

29. Gu, K. Exploring the fringe belt concept in Auckland, an urban morphological idea and planning practice. N. Z. Geogr. 2010, 66, 44-60.

30. Auckland Council. Auckland Plan; Auckland Council: Auckland, New Zealand, 2012.

31. Auckland Council. Draft Unitary Plan; Auckland Council: Auckland, New Zealand, 2013.

32. Fontein, P.; Ray, A.; Robinson, T. Auckland Plan, Total Auckland Development Potential, Final Report; Auckland Council: Auckland, New Zealand, 2011.

33. Auckland Council. A New Kind of Compact: Design-Led Part 1; Auckland Council: Auckland, New Zealand, 2011.

34. Realestate Investar. 50 Fastest Growth Suburbs in New Zealand. Available online: http: |lwww.realestateinvestar.co.nz (accessed on 4 April 2012).

35. Haarhoff, E.; Beattie, L. Trading Suburbia for an Urban Lifestyle: Obstacles and Opportunities in Auckland, New Zealand. In Proceedings of the 43rd Conference of the Urban Affairs Association, San Francisco, CA, USA, 2-5 April 2013.

36. Haarhoff, E.; Beattie, L.; Dixon, J.; Dupuis, A.; Lysnar, P.; Murphy, L. Future Intensive: Insights for Auckland's Housing; The University of Auckland: Auckland, New Zealand, 2012.

37. Mead, D.; McGregor, A. Intensive Housing Demand and Supply Issues; Auckland Regional Council: Auckland, New Zealand, 2007. 


\title{
Aiming for a Better Public Realm: Gauging the Effectiveness of Design Control Methods in Wellington, New Zealand
}

\section{Morten Gjerde and Brenda Vale}

\begin{abstract}
Design review aims to improve the quality of urban settings, principally by seeking to influence the design of individual buildings positively during the planning approval stages of development. Design review systems were first set up in New Zealand in the mid-1990s in Wellington. The aims of design review are laudable; even if the process is not set up to secure the best possible design outcome it should be able to prevent the worst outrages, so as to ensure a minimal visual quality of streetscapes. However, does design review really achieve what it sets out to do? After a brief summary of design review practices, this article considers whether current design control practices in Wellington are helping to foster well-liked urban streetscapes. Using the results from a recent case study, comparing the aesthetic preferences of the public to those of professionals who participate in design control, the article notes where preferences overlap and where they are different. In the process, the characteristics of buildings and streetscapes that are considered positive and negative are identified. The article goes on to speculate how design review could be made to work more effectively in Wellington.
\end{abstract}

Reprinted from Buildings. Cite as: Gjerde, M.; Vale, B. Aiming for a Better Public Realm: Gauging the Effectiveness of Design Control Methods in Wellington, New Zealand. Buildings 2015, 5, 69-84.

\section{Introduction}

It is never difficult to find opinions critical of the appearance of buildings and cites. When discussing Sydney a decade ago, John Punter made the observation that the city had succumbed to "spectacularly ordinary commercial development" in the period after World War II and that this poor aesthetic quality is only excused by an otherwise discerning public because of Sydney's spectacular setting [1] (p. 406). Making a more general observation, Bentley [2] suggested that the public are united in their dislike of the ways cities have been transformed in recent times. When discussing New Zealand architecture, Layla Dawson of the Architectural Review recently expressed surprise that Auckland has the appearance of an unplanned jumble of high-rise blocks. Why, she asked, would the public put up with such poor architecture [3]? Criticism of New Zealand urban design is even older than this. In 1958 when the architectural historian Nicholas Pevsner visited New Zealand he was hardly complimentary, suggesting there was no town planning in New Zealand, and certainly none in evidence in the central areas of the nation's cities. Even if there were, he wrote, it would be viewed by the non-designer public with suspicion [4]. In a 1958 radio broadcast, Pevsner directed his critique specifically toward Auckland, noting that the city's grid layout was run through the centre with scant regard for the slope toward the Waitamata Harbour or, more generally, for the undulating topography [5]. However, these are the opinions of design specialists not the general public. For the latter does the appearance of the built environment really matter? 
The appearance of the built environment can have a tangible influence over property values, directly affecting the financial well-being of those who own property or conduct a business in an area. The choices people make every day usually include an aesthetic dimension. These may include deciding which city to live in, which house to buy or even which street to walk along [6] (p. 164). It seems that visually attractive places are more sought after by the public, which serves to increase demand and therefore economic values. Increasingly, the built environment is being used as a tool in battles fought between cities for business investment, tourism and high-calibre workers [7,8]. People regularly make choices around matters of aesthetics and it is clear that the visual attractiveness of cities affects the financial well-being of individuals as well as the wider community.

Like many other cities, Wellington, New Zealand has adopted an explicit design control regime as part of its regulatory planning system. Through its District Plan the Wellington City Council seeks to "encourage positive growth that promotes the City's comparative advantages" and lists eight principles to guide development [9]. One of these principles acknowledges that the quality of the public realm is influenced by the buildings and structures that define it. The primary tool used by Council to achieve a high quality public realm is the Central Area Urban Design Guide, the aim of which is to ensure that new developments are coherently designed, make a considered response to their setting and establish positive visual effects [10].

\section{What Is Design Control and How Is It Managed?}

Design control is generally administered as part of the wider regulatory planning system. Regulation of private development emerged in the wake of the Industrial Revolution as it became clear that the free market could not be relied on to deliver appropriate results in a consistent manner. Many urban environments had become unhealthy as well as unattractive through development that had been undertaken to support rapidly expanding industrial activities [11] (p. 25). The bases of contemporary planning regulation can be traced to efforts made in the wake of the Industrial Revolution to ensure that the interests of society as a whole are served when changes to the urban environment are proposed. Under the umbrella of planning regulation, design control is undertaken by local government on behalf of those who must live with the consequences but do not otherwise have a say in the design of new buildings $[12,13]$. Depending on the planning context, regulation is nothing more than a backstop to help ensure that individual projects are built in a manner that does not create untoward environmental effects. Coupled with reliance on the private sector to initiate changes, this means that planning regulation tends to be reactive, rather than proactive, when it comes to managing the built environment.

A number of methods for regulating and controlling the aesthetic outcomes of urban development have been developed and successfully operate in different parts of the world. Punter [14] advises that design review practices can be either regulatory or discretionary. Regulatory (also referred to as administrative) systems are an add-on to zoning controls, where design outcomes are controlled by rules and objective measures. Examples of this are maximum height limits, prescriptions for location of buildings in relation to boundaries, and floor area ratios. These systems provide high levels of certainty to all parties and regulation can be conducted largely through administrative functions, reducing demand on local authority resources. However, they are also criticised for being coarse in 
nature with a tendency toward monotonous environments as there is a tendency for new projects within an area to be built to the same prescribed limits $[6,12]$.

Discretionary systems are more "pragmatic", as they allow regulation to refer to the immediate setting and other variable factors that cannot be accounted for in most rules based regimes. The more successful discretionary systems, in terms of meeting the needs of different stakeholders, are informed by design guidelines or briefs that provide both a target for designers and a reference for those assessing the proposal [15]. While discretionary systems often allow local conditions to be taken into account they are often criticised by the development industry and designers for being arbitrary [16].

Over the past 30 years regulatory design control has tended to become more discretionary, creating scope for development design quality to be scrutinised more closely. Likewise, design guidance informing discretionary systems has become more prescriptive in response to calls for more certainty around the process for applicants. In short, design control systems appear to be converging as they are modified to include both discretionary and regulatory characteristics [14].

The literature outlines a number of challenges for design review. Scheer [16] notes that design review is time consuming and expensive, that it is easy to manipulate through persuasion and pretty pictures, and that it is administered by overworked and inexperienced staff. However, she also suggests that these matters are easy to solve, mainly with additional financial or human resources, through education and by ensuring the process is adequately audited for political involvement. Presenting more substantial challenges for design review are issues relating to power, freedom, justice and aesthetics. These issues exist as tensions between competing social, political or legal forces. The power issue raises questions around who makes the key decisions. Arguing from the perspective of a design professional, Scheer makes the observation that design review is the only field in which lay people (or those not specifically trained in design) can be left to rule over professionals in their areas of expertise.

Freedom of expression in the built environment is another matter that troubles design review. Concerns about freedom are triggered by societal values aligned with property ownership, particularly in the "new world" countries. This concern is most pronounced in the United States although the courts there have consistently upheld the rights of government to control design outcomes [17]. Concerns about justice arise when the reviewer is seen to hold the power, which may limit the extent to which a "fair hearing" is possible. This issue is minimised where written guidelines underpin the planning process. Finally, the murky area of aesthetic preference questions whose values should be referenced. Scheer notes that in many cases guidance remains unhelpfully abstract and projects tend toward soft solutions and mimicry in order to navigate the review process.

Traversing the legal issues raised by design review, George and Campbell [18] identify the various interests that are active in it. Their work addresses the subject in an American context, so freedom of expression is a consistent theme. They highlight the need for variety in the built environment from a physiological perspective. As human wellbeing is affected by the environment, the case for regulation suggests that new designs should avoid inducing unnecessary stress, anxiety or fear in people. However, people also learn and develop their senses through environmental experience and so it can be useful for environments to be challenging. More complex settings, which arise through 
unfettered design decisions, can create such opportunities. It is on this basis that George and Campbell challenge control methods that require new developments to conform to the patterns and design expressions already present in the setting. In addition, pushing the current boundaries of design creates opportunities for development of new theories, new methods and general advancement of the field, which they argue would have wider social benefits. To acknowledge and balance the various interests properly, George and Campbell [18] (pp. 171-173) describe four criteria that a sound design review process must address:

1. A clearly articulated and demonstrable public interest;

2. Demonstrable links to the stated intent;

3. Application early in the design or decision process;

4. Encouraging a variety of acceptable decisions.

Table 1. Principles for progressive design review. Source: Punter [14].

\begin{tabular}{lrl}
\hline \multicolumn{1}{c}{ Heading } & & \multicolumn{1}{c}{ Principle } \\
\hline Community Vision & 1. & $\begin{array}{l}\text { Committing to a comprehensive and coordinated vision of } \\
\text { environmental beauty and design; } \\
\text { Developing and monitoring an urban design plan with community } \\
\text { and development industry support and periodic review; }\end{array}$ \\
\hline Design Planning & 3. & $\begin{array}{l}\text { Harnessing the broadest range of actors and instruments (tax } \\
\text { subsidies, land acquisition) to promote better design; }\end{array}$ \\
and Zoning & 4. & $\begin{array}{l}\text { Mitigating the exclusionary effects of control strategies and urban } \\
\text { design regulation; }\end{array}$ \\
& 5. & $\begin{array}{l}\text { Integrating zoning into planning and addressing the limitations of } \\
\text { zoning; }\end{array}$ \\
\hline Broad, Substantive & 6. & $\begin{array}{l}\text { Maintaining a commitment to urban design that goes well beyond } \\
\text { elevations and aesthetics to embrace amenity, accessibility, } \\
\text { community, vitality and sustainability; } \\
\text { Design Principles }\end{array}$ \\
7. & $\begin{array}{l}\text { Basing guidelines on generic design principles and contextual } \\
\text { analysis and articulating desired and mandatory outcomes; }\end{array}$ \\
8. & $\begin{array}{l}\text { Not attempting to control all aspects of community design but } \\
\text { accommodating organic spontaneity, vitality, innovation, } \\
\text { pluralism: } \\
\text { not being over-prescriptive; }\end{array}$ \\
\hline 9. & $\begin{array}{l}\text { Identifying clear a priori roles for urban design intervention; } \\
\text { Establishing proper administrative procedures with written } \\
\text { opinions to manage administrative discretion, and with } \\
\text { appropriate appeal mechanisms; }\end{array}$ \\
11. & $\begin{array}{l}\text { Implementing an efficient, constructive and effective permitting } \\
\text { process; }\end{array}$ \\
12. & $\begin{array}{l}\text { Providing appropriate design skills and expertise to support the } \\
\text { review process. }\end{array}$ \\
\hline &
\end{tabular}

Punter [14] incorporates these criteria into a comprehensive framework for design review and development management (Table 1). The twelve principles are grouped under four headings; 
community vision, design planning and zoning, substantive design principles and due process. The principles under the first heading aim to capture the views of the community as a step in generating a comprehensive view to guide development and control. The community vision becomes the reference for all decisions. Secondly, three principles inform development of a suitable process that includes incentives as well as the requirements necessary to regulate for high quality design outcomes. The next three principles address the nature of the relationship between the comprehensive plan and the development industry charged with its implementation. Punter argues that the relationship should be pluralistic and not overbearing in order to allow creative solutions to emerge. Under the final heading four principles address issues of fairness in administering the design control regime.

\section{Does Design Control Help Achieve Well-Liked Streetscapes?}

Concerns about the poor visual quality of many contemporary buildings, as noted in the popular media and by other researchers, must question the effectiveness of design control processes. In a 2003 study, McIndoe [19] audited the effectiveness of two residential character area design guides that had been introduced into the Wellington District Plan. The report reviewed the resource consent process and monitored the architectural merit of the completed project. Following the review of 16 projects implemented over a three year period, the report concluded that the planning rules and associated design guidelines had been effective in achieving desired character outcomes. These results could have been predicted however, as the study investigated the two character areas with the most prescriptive design guides in the District Plan. The report went on to note a positive correlation between the involvement of an architect and a successful design outcome. Similarly, there appeared to be a strong correlation between the absence of a skilled professional and a poor design outcome. The findings of this report thus question the chance of achieving high or even passable outcomes in those projects that do not involve skilled professionals and that are being undertaken in areas with no design guidance.

A year later, Rae [20] reviewed completed projects in the central area of Wellington including the Courtenay and Cuba Character Areas in a similar, subjective review of 20 completed projects. The study concluded that the guidelines were not effective when used in conjunction with controlled activity status under the District Plan. Controlled activity status is where the proposed building or activity essentially cannot be refused, regardless of the aesthetic qualities of the built form. While the earlier study found pre-application consultation to be widespread and beneficial for project outcomes, the second study found early consultations to be ineffective for a variety of reasons. The architects Rae consulted noted that the advice given by Council officers in the early stages was inconsistent and conservative. He also found that when it was undertaken, consultation did not take place at a critical, site-specific analysis stage. Instead, he found, it only took place once preliminary design proposals and economic budgets had been formed. As a consequence, Council urban design input was unable to prompt significant changes, should they be necessary, to improve outcomes. Finally, the research suggested that the discipline specific approach to evaluating projects could have detrimental effect on projects when compared to assessment of proposals in a more holistic manner. 
Rae cited changes in project particulars to satisfy singular and conflicting advice from heritage, traffic, wind, and urban design advisers as having diminished the quality of design outcomes.

While interesting, both of these earlier studies relied on the expertise of the authors to evaluate the design outcomes. The evaluations appear to have been done in the absence of a design framework, instead relying on the expert opinions of each author. Neither study sought input from others, including from members of the public. This would please Scheer [16], who argues that design review should be the exclusive domain of experts trained in the visual arts. In particular, she advocates for the process of design review, if it is undertaken at all, to be fully entrusted to architects, the experts in this field. However, there is little evidence to suggest that such experts speak on behalf of the wider population. Indeed, the little research that has been done to compare the aesthetic preferences of lay people and professionals suggests that there are differences in the way each group evaluates the built environment $[21,22]$. The paper will go on to discuss the findings of a study that was aimed at understanding people's perceptions of urban streetscapes and the individual buildings of which they consist. The study findings are then discussed in relation to the regulatory planning context to firstly understand the extent to which design control leads to well-liked urban streetscapes in Wellington and then to consider areas where improvement could be made.

\section{College Street Case Study}

Late in 2013 a survey of people's visual perceptions of the buildings lining the two sides of College Street in Wellington was conducted. The survey was designed to understand people's preferences for the designs of individual buildings, for the relationships formed between adjacent buildings and for the overall streetscape. The study also sought to identify areas where the aesthetic preferences of members of the different professional groups involved in design review might differ from those of the lay public. College Street was selected for the study for a number of reasons. Primarily, it was seen as a typical urban street, with buildings built to the street edge and from side boundary to side boundary. As it is not a main thoroughfare for motorcars there would be little traffic to distract the attention of people as they made their evaluations of the buildings. As there had been a moderate number of redevelopment projects undertaken over the past 20 years, College Street was also seen as a setting that had been influenced by the city's design control practices.

Survey participants were recruited through notices placed in cafes and other semi-public places throughout a small geographic area. Others were recruited through notices placed in the newsletters of local community groups, business organisations and city council departments. Recruitment sought to ensure participation from a range of demographic backgrounds, including age and occupation. All participants were offered a pre-paid hot drink of their choice when they returned their survey form to one of the local cafes that had agreed to collect these. These efforts led to 75 responses being received.

Initial analysis of the survey data brought forward several streetscape circumstances for further discussion with the participants. Discussion of these issues took place in two focus groups, one comprising trained professionals working in the fields of planning, architecture and urban design and the other only made up of un-trained lay members of the public. The rationale for convening separate groups was to allow the discussions to run more freely, unhampered by a need to consider the views 
of the other group. The College Street case study is part of a larger research project design to identify characteristics in the design of individual buildings and their relationships with others that are valued by the public as well as those that are not. If those that are highly valued can be encouraged through design review and those that diminish aesthetic experience can be avoided then the chance that design control can have a positive effect on the visual qualities of Wellington would be improved.

Preferences for two individual buildings stood out. The first (Figure 1) can be seen to typify the changing context of this part of the city in that it is a building converted from its earlier light industrial use to one that is primarily residential. However, contrary to several other recent developments, it is of a reasonably small scale. The second most liked building is one that accommodates a well-known food market (Figure 2). With the business having been established in the area for more than 20 years, the building has recently been renewed and expanded. The key reasons given by survey respondents for their preferences for these two buildings were the façade details and their overall shapes. Through the focus groups it came to light that these characteristics help both buildings create a positive relationship with the street space and that fenestration helps people relate to the activities taking place inside. The two focus groups were in agreement that these qualities enabled people to forge positive associational meanings for the two buildings.

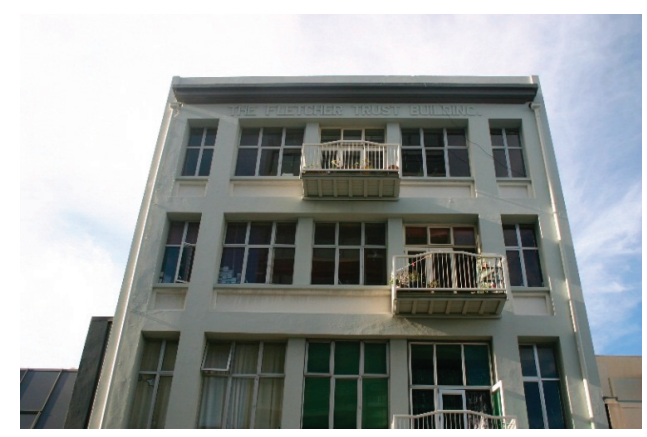

Figure 1. Residential building in College Street. This building has the highest preference rating among the public.

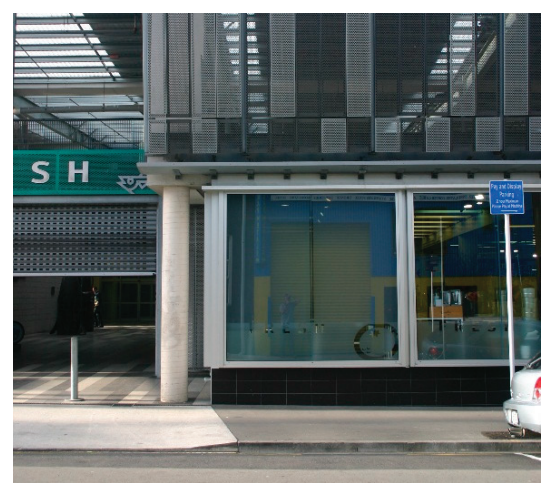

Figure 2. Detail from the street facade of the building of a prominent food retailer, the second best liked building in the street. 
Although several buildings, like the two discussed above, were perceived positively, nearly half the buildings along the street were not liked by the public. The two most disliked buildings were a small, dark coloured structure between the two found to have the greatest appeal (Figure 3) and a multi-storey commercial building at the eastern end of the street (Figure 4). Both were considered impersonal buildings. In the case of the smaller structure, the mirrored glass and dark colour together helped generate these feelings for people. The larger building is large in relation to the street width, has very flat surfaces and finishes and at street level does not forge a relationship with the street because of its blank walls and driveway. Participants in the focus groups identified all these as reasons for the negative evaluation. Both buildings predate the current design control processes and were realised in a planning context where regulation of floor area ratio was the principal method of design regulation.

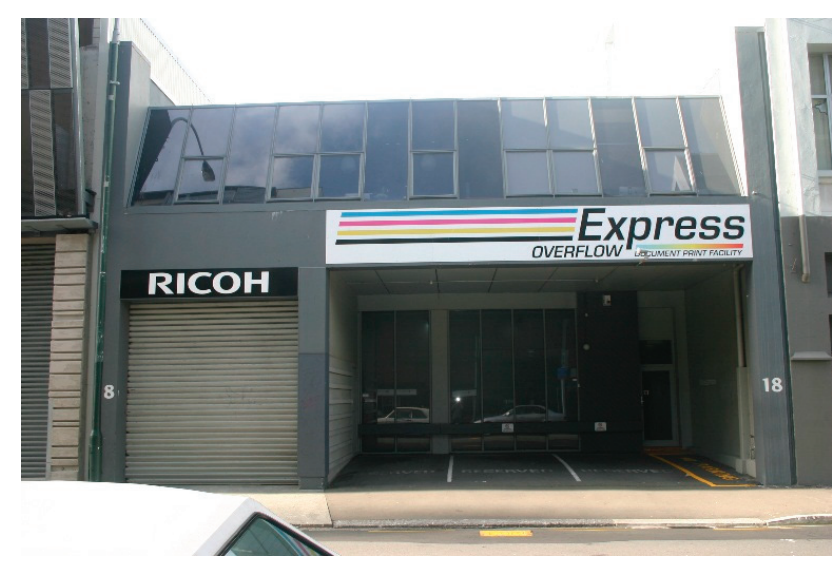

Figure 3. The least liked building in College Street was also judged by survey participants to form a positive relationship with the building to the left.

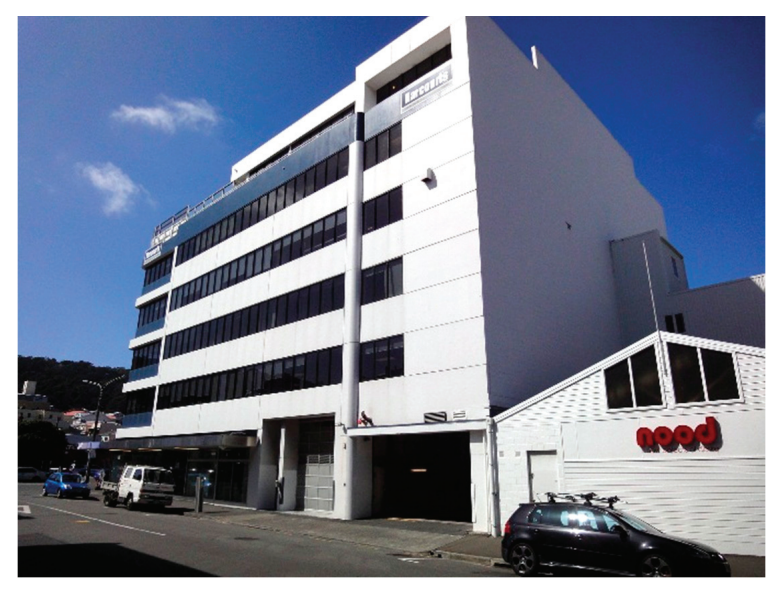

Figure 4. This large commercial building dating to the 1980s is the second least liked building in College Street. 
There appear to be several positive relationships between adjoining buildings along College Street. The relationship between two buildings at the western end of the street (Figure 5) was established decades ago, when warehousing and light industrial activities were dominant. Known in the survey as buildings A \& B, this harmonious relationship is enhanced not only by similarities in height but also by similarities in colour and design expression. As can be seen, the fenestration patterns, façade detailing and alignment between floor levels help create links between the two sites. Not far behind in this preference category is the well-liked relationship between the food market and the least liked building in College Street. This is an interesting result as the two are at opposite ends of the spectrum of public preference. Factors that made this relationship more likable include similarity in building heights, colour and rhythm of façade articulations. These links help overcome differences created by the design approaches taken by the respective designers.

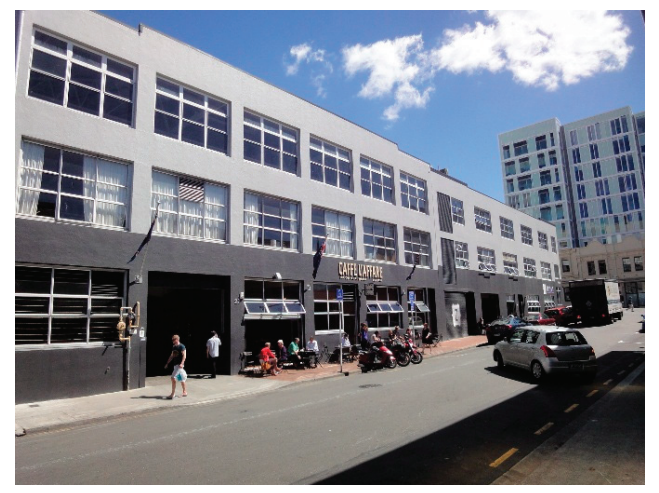

Figure 5. The relationship between these two buildings is seamless and was well-liked by the survey respondents.

The least liked inter-building relationship is considered to be representative of the tensions that arise as the built environment changes to accommodate the growing popularity of an area. One half of this relationship is a large apartment building constructed in 2005, figuratively "riding the wave" of popularity of living close to the city centre. The project amalgamated several titles and was built to $4 \mathrm{~m}$ above the allowable height limit for the zone, which was $27 \mathrm{~m}$. This building was built alongside a single storey, vividly coloured metal workshop with the resultant relationship judged to be very poor by the survey respondents (Figures 6 and 7). Discussions within the professional focus group suggested this poor relationship could be more the perceived "intrusion" of the smaller building, despite it having been there first. In terms of all respondents, the poor relationship was mainly attributed to differences in height and, to a lesser degree, differences in cladding materials. The professional focus group considered that the workshop activity is no longer appropriate for College Street, whereas the lay group felt the opposite. 


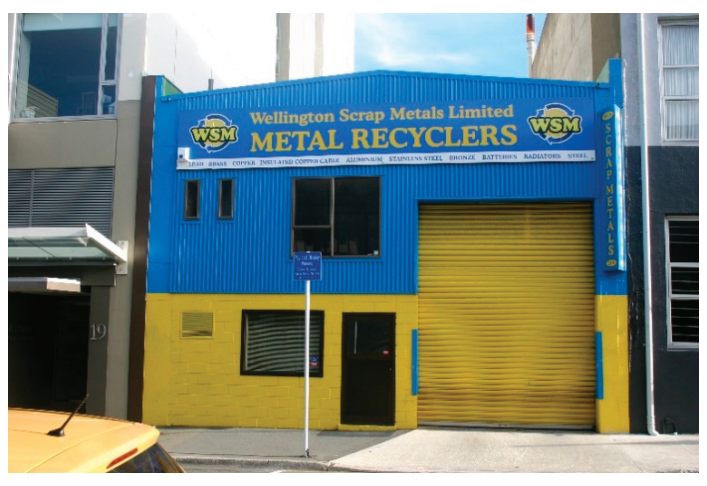

Figure 6. This building for a metal recycling business is typical of the older buildings in College Street. The new apartment building to its left creates a poor relationship between the two.

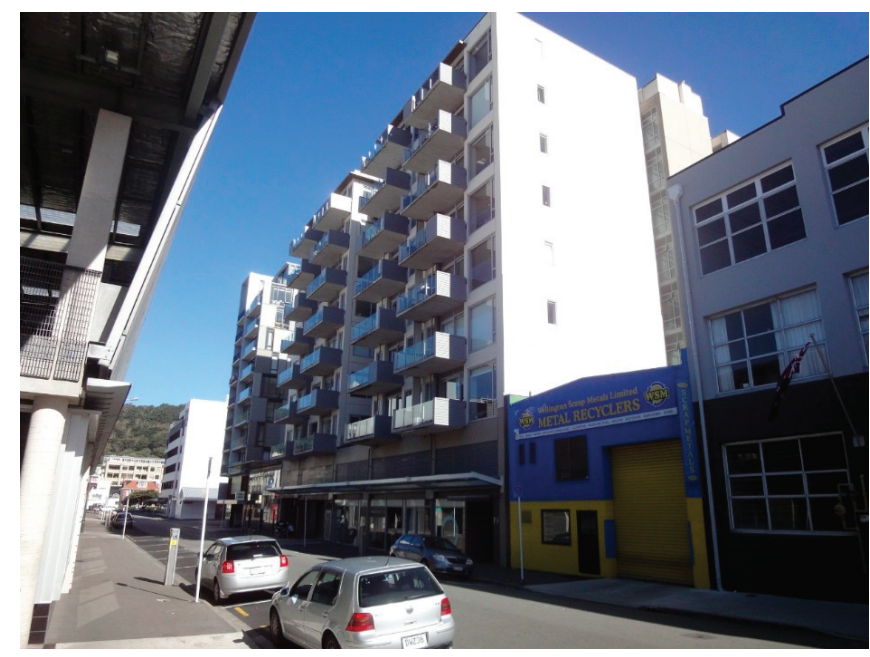

Figure 7. The Trinity Apartments building, in the middle, establishes a poorly regarded relationship with its neighbours. The public also dislike the large blank side wall and the private balconies projecting beyond the site and into the public street space.

The final scale at which perceptions of the College Street environment were examined was across all buildings, in other words the streetscape. Respondents were asked to evaluate the collections of buildings along each side according to relationships between building heights, the three dimensional alignment between facades and the surface designs of these. Definition of these three levels or scales is based on environmental psychology literature [23,24]. The research findings suggest that, while some variation in height along a streetscape can be attractive, people are generally dissatisfied with significant variations within a single street. The southern side of College Street exhibits greater variation and was rated more poorly than the north. Respondents to the survey and participants of both focus groups were united in this opinion. Variations in height were given as evidence of poor 
relationships between buildings and disruptive of aesthetic coherence. While the southern side of the street has undergone more extensive redevelopment than the northern side during the past 20 years, it is perceived negatively at each of the three spatial scales.

As noted, the study was designed to enable comparisons between the aesthetic preferences of those who are directly involved in design review and the lay public, whose views are rarely, if ever, represented. It appears that the opinions of building designers and planners align with the lay public in several areas examined by the survey, with agreement for all but one of the six questions on streetscape. That is, both groups liked or disliked a particular streetscape characteristic, based on a comparison of mean preference scores. Building designers and planners appeared to be marginally more extreme in their ratings, particularly where the mean score fell below neutral. For example, Table 2 shows this group rated the relationships of façade styles on both sides of College Street lower than lay people did, so in this sense they could be considered more critical. When considering the individual buildings and their façade designs similar patterns were observed. In addition to designers and planners being more critical about buildings that were disliked their scores were also generally higher when both groups liked a building or façade design. These patterns align with an earlier study [25] that found professionals were more extreme in their views about streetscapes than lay people. Reasons for this were thought to be training and familiarity, which meant professionals were more confident of their opinions and could express them more clearly.

Table 2. Comparison of mean scores for the streetscape characteristics, on 5-point scale.

\begin{tabular}{lcc}
\hline Streetscape Characteristic & Change Professionals & Lay Public \\
\hline South side & - & - \\
\hline Building Heights & 2.95 & 2.77 \\
Facade Alignment & 2.42 & 2.63 \\
Façade Styles & 2.47 & 2.79 \\
\hline North side & - & - \\
\hline Building Heights & 3.16 & 3.53 \\
Facade Alignment & 2.79 & 3.31 \\
Facade Styles & 2.32 & 2.84 \\
\hline
\end{tabular}

There were however, two areas where there were differences of opinion between these groups. Firstly, the lay public, unlike designers and planners, appeared to like the older, light industrial buildings in College Street, and this was true for every building belonging to the light industrial typology lay. One interpretation of this, supported by the focus group discussion, is that the designer group sees the street character as emergent and considers the older, industrial buildings to be unwanted remnants and possible barriers to further development of the new street character. Secondly, the same group were both more positive about sharp differences in height along the southern side of the street and less positive about the settled pattern of building heights on the northern side. This finding was also supported by the focus group discussion with the possible reasons given for this being a higher wish for variety amongst those directly involved in urban transformation. 
Discussions about the merits of the large apartment building seen in Figure 7 deserve special mention, as its design raised several concerns with the public. Those trained in design and planning were somewhat ambivalent about it but the survey responses suggest that the lay public liked the design. Despite this, members of the layperson's focus group gave several reasons for disliking the building. The key issues were its overall height, the poor relationships formed with the buildings on each side, the large blank side walls, the projecting private balconies over what was felt to be public space (the pavement), and the large areas of glass in the street façade. Interestingly, while people consider fenestration an important contributor to the design, it was generally agreed that the areas of glass are too large for the residential activity in this setting. People were both empathetic to the way glazing can restrict privacy for the buildings' residents as well as cause overheating. While they could appreciate the need for residents to address both concerns by drawing blinds during the day, people also thought the designers could have done more to avoid this.

\section{Discussion}

Building height came out as an important factor influencing people's perceptions of urban streetscapes. The surveys went some way toward identifying this and it was further established during the two focus groups. Where height had a negative influence it was either because this led to an overbearing presence in the street, essentially creating a sense of claustrophobia amongst the respondents, or because excessive variations in height meant large, blank side walls were easily seen. Pronounced differences in height, usually created by the more recent intervention, were considered to be visually awkward and gave an impression of overdevelopment.

More than any other characteristic, height leads to tensions in development and change processes. Height tends to be controlled by area-wide prescriptions. In the urban context, height is where the landowner stands to improve profitability of a redevelopment project. The Wellington design guides do not stress the importance of height relationships between buildings or height in relation to street dimensions. Accordingly new development is prone to push to the limit, and many projects push through it by putting forward an argument that the additional effects of the extra height are minimal. Nevertheless, height differences, such as those that emerge between the building seen in Figure 7 and its neighbours, appear to be recognised by people moving along the street.

The regulatory planning regime of Bergen, Norway is an example of how building height could be addressed in the design review process to limit, if not altogether avoid, potentially negative visual outcomes. The Bergen Regulerings Plan [26] allows for site-specific height controls. Allowable heights are set out in the plan and the current prevailing maximum is $27 \mathrm{~m}$. However, there is also an overarching requirement for new buildings to fit closely with the context and this enables the regulators to limit the height of new buildings to no more than 3 storeys above the neighbouring structure, irrespective of the stated maximum. Another restriction limiting height is the requirement for façades to have a relationship with the street width of no more than 1.1 to 1 . Clearly the emphasis is on the public space surrounding the site rather than giving maximum development potential. While the vision of the plan is a denser, more compact city, the approach seeks to maintain existing building scale relationships while encouraging incremental change. 
Wellington has established blanket allowances for building height, varying from $27 \mathrm{~m}$ in Te Aro to $65 \mathrm{~m}$ in the central area. The only additional factor influencing height appears to be the effect the proposed structure would have on wind patterns at street level. In most circumstances these can be mitigated by the addition of design features, such as sidewalk canopies, that interrupt the flow of wind down to street level. The regulatory context in Wellington does not enable building heights to be controlled in relation to adjoining building heights if they are at or below the blanket height restrictions. And yet, differences in height between adjoining buildings was found to be a factor leading to streetscapes that people dislike. It seems that streetscape outcomes could be enhanced if blanket height controls were to be moderated through consideration of how the proposed heights of new buildings relate to those of adjoining buildings such as is currently done in Bergen.

Visual interest in façade composition appears to be a key success factor. Where the street façade was flat and visually uninteresting, the public tended to judge the building poorly. The survey responses revealed strong correlations between people's preferences for the façade design and their preference for the building overall. The design review process appears to be effective in fostering interesting façade treatments in new buildings. The food market building redevelopment was a good example of this. Uninfluenced by design guidance, most warehouse buildings incorporate flat, utilitarian façade treatment as the emphasis is on a cost efficient enclosure of space. While the food market building in College Street fits with the warehouse typology, the new parts of the building that face onto the street incorporate surface treatments, fenestration and detailing that help generate visual interest. The public responded positively to this, making the street level façade design the best liked of all and ranking it overall the second best liked building in the street.

When it comes to building height new developments that are markedly higher than their neighbours mean the side walls become clearly visible from the street. Side walls are often kept blank for practical, economic and fire protection reasons. While the design guides in the Wellington District Plan seek to avoid uninteresting boundary walls, the recommendations tend to focus on surface treatment and small scale surface modelling. Fundamentally, these remain blank because of a lack of fenestration. It seems that design review has been unable to limit creation of new, visually uninteresting side walls in College Street. This may in part be attributable to a general acceptance by designers and planners that visual interest can be generated in these through application of surface patterns or textures. The public were not willing to accept such treatments as appropriate and expressed their disdain for these additions to College Street through the focus group.

Encroachment into public space by private development was considered to be negative in spatial terms as well as generate negative meanings about the building. The layperson's focus group was particularly concerned about the way the balconies of the large apartment building had been allowed to encroach into public space over the street (Figure 7). When it was pointed out that other buildings, such as the one judged to be favourite amongst all of those in College Street, also had balcony projections into public space, their views did not change. The difference, according to the discussion, was in the number and extent of the encroachments. On the other hand, the professionals' focus group did not view these balcony extensions negatively. The design guide is supportive of the contribution that balconies and other façade articulation can make to design outcome but does not specifically address the matter of where they should be located in relation to the boundary. Encroachments are 
an opportunity for landowners to locate parts of their development on public land if there are reasons why they cannot be fully contained within their site or to accommodate some form of public amenity provided by the landowner, such as the sidewalk canopy. Approval of encroachments is dealt with outside the statutory planning process. Interestingly, where a design outcome relies on articulations extending across into public space there is always a risk the design detail would not be allowed by the encroachment evaluation process.

The literature reminds that people attach meanings to buildings and other parts of the built and natural environment when forming an aesthetic response. This fact has been confirmed, particularly through the focus group discussions where participants provided examples of positive and negative associational meanings when discussing their building preferences. Controlling or guiding design for the meanings people will attach to new developments could however, be problematic, given that meanings are personal, linking to a person's experience as well as to wider cultural or social constructs. Meanings also attach over time and can change over time. While links between certain design attributes and the meanings people relate to them are well known, the design control literature is largely silent on this issue. It may be appropriate then to undertake further research to enable better understanding of how, if at all, design control could be improved by accounting for this dimension of environmental aesthetics.

\section{Conclusions}

This article has discussed regulatory design control from the perspective of public opinion. After establishing the relevance of the appearance of the public realm to the lives of individuals and to society, the paper proceeded to discuss how local government controls the design of new development proposals. Although design control has a long history, only in the past half century has its use become widespread. Design control is not unproblematic and several of the tensions that arise have been identified, particularly tensions around freedom of expression and whose opinions are best to guide decisions about aesthetics.

The question as to whether design control is an effective means by which to ensure well-liked urban streetscapes, has become a research issue. The two earlier studies, both of limited scope, that were conducted in Wellington and that were discussed here did attempt to address this question. However, a significant shortcoming of both is that neither engaged with the public. The research reported in this article did consult with the public in an effort to gauge their opinions on the design quality of individual buildings, of relationships between building and of entire collections or streetscapes. Key factors affecting environmental aesthetic judgment include building height, visual interest and façade composition, the extent to which parts of a development encroach into public space and the meanings people associate with buildings and their details. Addressing the matter of height, it was noted that height is usually prescribed over wide areas with little regard for how this might affect individual relationships between buildings. Land value is clearly tied up with its development potential and allowable height is one of the key factors affecting value. Planning in the city of Bergen, Norway was cited to provide an alternative means for controlling building height. Finally, it was suggested that new research is necessary to understand how associational meanings might be referenced in design control processes. 


\section{Author Contributions}

The research and writing of the paper have been substantially carried out by Morten Gjerde. The research reported in the paper is part of a larger project being conducted by Morten Gjerde as part of his $\mathrm{PhD}$ in urban design. Professor Brenda Vale is supervising the research and contributed directly to the Introduction section of the paper.

\section{Conflicts of Interest}

The authors declare no conflict of interest.

\section{References}

1. Punter, J. From the ill-mannered to the iconic: Design regulation in central Sydney 1947-2002. Town Plan. Rev. 2004, 75, 405-445.

2. Bentley, I. Urban Transformations: Power, People and Urban Design; Routledge: London, UK, 1999; p. 298.

3. Dawson, L. Architecture in the land of the long white cloud suffers from suburban creep and a lack of identity. Archit. Rev. 2010, 227, 33.

4. Pevsner, N. New Zealand. Archit. Rev. 1959, 126, 205-206.

5. Pevsner, N.; Games, S. Pevsner: The Complete Broadcast Talks: Architecture and Art on Radio and Television, 1945-1977; Ashgate Publishing Company: Burlington, VT, USA, 2014.

6. Madanipour, A. Design of Urban Space: An Inquiry into a Socio-Spatial Process; John Wiley \& Sons: Chichester, UK, 1996.

7. Carmona, M.; Magalhaes, C.D.; Edwards, M. Stakeholder views on value and urban design. J. Urban Des. 2002, 7, 145-169.

8. Cuthbert, A.R. The Form of Cities, Political Economy and Urban Design; Blackwell Publishing: Oxford, UK, 2006; p. 290.

9. Wellington City Council. Wellington District Plan Chapter 12: Central Area; Wellington City Council: Wellington, New Zealand, 2013; pp. 1-64.

10. Wellington City Council. Central Area Urban Design Guide; Wellington City Council: Wellington, New Zealand, 2012; pp. 1-25.

11. Carmona, M.; Heath, T.; Oc, T.; Tiesdell, S. Public Places Urban Spaces; Architectural Press/Elsevier: Oxford, UK, 2010; p. 368.

12. Delafons, J. Democracy and Design. In Design Review: Challenging Urban Aesthetic Control; Scheer, B.C., Preiser, W.F.E., Eds.; Chapman \& Hall: New York, NY, USA, 1994; pp. 13-19.

13. Ellin, N. Integral Urbanism; Routledge: New York, NY, USA, 2006; p. 173.

14. Punter, J. Developing urban design as public policy: Best practice principles for design review and development management. J. Urban Des. 2007, 12, 167-202.

15. Nasar, J.; Grannis, P. Design review reviewed. J. Am. Plan. Assoc. 1999, 65, 424-433.

16. Scheer, B.C. Introduction: The Debate on Design Review. In Design Review: Challenging Urban Aesthetic Control; Scheer, B.C., Preiser, W.F.E., Eds.; Chapman \& Hall: New York, NY, USA, 1994; pp. 1-10. 
17. Lai, R.T. Can the Process of Architectural Design Review Withstand Constitutional Scrutiny? In Design Review: Challenging Urban Aesthetic Control; Scheer, B.C., Preiser, W.F.E., Eds.; Chapman \& Hall: New York, NY, USA, 1994; pp. 31-41.

18. George, R.V.; Campbell, M.C. Balancing different interests in aesthetic controls. J. Plan. Educ. Res. 2000, 20, 163-175.

19. McIndoe, G. Mt. Victoria and Thorndon Residential Character Implementation Audit; Wellington City Council: Wellington, New Zealand, 2003; p. 20.

20. Higgins, M. Urban design and the planning system in Aotearoa-New Zealand: Disjuncture or convergence? Urban Des. Int. 2010, 15, 1-21.

21. Groat, L.N. Carbuncles, Columns and Pyramids: Lay and Expert Evaluations of Contextual Design Strategies. In Design Review: Challenging Urban Aesthetic Control; Scheer, B.C., Preiser, W.F.E., Eds.; Chapman \& Hall: New York, NY, USA, 1994; pp. 156-164.

22. Stamps, A.E. Demographic effects in environmental aesthetics: A meta-analysis. J. Plan. Lit. 1999, 14, 155-175.

23. Stamps, A.E. Psychology and the Aesthetics of the Built Environment; Kluwer Academic Publishers Group: Dordrecht, The Netherlands, 2000; p. 320.

24. Nasar, J.L. Environmental Aesthetics: Theory, Research, and Applications; Nasar, J.L., Ed.; Cambridge University Press: Cambridge, UK, 1988; p. 529.

25. Gjerde, M. Visual evaluation of urban streetscapes: How do public preferences reconcile with those held by experts. Urban Des. Int. 2011, 16, 153-161.

26. Bergen Kommune. Kommunedelplan Sentrum; Bergen Kommune: Bergen, Norway, 2002; p. 63. (In Norwegian) 


\title{
A Framework of Thermal Sensitive Urban Design Benchmarks: Potentiating the Longevity of Auckland's Public Realm
}

\section{Andre Santos Nouri}

\begin{abstract}
One of the key objectives of contemporary urban design is to ensure the quality and activity within urban public spaces. Presented as a progressively emerging paradigm in this process, the effects of urban climatology are increasingly elucidating the need for further climate responsive environments. Moreover, this interest is one that shall increase along with the progression of climate change effects upon outdoor environments. Nevertheless, it is often that climatic assessments lack bottom-up climatic indicators, tools and practical benchmarks. As a result, this obstructs local decision making, and practices of localised adaptive design. In an effort to address such discrepancies, this paper launches a framework of international precedents of built and conceptual projects that address thermal comfort levels in public spaces. This organisation will be cross-referenced with theory that supports its structure and typological division. With Auckland as the focal case study, the solutions that are extracted from the framework will be scrutinised in order to shape new potential measures, and launch new considerations in Auckland's local policy and design guidelines. In this way, microclimatic concerns are hence framed into an opportunity to potentiate the use and longevity of Auckland's public realm.
\end{abstract}

Reprinted from Buildings. Cite as: Nouri, A.S. A Framework of Thermal Sensitive Urban Design Benchmarks: Potentiating the Longevity of Auckland's Public Realm. Buildings 2015, 5, 252-281.

\section{Introduction}

Before reaching the mid-twenty-first century milestone, it is expected that population, urban density and $\mathrm{CO}_{2}$ emissions shall significantly increase in Auckland. Consequently, sustainable decision making becomes fundamental in amalgamation with the council's aim to make Auckland the world's most liveable city by 2040 [1]. In conjugation with this expansion, the practice of urban design is also presented with the interdisciplinary challenge of preparing for impending local "risk factors" as a result of climate change.

Although knowledge regarding outdoor thermal comfort has grown in recent years, its assimilation with climate responsive urban design has been considerably limited. As a result, local decision makers and designers often lack the design indicators and benchmarks to: (1) address existing microclimatic implications in public space design; and more prominently; (2) prepare for the invigoration of these respective insinuations as a result of climate change. With the aim of tackling such discrepancies, and through a Research for Design approach, this article reviews a range of international solutions that address similar microclimatic constraints similar to those found in Auckland. 
This investigation is launched as part of an ongoing funded doctoral research with the title "City Identity in Uncertain Climate Change Horizons: A Research Approach for Microclimatic Urban Design in Public Spaces". As part of a chapter that explores the interaction between public space design and thermal comfort levels, this article launches a demonstrative case study on how a framework of thermal sensitive urban design can introduce new deliberations in both local policy and design guidelines for climate-responsive public spaces within the city of Auckland.

\section{New Zealand's Climate and Future Implications}

As a means to identify a basis for climatic regionalisation and comprehend variables from Global Circulation Models (GCMs) outputs, the Köppen-Geiger (KG) climate classification system has classified New Zealand as a Temperate/Mesothermal climate. More specifically, and supported by a top-down outlook, the updated world map of the KG system classifies this genre of climate as "Cfb", meaning a "Maritime temperate climate" or "Oceanic climate" [2]. Resultantly, this is concomitant with temperature fluctuations associated with large-scale climate patterns over the Southern Hemisphere and the Pacific Ocean. These meteorological phenomena have a temporal timeframe that can range from seasons to decades, such as the El Nino-Southern Oscillation (ENSO) and the Interdecadal Pacific Oscillation (IPO). Each of these oscillations can influence seasonal temperatures, wind patterns, and precipitation levels [3]. Consequentially, this natural variability invariably blurs the superimposition with long-term human-induced climate change trends.

Based on the disseminated figures from the National Institute of Water and Atmosphere (NIWA), New Zealand does not have a broad temperature range, and it lacks extreme values that are commonly found in most continental climates. Moreover, and due to being located in the Southern Hemisphere, northern cities experience higher temperatures throughout the year. As shown in Table 1, Auckland is one of these cities and is one of the warmest city centres in New Zealand.

Due to being encircled by the Pacific Ocean, the country is expected to experience a delay in mean temperature change in comparison to global averages over the medium term [7]. This delay notwithstanding, national climate change projections indicate "very confidently" that until the end of the century there shall be: (1) a temperature increase of between 0.2 and $2.0^{\circ} \mathrm{C}$ by 2040 , and between 0.7 and $5.1^{\circ} \mathrm{C}$ by 2090; (2) an increased frequency of high temperatures; and (3) an accelerated rate of temperature increase in comparison to the temperature patterns recorded for the twentieth century [3].

At a regional scale, and returning to the case of Auckland, it is a projected that by 2100 , there will be at least $40+$ extra "hot days" where maximum temperatures surpass $25{ }^{\circ} \mathrm{C}$ [7]. In retrospect with current values shown in Table 1 , this implies that there will be a $200 \%$ increase in annual "hot days".

Furthermore, it is also worth noting that due to the proximate "ozone-hole", the county's peak Ultra Violet (UV) intensities can be $40 \%$ higher in comparison to similar latitudes in the northern hemisphere (e.g., the Mediterranean area). Although a UV index of 10 is already considered extreme, this index value can exceed 13 during the summer in cities such as Auckland. 
Table 1. Summary of climate information for the six main City Centres in New Zealand (* Average Relative Humidity (RH) levels were taken at 9 am, hence these figures vary approximately if combined with afternoon $\mathrm{RH}$ levels-For the case of Auckland, this would decrease annual RH approximately to $76 \%$. $* *$ Annual count of "hot days" where temperatures exceeded $25{ }^{\circ} \mathrm{C}$-values presented are annual averages since mid-twentieth century. Wet-days, sunshine, temperature, wind speed, and average relative humidity data are mean values from the 1981-2010 period). Adapted with permission from NIWA [4-6].

\begin{tabular}{|c|c|c|c|c|c|c|c|c|}
\hline \multirow{2}{*}{ Location } & \multirow{2}{*}{$\begin{array}{c}\text { Mean Relative } \\
\text { Humidity } \\
\%(9 \text { am *) }\end{array}$} & \multirow{2}{*}{$\begin{array}{l}\text { Wet-Days } \\
\geq 1.0 \mathrm{~mm}\end{array}$} & \multirow{2}{*}{$\begin{array}{c}\text { Sunshine } \\
\text { Hours }\end{array}$} & \multicolumn{4}{|c|}{ Temperature } & \multirow{2}{*}{$\begin{array}{c}\text { Wind } \\
\text { Speed } \\
\text { Av. Wind } \\
\text { Speed m/s }\end{array}$} \\
\hline & & & & $\begin{array}{c}\text { Mean } \\
{ }^{\circ} \mathrm{C} \\
\end{array}$ & $\begin{array}{c}\text { Mean } \\
\operatorname{Max}^{\circ} \mathbf{C}\end{array}$ & $\begin{array}{c}\text { Mean } \\
\operatorname{Min}{ }^{\circ} \mathbf{C}\end{array}$ & $\begin{array}{c}\text { Hot Days (Max } \\
\left.\text { Temp. }{ }^{* *}>25^{\circ} \mathrm{C}\right)\end{array}$ & \\
\hline Auckland & $82.3 *$ & 137 & 2060 & 15.1 & 19.0 & 11.3 & 21 & 4.72 \\
\hline Tauranga & 78.8 & 111 & 2260 & 14.5 & 19.1 & 10.4 & 21 & 4.44 \\
\hline Hamilton & 85.0 & 129 & 2009 & 13.7 & 18.9 & 8.7 & 28 & 3.33 \\
\hline Wellington & 82.3 & 123 & 2065 & 12.8 & 15.9 & 9.9 & 3 & 6.11 \\
\hline Christchurch & 85.1 & 85 & 2100 & 12.1 & 17.2 & 7.3 & 21 & 4.17 \\
\hline Dunedin & 73.1 & 124 & 1585 & 11.0 & 14.7 & 7.6 & 8 & 4.17 \\
\hline
\end{tabular}

In this light, perspectives towards the future adjoin the opportunity to deliberate upon more frequent and intense temperature levels in the city. Consequently, contemporary urban design embraces the need to certify that thermal comfort levels are addressed in the intricate balance between the urban microclimate, human characteristics, and the use of public spaces [8]. Regrettably, although the characteristics of urban climate have been well studied in the past two decades, there is little association with the possible application of physical urban design interventions.

In the scope of public space design, urban climatology is recognised as an essential component in the thermal analysis of open spaces in order to improve the collaboration between thermal physical attributes and the social environment as a whole [9]. Naturally, when presented with the risk factor of climate change, urban climate distribution becomes increasingly more important, and issues such as solar radiation, ventilation and heat islands present new challenges for local adaptation/intervention. More specifically to the local scale, the thermal environmental effects upon humans can be determined with the aid of thermal indices based on the energy balance of the human body. Thermal indices include considerations upon meteorological factors such as air temperature, air humidity, wind velocity and short/long wave radiation that affects humans thermo-physiologically in outdoor environments [10] Examples of such indices will be presented in Section 3.

As determined by Olgyay [11], thermal comfort is the balance of various microclimatic thermal stimuli. Consequently, if a stimuli such as ambient temperatures increase, then the balance of human comfort shall inevitably shift and require measures that cool ambient temperatures through the use of wind, shade, and/or evaporative cooling. Adjacently, the urban morphology can lead to local heat islands and thus require similar interventions to alleviate thermal stress levels. In the case of Auckland, although it shall face more attenuated climatic effects in comparison to global 
averages, its Unitary Plan (UP) invariably recognises the need to "increase the resilience of Auckland's communities and natural and physical resources to the anticipated effects of climate change such as (...) more frequent and extreme weather events." ([1], p. 174). Moreover, and presented as a "Quality urban growth objective" in the UP, there is also an ardent interest in a "high quality network of public open spaces and recreation facilities that enhances quality of life (...) and contributes positively to Auckland's unique identity." ([1], p. 178). Given the recognition of future climatic implications, and the importance of Auckland's public spaces, urban resilience and adaptability becomes a fertile scope of opportunity for local action. In this way, local decision makers and designers are hence tasked with considering the long-term longevity of the city's public realm that shall determinedly face climatic hurdles until the end of the century.

\section{Urban Design Case Studies and Benchmarks}

Since the turn of the century, the maturing climate change adaptation agenda has gained a new weight, and has instigated local decision makers and designers to search for measures to address local "risk factors" [12]. This early, yet developing bottom-up perspective, is one that explores how urban design and climatic adaptation can tackle meteorological implications through an interdisciplinary approach.

This section explores existing bioclimatic case studies that can potentially be used as benchmarks to address the impending threat of increased temperatures and heatwaves upon Auckland's public realm. In order to facilitate the typological differentiation between the discussed measures, and adapted from authors such as [13,14], four principal categories have been respectively established: (1) trees and vegetation; (2) shelter canopies; (3) materiality; and lastly (4) water and vapour systems. Of these four, a slightly greater emphasis shall be given to the categories of materiality and water/vapour systems. The reason for this is interlaced with their later appearance in urban design, and the considerable amount of scientific incongruity associated with their successful effects upon thermal comfort levels. During the ensuing section, existing international practices and/or projects shall be viewed as an opportunity to shape new potential measures, and additionally launch new considerations in Auckland's local regulatory and nonregulatory design guidelines. Given that Auckland shall experience meteorological aggravations such as increased hot days, the disclosed measures shall focus on how this can be overcome. With this objective in mind, and also taking into account the proposed alterations to microclimatic factors such as shading and wind patterns, the benefits of the projects shall be discussed in terms of reducing overall ambient temperatures (i.e., $\mathrm{K}$ ).

\subsection{Trees and Vegetation}

When considering the long term environmental adaptability of a city, there is a consensus that vegetation can significantly contribute to the improvement of the urban microclimate due to its ability to reduce air temperature through direct shading (Although there is still a limited amount of research pertaining to the direct effect of vegetative shading at pedestrian levels, the doctoral thesis of Ana Almeida suggests that "trees, just like other green spaces inserted in edified areas can 
lower temperatures by approximately $\left.3{ }^{\circ} C^{\prime \prime}\right)$, ([15], p. 54) and evapotranspiration. More specifically, these processes induce the decrease of radiant temperature, influence wind patterns (both in velocity and direction), air regeneration (such as $\mathrm{CO}_{2}$ absorption), and filter both dust particles and noise. Moreover, and besides these environmental attributes, vegetation can also provide additional psychological benefits to humans through aesthetic, emotional and physiological responses [16].

In existing studies relating to vegetation as a form of microclimatic control in urban open spaces, four principal green "structures" can be identified: covering vegetation, isolated trees, and groves or lines of trees [17]. However, it is important to note that unlike inanimate devices, trees can change their dimension and degree of opacity during each season, and also during their lifetime. As a result, and although variations among trees may be considered aesthetically pleasing, the designer/planner needs to be aware of the shading pattern produced [14,17]. In terms of seasonal timeframes, there needs to be a consideration of: (1) how shade patterns can be provided in the summer when/where needed; (2) how solar penetration can be enticed during the winter period when/where needed; and (3) which specific trees provide these desired effects during the pertinent time of year.

Regrettably, and although recognized as an effective way to alleviate higher temperatures, the incorporation of these vegetation reflections upon thermal sensitive urban design is limited. Yet, authors such as Shashua-Bar, et al. [18] have explored the potential of passive cooling through the modelling of design options on outdoor thermal comfort in urban streets in the shade of both trees and buildings (Case \#1). In their research, they analysed how street design scenarios benefited from the combination of vegetation with other measures in order to attenuate thermal comfort levels during the summer. To do so, the biometeorological index Physiologically Equivalent Temperature (PET) was used in order to assess levels in a typical street of Athens. Four theoretical design cases were undertaken: (1) increasing the tree's canopy coverage area from its actual net level of $7.8 \%$ to $50 \%$; (2) reducing traffic load from two lanes to one and thus approximately reducing 1500 vehicles down to 750 per hour; (3) increasing the albedo of the adjacent side walls from the measured 0.4 to 0.7 by implementing lighter colours; and lastly; and (4) deepening the open space by increasing the aspect ratio (height/width proportions) from the existing 0.42 to 0.66 through elevating the side buildings by two additional floors (approximately $6 \mathrm{~m}$ ) [18]. The results of the study illustrated that the most successful passive design solution was that of increasing the vegetative canopy coverage that resulted in a decrease of $1.8 \mathrm{~K}$ during noon hours. This is particularly interesting when comparing to the more drastic and expensive option of increasing the aspect ratio, which achieved a similar decrease of $1.9 \mathrm{~K}$.

\subsubsection{Application in Cooler Climates and Overcoming Risks of Overshading}

Conversely, when applying this to Auckland, it is clear that, due to its more temperate climate, considerations would need to be made upon the issue of overshading. Nevertheless, the constructed Parisian climate sensitive redevelopment-project, "Place de la Republique" (also located in the KG classification of "Cfb") can be used as a practical example of how these issues can be resolved (Case \#2). Trevelo \& Viger-Kohler Architects and Urbanists aimed at addressing the thermal 
comfort and Urban Heat Island (UHI) effect within the now largest pedestrian square in Paris. Today, an overall 134 deciduous plane (Platanacease) trees and 18 deciduous honey locust (Fabaceae) trees encircle both the new perimeter and central area. Unlike the common segregation between vegetation and the thermal design of the public space, and in line with their environmental approach, the square is "comfortable as a result of a strategy that is at once urban, landscaped and architectural" ([19], p. 7). More specifically, this strategy consists of implementing measures that prevent the square from becoming a "heat island", namely by: (1) increasing planting and creating a unit of vegetation to provide maximum mass effect; (2) allowing the sun to penetrate and position the pedestrian areas in the sunniest areas; (3) blocking the colder winter winds by thickening the vegetation at the north of the square; and just as importantly, (4) linking the presence of vegetation in order to consolidate usage dynamics in the square to suit prevailing conditions [19].

\subsubsection{Lessons for Auckland's CBD}

Returning to the specific case of Auckland (and furthermore considering the temporal timeframe of 2040), the city is challenged with considering the specific implications of how vegetation can be appropriately introduced in order attenuate thermal comfort levels. Furthermore, and considering the responses from agencies such as the Auckland Regional Public Health Service (ARPHS) to the UP, the effects of UHI need to be considered further, especially given the future increases of both urban density and climatological impacts [20].

Respectively, and strengthened by the case studies presented in this first section, it is suggested that future projects (as an example, this will be particularly relevant in "Move 6" of the Auckland's Masterplan; that suggests an ecological "Green Link Network" that shall insert a "wave" of green vegetation to enhance the environmental sustainability at street level as part of the redesign of Victoria Street and adjacent open spaces) must consider vegetative: (1) annual shading patterns; (2) change in dimension and degree of opacity; (3) contributions to decreasing the UHI effect; and lastly (4) effects upon the activity threads, and usage of the urban realm in accordance with prevalent microclimatic conditions.

\subsection{Shelter Canopies}

When addressing canopies or roof structures in urban open spaces, the air temperature underneath the structure is predominantly affected by the existing solar exposure of the space. In turn, this directly relates upon the geometry of the structure, components, and the properties of its construction materials. The respective radiant temperature is interrelated to the temperature of the inner surface of the roof, which can be either lower or higher than the air temperature of the space underneath. Furthermore, the air velocity in the spaces underneath depends ultimately on the incoming wind/air patterns that are allowed to enter/penetrate the area.

In the case of Auckland's Central Business District (CBD), passive strategies to decrease solar radiation through shelter canopies are already present. Yet, and using Queen Street as an instance, most measures are only applied upon commercialised street sidewalks, and not within local open public spaces. With hindsight, civic spaces such as Aotea Square, Freyberg Square, and Queen 
Elizabeth Square are currently recognised by the UP as "becoming increasingly important as Auckland's centres intensify and access to high-amenity open space is needed for residents" ([1], p. 58). Perhaps due to the fear of overshading, these spaces do not accommodate passive structures that decrease and/or attenuate local solar exposure. Although this is beneficial during the winter months (i.e., June to August), there is limited shading that would otherwise entice the increased usage of these spaces during the summer. Interestingly, prominent studies in the use of New York's public spaces suggest that "the days that bring out the peak crowds on plazas are not the sparkling sunny days with temperatures in the [low $\left.20^{\circ} \mathrm{Cs}\right]$ (...) it is the hot, muggy days, sunny or overcast, the kind that could be expected to make people want to stay inside and be air conditioned, when you will find the peak numbers outside" ([21], p. 44). Following this line of thought, the interplay of canopies regarding the provision of choice between experiencing sun, shade, or in-between areas becomes indispensable.

However, before any intervention can be considered, there needs to be a local and annual understanding of: (1) the patterns of existing solar radiation exposure (usually measured in hours); (2) the shadows that are cast from on-site elements (i.e., such as vegetation and amenities); (3) the shadows that are cast from off-site elements (i.e., such as contiguous structures and buildings); and (4) existing encircling wind patterns.

\subsubsection{Permanent and Ephemeral Approaches to Passive Cooling}

Once established, thermal sensitive urban design can present the opportunity to improve the current thermal response of these spaces in both colder and hotter months. More prominently, the long-term response to increased higher temperature and frequency in Auckland can be tackled through a precautious approach. In this scope, both permanent and temporary measures can be considered to increase local shading opportunities.

In the pursuit for case studies that have used shelter canopies in their bioclimatic approach to the public realm, permanent solutions can be extracted from the entries from the European competition "Re-Think-Athens". Although situated and tempered for a hotter climate (i.e., "Csa" in the KG classification), many of the proposed measures can be adapted to Auckland's public realm and enclosing climate. The winning proposal "One Step Beyond" (Case \#3) by OKRA Landscape Architects based their design upon a pedestrian-orientated space that incorporated contemporary ideas of climate control in order to address thermal comfort through microclimatic attenuation [22]. In one of the public spaces within the redevelopment proposal (Omonia square), a limited amount of shelter canopies were introduced into the space. Although the four canopy structures shade less than $10 \%$ of the total area of the public space, they are strategically placed on the extremities of the square alongside kiosks and food/beverage units. As a result, the risk of over-shading during the winter is null, nonetheless, effective shading is still accomplished during the summer in strategic locations.

Another noteworthy and runner up entry was the submission of ABM Architects "Activity Tree" that, although it shall inevitably remain as a concept, offers, nevertheless, valuable precedents in terms of shelter canopies (Case \#4). Established through an in-depth site analysis, the zones which would require protection/attenuation from solar radiation were to be protected by 
“Activity/Bioclimatic Trees". These canopies would cast shadows in specific areas and would serve as an advanced bioclimatic device that would be able to capture energy and water. Through a detailed analysis of sun patterns, and in order to permit solar penetration during the winter, the structural celosias system allowed the winter sun to penetrate the covered spaces.

Additionally, it is also worth noting that short-term interventions also find their niche in this category of thermal responsive urban design. Here, design can also be interlinked with ephemeral projects in order to tackle periods of higher temperatures and/or heat waves in public spaces. As an example of an Ephemeral Thermal Comfort Solution (ETCS), Ecosistema Urbano Architects launched the conceptual project "This is not an Umbrella" (Case \#5). Although a simple concept, it is a lightweight and low cost solution, which enables the climatic control of a large outdoor space. The proposal is thought of as a citizen participation action that uses 1500 hanging umbrellas to shade the patio of the Spanish Matadero Contemporary Art Centre. Lastly and also erected in the exterior of a contemporary Art Centre in New York, Architects built an ETCS to provide relief from the hot summer weather. With the use of a precise 3D model, the "Canopy" was built with freshly cut green bamboo that provided armature for four different microclimates, which were also attenuated with three different water systems (Case \#6).

\subsubsection{Lessons for Auckland's CBD}

Resultantly, both long-term and ETCS canopies find their role in attenuating urban thermal comfort levels. In the case of Auckland, this genre of intervention should be used to enhance availability of choice between exposed and shaded areas throughout the year. Moreover, the necessity of providing such choice shall increase along with the projected escalation of annual hot days in light of climate change. However, in order to avoid over-shading the city's public realm, careful analysis of existing solar patterns, shadows, and wind configurations is required. As demonstrated in the cases disclosed in this section, the tempering of thermal comfort levels can only be accomplished through the understanding of local annual microclimatic implications.

Figure 1 exhibits a conceptual bioclimatic intervention in Queen Elizabeth Square that, amongst other strategies, uses shelter canopies to improve thermal comfort levels during the summer. Photographed during the summer period, Figure 1a demonstrates a lack of activity and, moreover, a high exposure to solar radiation. Although there is vegetation on one side, the central square lacks the means to attenuate solar intensities and temperatures during the hotter months. As a result, Figure $1 \mathrm{~b}$ shows a conceptual intervention that would be based around the use of: (i) mobile shelter canopies that could serve as temporary shading measures for the café's seating area; and (ii) a central water feature that would be turned on during hotter days, and which could entice an increase of foot fall/activity threads within the centre of the square. This simplistic conceptual intervention demonstrates an exploration of how microclimatic interventions could not only improve thermal comfort levels but also potentially increase activity threads through climate responsive public space design. 


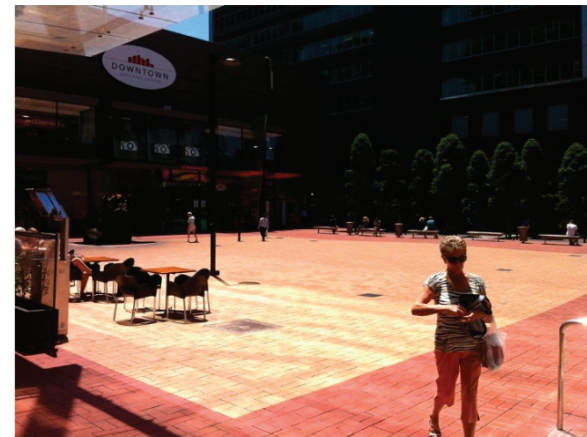

(a)

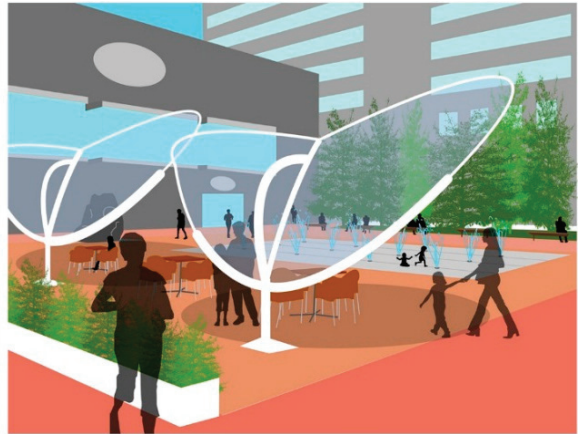

(b)

Figure 1. Conceptual intervention in Queen Elizabeth Square (a) Existing square; (b) Square post bioclimatic intervention. Source: Author's rendering.

\subsection{Materiality}

The phenomena of UHI effects are becoming increasingly more intense in cities, and are consequentially coercing modifications upon the urban microclimate. As a direct result, the temperature disparities between urban and suburban/rural areas are continually increasing. This results from a positive balance within the urban environment due to increased heat gains consequential to the high absorption of solar radiation, the release of urban anthropogenic heat, decreased radiant heat loss under the Urban Canyon Layer (UCL), and lower wind velocities.

Through the design of both the public realm and public spaces, the global scientific community has already made significant progress in the endeavour to counterbalance UHI effects. More specifically, the proposed mitigation techniques and technologies involve the use of "cool materials" which present both high reflectivity and thermal emissivity values [23].

In this light, the application of such materials upon urban surfaces finds its niche within the design and conceptualization of the urban realm. Early investigations dating back to the 20th century already argued that the impact of pavements upon UHI was substantial, and furthermore played a considerable role in attenuating the overall urban thermal balance. The specific thermal balance of pavements is determined by the amount of: (i) absorbed solar radiation; (ii) emitted infrared radiation; (iii) heat transfer as a result of convection into the atmosphere; (iv) heat storage by the mass of the material; (v) heat conducted back into the ground; (vi) evaporation or condensation when latent heat phenomena are present; and lastly, (vii) inflicting anthropogenic heat through urban activity such as vehicular traffic upon roads [24,25].

Returning to the design of public spaces, decreasing the surface temperatures of elements such as pavements may thus significantly improve the thermal conditions in spaces suffering from elevated atmospheric temperatures. In practical terms, this can be achieved through the replacement of conventional paving surfaces that present higher surface temperatures during warmer periods. 


\subsubsection{The Impact of Material Reflectivity and Emissivity upon Ambient Temperature}

Solar reflectivity (i.e., albedo) of the respective material is, in general terms, influenced mostly by the colour of the material (another influencing factor can be the roughness of its surface, whereby those that present smoother/flatter surfaces are those with lower surface temperatures). In most cases, when a material is made of a lighter colour, it presents a higher albedo due to its lower absorptivity to the visual spectrum of solar radiation. In the study undertaken by Doulos, Santamouris, and Livada [26], numerous investigations were performed to correlate the impact of both the colour and roughness of various paving materials and their resultant surface temperatures. As part of their disseminated outputs, the investigation indicated measured air temperature, hence demonstrating the relationship between that of surface temperature and air temperature. The results of the study demonstrated that darker materials presented a significantly higher surface temperature comparatively to that of ambient temperature; thus, it can be concluded that this leads to the surfaces having a warming effect upon local ambient temperature. On the other hand, the surface temperatures of white concrete and marble are more analogous to local air temperature, especially in the case of white marble [26]. Similarly, the remainder of such temperatures in darker common materials can thus lead to elevated temperatures after peak hours due to convective heat dissipation from the pavement. This occurrence is a direct result of a combination of both low reflectivity and emissivity, whereby the radiation absorbed by the material is retained within its mass, and posteriorly dissipated back into the atmosphere, hence leading to elevating temperatures even after peak hours (materials with high emissivity correspond to good emitters of long wave radiation, and can, moreover, easily release the absorbed energy from solar radiation. For this reason, materials with lower emissivity rates are the principal reason for increased surface temperatures during the night as shown in [27]). Disseminated within the international scientific arena, further theoretical (and to some extent empirical) studies, both at local and regional scales, have enforced such relationships between materiality and surface/ambient temperatures [28,29].

\subsubsection{Lessons Learnt from the Direct Application of Materials in Bioclimatic Projects}

Analogous to the discussed global studies regarding the thermal benefits of high albedo and emissivity materials in contemporary cities, several bioclimatic projects have already incorporated such approaches to cool local temperatures and attenuate UHI effects.

The characteristics of such projects are demonstrated in Table 2, where it is worth noting that most projects are located in the same KG climate classification system (i.e., "Csa"). Case \#7 deals with the bioclimatic rehabilitation of a central area in the city of Marousi in Athens, and is currently under construction. Following the results of a microclimatic assessment, both innovative architectural and environmental techniques were used to attenuate local thermal comfort levels. Before the project was commenced, the area was known for its: (i) increased ambient temperatures during the summer period, and for its generally unsatisfactory thermal comfort levels; (ii) extensive use of black asphalt in streets, and the use of dark concrete tiles upon pavements; and (iii) susceptibility to medium intensities of UHI effects [30]. To overcome such issues, and to substantially improve the local microclimate, a bioclimatic plan was thus launched. 


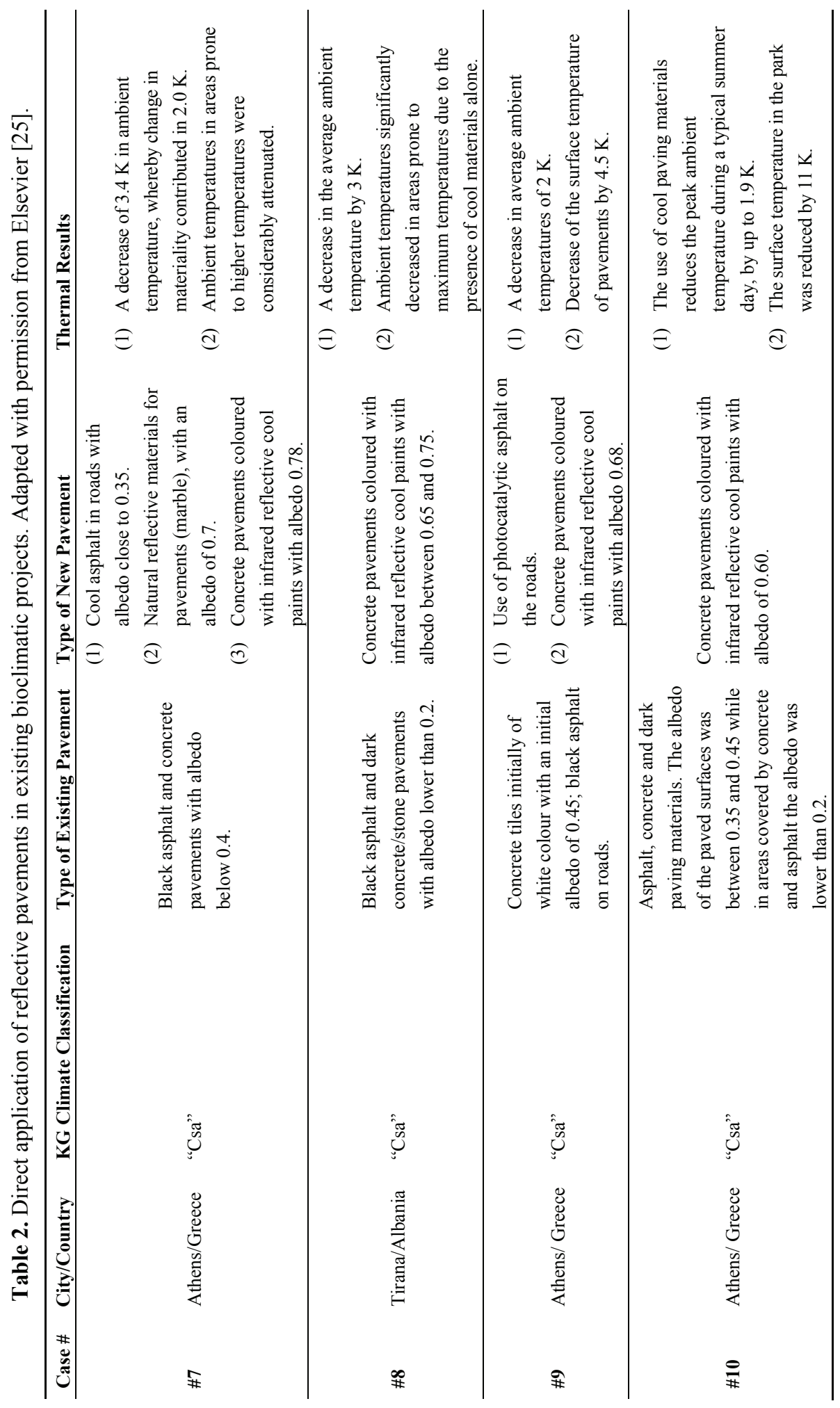




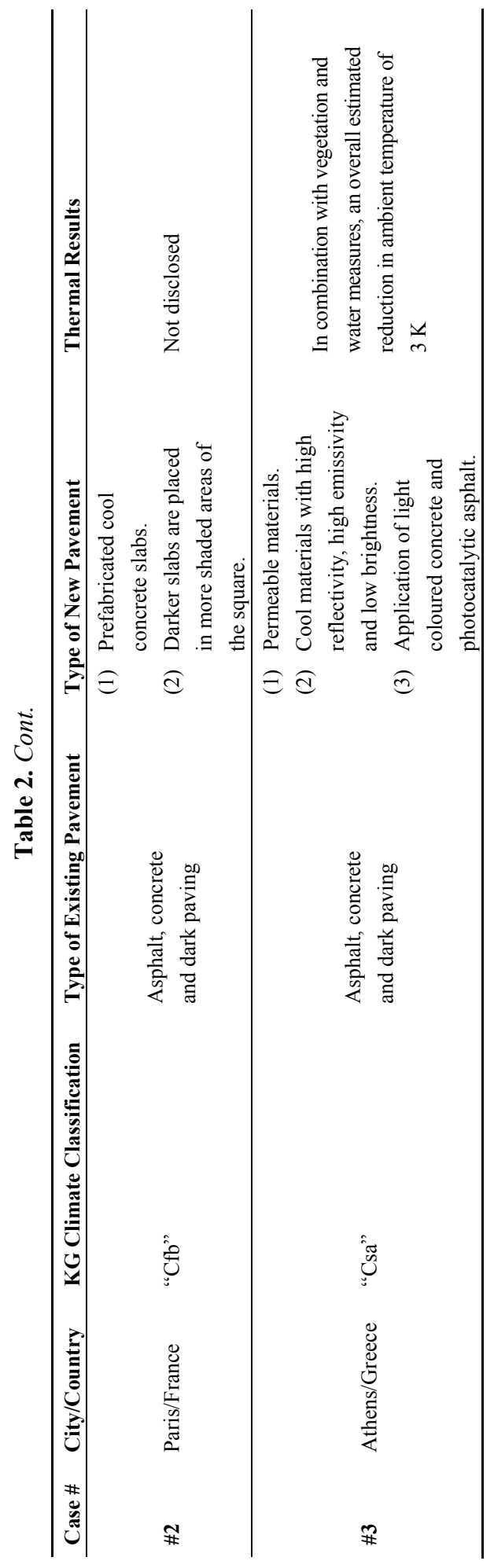


In order to attenuate the temperature of the public realm, the proposed plan would integrate bioclimatic techniques with public space design through introducing: (i) an increase of vegetation in the area by planting new trees; (ii) the use of solar devices to improve/enhance shading; (iii) the use of water that would function as a cool sink; (iv) the use of earth to air heat exchangers to dissipate the excess urban heat to the ground; (v) photovoltaic panels; and finally, (vi) the use of materials with appropriate thermal properties such as cool materials.

With the exception of the earth to heat exchangers, most of the measures specifically considered the influences they would have upon the surface temperature of local materials. The thermal results of the project are shown in Table 2. The installed measures included the: (i) Extension of shading and solar control, aimed at reducing surface temperatures of pavements; (ii) use of tall trees and pergolas in order to improve the efficiency of local evapotranspiration; (iii) use of light coloured materials to decease the absorptivity of solar radiation, and thus decrease the surface temperature of pavements/streets; (iv) in continuation of the previous point, natural and artificial cool materials (albedo values of 0.70 and 0.78 , respectively) were used in public spaces, and cool asphalt was applied on the roads; and lastly, (v) incorporation of water elements in most streets in order to promote the cooling effect through evaporation, and to directly cool local surface temperatures through the cooling effect of running water [30].

Case \#8 examines the use of cool pavements in an effort to address thermal comfort in a public space located within Tirana. Currently under construction, apart from the increase of green spaces, solar control pergolas, and earth to air heat exchangers, reflective pavements were a fundamental bioclimatic design feature. Beforehand, the public realm accommodated pavements that were extensively made up of dark concrete and/or stone tiles with an albedo range of between 0.15 and 0.20 [31]. The climatic analysis determined that the area suffered from both elevated ambient and surface temperatures during the summer period, and, as a result, thermal comfort levels were compromised.

The proposed design solutions included increasing vegetation, the use of shading, and the use of materials with appropriate thermal properties. This specifically included the: (i) extended use of shading and solar control in the area in order to reduce the surface temperature of materials and resulting heat convection; (ii) high trees to enhance shading and evapotranspiration in the considered area; (iii) use of light coloured materials to decrease the absorption of solar radiation and surface temperatures of local pavements (the albedo of the chosen materials all exceeded the value of 0.65 and presented significantly lower surface temperatures then those to be expected from conventional materials of the same colour); and lastly, (iv) limitations on the amount of local traffic in order to decrease the amount of local anthropogenic emissions [31].

The outcomes of the interventions were measured at a height of $1.50 \mathrm{~m}$ (in order to simulate pedestrian level) through the use of Computational Fluid Dynamics (CFD), which illustrate that ambient temperatures were considerably reduced, especially, in areas already prone to maximum temperatures as shown in Table 2.

Case \#9 is located in a highly populated area within the central zone of Athens, and it involves the use of cool pavements for streets and other open areas. Adjoining the changing of pavement materials, there is also an increase of greening/shading in the public spaces, and the use of earth to 
air heat exchangers. The original pavements were constituted of black asphalt on the roads, and the rest of the public realm was made of white tiles with an initial albedo of 0.45 (it should be noted, however, that the albedo decreased significantly as a result of wear and tear, especially in areas with heavier foot fall). After monitoring the local microclimate, it was concluded that the ambient and surface temperatures required alleviating, thus, presenting the opportunity for attenuation techniques. The overall bioclimatic approach included the use of: (i) concrete tiles which contained photocatalytic asphalt in the streets; (ii) concrete tiles coloured with infrared reflective paints; (iii) additional shading and green areas; and lastly, (iv) earth to air heat exchangers [32]. As shown in Table 2, such interventions suggest an overall decrease of up to $2 \mathrm{~K}$, an improved homogeneity in temperature ranges within the public space; and finally, a significant decrease in temperatures in both the eastern and western areas of the square, owing considerably to the incorporation of cool materials [32].

Finally, Case \#10 tackles the rehabilitation of an urban park in Athens that connects with the city's adjacent waterfront. Beforehand, most of the pavement in the park consisted of asphalt, concrete and dark paving, resulting in local albedo's ranging from 0.35 to 0.20 [23]. Through the monitoring of the existing microclimatic conditions during the summer, it was again concluded that a bioclimatic rehabilitation was required. In addition to the increase of vegetation and trees, cool pavements were the predominant measure used throughout the project. As a result, concrete pavements coloured with infrared reflective cool paints (presenting an albedo of 0.60) were installed in the park area. Through the use of visual infrared thermometer imaging, the difference in surface temperature between the shaded cool pavement, un-shaded cool pavement, and the surface temperature of a remaining, and moreover un-shaded, part of the previous pavement were examined. The temperature difference between the pavement specimens varied between $11.3 \mathrm{~K}$, thus suggesting the importance of not only the shade, but the thermal benefits of the cool materials as well.

When an overall analysis was undertaken to determine the site's spatial distribution of temperature, it was already expected that the area adjacent to the sea would benefit less from the presence of the new "cool" materials. This is due to the influence of maritime breezes, humidity, and overall proximity to the water. On the other hand, the cool pavements in the park's interior play a significant role in decreasing both surface and maximum ambient temperatures, as depicted in Table 2.

Similarly to Cases \#7-10, the following two cases integrate the use of materials as part of their bioclimatic approach in attenuating thermal comfort levels. Both cases also clearly aim at tackling UHI effects, and additionally, use it as part of their mission statement. The Parisian environmental redevelopment project (Case \#2) underpins: "A comfortable square, conscious of its environment-from an environmental point of view, traffic has been routed through the shaded area of the square to free up a large pedestrian area in the sunny part. (...) The process is underpinned by the use of perennial materials and economic techniques." ([19], p. 7). More specifically, local UHI was directly used as a design generator to reconfigure the area's surface materials, whereby: (i) the shady zones of the square were paved predominantly in darker colours; and, (ii) the open spaces were paved predominantly with generally paler colours. The "One Step 
Beyond Project" (Case \#3) also state in their proposal that "the benefit of using cool materials such as light asphalt, light concrete or light natural stones, is their high reflectivity and albedo. Cool materials guarantee less absorption of radiation and lower surface temperatures compared to other conventional materials. Through this reduction of heat storage in urban materials, the process of cooling down ambient air temperature at night accelerates...” ([33], p. 14).

\subsubsection{Scientific Incongruities between that of Surface Temperature and Ambient Temperature}

As the bearings of thermal discomfort continue to gain weight in local decision making and/or design (both resultant of current temperatures, and in congregation with those to be expected as a result of climate change), numerous incongruities regarding the use of materiality have been raised. More specifically, recent studies are now questioning the overall advantages of reflective materials that reduce the temperature of urban surfaces, such as pavements. To date, the benefits of reducing surface temperatures through high albedo roofs have palpably proven to reduce summertime building cooling energy requirements (Such evidence led to the United States Department of Energy launching the "Cool Roof Initiative" in 2010, and in an effort to urge others to their cause, Energy Secretary Steven Chu stated "Because cool roofs provide significant energy savings and environmental benefits, they should be used whenever practicable") ([34], p. 2). Contrariwise, and turning our attention back to the urban canyon, certain authors have recently suggested that the decrease of urban surfaces (such as that of pavements) may, in fact, not lead to a decrease in ambient temperature, and additionally even lead to "adverse human health impacts" [35]. Such authors also suggest that the reflection of radiation from high-albedo pavements can moreover: (i) increase the temperature of nearby walls and buildings; (ii) augment the cooling load of surrounding buildings; (iii) lead to heat discomfort felt by pedestrians; and lastly, (iv) induce harmful reflected UV radiation and surface glare [35].

Similarly to most fields within the spectrum of climatic adaptation, further investigation is required. Notwithstanding, this paper suggests that these incongruities should not hinder both the development and further incorporation of reflective materials, namely, that of cool pavements.

Firstly, and as recognized by authors such as [36,37], cool pavements can in fact lead to discomfort due to the increased budget of solar radiation (i.e., short-wave radiation) being approximately twice the decreased budget of long-wave radiation. As a result, this implies that relying only on the use of measures such cool materials can, counterproductively, lead to the thermal discomfort of pedestrians. Nevertheless, and as illustrated by Cases \#2,3,7-10, the use of reflective materials was part of a successful and wholesome bioclimatic intervention that aimed at decreasing ambient temperatures, and not just surface temperatures. In other words, the decrease of surface temperatures was combined with other passive strategies, such as, increasing shading and solar control, increasing vegetation, and limiting traffic.

Secondly, as shown in (Case \#9) researchers and manufacturers have also been developing cool coloured materials with higher reflectance values compared to conventionally pigmented materials of the same colour. These, encouragingly, have already been applied in cases where the use of light colours may lead to glare issues; or for simply when the aesthetics of darker colours are preferred [32]. 
Finally, and as classified by [27], cool materials for the built environment can be divided into two categories, cool materials for buildings, and cool paving materials. This paper suggests that the preoccupation with consequential thermal augmentation reflected from pavements onto nearby walls is, to some degree, controversial. Unlike pavements, the thermal attenuation of building surfaces has been well documented, and understood for almost half a century, hence architects/urban designers have recognised that reflective buildings' colours and/or materials can decrease building thermal loads. In juxtaposition, and when addressing outdoor thermal comfort, this infers that if a wall or building surface is affected by the pavement's increased reflectivity, the building surface is in itself thermally inefficient.

\subsubsection{Lessons for Auckland's CBD}

In the long term and when considering the implications of UHI effects in Auckland, it is essential to ruminate that the city is expected to grow by one million inhabitants by 2040 . Naturally, the increased urban density in juxtaposition with increased temperatures will lead to the effects of UHIs becoming an increasingly pressing issue for local thermal urban design. Similarly to most arenas associated with climatic adaptation, the necessity for further investigation and presence of both incongruities and uncertainties shall continually afflict local design makers and designers. Nevertheless, and considering the relevant lessons for Auckland's CBD, the cases presented in Table 2 have shown that materiality can play an effective, yet economical, way of tackling challenges such as UHI effects and the increase of annual hot days.

In addition, and considering that most of Auckland's CBD has an extensive amount of dark pavements, the deliberation upon surface albedo becomes a key issue in order to reduce ambient temperatures during the summer. Returning now to the example of Queen Street, both sides of the street are paved with dark stone slabs, while the road itself is composed of asphalt as shown in Figure 2a. As an exploratory exercise, Figure $2 \mathrm{~b}$ demonstrates how the street could look with the application of a cool pavement. In this fashion, the low reflectivity/emissivity rates of the previously dark pavement can be increased, where required, contingent on the extent of solar radiation exposure/hours.

In the case of Queen Street, Figure $2 b$ demonstrates how the thermal inertia can be varied in order to decrease the surface temperature of the pavements. By increasing the albedo within the major foot fall area (i.e., in front of retail frontages), the discharge of sensible heat and long-wave radiation can be reduced. When considering the energy balance of the pavement, the presence of shade (leading to a decrease in solar radiation/irradiance), and the increased albedo (leading to a decreased absorption and an increased reflection) the respective surface temperatures can be decreased. This decrease notwithstanding, it is also possible to consider the use of darker colours with a lower albedo under the shaded areas due to the decrease of solar radiation. This, however, would require careful consideration, as this could decrease the overall effectiveness of the pavement in lowering street temperatures. 


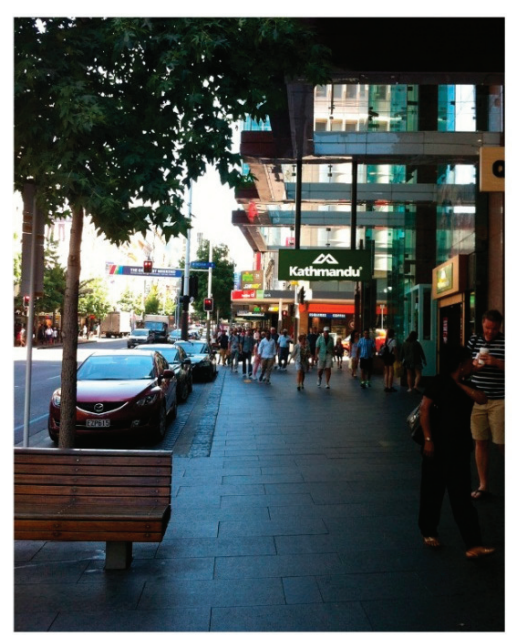

(a)

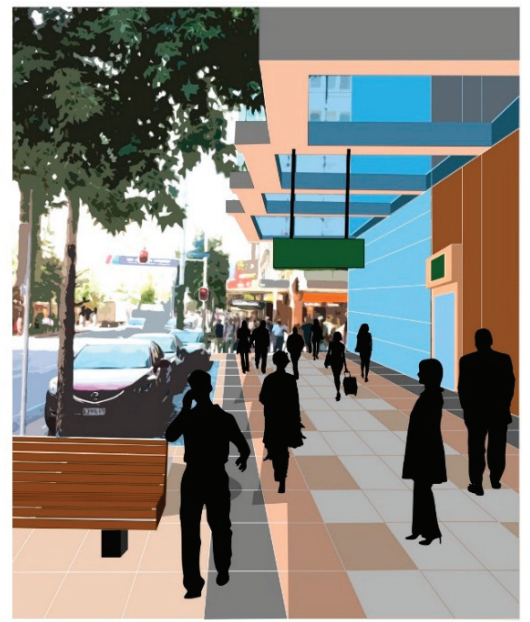

(b)

Figure 2. Conceptual intervention of cool pavements in Queen Street (a) Before the installation of cool pavements; (b) Post intervention. Source: Author's rendering.

\subsection{Water and Vapour Mechanisms}

This article has hitherto discussed the influences of vegetation, shelter canopies, and materiality upon thermal sensitive urban design. This section shall discuss the opportunities presented by water/vapour systems and shall examine their possible application in Auckland's public realm. In this section Relative Humidity $(\mathrm{RH})$ levels will be used in order to address the evaporative cooling potentials and processes of water. As this relates to the relationship between actual vapour pressure and saturated vapour pressure, this percentage enables the understanding of how water can be used to cool ambient temperature without exasperating ambient moisture levels. Previously, the presence of water and misting systems were customarily focused upon aesthetic and sculptural purposes in public space design. More recently, however, there has been a considerably greater emphasis upon their interconnection with bioclimatic comfort in outdoor spaces in terms of adaptation efforts to climatic conditions [38]. As a result, water and misting systems have taken on a new meaning in public space design.

\subsubsection{Lessons Learnt from the Direct Application of Evaporative Cooling Methods}

The first examples in this genre of strategy return to Cases \#2 and 3 that also incorporate the use of water and vapour systems as part of their bioclimatic intervention. In Case \#2, water is used both for aesthetic purposes and for attenuating elevated temperatures during the summer. During this season: (i) a fine sheet of water is released and incorporates the use of spraying systems upon a $1 \%$ slope over an area of $270 \mathrm{~m}^{2}$ (Figure 3a); and, (ii) the monument basin in the centre of the new pedestrian esplanade is filled with water through small water spouts (Figure 3b) [19]. As stipulated within the project brief, the utilization of water is primarily climatic, yet also designed to enhance 
the sociality, recreation and the aesthetics in the new Place de la Republique. It is worth reinforcing that this use of water in order to attenuate both temperatures, and UHI, is designed around a similar climate to that of Auckland, i.e., with a KG climate classification on "Cfb".

Case \#3 returns to the "One Step Beyond" project's "Heat mitigation Toolbox", that implements water measures that reduce the UHI effect and temperatures in its public spaces, such as Omonia square. More specifically, and serving as a focal point of the square, a fog-fountain is proposed to cool ambient temperatures through the evaporation of the water particles. Unlike Auckland, and due to its type of climate (i.e., "Csa"), Athens has very low RH levels, hence evaporative cooling is considerably more effective. As a result, and during a microclimatic analysis field study, the ambient air temperature peaked at $39^{\circ} \mathrm{C}$, while $\mathrm{RH}$ remained at only $30 \%$; with the aid of software projections, it was estimated that the evaporative system could aid lower surface temperatures down to $23{ }^{\circ} \mathrm{C}$ [22]. Counterproductively, the lower RH leads to another type of problem, the lack of water to sustain such systems. Yet with the use of other water elements, such as underground water storage systems, filtered rainwater can be used for both surface and greenery irrigation in periods of drought.
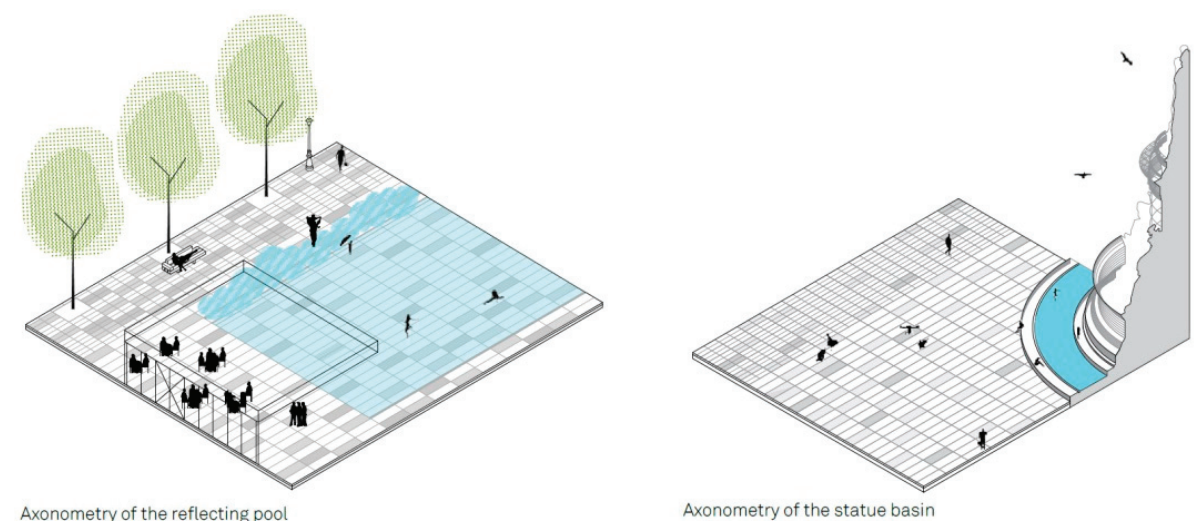

Figure 3. Axonometric of the (a) Reflecting pool; (b) Statue basin. Adapted with permission from Trévelo \& Viger-Kohler [19].

Following onto the next case, and awarded the first prize in a local competition to re-develop the Khan Antoun Bey Square in Beirut, PROAP Landscape Architects (Case \#11) explored a conceptual solution to improve outdoor thermal comfort standards. The dominant bioclimatic measure used in this project was a misting system, which in combination with vegetation, canopies and materiality, tackled hot-humid summers, and high solar radiation rates. This launched a deeper research into the effectiveness of temperature control systems in outdoor spaces by inducing evaporation through misting systems. The research concluded that misting-cooling systems can be complex, and its associated equilibrium with encircling air humidity is fundamental. In warm-humid summer climates, such as that of Auckland, water spraying and evaporation are more complex due to the existing amount of water already present in the atmosphere beneath the UCL. 


\subsubsection{The Equipoise between Evaporative Cooling and Relative Humidity Levels}

The effectiveness of evaporative techniques is one that is contingent on a variety of factors. Namely, and in order to efficiently lower ambient temperature without imperilling acceptable humidity levels, the correct water pressure, nozzle type, and functioning period must be established. Ultimately, and as proved by $[39,40]$, the formation of correct droplets with the adequate amount of temporal intervals becomes fundamental when addressing thermal comfort in areas with high humidity levels. In order to explore these techniques a little further, this paper shall discuss three case studies that adopt more of an engineering approach in order to establish actual temperature reductions through the means introduced in Table 3.

Historically, in Japanese culture, a rudimentary cooling method called "Uchimizu" was used to cool outdoor temperatures through the scattering of water upon the entrances of residential dwellings. In an effort to profit from previous teachings, Ishii, Tsujimoto, Yoon, and Okumiya [40] developed an exterior misting system that would reduce the air-conditioning load of encircling buildings without resorting to the use of vegetation (Case \#12). As Japan has a continental humid climate (i.e., with a KG classification of predominantly "Cfa"), the system was designed to overcome high humidity when attenuating high ambient temperatures, hence the name "Dry-Mist". In an outdoor environment, it was projected that: (i) for every $1 \mathrm{~K}$ drop in ambient temperature, $\mathrm{RH}$ would increase by 5\%; and; (ii) the system could lead to total decrease of $2{ }^{\circ} \mathrm{C}$ that, consequently, would lead to a reduction of $10 \%$ in energy consumption from air conditioners [40]. The atomization of the water particles resulting from the high pressure pump is connected to various meteorological sensors and control panels. Consequently, this enabled the automatic-control of the system, which would be triggered by certain pre-inserted environmental conditions. More specifically, the system would initiate when temperatures would surpass $28{ }^{\circ} \mathrm{C}$, when $\mathrm{RH}$ was below $70 \%$, and lastly, when the wind velocity was below that of $3 \mathrm{~m} / \mathrm{sec}$ without rainfall.

Although in a different setting, Case \#13 takes the exploration of the "Dry-Mist" a little further. Installed in a semi-open train station platform during the summer of 2007, a total of 30 "Dry-Mist" nozzles were installed to test their thermal cooling effect. This investigation, carried out by [39], demonstrated an initial cooling potential mean of $1.63 \mathrm{~K}$ and $1.9 \mathrm{~K}$ between 9:00 and 13:00 and 13:00 and 15:00, respectively. Yet, after obtaining such results, it was concluded that the operation period ( 2 min with an interval of $3 \mathrm{~min}$ ) was too short and that the mean cooling potential mean could thus be increased to $2 \mathrm{~K}$. Interestingly, and beyond these mean values, the maximum decrease in temperature reached $6 \mathrm{~K}$ and was accompanied by an increase of $28 \%$ of RH. It is worth noting that this consequential increase in humidity, although not in a fully exterior setting, accurately follows the ratio established in Case \#12 (i.e., $-1 \mathrm{k}=+5 \%$ of $\mathrm{RH}$ ). 


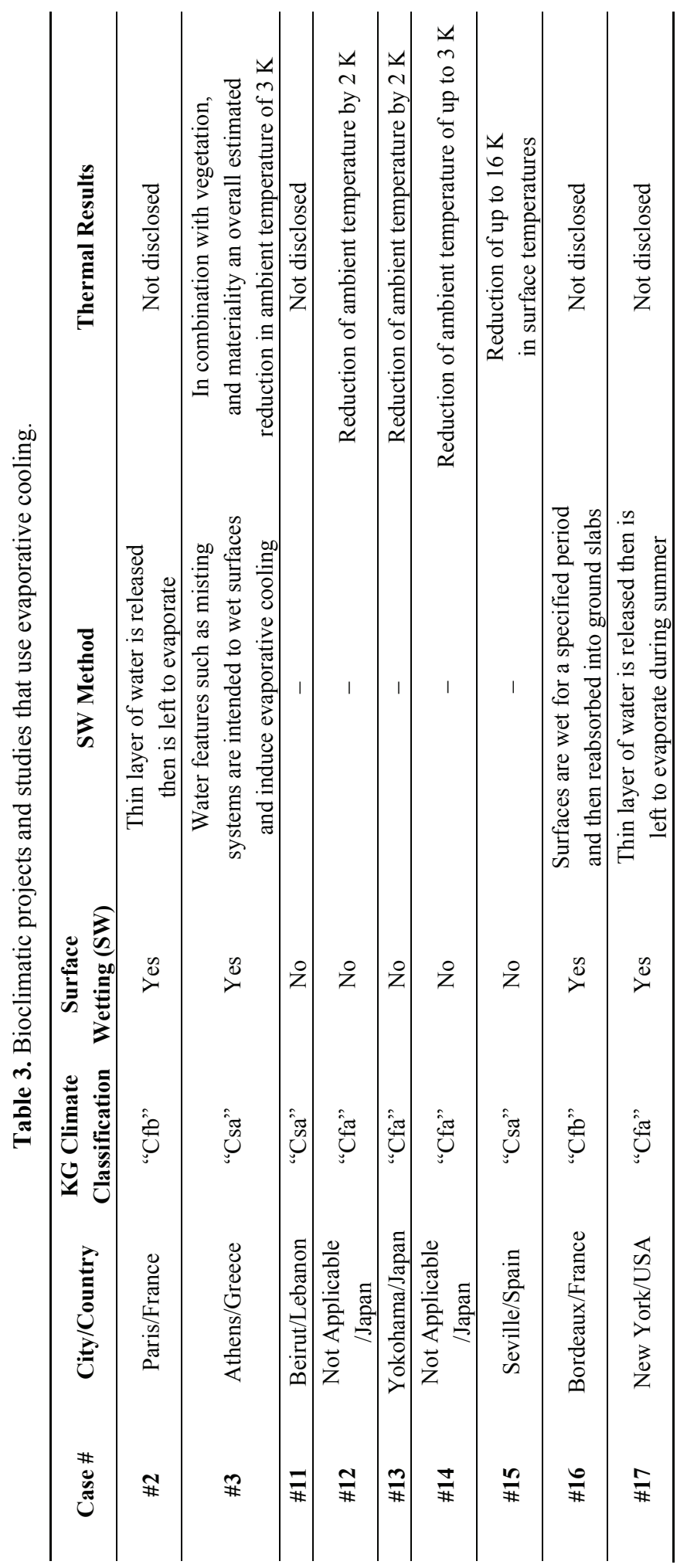


The analysis of the "Dry-Mist" was accompanied by various questionnaires in order to evaluate how the users of the platform reacted to the system. The results obtained from the questionnaires (Figure 4) demonstrated that: (i) $80 \%$ of the 200 respondents found the system at least "somewhat comfortable"; (ii) only $1 \%$ stated that they wanted the misting system to be stopped, and $98 \%$ stated that they enjoyed the presence of the system; and lastly; (iii) of this $98 \%, 21 \%$ asked for more mist, and another 57\% said that it was just right [39]. Hence, this case study has shown that even in conditions with high humidity, the careful and attuned use of misting systems can be both effective and successful.

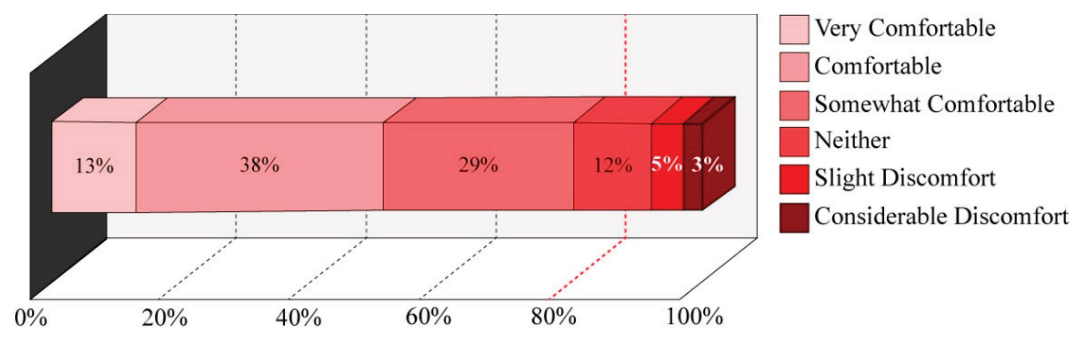

Figure 4. Results from thermal comfort questionnaire. Adapted with permission from CiNii [39]. 3.4.3. The Variation of the Sauter Mean Diameter and Spraying Height

As seen from the previous cases, misting systems can act efficiently in attenuating thermal comfort even in conditions with high humidity. Notwithstanding, and similar to the application of cool pavements discussed in the previous section, there is limited knowledge regarding actual design and installation. A quandary that often arises in this context is associated with the optimum amount and size of the particles, i.e., the Sauter Mean Diameter (SMD) of the water particles. In order to approach this issue, and for simplification purposes, Case \#14 joins three similar Japanese studies of misting systems together.

In the study undertaken by Yamada et al. [41], and through the use of CFD analysis, it was demonstrated that there is no significant difference in temperature reduction for different SMD sizes; however, it was identified that larger water particles $(\approx 32.6 \mu \mathrm{m})$ remain longer in the air. In design terms, this implies that since larger particles take longer to evaporate, spray height becomes a very important parameter. In the study of Yoon et al. [42], it was furthermore concluded that in any outside air temperature condition, when the RH goes beyond that of $80 \%$, and with the nozzles at a height of $0-0.25 \mathrm{~m}$, water particles would remain without evaporating. Consequently, ground surface wetting is to be expected, thus indicating that when Surface Wetting (SW) is undesired, the respective environment would not be suitable for spraying. Yet at a height of $1.5 \mathrm{~m}$, and given an $\mathrm{RH}$ of no more than $75 \%$, the misting system can efficiently cool ambient temperature without compromising overall thermal comfort [42]. Interestingly, and based on these results, it was considered that Japanese cities such as Tokyo, Osaka, and Fukuoka (all of which prone to elevated RH levels beneath the UCL) can considerably benefit from this evaporative cooling technique.

More recently, the last example in this case study was carried out by Farnham et al. [43], who verified both the importance of nozzle height, but also the fundamental role of SMD. Carried out in 
Osaka, and within a semi-enclosed space, this particular experiment achieved a total of cooling 0.7 $\mathrm{K}$ without SW. This was accomplished by single nozzles spraying mists with a SMD of $41-45 \mu \mathrm{m}$; moreover, the resultant increase of encircling humidity had little or no effect on the thermal comfort as demonstrated by the identified Effective Temperature (ET). These results notwithstanding, even from heights of $25 \mathrm{~m}$, if the SMDs were to be increased, an excessive amount of water particles would amalgamate close to the floor (hence over-increasing RH), and also cause undesired SW.

Cases \#12-14 have shown how Japan has overcome high humidity levels when cooling its public and semi-enclosed spaces. Inspired by an ancient and cultural practice, Japan is a front-runner country in the application of misting systems. Due to their technical approach, design orientated projects can learn from their methodical resolution to attenuating ambient temperatures and UHI through the use of misting systems.

Earlier, and within the European context, developed by an interdisciplinary group led by the department of Energy Engineering and Fluid Mechanics from the College of Industrial Engineering of Seville, the Expo of 1992 in Seville (Case \#15) was approached as a method to synthesis bioclimatic techniques with public space design. The various new techniques that were tested and installed concentrated on misting systems and bodies of water, namely the: (1) continuous blowing of air through a fan that was permanently kept moist; (2) installation of "micro" water nozzles in tree branches that created droplets with an average SMD of around $20.0 \mu \mathrm{m}$, where colder air then flowed downward, hence cooling the shaded areas; (3) "sheets" of water in the form of ponds and waterfalls that cooled the spaces through evaporative cooling and strategically placed irrigation outlets [44]. Integrated with vegetation, canopies, and materiality, the public realm of the Expo was divided into three different types of spaces: (1) "Passage Areas"-with the prime functionality of supporting the main flow of pedestrians, with an expected "use timeframe" of below 15 min; (2) "Rest/Stay Areas"-with the primary goal of offering places for resting, eating, and social congregation, with an expected "use timeframe" of over $15 \mathrm{~min}$; and lastly, (3) "Adjacent Areas"that were spaces of interconnectivity between the former. This theoretical division between Passage, Rest, and Adjacent areas aided thermal comfort design to be divided into medium level, high level, and low level thermal conditioning, respectively.

\subsubsection{SW within the Design Spectrum of Cooling Systems}

In the case of Seville, SW was undesired as it was argued that it would lead to stagnancy and resource wastage [44]. Although this is a valid argument, this does not imply that SW cannot be part of the system's overall design. In other words, the actual act of controlled surface wetting can be the method to cool down ambient temperatures and mitigate UHI effects. Respectively, Table 3 determines which cases allow SW, and additionally, how they assimilated this within the design of the cooling system.

A successful example of this tactic, and situated in a "Cfb" climate, the project Le Miroir d'Eau (Case \#16) by Michel Corajoud, Pierre Ganger, and Jean-Max Llorca, was initially aimed only at reintroducing vegetation into the space in order to attenuate the local microclimate. However, and based on the concept of addressing thermal comfort levels and reflecting surrounding facades, the "water mirror", and an incorporated on-site fog system (also based on a "micro" nozzle system) 
were installed. In order to avoid algae and water wastage, the water that temporarily floods the square recedes back into the slabs after a few minutes, leaving the surface dry like in any other square. Grooves were installed in-between the granite slabs, to allow the water to be recollected, and re-prepared for the next induced "flood". In this way, wet surfaces become part of the design of the system that increases the climatic responsiveness of the once thermally problematic public space.

Returning to the ephemeral perspective, and as already discussed through the "Canopy" project (Case \#6), misting systems and water bodies have also been translated into ETCSs within the public realm. In this scope, one can also refer to the "CoolStop" project (Case \#17) by Chat Travieso Design, which in collaboration with the NYC Department of Transportation, designed a temporary misting system during an annual event that pedestrianized seven miles of the city's streets. Constructed out of PVC piping, and operated through a hydrant unit, the misting system cooled the microclimate during the summer heat in New York's public spaces. Due to its ephemeral nature, resource wastage and stagnation is far less of a concern due to its on/off nature, seasonal use, and low water requirements.

\subsubsection{Lessons for Auckland's CBD}

Accordingly, and referring back to Table 1, Auckland's relatively high humidity levels need to be carefully deliberated when considering the application of water and misting mechanisms. As identified by Yoon, Yamada, and Okumiya [42], such mechanisms tend to be more intricate in attenuating thermal comfort levels when the RH surpasses the $75 \%$ mark. Nonetheless, this does not infer their inapplicability. Instead, three approaches can aid their applicability in Auckland's public realm, whereby: (1) SW is undesired-requiring careful consideration of necessary water pressure, nozzle type, altitude, and functioning period/intervals; (2) SW is desired and water is reused within the system - requiring hence water runoff deliberation; (3) ETCS are installed as a temporary measure during the summer period. Respectively, and referring to Table 3 , local designers and decision makers can refer to examples such as: (1) Cases \#11-15 to learn from existing studies and projects that contour high humidity levels and avert SW; (2) Cases \#2,3,16 to learn from precedents that incorporate SW into the design of the respective cooling mechanism; (3) Cases \#6 and 17 which present ephemeral evaporative solutions during the hotter months of the year.

These cases notwithstanding, it is still necessary in all approaches that local microclimatic factors are considered in order to fully exploit the potentiality/efficiency of such measures in attenuating thermal comfort levels through evaporative cooling.

\section{Framework Illustrations and Discussion}

As aforementioned, climate change adaptation has grown exponentially within both the global scientific and political arenas. Accordingly, one can witness the increasing global ambition amongst decision makers and designers to diminish the gap between theory and action with regards to local adaptation measures [45]. As shown in Figure 5, in order to introduce effective local 
climatic measures in Auckland's public realm without the risk of ineffectual adaptation (i.e., maladaptation), local agents must focalise their adaptation endeavours around specific local risk factors through a bottom-up attitude. In this way, existing knowledge within the adaptation agenda must subsequently be refined into an appropriate response through a "case by case" attitude.

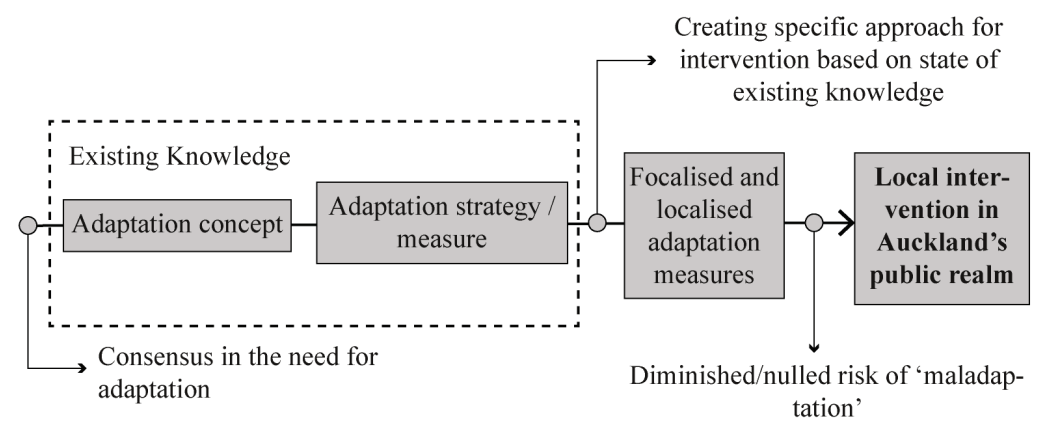

Figure 5. Proposing adaptation measures which focus upon Auckland's public realm and local risk factors. Adapted with permission from Taylor \& Francis [45].

All the same, the gap between theory and action with regards to thermal comfort attenuation is extensive, leading to a lack of precedential benchmarks, indicators, and examples that could otherwise aid local decision making and design. To address this issue, Table 4 divides the 17 bioclimatic case studies into built, conceptual, and scientific projects. In this way, the practical experience from actual construction, convergent thinking through conceptual exploration, and the empirical outcomes of scientific methodologies can be presented in the framework. Having Auckland as the central case study, the framework demonstrates know-how within the international arena with regards to similar microclimatic constraints that are already, or shall soon be, witnessed in the city.

\section{Methods of Incorporating the Framework}

Although some of the case studies were indeed based on warmer climates, they nevertheless suggest very pertinent benchmarks that can be adapted to New Zealand's more temperate climate. As discussed in the different sections of this article, these revisions can straightforwardly be undertaken by considering the microclimatic implications encircling Auckland's public realm. In this way, documents such as the regulatory UP, and non-regulatory Auckland's Design Manual (ADM), can introduce more concrete guidelines on how public spaces could be made more responsive in light of increased hot days, heat waves and managing UHI effects. More specifically, and now considering the ADM's "Section 4-Design for Comfort and Safety", the presented framework launches existing applicable bioclimatic solutions which can be made applicable for Auckland's CBD. Figure 6 demonstrates a possible online extension of Section 4 that is explicitly orientated towards "Dealing with Thermal Comfort \& Climate Change". 
Table 4. Framework of relevant bioclimatic case studies within the international arena.

\begin{tabular}{|c|c|c|c|c|c|c|}
\hline Case \# & Project Title & Genre/Status & Location & $\begin{array}{c}\text { Predominant } \\
\text { Measure Used * }\end{array}$ & $\begin{array}{l}\text { KG Climate } \\
\text { Classification }\end{array}$ & $\begin{array}{c}\text { Temporal } \\
\text { Scope }\end{array}$ \\
\hline$\# 1$ & Not Applicable & $\begin{array}{l}\text { Scientific } \\
(2012)\end{array}$ & $\begin{array}{l}\text { Athens/ } \\
\text { Greece }\end{array}$ & $V \& M$ & "Csa" & Long-Term \\
\hline$\# 2$ & $\begin{array}{c}\text { "Place de la } \\
\text { Republique" } \\
\text { Redevelopment }\end{array}$ & $\begin{array}{l}\text { Constructed } \\
\text { (2013) }\end{array}$ & $\begin{array}{l}\text { Athens/ } \\
\text { Greece }\end{array}$ & $V \&$ M \& W & "Csa" & Long-Term \\
\hline$\# 3$ & $\begin{array}{l}\text { "One Step } \\
\text { Beyond" }\end{array}$ & $\begin{array}{c}\text { Under } \\
\text { Construction } \\
(2013-2015)\end{array}$ & $\begin{array}{l}\text { Athens/ } \\
\text { Greece }\end{array}$ & $V \& S \& M \& W$ & "Csa" & Long-Term \\
\hline$\# 4$ & "Activity Tree" & $\begin{array}{c}\text { Conceptual } \\
(2013) \\
\end{array}$ & $\begin{array}{l}\text { Athens/ } \\
\text { Greece }\end{array}$ & $V \& S \& M$ & "Csa" & Long-Term \\
\hline$\# 5$ & $\begin{array}{l}\text { "This is not an } \\
\text { Umbrella" }\end{array}$ & $\begin{array}{c}\text { Conceptual } \\
(2008)\end{array}$ & $\begin{array}{l}\text { Madrid/ } \\
\text { Spain }\end{array}$ & $\mathrm{S}$ & "Csa" & ETCS \\
\hline$\# 6$ & "Canopy" & $\begin{array}{c}\text { Constructed } \\
(2004) \\
\end{array}$ & $\begin{array}{c}\text { New } \\
\text { York/USA }\end{array}$ & S \& W & "Cfa" & ETCS \\
\hline$\# 7$ & Not Applicable & $\begin{array}{c}\text { Under } \\
\text { Construction } \\
(2012) \\
\end{array}$ & $\begin{array}{l}\text { Athens/ } \\
\text { Greece }\end{array}$ & $V \& S \& M$ & "Csa" & Long-Term \\
\hline$\# 8$ & Not Applicable & $\begin{array}{l}\text { Scientific } \\
(2011)\end{array}$ & $\begin{array}{l}\text { Tirana/ } \\
\text { Albania }\end{array}$ & $V \& S \& M$ & "Csa" & Long-Term \\
\hline$\# 9$ & Not Applicable & $\begin{array}{l}\text { Scientific } \\
(2011)\end{array}$ & $\begin{array}{l}\text { Athens/ } \\
\text { Greece }\end{array}$ & $V \& S \& M$ & "Csa" & Long-Term \\
\hline$\# 10$ & Not Applicable & $\begin{array}{c}\text { Constructed } \\
(2012) \\
\end{array}$ & $\begin{array}{l}\text { Athens/ } \\
\text { Greece }\end{array}$ & $V \& M$ & "Csa" & Long-Term \\
\hline$\# 11$ & $\begin{array}{l}\text { "Khan Antoun } \\
\text { Bey Square" }\end{array}$ & $\begin{array}{c}\text { Scientific/ } \\
\text { Conceptual } \\
(2010) \\
\end{array}$ & $\begin{array}{c}\text { Beirut/ } \\
\text { Lebanon }\end{array}$ & $\mathrm{V} \& \mathrm{~W}$ & "Csa" & Long-Term \\
\hline$\# 12$ & Not Applicable & $\begin{array}{l}\text { Scientific } \\
(2009)\end{array}$ & -/Japan & W & "Cfa" & Long-Term \\
\hline$\# 13$ & Not Applicable & $\begin{array}{l}\text { Scientific } \\
(2008)\end{array}$ & $\begin{array}{c}\text { Yokohama/ } \\
\text { Japan }\end{array}$ & W & "Cfa" & ETCS \\
\hline$\# 14$ & Not Applicable & $\begin{array}{l}\text { Scientific } \\
(2008 / 11) \\
\end{array}$ & -/Japan & W & "Cfa" & Long-Term \\
\hline$\# 15$ & Expo'92 Seville & $\begin{array}{c}\text { Constructed } \\
(1992) \\
\end{array}$ & $\begin{array}{l}\text { Seville/ } \\
\text { Spain }\end{array}$ & $V \& S \& M \& W$ & "Csa" & Short-Term \\
\hline$\# 16$ & "Le Miroir d'Eau" & $\begin{array}{c}\text { Constructed } \\
\text { (2006) }\end{array}$ & $\begin{array}{l}\text { Bordeaux } \\
\text { /France }\end{array}$ & W & "Cfb" & Long-Term \\
\hline$\# 17$ & "CoolStop" & $\begin{array}{c}\text { Tested Prototype } \\
\text { (2013) }\end{array}$ & $\begin{array}{c}\text { New } \\
\text { York/USA }\end{array}$ & W & "Cfa" & ETCS \\
\hline
\end{tabular}

* V = Trees and Vegetation; $\mathrm{S}=$ Shelter Canopies; $\mathrm{M}=$ Materiality; $\mathrm{W}=$ Water and Vapour Mechanisms. 


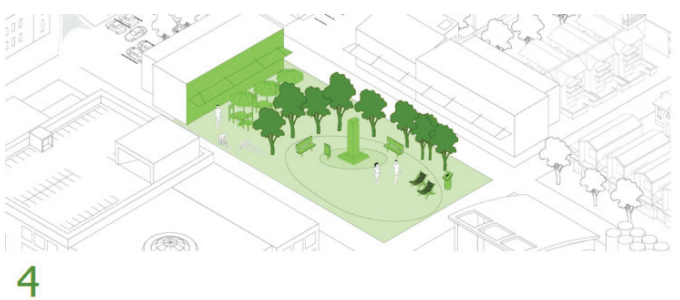

\section{Design For Comfort And Safety}

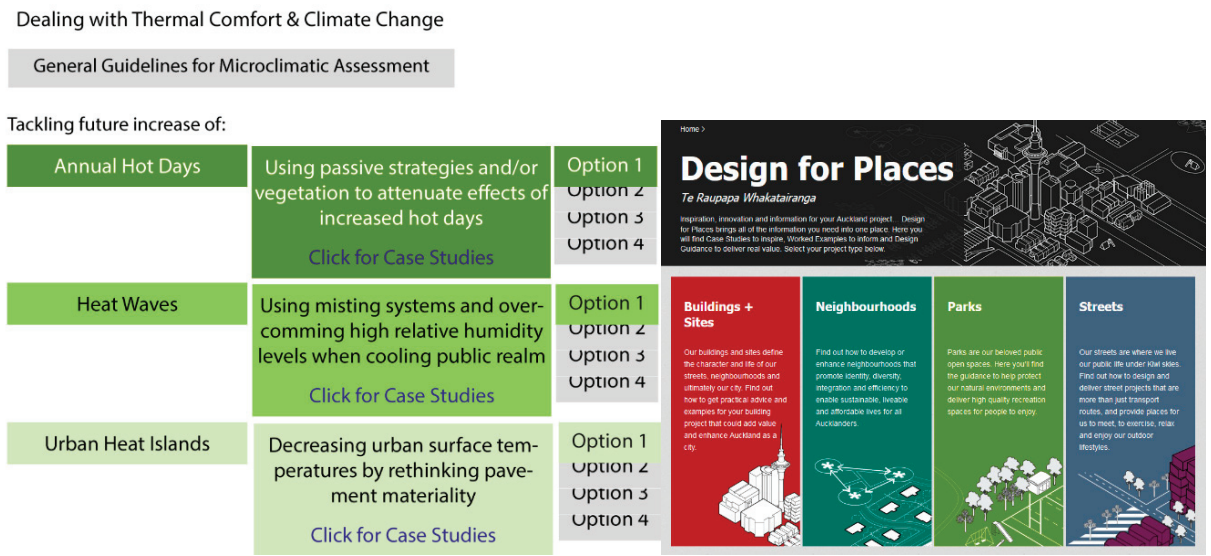

Figure 6. Extending the ADM through the incorporation of the framework.

Firstly, in order to provide both guidance on microclimatic assessment, and to avoid issues of maladaptation, a section on "General Guidelines for Microclimatic Assessment" was introduced. This aims at demonstrating simple and effective ways of examining local microclimate conditions, and to moreover explain the importance of such considerations both now and in the future.

Secondly, the tackling of increased hot days, heat waves and UHI effects can be met by different approaches and options which are discussed in the respective case studies. At the moment, the site contains references to the redevelopment of Aotea Square and Lumsden Green, yet this can be considerably extended in order to provide bioclimatic guidance on incorporating comfort and safety into the design and maintenance of Auckland's public spaces.

Respectively, this method shall advise means to, namely: (i) maximise the effects of local evapotranspiration in areas increasingly prone to UHI; (ii) effectively reduce/enable solar penetration and wind patterns; (iii) design suitable annual availability of choice between exposed, semi-shaded, and shaded areas (iv) support urban activity threads through passive strategies, evaporative cooling systems, and vegetation; (v) reduce surface temperatures through the implementation of cool materials; and, (vi) install misting systems that induce (or not) SW in order to attenuate local thermal comfort levels. In this light, the local design manual could hence form the basis for future action in Auckland's public spaces, which shall very likely require investigations into their: (i) use of passive strategies and/or vegetation to attenuate the effects caused by the 
increase of annual hot days; (ii) overcoming of difficulties presented by high RH levels when cooling the public realm; and, (iii) decreasing urban surface temperatures by rethinking the extensive use of dark pavements through the introduction of cool surfaces and materials (Figure 6).

\section{Conclusions}

As with most sectors in the maturing climate change adaptation agenda, there is considerable theory, yet limited practical benchmarks that can directly aid local decision making and design. Nevertheless, this article has argued that there is sufficient existing knowledge to respond to the growing need for thermal comfort attenuation in Auckland. Moreover, and although New Zealand shall witness more attenuated climate change over the next few decades, the discussed existing national projections nevertheless indicate that adaptation is still essential. On top of these meteorological projections, the considerable increase in population, urban density and $\mathrm{CO}_{2}$ emissions until 2040 augments such needs even further. To address such requirements, the presented framework of bioclimatic case studies has demonstrated a range of benchmarks that were developed by cities facing similar microclimatic issues to those that are present or expected in Auckland.

As a result, existing guidelines such as those pertaining to the city's public realm's comfort and safety can hence be developed further in order to aid local designers and decision makers to learn from existing approaches, and more importantly, to launch their own focalised approach. In this way, thermal sensitive urban design is launched into a fertile arena, whose application in a world of climate change is required in building a better New Zealand.

\section{Acknowledgments}

This paper would like to thank the AUSMIP Plus Program for providing the + Ph.D. Mobility Scholarship, which enabled the author's stay in New Zealand and the writing of this journal article. Secondly, to the Portuguese Foundation for Science and Technology for the individual doctorial grant (SFRH/BD/94521/2013). Additionally, a special thank you must be made to the "Transforming Cities: Innovations for Sustainable Futures" unit within the University of Auckland for their unremitting support. Lastly, the author would like to thank the Built Environment team within the Auckland council for their interest, and invitation to present the results disclosed in this article.

\section{Author Contributions}

The photographs and renderings illustrated in Figures 1 and 2 were taken and created by the author. Figure 5 contains adapted material that was created by the author during a previous conference presentation. The graphics of Figure 6 are based on the existing layout of the ADM's website, yet its content was altered for demonstrative purposes. 


$\begin{array}{ll}\text { Abbreviations } & \\ \text { GCMs } & \text { Global Circulation Models } \\ \text { KG } & \text { Köppen-Geiger } \\ \text { ENSO } & \text { El Nino-Southern Oscillation } \\ \text { IPO } & \text { Interdecadal Pacific Oscillation } \\ \text { NIWA } & \text { National Institute of Water and Atmosphere } \\ \text { UV } & \text { Ultra Violet } \\ \text { UP } & \text { Unitary Plan } \\ \text { PET } & \text { Physiologically Equivalent Temperature } \\ \text { UHI } & \text { Urban Heat Island } \\ \text { ARPHS } & \text { Auckland Regional Public Health Service } \\ \text { RH } & \text { Relative Humidity } \\ \text { SMD } & \text { Sauter Mean Diameter } \\ \text { SW } & \text { Surface Wetting } \\ \text { UCL } & \text { Urban Canyon Layer } \\ \text { CFD } & \text { Computational Fluid Dynamics } \\ \text { PMV } & \text { Predicted Mean Vote } \\ \text { ET } & \text { Effective Temperature } \\ \text { ADM } & \text { Auckland's Design Manual }\end{array}$

\section{Conflicts of Interest}

The author declares no conflict of interest.

\section{References}

1. Auckland Council. The Proposed Auckland Unitary Plan (Notified 30 September 2013); Auckland Council: Auckland, New Zealand, 2013.

2. Peel, M.; Finlayson, B.; McMahon, T. Updated world map of the koppen-geiger climate classification. J. Hydrol. Earth Syst. Sci. 2007, 11, 1633-1644.

3. Climate Change Effects and Impacts Assessment: A Guidance Manual for Local Government in New Zealand; Ministry for the Environment: Wellington, New Zealand, 2008; p. 149.

4. Semadeni-Davies, A. National-Climate-Centre: State of the Climate 2013; National Institute of Water \& Atmospheric Research (NIWA): Wellington, New Zealand, 2013; p. 109.

5. Mackintosh, L. Mean Relative Humidity for Selected Locations through New Zealand, by Month-1981-2010 (Dataset); National Institute of Water and Atmospheric Research (NIWA): Auckland, New Zealand, 2013.

6. Mackintosh, L. Climate Summaries-Annual Rain, Temperature, Sunshine, Frost, and Wind Data for Selected Locations through New Zealand-1981-2010 (Dataset); National Institute of Water and Atmospheric Research (NIWA): Auckland, New Zealand, 2013.

7. Gluckman, P. New Zealand's Changing Climate and Oceans: The Impact of Human Activity and Implication for the Future; Office of the Prime Minister's Science Advisory Committee (PMCSA): Auckland, New Zealand, 2013; p. 22. 
8. Oliveira, S.; Andrade, H. An initial assessment of the bioclimatic comfort in an outdoor public space in lisbon. Int. J. Biometeorol. 2007, 52, 69-84.

9. Katzschner, L. Microclimatic Thermal Comfort Analysis in Cities for Urban Planning and Open Space Design; Network for Comfort and Energy Use in Buildings (NCUB): London, UK, 2006.

10. Nouri, A.S. A Bottom-up Perspective upon Climate Change-Approaches towards the Local Scale and Microclimatic Assessment. In Green Design, Materials and Manufacturing Processes; Bártolo, H., Ed.; Taylor \& Francis: Lisbon, Portugal, 2013; pp. 119-124.

11. Olgyay, V. Design with Climate, Bioclimatic Approach to Architectural Regionalism; Princeton University Press: Princeton, NJ, USA, 1963.

12. Costa, J.P. "Climate Proof Cities". Urbanismo e a Adaptação às Alterações Climáticas. As Frentes de Água; Universidade Técnica de Lisboa: Lisboa, Portugal, 2011; p. 218. (In Portuguese)

13. Nikolopoulou, M. Designing Open Spaces in the Urban Environment: A Biocliamtic Approach. Available online: http://www.cres.gr/kape/education/1.design_guidelines_en.pdf (accessed on 4 March 2015).

14. Erell, E.; Pearlmutter, D.; Williamson, T. Urban Microclimate-Designing the Spaces between Buildings; Earthscan: London, UK, 2011.

15. Almeida, A.L.B. O valor das Árvores: Árvores e Floresta Urbana de Lisboa; Instituto Superior de Agronomia: Lisbon, Portugal, 2006. (In Portuguese)

16. Tsiros, I. Assessment and energy implications of street air temperature cooling by shade trees in Athens (Greece) under extremely hot weather conditions. J. Renew. Energy 2010, 35, 1866-1869.

17. Picot, X. Thermal comfort in urban spaces: Impact of vegetation growth. Case study: Piazza della scienza, milan italy. Energy Build. 2004, 36, 329-334.

18. Shashua-Bar, L.; Tsiros, I.X.; Hoffman, M. Passive cooling design options to ameliorate thermal comfort in urban streets of a mediterranean climate (Athens) under hot summer conditions. Build. Environ. 2012, 57, 110-119.

19. Trevelo \& Viger-Kohler Architectes Urbanistes (TVK). Place de la Republique; TVK: Paris, France, 2013; p. 7.

20. Auckland Regional Public Health Service (ARPHS). Feedback to the Draft; ARPHS: Auckland, New Zealand, 2013; p. 78.

21. Whyte, W.H. The Social Life of Small Urban Spaces; Project for Public Spaces Inc.: New York, NY, USA, 1980.

22. Knuijt, M. One Step Beyond. Open Space 2013. Available online: http://www.okra.nl/wpcontent/uploads/2014/07/2014_OKRA_Topos-85_-Knuijt.pdf (accessed on 6 March 2015).

23. Santamouris, M.; Gaitani, N.; Spanou, A.; Salirai, M.; Giannopoulou, K.; Vasilakopoulou, K.; Kardomateas, T. Using cool paving materials to improve microclimate of urban areas-Design realization and results of the flisvos project. J. Build. Environ. 2012, 53, 128-136.

24. Gustavsson, T.; Bogren, J.; Green, C. Road climate in cities: A study of the stockholm area, south-east sweden. J. Meteorol. Appl. 2001, 8, 481-489. 
25. Santamouris, M. Using cool pavements as a mitigation strategy to fight urban heat islands-A review of the actual developments. J. Renew. Sustain. Energy Rev. 2013, 26, 224-240.

26. Doulos, L.; Santamouris, M.; Livada, I. Passive cooling of outdoor urban spaces. The role of materials. J. Sol. Energy 2004, 77, 231-249.

27. Synnefa, A.; Santamouris, M.; Livada, I. A study of the thermal performance of reflective coatings for the urban environment. J. Sol. Energy 2006, 80, 968-981.

28. Georgakis, C.; Santamouris, M. Experimental investigation of air flow and temperature distribution in deep urban canyons for natural ventilation purposes. J. Energy Build. 2006, 38, 367-376.

29. Stathopoulou, M.; Synnefa, A.; Cartalis, C.; Santamouris, M.; Karlessi, T.; Akbari, H. A surface heat island study of athens using high-resolution satellite imagery and measurements of the optical and thermal properties of commonly used building and paving materials. J. Sustain. Energy 2009, 28, 59-76.

30. Santamouris, M.; Xirafi, F.; Gaitani, N.; Saliari, M.; Vassilakopoulou, K. Improving the microclimate in a dense urban area using experimental and theoretical techniques-The case of marousi, athens. Int. J. Vent. 2012, 11, 1-16.

31. Fintikakis, N.; Gaitani, N.; Santamouris, M.; Assimakopoulos, M.; Assimakopoulos, D.; Fintikaki, M.; Albanis, G.; Papadimitriou, K.; Chryssochoides, E.; Katopodi, K.; et al. Bioclimatic design of open public spaces in the historic centre of tirana, albania. Sustain. Cities Soc. 2011, 1, 54-62.

32. Gaitani, N.; Spanou, A.; Saliari, M.; Vassilakopoulou, K.; Papadopoulou, K.; Pavlou, K.; Santamouris, M.; Lagoudaki, A. Improving the microclimate in urban areas: A case study in the centre of Athens. Build. Serv. Eng. Res. Technol. 2011, 32, 53-71.

33. Presentation-European Architectural Competition for the Creation of a New City Centre; OKRA Landscape Architects: Delft, The Netherlands, 2013; p. 14.

34. Chu, S. Memorandum for Heads of Department Elements: Installation of Cool Roofs on Department of Energy Buildings; Department of Energy of the United States of America: Washington, DC, USA, 2010; p. 2.

35. Yang, J.; Wang, Z.; Kaloush, K. Unintended Consequences-A Research Synthesis Examining the Use of Reflective Pavements to Mitigate the Urban Heat Island Effect (Revised April 2014); Arizona State University National Center for Excellence for SMART Innovations: Tempe, AZ, USA, 2013.

36. Nakaoukubo, K.; Hoyano, A. Numerical analysis on radiant environment of outdoor living space considering the influence of spatial form and material. J. Environ. Eng. 2006, 73, 957-964.

37. Kondo, Y.; Ogasawara, T.; Udoh, K. Influence of radiation by cool pavement on thermal sensation of pedestrians. J. Environ. Eng. 2006, 74, 323-330.

38. Nunes, J.; Zolio, I.; Jacinto, N.; Nunes, A.; Campos, T.; Pacheco, M.; Fonseca, D. Misting-Cooling Systems for Microclimatic Control in Public Space; PROAP Landscape Architects: Lisbon, Portugal, 2013; pp. 1-16. 
39. Ishii, T.; Tsujimoto, M.; Yamanishi, A. The Experiment at the Platform of Dry-Mist Atomization. In Proceedings of the Annual Meeting of the Architectural Institute of Japan, Tokyo, Japan, 20 July 2008.

40. Ishii, T.; Tsujimoto, M.; Yoon, G.; Okumiya, M. Cooling System with Water Mist Sprayers for Mitigation of Heat-Island. In Proceedings of the Seventh International Conference on Urban Climate, Yokohama, Japan, 29 June 2009.

41. Yamada, H.; Yoon, G.; Okumiya, M.; Okuyama, H. Study of cooling system with water mist sprayers: Fundemental examination of particle size distribution and cooling effects. J. Build. Simul. 2008, 1, 214-222.

42. Yoon, G.; Yamada, H.; Okumiya, M. Study on a Cooling System Using Water Mist Sprayers, System Control Considering Outdoor Environment. In Proceedings of the Korea-Japan Joint Symposium on Human-Environment Systems, Cheju, Korea, 29-30 November 2008.

43. Farnham, C.; Nakao, M.; Nishioka, M.; Nabeshima, M.; Mizuno, T. Study of mist-cooling for semi-enclosed spaces in Osaka, Japan. J. Urban Environ. Pollut. 2011, 4, 228-238.

44. Velazquez, R.; Alvarez, S.; Guerra, J. Control Climatico de los Espacios Abiertos En Expo 1992; College of Industrial Engineering of Seville: Seville, Spain, 1992. (In Spanish)

45. Nouri, A.S.; Matos Silva, M. Climate Change Adaptation and Strategies: An Overview. In Green Design, Materials and Manufacturing Processes; Bártolo, H., Ed.; Taylor and Francis: Lisbon, Portugal, 2013; pp. 501-507. 
MDPI AG

Klybeckstrasse 64

4057 Basel, Switzerland

Tel. +4161683 7734

Fax +41613028918

http://www.mdpi.com/

Buildings Editorial Office

E-mail: buildings@mdpi.com

http://www.mdpi.com/journal/buildings 


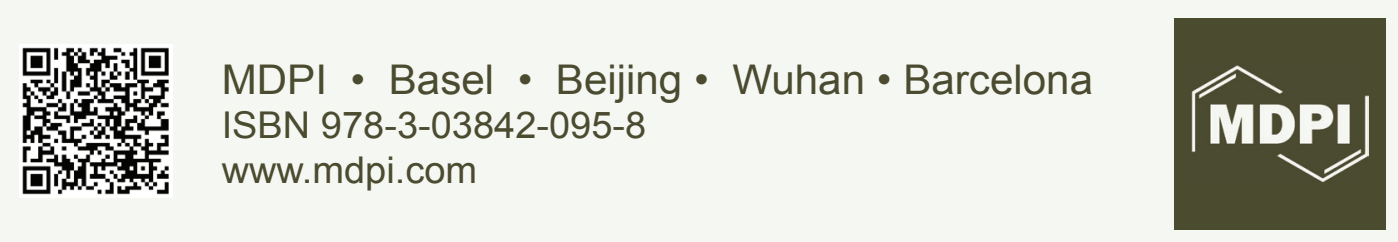